C. 2

\title{
Synopsis of the
}

\begin{tabular}{l} 
Parasites of \\
Vertebrates \\
\hline of Canada
\end{tabular}

\section{A Guide to}

\section{the Animal Parasites of Albertan Fishes}



A GUIDE TO THE

ANIMAL PARASITES OF ALBERTAN FISHES 


\section{Published by:}

Alberta Agriculture, Food and Rural Development Information Packaging Centre

7000 - 113 Street

Edmonton, Alberta T6H 5T6

Production Editor: Chris Kaulbars

Cover Design: John Gillmore

Copyright $\odot 2000$. Her Majesty the Queen in Right of Alberta. All rights reserved.

No part of this publication may be reproduced, stored in a retrieval system, or transmitted in any form or by any means, electronic, mechanical photocopying, recording, or otherwise without written permission from the Information Packaging Centre, Alberta Agriculture, Food and Rural Development. 
Other titles in this series available from the

Queen's Printer, 11510 Kingsway Avenue, Edmonton, Alberta T5G 2Y2

Synopsis of the Parasites of Vertebrates of Canada

--Helminths and Protozoa of Terrestrial Mammals

Murray J. Kennedy, 1986,90 p. $\$ 5.00$

Synopsis of the Parasites of Vertebrates of Canada

--Ectoparasites of Terrestrial Mammals

Murray J. Kennedy and Robert A. Newman, 1986,109 p. $\$ 5.00$

Synopsis of the Digenea of Mammals of North America

Murray J. Kennedy, 1988,83 p. $\$ 5.00$

Synopsis of the Parasites of Vertebrates of Canada

-- Parasites of Marine Mammals

Leo Margolis and Hisao P. Arai, 1989, 26 p. $\$ 5.00$

Synopsis of the Parasites of Vertebrates of Canada

-- Ectoparasites of Birds

Terry A. Wheeler and William Threlfall, 1989. 93 p. $\$ 5.00$

Synopsis of the Parasites of Vertebrates of Canada

-- Haemoprotozoans of Birds

G.F. Bennett, C.B. Woodworth-Lynas and M. A. Bishop, 1989, 47 p. $\$ 5.00$

Synopsis of the Parasites of Vertebrates of Canada

-- Cestodes of Waterfowl

J. Daniel McLaughlin, 1989, 21 p. $\$ 5.00$

Synopsis of the Parasites of Vertebrates of Canada

-- Nematodes of Birds

Pooi Leng Wong, Cheryl M. Bartlett, Lena N. Measures, Marilyn A. McNeill, R.C. Anderson, 1990,44 p. $\$ 5.00$

Synopsis of the Parasites of Vertebrates of Canada

-- A partially annotated bibliography of meningeal worm Parelaphostrongylus tenuis (Nematoda) and its close relatives

W.M. Samuel, 1991,36 p. $\$ 5.00$

\section{Publications available from Alberta Agriculture, Food and Rural Development, Publications Office, 7000 - 113 Street, Edmonton, Alberta T6H 5T6}

Synopsis of the Parasites of Domesticated Mammals of Canada

Murray J. Kennedy and David T. Moraiko, $1998,3^{\text {rd }}$ edition, 89 p. $\$ 10.00$

Veterinary Parasitology: Laboratory Procedures

Murray J. Kennedy, 1998, 85 p. $\$ 30.00$, Agdex \#650-1 
Digitized by the Internet Archive in 2016 


\section{INTRODUCTION}

From the outset, it should be noted that the title of this compendium is a misnomer. Initially, the thoughts were simply to compile records, originating in Alberta, of the animal parasites of fishes. However, it became clear that such a compilation would tend to give an impression of a depauperate fauna, when in fact, large numbers of parasites do and could potentially infect these fish species. Therefore, it was decided that all Canadian reports of parasites associated with Albertan fish species would be included. It is obvious that with the advent of water diversions, hydroelectric develcpments and fish transfaunations through artificial stocking of water bodies that information on potential parasitism is necessary and this information is made available with this extended treatment.

This guide is composed of two main sections: one giving Parasite/Host listings and the other giving Host/Parasite listings; also included are indices for the parasites and for the hosts, followed by a list of references.

The format adopted for the Parasite/Host listings include: 1) the currently accepted scientific name of the parasite, including the author and date; 2) synonyms, if included, are referrable only to those used in the Canadian context; 3) sites where the parasite might be found, in or on the host(s); 4) an alphabetical listing of all hosts, with the numbers following each, representing the numbered references which are cited as records; 5) the geographical distribution of the parasite is indicated by the use of abbreviations adopted by Canada Post for provinces and territories: $A B=A l b e r t a$, $\mathrm{BC}=$ British Columbia, $\mathrm{MB}=$ Manitoba, $\mathrm{NB}$ $=$ New Brunswick, NF $=$ Newfoundland, NS = Nova Scotia, NT = Northwest Territories, ON
$=$ Ontario, $\mathrm{PE}=$ Prince Edward Island, $\mathrm{QC}=$ Quebec, SK = Saskatchewan, and YT = Yukon Territory. Additional abbreviation used are: $\mathbf{A R}=$ Arctic, $\mathrm{CA}=$ Canada, $\mathrm{CCA}=$ central Canada, EXP = experimentally induced infections, $\mathrm{HBD}=$ Hudson Bay drainage (within Manitoba and Saskatchewan), LB = Labrador (as distinct from insular Newfoundland), SSR = South Saskatchewan River, WCA = western Canada and 6) records which are specific citations on the parasites. In instances where an Albertan record is indicated, the citation is in "bold print" and the locality in the province (if specified) is included.

The Host/Parasite list is organized according to the phylogenetic relationships of the host taxa. At the generic level, these taxa are listed alphabetically. The scientific and common names of the fish species follow Robins et al (1991) and the names of the higher categories and their phylogenetic arrangment follow Nelson (1984). Nelson and Paetz (1992) list 59 species of fishes in the Albertan fauna. Three of these species are obviously exotics, i.e. two poeciilids and a cichlid. Of the remaining 56, no parasites have been reported from Canadian populations of Hybognathus argyritis, Notropis blennius, Oncorhynchus aguabonita, nor from those of Cottus confusus. The splake (Salvelinus fontinalis $\mathbf{x}$ Salvelimus namaycush) is not listed by Nelson and Paetz (loc. cit.) as part of the Albertan fauna. However, the hybrid is included in this work since a larval tapeworm, Diphyllobothrium sp. has been recorded from this host in Alberta (Mudry and Anderson 1977); with this inclusion, 53 taxa of host species are considered. The listing of the major groups of parasites follows a 
phylogenetic sequence with the parasitic taxa in each group listed alphabetically.

In this guide, 329 specifically identified taxa of parasites and 104 others (with varying degrees of precision in identification) are considered. It should be noted that, upon further examination, many included in the latter group could be assigned to specifically named taxa, thereby reducing the numbers of what appears to be a very large parasite fauna. The total of 433 represents the information obtained and collated from Canada-wide reports of parasites from the $59-60$ species which constitute the fish fauna of Alberta.

The group-by-group (Canada vs. Alberta) totals of the parasites can be seen from the following: Cnidaria ( $1-1 ; 0-0)$, Monogenea (81-13; 7-4), Digenea (79-26; 15-5), Cestoidea (51-20;20-12), Nematoda (42-22; 12-6), Acanthocephala (22-6; 9-2), Annelida (16-5; 4-0), Mollusca (7-3; 1-1), Branchiura (16-5; 1-0), Copepoda (25-5; 5-3), and Arachnoidea (0-2; 0-0). In this listing (using the Monogenea as an example), the first set of figures (i.e. 81-13) indicates the Canada-wide reports with the " 81 " representing the spccifically identified taxa and the " 13 " representing those with other levels of identification. The second set (i.e. 7-4) represents the equivalent values for reports originating in Alberta.

Throughout this work, larval stages of the parasite are designated with an asterisk (*) preceding the name; a name preceded by two asterisks $\left({ }^{* *}\right)$ indicates that both larval and adult stages are included; a name without any special designation is that of the adult form of the parasite. 
PARASITE-HOST LIST 
KINGDOM ANIMALIA

SUBKINGDOM EUMETAZOA

PHYLUM CNIDARIA

CLASS HYDROZOA

ORDER TRACHYLINA

SUBORDER NARCOMEDUSAE

FAMILY CUNINIDAE

Polypodium hydriforme Ussov, 1885

Site: ova

Host: Acipenser fulvescens

Distribution: MB, SK

Records: Choudhury \& Dick 1991; Dick et al. 1991; Choudhury \& Dick 1993

Polypodium sp.

Site: ova

Host: Acipenser fulvescens

Distribution: MB, SK

Record: Dick et al. 1991

PHYLUM PLATYHELMINTHES

CLASS MONOGENEA
SUBCLASS POLYONCHOINA

ORDER DACTYLOGYRIDEA

SUBORDER DACTYLOGYRINEA

FAMILY DACTYLOGYRIDAE

SUBFAMILY ANCYROCEPHALINAE

Aethycteron malleus (Mueller, 1938)

Suriano and Beverley-Burton, 1982

Synonyms: Urocleidus malleus

(Mueller,1938) Mizelle and Hughes, 1938; Cleidodiscus malleus Mueller, 1938

Site: gills

Host: Percina caprodes

Distribution: ON

Records: Dechtiar 1972a; Dechtiar 1972b; Dechtiar \& Christie 1988; Dechtiar et al. 1988

Aethycteron sp.

Site: gills

Host: Etheostoma exile

Distribution: ON

Record: Dechtiar \& Christie 1988

Cleidodiscus brachus Mueller, 1938

Synonym: Urocleidus brachus (Mueller, 1938) Price, 1967

Site: gills 
Hosts: Couesius plumbeus (3)

Margariscus margarita $(4,5,6)$

Phoxinus eos (4)

Phoxinus neogaeus (1)

Platygobio gracilis (2)

Distribution: $\quad$ AB, LB, NB, ON

Records: 1) Dechtiar 1972a (ON);

2) Arai \& Chien 1973 (AB - Milk

River, Oldman River, St. Mary River);

3) Threlfall 1974 (LB); 4) Cone 1980

(NB); 5) Dechtiar et al. 1988 (ON);

6) Dechtiar et al. 1989 (ON)

Cleidodiscus sp.

Site: gills

Hosts: Perca flavescens $(1,2)$

Percopsis omiscomaycus (2)

Stizostedion vitreum $(3,4)$

Distribution: ON

Records: 1) Mizelle \& Donahue 1944;

2) Dechtiar 1972b; 3) Anthony 1976;

4) Anthony $1978 b$

Ligictaluridus posthon Klassen, BeverleyBurton and Dechtiar, 1985

Site: gills

Host: Noturus flavus

Distribution: ON

Record: Klassen et al. 1985

Ligictaluridus pricei (Mueller, 1936)

Beverley-Burton, 1984

Synonym: Cleidodiscus pricei Mueller, 1936

Site: gills

Host: Noturus flavus

Distribution: ON
Records: Dechtiar 1972b; Dechtiar \& Christie 1988; Dechtiar et al. 1988

Onchocleidus chautauquensis (Mueller, 1938) Murith and Beverley-Burton, 1984

Synonym: Urocleidus chautauquensis (Mueller, 1938) Mizelle and Hughes, 1938

Site: gills

Host: Perca flavescens

Distribution: ON

Record: $\quad$ Molnar et al. 1974

Urocleidus aculeatus (Van Cleave and

Mueller, 1932) Mueller, 1934

Synonym: Cleidodiscus aculeatus (Van

Cleave and Mueller, 1932) Mizelle and

Regensberg, 1945

Site: gills

Hosts: Perca flavescens $(4,5)$

Stizostedion canadense $(1,2)$

Stizostedion vitreum $(1,2,3,4,6$,

$7,8,9,10)$

Distribution: $\mathrm{MB}, \mathrm{ON}$

Records: 1) Dechtiar 1972a (ON);

2) Dechtiar 1972b (ON); 3) Anthony 1976 (ON); 4) Anthony 1978b (ON); 5)

Suriano \& Beverley-Burton 1981 (ON);

6) Poole \& Dick 1985 (MB); 7) Dechtiar

\& Christie 1988 (ON); 8) Dechtiar et

al.1988 (ON); 9) Dechtiar \& Lawrie 1988

(ON); 10) Dechtiar \& Nepszy 1988 (ON)

Urocleidus adspectus Mueller, 1936

Synonym: Cleidodiscus adspectus

Mueller, 1936

Includes: Cleidodiscus sp. of Mizelle and Donahue (1944) 
Site: gills

Hosts: Etheostoma exile (10)

Perca flavescens (1, 2, 3, 4, 5, 6, $7,8,9,10,11,12,13,14,15,16,17,18$, $19,20,21,22,23,24,25,26,27,28,29$, 30)

Percopsis omiscomaycus (28) Stizostedion vitreum (28)

Distribution: $\mathbf{A B}, \mathrm{MB}, \mathrm{NB}, \mathrm{ON}$

Records: 1) Mizelle \& Donahue 1944

(ON); 2) Tedla \& Fernando 1969a (ON);

3) Tedla 1969 (ON); 4) Tedla \& Fernando 1970a (ON); 5) Hanek 1971 (ON); 6)

Tedla \& Fernando 1972 (ON); 7) Hanek \& Fernando 1972a (ON); 8) Hanek \& Fernando 1972b (ON); 9) Dechtiar 1972a (ON); 10) Dechtiar 1972b (ON); 11) Kakonge 1972 (ON); 12) Molnar et al. 1974 (ON); 13) Cone 1978 (NB); 14) Cone 1979a (NB); 15) Cone 1979b (NB); 16) Cone 1979c (NB); 17) Cone 1980 (NB); 18) Cone \& Burt 1981 (NB); 19) Cone \& Burt 1982a (NB); 20) Cone \& Burt 1982b (NB); 21) Cone \& Burt 1985 (NB); 22) Poole \& Dick 1985 (MB); 23)

Dechtiar \& Christie 1988 (ON); 24)

Dechtiar et al. 1988 (ON); 25) Dechtiar \& Lawrie 1988 (ON); 26) Dechtiar \& Nepszy1988 (ON); 27) Szalai \& Dick 1991a (MB); 28) Szalai et al. 1992 (MB); 29) Zelmer 1994 (AB - Burnstick Lake, Carburn Pond, Coal Lake, Elkwater Lake, Fincastle Lake, Garner Lake, Pigeon Lake, Pine Lake, Rattlesnake Lake, Sherburne Lake, Sylvan Lake; 30) Zelmer \& Arai, 1998 (AB- Garner Lake)

Urocleidus baldwini (Dechtiar, 1974)

Beverley-Burton, 1984
Synonym: Cleidodiscus baldwini Dechtiar, 1971

Site: gills

Host: Percopsis omiscomaycus

Distribution: ON

Records: Dechtiar 1974a; Dechtiar \& Christie 1988; Dechtiar et al. 1988; Dechtiar \& Lawrie 1988 (ON); Dechtiar et al. 1989 (ON)

\section{? Urocleidus sp.}

Site: gills

Host: Etheostoma exile

Distribution: ON

Record: Molnar et al. 1974

\section{SUBFAMILY DACTYLOGYRINAE}

Acolpenteron catostomi Fischthal and Allison, 1942

Site: ureters

Hosts: Carpiodes cyprinus $(1,14)$ Catostomus catostomus $(3,4,7,8$, $9,12,13,15)$ Catostomus commersoni $(1,2,5,6$, $10,11,12,13,14,15)$ 9) Catostomus macrocheilus $(4,7,8$,

Distribution: $\quad \mathrm{BC}, \mathrm{NB}, \mathrm{ON}, \mathrm{QC}$ Records: 1) Dechtiar 1972a (ON); 2) Dechtiar 1972b (ON); 3) Hanek \& Molnar 1974 (QC); 4) Anonymous 1978 (BC); 5) Cone 1980 (NB); 6) Chan 1980 (ON); 7) Anonymous 1981 (BC); 8) Arai \& Mudry 1983 (BC); 9) Anonymous 1984 (BC); 10) Beverley-Burton 1984 (ON); 11) Dechtiar \& Christie 1988 
(ON); 12) Dechtiar et al.1988 (ON); 13)

Dechtiar \& Lawrie 1988 (ON); 14)

Dechtiar \& Nepszy 1988 (ON);

15) Dechtiar et al 1989 (ON)

Dactylogyrus albertensis Price and Arai, 1967

Site: gills

Host: Platygobio gracilis

Distribution: AB

Records: $\quad$ Price \& Arai 1967 (AB - Milk

River); Arai \& Chien 1973 (AB - Milk

River, Oldman River, St. Mary River)

Dactylogyrus banghami Mizelle and

Donahue, 1944

Site: gills

Hosts: Couesius plumbeus $(1,7,9)$ Margariscus margarita $(7,9)$

Rhinichthys cataractae $(1,2,3,4$, $5,6,7,8)$

Richardsonius balteatus (1)

Distribution: $\quad \mathbf{A B}, \mathrm{BC}, \mathrm{ON}$

Records: 1) Monaco \& Mizelle 1955

(BC, ON); 2) Price \& Arai 1967 (AB -

Milk River); 3) Dechtiar 1972a (ON); 4)

Hanek \& Fernando 1972b (ON); 5) Arai

\& Chien 1973 (AB - Milk River,

Oldman River, St. Mary River); 6)

Dechtiar \& Christie 1988 (ON); 7)

Dechtiar et al. 1988 (ON); 8) Dechtiar \&

Lawrie 1988 (ON); 9) Dechtiar et al.

1989 (ON)

Dactylogyrus buddi Dechtiar, 1974

Site: gills
Hosts: Cottus cognatus $(1,2,3,6)$

Cottus ricei $(4,5)$

Distribution: ON, YT

Records: 1) Dechtiar 1974b (ON); 2) Arthur et al. 1976 (YT); 3) Dechtiar \& Christie 1988 (ON); 4) Dechtiar et al. 1988 (ON); 5) Dechtiar \& Lawrie 1988 (ON); 6) Dechtiar et al. 1989 (ON)

Dactylogyrus bychowskyi Mizelle, 1937

Site: gills

Host: Pimephales promelas

Distribution: ON

Records: Hanek 1971; Molnar et al. 1974; Hanek et al. 1975; Chan 1980

Dactylogyrus chrosomi Molnar and Fernando, 1975

Site: gills

Hosts: Phoxinus eos $(1,3)$

Phoxinus neogaeus $(2,3)$

Distribution: $\quad \mathrm{ON}$

Records: 1) Hanek et al. 1975; 2)

Dechtiar et al. 1988; 3) Dechtiar et al. 1989

Dactylogyrus columbiensis Monaco and Mizelle, 1955

Site. gills

Host: Ptychocheilus oregonensis

Distribution: BC

Record: $\quad$ Monaco \& Mizelle 1955

Dactylogyrus duquesni (Mueller, 1938) Price, 1938 
Site: gills

Host: Moxostoma macrolepidotum

Distribution: ON

Record: Dechtiar et al. 1988

Dactylogyrus eos Hanek, Molnar and

Fernando, 1975

Site: gills

Host: Phoxinus eos

Distribution: NB, ON

Records: Hanek et al. 1975 (ON); Cone 1980 (NB); Dechtiar et al. 1989 (ON)

Dactylogyrus eucalius Mizelle and

Regensberger, 1945

Site: gills

Host: Culaea inconstans

Distribution: ON

Records: Dechtiar 1972a; Dechtiar

1972b; Hanek \& Fernando 1972b; Hanek

et al. 1975; Dechtiar \& Christie 1988;

Dechtiar et al. 1988; Dechtiar et al. 1989

Dactylogyrus hankinsoni Hanek, Molnar and

Fernando, 1975

Site: gills

Host: Hybognathus hankinsoni

Distribution: ON

Record: Hanek et al. 1975

Dactylogyrus mollis Chien, 1974

Site: gills

Host: Pimephales promelas

Distribution: ON
Record: Chan 1980

Dactylogyrus mylocheilus Monaco and Mizelle, 1955

Site: gills

Host: Couesius plumbeus

Distribution: BC, ON

Records: $\quad$ Monaco \& Mizelle 1955 (BC);

Dechtiar 1972a (ON)

Dactylogurus ptychocheilus Monaco and Mizelle, 1955

Site: gills

Host: Ptychocheilus oregonensis

Distribution: $\quad$ BC

Record: $\quad$ Monaco \& Mizelle 1955

Dactylogyrus richardsonius Monaco and Mizelle, 1955

Site: gills

Host: Richardsonius balteatus

Distribution: $\quad \mathrm{BC}$

Record: $\quad$ Monaco \& Mizelle 1955

Dactylogyrus tridactylus Monaco and Mizelle, 1955

Site: gills

Host: Ptychocheilus oregonensis

Distribution: $\quad$ BC

Record: $\quad$ Monaco \& Mizelle 1955

Dactylogyrus urus Mueller, 1938 
Site: gills

Hosts: Moxostoma anisurum (1) Moxostoma macrolepidotum (2)

Distribution: ON

Records: 1) Dechtiar 1972b; 2) Dechtiar \& Christie 1988

Dactylogyrus vancleavei Monaco and

Mizelle, 1955

Site: gills

Host: Ptychocheilus oregonensis

Distribution: $\mathrm{BC}$

Records: $\quad$ Monaco \& Mizelle 1955;

Anonymous 1981; Anonymous 1984

Dactylogyrus sp.

Site: gills

Hosts: Catostomus commersoni (4)

Culaea inconstans (3)

Etheostoma exile (8)

Gasterosteus aculeatus (5)

Hybognathus hankinsoni $(3,4)$

Margariscus margarita (6)

Moxostoma macrolepidotum (2)

Notropis atherinoides $(2,7,8)$

Notropis hudsonius $(1,2,7,8,9)$

Phoxinus neogaeus (7)

Pimephales promelas $(1,3,7)$

Distribution: BC, NB, ON

Records: 10 Dechtiar 1972a (ON); 2)

Dechtiar 1972b (ON); 3) Kakonge 1972

(ON); 4) Molnar et al. 1974 (ON); 5)

Lester 1975 (BC); 6) Cone 1980 (NB);

7) Dechtiar \& Christie 1988 (ON);

8) Dechtiar et al. 1988 (ON); 9) Dechtiar

\& Lawrie 1988 (ON)
Pellucidhaptor angularis Kritisky and Hathaway, 1969

Sites: body surface, fins

Host: Carpiodes cyprinus

Distribution: ON

Records: Dechtiar \& Nepszy 1988;

Beverley-Burton et al. 1992

Pellucidhaptor catostomi Dechtiar, 1969

Site: gills, nares

Host: Catostomus catostomus

Distribution: ON

Records: Dechtiar 1969; Dechtiar et al. 1988; Dechtiar \& Lawrie 1988; Dechtiar et al. 1989

Pellucidhaptor eremitus Rogers, 1967

Site: fins

Host: Carpiodes cyprinus

Distribution: ON

Record: ' Dechtiar \& Nepszy 1988

Pellucidhaptor eriensis Beverley-Burton, Chisolm and Dechtiar, 1992

Site: fins, body surface

Hosts: Carpiodes cyprinus

Distribution: ON

Moxostoma macrolepidotum

Record: Beverley-Burton et al. 1992

Pellucidhaptor micracanthus Kritsky, Leiby and Shelton, 1972

Site: fins 
Host: Carpiodes cyprinus

Distribution: ON

Records: Dechtiar \& Nepszy 1988;

Beverley-Burton et al. 1992

Pellucidhaptor moxostomi Mergo and

White, 1982

Site: fins, gills

Host: Moxostoma anisurum

Distribution: $\quad \mathrm{ON}$

Record: Beverley-Burton et al. 1992

Pellucidhaptor nasalis Dechtiar, 1969

Site: nares

Hosts: Catostomus catostomus $(5,6)$ $7,8,9)$

Catostomus commersoni $(1,2,3,4$,

Catostomus macrocheilus (6)

Distribution: BC, NB, ON

Records: 1) Dechtiar 1969 (ON); 2)

Dechtiar 1972a (ON); 3) Cone 1980

(NB); 4) Chan 1980 (ON); 5) Anonymous

1981 (BC); 6) Anonymous 1984 (BC); 7)

Dechtiar et al. $1988(\mathrm{ON}) ; 8)$ Dechtiar et

al. 1989 (ON); 9) Beverley-Burton et al. 1991 (ON)

Pellucidhaptor pellucidhaptor Price and Mizelle, 1964

Site: gills

Host: Richardsonius balteatus

Distribution: $\quad \mathrm{BC}$

Records: Anonymous 1978; Arai \&

Mudry 1983
Pellucidhaptor thelostea Beverley-Burton, Chisolm and Dechtiar, 1992

Site: body surface, fins

Host: Moxostoma macrolepidotum

Distribution: ON

Record: Beverley-Burton et al. 1992

Pellucidhaptor sp.

Sites: body surface, fins

Hosts: Carpiodes cyprimus (1)

Mo:iustoma macrolepidotum $(1,2)$

Distribution: ON

Records: 1) Dechtiar 1972b; 2) Dechtiar et al. 1988

Dactylogyridae gen. sp.

Site: [gills]

Host: Esox lucius

Distribution: $\mathbf{A B}$

Record: . Pybus \& Samuel 1978 (AB Carson Lake)

\section{FAMILY \\ PSEUDOMURRAYTREMATIDAE}

Anonchohaptor anomalum Mueller, 1938

Sites: fins, gills

Hosts: Carpiodes cyprinus $(1,2,11,15$,

17)

Catostomus catostomus $(3,4,5$,

$6,8,9,10,13,16)$

Catostomus commersoni $(1,2,3$,

$4,6,7,9,12,13,14,16,18)$

Catostomus macrocheilus $(6,9)$ 
12)

Moxostoma anisurum $(1,2)$

Moxostoma macrolepidotum (2,

Distribution: $\quad \mathrm{BC}, \mathrm{LB}, \mathrm{MB}, \mathrm{NB}, \mathrm{ON}$, QC, YT

Records: 1) Dechtiar 1972a (ON); 2)

Dechtiar 1972b (ON); 3) Dechtiar \&

Dillon 1974 (ON); 4) Threlfall 1974 (LB);

5) Arthur et al. 1976 (YT); 6)

Aponymous1978 (BC); 7) Cone 1980 (NB); 8) Anonymous 1981 (BC); 9) Arai \& Mudry 1981 (BC); 10) Anonymous 1984 (BC); 11) Szalai \& Dick 1987a (MB); 12) Dechtiar \& Christie 1988 (ON); 13) Dechtiar et al. 1988 (ON); 14) Dechtiar \& Lawrie 1988 (ON); 15) Dechtiar \& Nepszy 1988 (ON); 16) Dechtiar et al. 1989 (ON); 17) Szalai et al. 1992 (MB); 18) Dubois et al. 1996 (QC)

Anonchohaptor muelleri Kritsky, Leiby and Shelton, 1972

Site: fins

Host: Carpiodes cyprinus

Distribution: ON

Record: Dechtiar \& Nepszy 1988

Anonchohaptor sp.

Site: [gills]

Host: Carpiodes cyprinus

Distribution: ON

Record: Dechtiar 1972b

Icelanonchohaptor fyviei Dechtiar and Dillon, 1974
Sites: body surface, fins

Host: Carpiodes cyprinus

Distribution: ON

Records: Dechtiar \& Dillon 1974;

Dechtiar \& Nepszy 1988

Icelanonchohaptor microcotyle Kritsky,

Leiby and Shelton, 1972

Sites: body surface, fins

Host: Carpiodes cyprinus

Distribution: $\mathrm{ON}$

Record: Dechtiar \& Nepszy 1988

Pseudomurraytrema alabarum Rogers, 1966

Site: gills

Host: Catostomus commersoni

Distribution: ON

Record: Dechtiar et al. 1989

Pseudomurraytrema copulatum (Mueller, 1938) Bychowsky, 1957

Site: gills

Hosts: Catostomus catostomus $(3,4,5,6$, 9) Catostomus commersoni $(1,2,3$, $5,7,9,10,11)$

6) Moxostoma anisurum $(1,2)$

Catostomus macrocheilus $(3,4,5$,

Moxostoma macrolepidotum $(7,8)$

Distribution: $\mathrm{BC}, \mathrm{ON}$

Records: 1) Dechtiar 1972a (ON); 2)

Dechtiar 1972b (ON); 3) Anonymous

1978 (BC); 4) Anonymous 1981 (BC); 5)

Arai \& Mudry 1983 (BC); 6) Anonymous 1984 (BC); 7) Dechtiar \& Christie 1988 
(ON); 8) Dechtiar et al. 1988 (ON); 9)

Dechtiar \& Lawrie 1988 (ON); 10)

Dechtiar \& Nepszy 1988 (ON); 11)

Dechtiar et al.1989(ON)

Pseudomurraytrema muelleri Price, 1967

Site: gills

Host: Catostomus commersoni

Distribution: $\quad \mathbf{A B}, \mathrm{ON}$

Records: Price \& Arai 1967 (AB - Milk

River); Chan 1980 (ON)

Pseudomurraytrema sp.

Site: gills

Host: Moxostoma anisurum

Distribution: ON

Record: Dechtiar 1972a

\section{SUBORDER TETRAONCHINEA}

\section{FAMILY TETRAONCHIDAE}

Tetraonchus alaskensis Price, 1937

Sice: gills

Hosts: Oncorhynchus kisutch $(2,4)$ Salvelinus malma $(1,2,3,4)$

Distribution: $\quad \mathrm{BC}$

Records: 1) Anonymous 1978; 2) Anonymous 1981; 3) Arai \& Mudry 1983;

4) Anonymous 1984

Tetraonchus borealis (Olsson, 1893)

Monticelli, 1905
Site: gills

Host: Thymallus arcticus

Distribution: BC, SK, YT

Records: 1) Arthur et al. 1976 (YT); 2) Wobeser et al. 1976 (SK); 3) Anonymous 1978 (BC); 4) Arai \& Mudry 1983 (BC)

Tetraonchus loftusi Dechtiar, 1972

Site: gills

Hosts: Esox lucius

Prosopium cylindraceum

Distribution: ON

Record: Dechtiar 1972c

Tetraonchus monenteron (Wagener, 1857) Diesing, 1858

Site: gills

Hosts: Esox lucius $(1,2,3,4,5,6,7,8,9$, $10,11,12,13,14,15,16,17,18,19,20$, $21,22,23)$

\section{Perca flavenscens (9)}

Salvelinus namaycush (12)

Distribution: $\quad$ AB , LB, MB, ON, YT

Records: $\quad 1)$ Threlfall \& Hanek $1970 \mathrm{~b}$ (LB); 2) Hanek 1971 (ON); 3) Hanek \& Fernando 1972a (ON); 4) Hanek \& Fernando 1972b (ON); 5) Dechtiar 1972a (ON); 6) Dechtiar 1972b (ON); 7) Dechtiar 1972c (ON); 8) Arai \& Chien 1973 (AB - Milk River, Oldman River, St. Mary River); 9) Hicks \& Threlfall 1973 (LB); 10) Arthur et al. 1976 (YT); 11) Watson 1977 (MB); 12) Chinniah \& Threlfall 1978 (LB); 13) Pybus \& Samuel 1978 (AB - Blue Lake, Cache Lake, Jarvis Lake); 14) Watson \& Dick 1980 (MB); 15) Samuel 1981 (AB - Blue Lake, Cache Lake, Jarvis Lake); 16) 
Anthony 1983 (ON); 17) Samuel 1985 (AB - Blue Lake, Cache Lake, Jarvis Lake); 18) Dechtiar \& Christie 1988 (ON); 19) Dechtiar et al. 1988 (ON); 20) Dechtiar \& Lawrie 1988 (ON); 21) Dechtiar \& Nepszy 1988 (ON); 22) Stock 1988 (AB - Jarvis Lake); 23) Szalai et al. $1992(\mathrm{MB})$

Tetraonchus variabilis Mizelle and Webb, 1953

Site: gills

Hosts: Coregonus clupeaformis (8) Oncorhychus mykiss (8)

Prosopium coulteri (4)

Prosopium cylindraceum (1, 2, 3, $9,10,11)$

Prosopium williamsoni $(5,6,7,8)$

Salvelinus fontinalis (2)

Distribution: $\quad$ BC, LB, ON, YT

Records: 1) Dechtiar 1972c (ON); 2)

Hicks \& Threlfall 1973 (LB); 3) Arthur et al. 1976 (YT); 4) Mudry \& Anderson 1977 (BC); 5) Anonymous 1978 (BC); 6) Anonymous 1981 (BC); 7) Arai \& Mudry 1983 (BC); 8) Anonymous 1984 (BC); 9)

Dechtiar et al. 1988 (ON); 10) Dechtiar \& Lawrie 1988 (ON); 11) Dechtiar et al. 1989 (ON)

Tetraonchus sp.

Site: [gills]

Host: Stizostedion vitreum

Distribution: $\quad$ AB

Records: Fillion 1961 (AB - Lac SteAnne); Ryder 1963 (AB - Iosegun Lake)

\section{ORDER GYRODACTYLIDEA}

\section{FAMILY GYRODACTYLIDAE}

Gyrodactyus aldrichi Threlfall, 1974

Site: gills

Host: Couesius plumbeus

Distribution: LB

Record: Threlfall 1974

Gyrodactylus alexanderi Mizelle and Kritsky, 1967

Site: body surface, fins

Host: Gasterosteus aculeatus

Distribution: BC

Records: Lester 1971a; Lester 1972; Lester \& Adams 1974a; Lester \& Adams 1974b; Lester 1975; Cone \& Wiles 1985

Gyrodactylus aquilinus Threlfall, 1974

Site: fins

Host: Catostomus catostomus

Distribution: LB, ON

Records: Threlfall 1974 (LB); Chan 1980 (ON)

Gyrodactylus atratuli Putz and Hoffman, 1963

Site: fins

Hosts: Catostomus catostomus (4) Catostomus commersoni (5) Rhinichthys cataractae $(1,2,3,6)$

Distribution: LB, ON 
Records: 1) Hanek \& Fernando 1971 (ON); 2) Hanek 1971 (ON); 3) Hanek \& Fernando 1972b (ON); 4) Threlfall 1974 (LB); 5) Chan 1980 (ON); 6) Dechtiar et al. $1988(\mathrm{ON})$

Gyrodactylus avalonia Hanek and Threlfall, 1969

Synonyms: Gyrodactylus lairdi Hanek and Threlfall, 1969; Gyrodactylus memorialis Hanek and Threlfall, 1969; Gyrodactylus terranovae Hanek and Threlfall, 1969

Site: body surface

Hosts: Gasterosteus aculeatus (1, 2, 5, 6, $7,10,11,12,13)$ Oncorhynchus mykiss (9) Pungitius pungitius $(3,8,12,14)$ Salvelinus namaycush (4)

Distribution: $\quad$ LB, NB, NF, NS, ON, QC Records: 1) Hanek \& Threlfall $1969 \mathrm{~b}$ (NF); 2) Hanek \& Threlfall 1970a (NF); 3) Hanek \& Threlfall 1970b (LB); 4) Threlfall \& Hanek 1970c (NF); 5) Hanek \& Fernando 1971 (ON); 6) Hanek 1971 (ON); 7) Hanek \& Fernando 1972b (ON); 8) Hanek \& Molnar 1974 (QC); 9) Cone et al. 1982 (NS); 10) Cone \& Odense 1984 (NF); 11) Cone \& Ryan 1984 (NF); 12) Cone \& Wiles 1985 (NB, NF, NS, ON); 13) Dechtiar \& Christie 1988 (ON); 14) Marcogliese 1995 (NS)

Gyrodactylus bairdi Wood and Mizelle, 1957

Synonym: Gyrodactylus labradorius Hanek and Threlfall, 1970

Site: fins
Host: Cottus cognatus

Distribution: ON, YT

Records: $\quad$ Arthur et al. 1976 (YT);

Dechtiar et al. 1989 (ON)

Gyrodactylus calliaratus Malmberg, 1957

Site: body surface, gills

Host: Salvelinus fontinalis

Distribution: NB

Records: Frimeth 1987a; Frimeth 1987b

Gyrodactylus canadensis Hanek and Threlfall, 1969

Site: giiis

Hosts: Gasterosteus aculeatus $(1,2,5)$ Pungitius pungitius $(3,4,5,6,7)$

Distribution: LB, NB, NF, NS, NT, QC Records: 1) Hanek \& Threlfall 1969b (NF); 2) Hanek \& Threlfall 1970a (NF); 3) Hanek \& Threlfall 1970b (NF); 4) Hanek \& Molnar 1974 (QC); 5) Cone \& Wiles 1985 (NB, NF, NS, NT); 6) Marcogliese 1992 (NS)

Gyrodactylus colemanensis Mizelle and Kritsky, 1967

Site: body surface, fins

Hosts: Oncorhynchus mykiss (1, 2, 5, 6, 7, $8,9)$

Salmo trutta (1)

Salvelinus fontinalis $(1,2,3,4,5$, $7,9)$

Salvelinus fontinalis X Salvelinus namaycush (1)

Salvelinus namaycush (1)

Distribution: NB, NF, NS, ON 
Records: 1) Cone et al. 1983 (NF, NS, ON); 2) Cusack 1986 (NS); 3) Frimeth 1987a (NB); 4) Frimeth 1987b (NB); 5) Cone \& Cusack 1988 (NS); 6) Cusack et al. 1988 (NS); 7) Cone \& Cusack 1989 (NS); 8) Cone \& Wiles 1989 (NS); 9) Wells \& Cone 1990 (NS)

Gyrodactylus commersoni Threlfall, 1974

Site: gills

Host: Catostomus commersoni

Distribution: LB

Record: Threlfall 1974

Gyrodactylus couesius Wood and Mizelle, 1954

Sites: fins, gills

Host: Couesius plumbeus

Distribution: BC, ON

Records: Wood \& Mizelle 1957 (BC); Dechtiar et al. 1988 (ON); Dechtiar et al. 1989 (ON)

Gyrodactylus dechtiari Hanek and

Fernando, 1971

Sites: fins, gills

Host: Rhinichthys cataractae

Distribution: ON

Records: Hanek \& Fernando 1971:

Hanek 1971; Hanek \& Fernando 1972b; Dechtiar et al. 1988; Dechtiar \& Lawrie 1988

Gyrodactylus etheostomae Wellborn and Rogers, 1967
Sites: fins, gills

Host: Etheostoma exile

Distribution: ON

Record: $\quad$ Molnar et al. 1974

Gyrodactylus eucaliae Ikezaki and Hoffman, 1957

Sites: body surface, fins

Hosts: Culaea inconstans $(1,2,3,4,5,6$, 7,8 )

Pungitius pungitius (7)

Distribution: ON

Records: 1) Hanek \& Fernando 1971; 2) Hanek \& Fernando 1972b; 3) Dechtiar 1972a; 4) Kakonge 1972: 5) Cone \&Wiles 1985; 6) Dechtiar \& Christie 1988; 7) Dechtiar et al. 1988; 8) Dechtiar et al. 1989

Gyrodactylus freemani Hanek and Fernando, 1971

Site: fins

Host: Perca flavescens

Distribution: ON

Records: Hanek \& Fernando 1971; Hanek \& Fernando 1972b; Dechtiar \& Christie 1988; Dechtiar et al. 1988; Dechtiar et al. 1989

Gyrodactylus hoffmani Wellborn and Rogers, 1967

Site: fins

Host: Pimephales promelas

Distribution: ON

Records: Kakonge 1972; Molnar et al. 1974; Chan 1980 
Gyrodactylus lacustris Mizelle and Kritsky, 1967

Site: fins

Host: Pimephales promelas

Distribution: ON

Record: Dechtiar et al. 1989

Gyrodactylus limi Wood and Mizelle, 1957

Sites: body surface, fins

Host: Catostomus commersoni

Distribution: ON

Record: Kakonge 1972

Gyrodactylus lotae Gusev, 1953

Site: body surface

Host: Lota lota

Distribution: ON

Record: Cone \& Dechtiar 1986

Gyrodactylus lucii Kulakovskaya, 1952

Site: body surface

Host: Esox lucius

Distribution: ON

Record: Cone \& Dechtiar 1986

Gyrodactylus margaritae Putz and Hoffman, 1963

Site: fins

Host: Margariscus margarita

Distribution: ON

Record: Dechtiar et al. 1988
Gyrodactylus perlucidus Bykhovsky and Polyansky, 1953

Sites: body surface, fins

Host: Salvelinus fontinalis

Distribution: NB

Records: Frimeth 1987a; Frimeth 1987b

Gyrodactylus plumbeae Threlfall, 1974

Site: gills

Host: Couesius plumbeus

Distribution: LB

Record: Threlfall 1974

Gyrodactylus salmonis Yin and Sproston, 1948

Sites: body surface, gills

Hosts: Oncorhynchus clarki (1)

Oncorhynchus mykiss $(1,5,6)$

Salvelinus fontinalis $(1,2,3,4,5$,

6)

Distribution: $\quad$ BC, NB, NS

Records: 1) Cone et al. 1983 (BC, NS);

2) Cusack \& Cone 1986 (NS); 3) Frimeth 1987a (NB); 4) Frimeth 1987b (NB); 5)

Cone \& Cusack 1988 (NS); 6) Wells \& Cone 1990 (NS)

Gyrodactylus spathulatus Mueller, 1936

Sites: fins, gills

Hosts: Catostomus catostomus (3)

$$
5,6,7,8)
$$

Catostomus commersoni $(1,2,4$,

Moxostoma anisurum (1)

Distribution: LB, ON

Records: 1) Dechtiar 1972a (ON); 2) 
Dechtiar 1972b (ON); 3) Threlfall 1974 (LB); 4) Chan 1980 (ON); 5) Dechtiar \& Christie 1988 (ON); 6) Dechtiar et al. 1988 (ON); 7) Dechtiar \& Nepszy 1988 (ON); 8) Dechtiar et al. 1989 (ON)

Gyrodactylus stephanus Mueller, 1937

Site: gills

Host: Pungitius pungitius

Distribution: NF

Record: Dickinson \& Threlfall 1976

Gyrodactylus stunkardi Kritsky and Mizelle, 1968

Sites: fins, gills

Hosts: Catostomus commersoni (5)

Rhinichthys cataractae $(1,2,3,4$,

6)

Distribution: LB, ON

Records: 1) Hanek \& Fernando 1971

(ON); 2) Hanek 1971 (ON); 3) Hanek \&

Fernando 1972b (ON); 4) Threlfall 1974

(LB); 5) Molnar et al. 1974 (ON); 6)

Dechtiar \& Christie 1988 (ON)

Gyrodactylus sp.

Sites: body surface, fins, gills

Hosts: Carpiodes cyprinus (20)

Catostomus catostomus (21)

Catostomus commersoni $(17,18$,

19)

Culaea inconstans (2)

Esox lucius (18)

Etheostoma exile $(1,3,18)$

Gasterosteus aculeatus (17)

Hybognathus hankinsoni $(3,6)$
Lota lota (21)

Moxostoma anisurum (2)

Moxostoma macrolepidotum (2,

17)

Notropis atherinoides (18)

Notropis hudsonius $(2,17,18)$

Oncorhynchus clarki (8)

Oncorhynchus kisutch (13)

Oncorhynchus mykiss $(5,9,14)$

Perca flavescens (3)

Percina caprodes (18)

Percopsis omiscomaycus $(1,17,18$

21)

Phoxinus eos $(10,21)$

Phoxinus neogaeus $(17,18,21)$

Pimephales promelas $(3,17,18)$

Ptychocheilus oregonensis (15)

Pungitius pungitius $(7,11,12,16)$

Salvelinus fontinalis $(4,5)$

Distribution: $\quad$ AB, BC, LB, NB, NS, NT, ON

Records: 1) Dechtiar 1972a (ON); 2)

Dechtiar 1972b (ON); 3) Kakonge 1972

(ON); 4) Hicks \& Threlfall 1973 (LB); 5)

Hare \& Frantsi 1974 (NS); 6) Molnar et

al. 1974 (ON); 7) Leong 1975 (AB -

Cold Lake); 8) Hoskins et al. 1976 (BC);

9) Hoskins \& Hulstein 1977 (BC); 10)

Cone 1980 (NB); 11) Leong \& Holmes

1981 (AB - Cold Lake); 12) Curtis 1982

(NT); 13) Margolis 1982 (BC); 14)

McAllister \& Mudry 1983 (AB - Sam

Livingstone Hatchery); 15) Cone \&

Dechtiar 1984 (BC); 16) Cone \& Wiles 1985 (ON); 17) Dechtiar \& Christie 1988

(ON); 18) Dechtiar et al. 1988 (ON); 19)

Dechtiar \& Lawrie 1988 (ON); 20)

Dechtiar \& Nepszy 1988 (ON); 21)

Dechtiar et al. 1989 (ON) 
Gyrodactylidae gen. sp.

Site: gills

Hosts: Catostomus commersoni

Perca flavescens

Phoxinus eos

Distribution: ON

Record: Bangham 1941

Gyrodactyloidea gen. sp.

Sites: body surface, gills

Hosts: Catostomus catostomus (2)

Catostomus commersoni (1)

Catostomus macrocheilus (2)

Cottus cognatus (2)

Couesius plumbeus (2)

Culaea inconstans $(2,3)$

Esox lucius (3)

Gasterosteus aculeatus (2)

Notropis atherinoides (3)

Notropis hudsonius (3)

Oncorhynchus clarki (2)

Oncorhynchus kisutch (2)

Oncorhynchus mykiss (2)

Perca flavescens $(1,3)$

Percina caprodes (3)

Percopsis omiscomaycus (3)

Phoxinus eos (3)

Phoxinus neogaeus (1)

Pimephales promelas (3)

Prosopium cylindraceum (2)

Prosopium williamsoni $(2,4,5)$

Ptychocheilus oregonensis (2, 4,

5)

Rhinichthys cataractae (2)

Richardsonius balteatus (2)

Salvelinus malma (2)

Stizostedion vitreum (3)

Distribution: $\quad \mathrm{BC}, \mathrm{ON}$

Records: 1) Bangham 1941 (ON); 2)
Bangham \& Adams 1954 (BC); 3)

Bangham 1955 (ON); 4) Anonymous

1978 (BC); 5) Arai \& Mudry 1983 (BC)

ORDER DICLYBOTHRIIDEA

\section{FAMILY DICLYBOTHRIIDAE}

Diclybothrium atriatum Choudhury and Dick, 1996

Synonym: Diclybothrium armatum Leuckart, 1835

Site: gills

Host: Acipenser fulvescens

Distribution: $\quad \mathrm{MB}, \mathrm{ON}, \mathrm{QC}, \mathrm{SK}$

Records: Stafford 1904 (ON); Cooper 1915 (ON); Price 1942 (QC); Bangham 1955 (ON); Dechtiar 1972a (ON); Dechtiar 1972b (ON); Anthony 1974 (ON); Wright \& Dechtiar 1974 (ON); Dechtiar \& Christie 1988 (ON); Dechtiar et al. $1988(\mathrm{ON})$; Dechtiar \& Lawrie 1988 (ON); Choudhury et al 1990 (MB, SK); Swanson et al. 1991 (MB); Choudhury \& Dick 1993 (MB, SK); Choudhury \& Dick 1996b (MB, SK)

Diclybothrium hammulatum (Simer, 1929)

Price, 1942

Synonym: Diplobothrium hammulatum Simer, 1929

Site: gills

Host: Acipenser fulvescens

Distribution: ON

Record: Bangham 1955 
Paradiclybothrium sp.

Site: gills

Host: Acipenser fulvescens

Distribution: ON

Record: Anthony 1974

ORDER MAZOCRAEIDA

SUBORDER DISCOCOTYLINEA

FAMILY DISCOCOTYLIDAE

Discocotyle sagittata (Leuckart, 1842) Diesing, 1850

Synonym: Discocotyle salmonis Schaffer, 1916

Site: gills

Hosts: Coregonus artedii $(2,6,10,11,13$, $15,16,17,20)$

Coregonus clupeaformis $(6,8,10$,

$11,12,13,14,16,17,18,19,20,22)$

Oncorhynchus kisutch $(10,11,17)$

Oncorhynchus mykiss (7)

Prosopium cylindraceum $(2,8,12$,

20)

Prosopium williamsoni (1)

Salmo trutta $(3,4,5,7)$

Salvelinus fontinalis $(3,4,5,7,8$, $9,14,18,21)$

Salvelinus fontinalis X Salvelinus namaycush $(15,19)$

Salvelinus malma (1)

20)

Salvelinus namaycush $(8,10,12$,

Thymallus arcticus (12)

Distribution: $\quad \mathrm{AB}, \mathrm{BC}, \mathrm{LB}, \mathrm{MB}, \mathrm{NF}, \mathrm{NS}$,
ON, YT

Records: 1) Bangham \& Adams 1954

(BC); 2) Bangham 1955 (ON); 3)

Sandeman \& Pippy 1967 (NF); 4) Hanek \& Threlfall 1970c (NF); 5) Hanek 1971 (ON); 6) Dechtiar 1972a (ON); 7) Hanek \& Fernando $1972 \mathrm{~b}(\mathrm{ON}) ; 8)$ Hicks \& Threlfall 1973 (LB); 9) Hare \& Frantsi 1974 (NS); 10) Leong \& Holmes 1974a (AB - Cold Lake); 11) Leong 1975 (ABCold Lake); 12) Arthur et al. 1976 (YT); 13) Watson 1977 (MB); 14) Chinniah \& Threlfall 1978 (LB); 15) Dechtiar \& Berst 1978 (ON); 16) Watson \& Dick 1979 (MB); 17) Leong \& Holmes 1981 (AB Cold Lake); 18) Cone \& Ryan 1984 (NF); 19) Dechtiar et al. 1988 (ON); 20) Dechtiar \& Lawrie 1988 (ON); 21) Marcogliese \& Cone 1991b (NF)

Discocotyle sp.

Site: gills

Host: Salvelinus fontinalis

Distribution: QC

Record: Hanek \& Molnar 1974

Neodiscocotyle carpioditis Dechtiar, 1967

Site: gills

Host: Carpiodes cyprinus

Distribution: ON

Records: Dechtiar 1967b; Dechtiar 1972; Wright \& Dechtiar 1974; Dechtiar \& Nepszy 1988

FAMILY OCTOMACRIDAE 
Octomacrum lanceatum Mueller, 1934

Site: gills

Hosts: Catostomus catostomus $(4,10,12$,

$14,17,18,20)$

Catostomus commersoni $(1,2,3,5$,

$6,7,8,11,16,17,18,19$ )

Catostomus macrocheilus $(4,9,12$,

13, 14)

Pimephales promelas (15)

Distribution: $\mathrm{BC}, \mathrm{ON}$

Records: 1) Wright 1879 (ON); 2)

Bangham 1941 (ON); 3) Bangham \&

Venard 1946 (ON); 4) Bangham \&

Adams 1954 (BC); 5) Bangham 1955

(ON); 6) Dechtiar 1972a (ON); 7)

Dechtiar 1972b (ON); 8) Molnar et al.

1974 (ON); 9) Anonymous 1978 (BC);

10) Shepherd \& Mace 1980 (BC); 11)

Chan 1980 (ON); 12) Anonymous 1981

(BC); 13) Arai \& Mudry 1983 (BC); 14)

Anonymous 1984 (BC); 15) Beverley-

Burton 1984 (ON); 16) Dechtiar \&

Christie 1988 (ON); 17) Dechtiar et al.

1988 (ON); 18) Dechtiar \& Lawrie 1988

(ON); 19) Dechtiar \& Nepszy 1988 (ON);

20) Dechtiar et al. 1989 (ON)

Octomacrum microconfibula Hargis, 1952

Site: gills

Hosts: Margariscus margarita (1)

Phoxinus eos (1)

Richardsonius balteatus (2)

Distribution: $\quad$ BC, NB

Records: 1) Cone 1980 (MB); 2)

Shepherd \& Mace 1980 (BC)

Octomacrum semotili Dechtiar, 1966
Site: gills

Hosts: Couesius plumbeus $(2,3,4)$

Margariscus margarita (1)

Notropis hudsonius (3)

Phoximus eos (4)

Phoxinus neogaeus $(1,2,4)$

Pimephales promelas (4)

Distribution: ON

Records: 1) Dechtiar 1972a; 2) Dechtiar \& Christie 1988; 3) Dechtiar et al. 1988;

4) Dechtiar et al. 1989

Octomacrum sp.

Site: gills

Hosts: Couesius plumbeus $(3,4)$

Hybognathus hankinsoni (1)

Margariscus margarita (2)

Richardsonius balteatus (3)

Distribution: $\quad \mathrm{BC}, \mathrm{ON}$

Records: 1) Bangham 1941 (ON); 2)

Bangham \& Venard 1946 (ON); 3)

Bangham \& Adams 1954 (BC); 4)

Dechtiar 1972a (ON)

SUBORDER MAZOCRAEOIDEA

FAMILY MAZOCRAEIDAE

Mazocraedoides sp.

Site: gills

Host: Hiodon tergisus

Distribution: ON

Record: Dechtiar 1972b 
Monogenea gen. sp.

Site: gills

Hosts: Catostomus catostomus $(1,4)$

Oncorhynchus mykiss (3)

Perca flavescens $(2,5)$

Distribution: AB, BC

Records: 1) Anonymous 1981 (BC); 2)

Samuel 1981 (AB - Jarvis Lake); 3)

McAllister \& Mudry 1983 (AB - Hasse

Lake); 4) Anonymous 1984 (BC); 5)

Samuel 1985 (AB - Jarvis Lake)

CLASS TREMATODA

SUBCLASS DIGENEA

ORDER STRIGEIFORMES

SUPERFAMILY CLINOSTOMOIDEA

FAMILY CLINOSTOMIDAE

*Clinostomum complanatum (Rudolphi, 1814) Braun, 1899

Synonym: Clinostomum marginatum

(Rudophi, 1814) Braun, 1899

Includes: ?Clinostomum gracile of Stafford (1904)

Sites: fins, gills, gill chamber, mesenteries, musculature

Hosts: Catostomus commersoni $(6,9,25$, 30)

Culaea inconstans (19)

Esox lucius $(5,9,20)$
Etheostoma exile (19)

Hybognathus hankinsoni (19)

Notropis hudsonius $(20,28)$

Oncorhynchus mykiss $(23,29)$

Perca flavescens $(1,2,3,4,5,6$, $8,9,10,13,14,15,16,17,18,20,21$, $22,24,26,27)$

Percina caprodes (4)

Phoxinus eos $(27,30)$

Pimephales promelas $(8,19)$

Richardonius balteatus (12)

Salvelinus fontinalis $(7,9,18)$

Stizostedion canadense (20)

Stizostedion vitreum $(11,14,15$,

$16,18,20,24,26)$

Distribution: $\quad \mathrm{BC}, \mathrm{MB}, \mathrm{ON}, \mathrm{QC}$

Records: 1) Wright 1879 (ON); 2)

Stafford 1904 (QC); 3) Cooper 1915

(ON); 4) Bangham \& Hunter 1939 (ON);

5) Lyster 1939 (QC); 6) Bangham 1941

(ON); 7) MacLulich 1943b (ON); 8)

Bangham \& Venard 1946 (ON); 9)

Fantham \& Porter 1948 (QC); 10) Worley \& Bangham 1952 (QC); 11) Stewart-Hay 1952a (MB); 12) Bangham \& Adams 1954 (BC); 13) Bangham 1955 (ON); 14) Evans 1963b (MB); 15) Dickson 1964 (MB); 16) Dowsett \& Lubinsky 1966 (MB); 17) Tedla (ON); 18) Tedla \& Fernando 1969d (ON); 19) Kakonge 1972 (ON); 20) Dechtiar 1972a (ON); 21) Molnar et al. 1974 (ON); 22) Anthony 1976 (ON); 23) Hoskins et al. 1976 (BC); 24) Anthony 1978 (ON); 25) Chan 1980 (ON); 26) Dechtiar \& Christie 1988

(ON); 27) Dechtiar et al. 1988 (ON); 28) Dechtiar \& Lawrie 1988 (ON); 29) Szalai \& Dick 1988 (MB); 30) Dechtiar et al. 1989 (ON) 
*Clinostomum sp.

Sites: musculature, viscera

Hosts: Oncorhynchus clarki (3)

Oncorhynchus mykiss $(1,2,3)$

Perca flavescens $(1,2,4)$

Phoxinus eos (5)

Stizostedion vitreum (2)

Distribution: $\quad \mathrm{BC}, \mathrm{MB}, \mathrm{NB}, \mathrm{QC}$

Records: 1) McLeod 1943 (MB); 2)

Dickson 1964 (MB); 3) Hoskins et al. 1976 (BC); 4) Pike \& Burt 1981 (NB); 5)

Dubois et al. 1996 (QC)

SUPERFAMILY STRIGEOIDEA

\section{FAMILY DIPLOSTOMIDAE}

*Crassiphialia bulboglossa Haitsma, 1925

Sites: fins, musculature, subcutaneous

Hosts: Couesius plumbeus (11)

Esox lucius $(1,2)$

Notropis hudsonius $(4,9)$

Perca flavescens $(1,2,3,5,6,7$,

$8,10)$

Phoxinus eos (12)

Pimephales promelas $(3,4,7,11)$

Rhinichthys cataractae $(8,9)$

Stizostedion vitreum $(3,8,10)$

Distribution: $\quad \mathrm{MB}, \mathrm{ON}, \mathrm{QC}$

Records: 1) Miller 1940a (QC); 2)

Cameron 1946 (QC); 3) Crossman 1962

(ON); 4) Kask 1963 (MB); 5) Dechtiar

1972a (ON); 6) Dechtiar 1972b (ON); 7)

Dechtiar \& Christie 1988 (ON); 8)

Dechtiar et al. 1988 (ON); 9) Dechtiar \&

Lawrie 1988 (ON); 10) Dechtiar \&

Nepszy 1988 (ON); 11) Dechtiar et al.
$1989(\mathrm{ON})$

*Neascus pyriformis Chandler, 1951

Sites: fins, subcutaneous

Host: Perca flavescens

Distribution: ON

Record: Molnar et al. 1974

*Neascus rhinichthysi Hunter, 1933

Sites: muscilature, subcutaneous

Host: Rhinichthys cataractae

Distribution: ON

Record: Bangham \& Hunter 1939

*Neascus sp.

Sites: fins, gills, mesenteries, subcutaneous

Hosts: Catostomus catostomus $(4,10,15$, $18,22)$

Catostomus commersoni $(5,6,7$,

$11,16,20,21,23)$

Catostomus macrocheilus (4)

Coregomus artedii (6)

Coregonus clupeaformis $(6,7)$

Cottus cognatus (14)

Couesius plumbeus $(4,11)$

Culaea inconstans $(5,11,12)$

Esox lucius $(6,7,23)$

Etheostoma exile $(11,12)$

Hybognathus hankinsoni $(3,12)$

Lota lota $(6,7)$

Margariscus margarita (3)

Moxostoma anisurum $(11,23)$

Moxostoma macrolepidotum (1)

Notropis hudsonius $(1,5,10,11$, 
Oncorhynchus clarki (4)

Oncorhynchus mykiss (13)

Perca flavescens $(1,3,4,5,6,7$,

$8,9,10,22$ )

Percina caprodes $(1,11,23)$

Percopsis omiscomaycus $(5,7)$

Phoxinus eos (4)

Pimephales promelas $(1,11,12$,

$16,20,21)$

Ptychocheilus oregonensis (4, $15,17,18,19)$

Rhinichthys cataractae $(4,11$, 21)

Richardsonius balteatus (4)

Salvelinus fontinalis $(2,4,21)$

Salvelinus malma (4)

Stizostedion canadense $(1,11)$

Stizostedion vitreum $(1,5,23)$

Distribution: $\quad \mathbf{A B}, \mathrm{BC}, \mathrm{MB}, \mathrm{ON}, \mathrm{QC}, \mathrm{YT}$ Records: 1) Bangham \& Hunter 1939 (ON); 2) MacLulich 1943b (ON); 3)

Bangham \& Venard 1946 (ON); 4)

Bangham \& Adams 1954 (BC); 5)

Bangham 1955 (ON); 6) Lawler 1965e

(MB); 7) Lawler 1966c (MB); 8) Tedla \&

Fernando 1969a (ON); 9) Tedla 1969

(ON); 10) Paetz \& Nelson 1970 (AB -

unspecified); 11) Dechtiar 1972a (ON);

12) Kakonge 1972 (ON); 13) Hoskins et

al. 1976 (BC); 14) Arthur et al. 1976

(YT); 15) Anonymous 1978 (BC); 16)

Chan 1980 (ON); 17) Anonymous 1981

(BC); 18) Arai \& Mudry 1983 (BC); 19)

Anonymous 1984 (BC); 20) Dechtiar et

al.1988 (ON); 21) Steedman 1991 (ON);

22) Nelson \& Paetz 1992 (AB -

unspecified); 23) Szalai et al. 1992 (MB)

*Ornithodiplostomum ptychocheilus (Faust, 1917) Dubois, 1936
Sites: central nervous system, eye orbit, musculature, viscera

Hosts: Catostomus commersoni (1) Hybognathus hankinsoni $(1,2)$

Perca flavescens (3)

Pimephales promelas $(1,2,4)$

Stizostedion vitreum (3)

Distribution: MB, ON

Records: 1) Kakonge 1972 (ON); 2)

Molnar et al. 1974 (ON); 3) Poole \& Dick 1985 (MB); 4) Chan 1980 (ON)

*Posthodiplostomum cuticola (Nordmann, 1832) Dubois, 1936

Sites: kidney, liver, mesenteries

Hosts: Notropis atherinoides Percina caprodes

Distribution: ON

Record: Cooper 1915

*Posthodiplostomum minimum

(MacCallum, 1921) Dubois, 1936

Synonym: Neascus vancleavei

(Agersborg, 1926)

Sites: kidney, liver, mesenteries

Hosts: Carpiodes cyprinus (12)

Catostomus catostomus $(4,5,7$,

8)

Catostomus commersoni $(3,12)$

Catostomus macrocheilus (4)

Couesius plumbeus $(4,5,10)$

Culaea inconstans $(5,10)$

Esox lucius (6)

Etheostoma exile (5)

Gasterosteus aculeatus (4)

Lota lota $(4,5)$

INotropis atherinoides $(1,5,10)$

Notropis hudsonius $(1,5,6,10$, 
11)

Perca flavescens $(2,3,5)$

Percina caprodes (6)

Percopsis omiscomaycus $(1,10)$

Phoxinus eos $(3,5)$

Phoxinus neogaeus $(6,10)$

Pimephales promelas $(3,6)$

Ptychocheilus oregonensis (4, 7,

8)

10,11)

Rhinichthys cataractae $(4,5,6$,

Richardsonius balteatus $(4,8)$

Salvelinus fontinalis (5)

Stizosteciion vit:eum (1)

Distribution: $\quad \mathrm{BC}, \mathrm{ON}$

Records: 1) Bangham \& Hunter 1939

(ON); 2) Bangham 1941 (ON); 3)

Bangham \& Venard 1946 (ON); 4)

Bangham \& Adams 1954 (BC); 5)

Bangham 1955 (ON); 6) Dechtiar 1972a

(ON); 7) Anonymous 1981 (BC); 8)

Anonymous 1984 (BC); 9) Dechtiar \&

Christie 1988 (ON); 10) Dechtiar et al.

1988 (ON); 11) Dechtiar \& Lawrie 1988

(ON); 12) Dechtiar \& Nepszy 1988 (ON)

* Posthodiplostomum minimum centrarchi

Hoffman, 1958

Site: liver

Host: Perca flavescens

Distribution: ON

Record: $\quad$ Molnar et al. 1974

*Posthodiplostomum minimum minimum

(MacCallum, 1921) Hoffman, 1958

Site: mesenteries

Hosts: Carpiodes cyprinus (1)

Catostomus commersoni (2)
Hybognathus hankinsoni $(2,3)$

Notropis atherinoides (1)

Notropis hudsonius (1)

Phoxinus eos (2)

Pimephales promelas $(2,3,4)$

Distribution: ON

Records: 1) Dechtiar 1972b; 2) Kakonge 1972; 3) Molnar et al. 1974; 4) Chan 1980

*Uvulifer ambloplitis (Hughes, 1927)

Dubois, 1938

Sites: fins, gills, musculature, subcutaneous

Hosts: Catostomus commersoni (6)

Culaea inconstans (5)

Esox lucius $(2,3,4,7,8,9)$

Etheostoma exile (6)

Hybognathus hankinsoni $(1,6)$

Margariscus margarita (1)

Notropis hudsonius (8)

Perca flavescens $(1,5,6)$

Phoxinus eos (1)

Phoxinus neogaeus (1)

Pimephales promelas $(1,2,6)$

Stizostedion vitreum (7)

Distribution: ON

Records: 1) Bangham 1941; 2)

Crossman 1962; 3) Dechtiar 1972a; 4)

Dechtiar 1972b; 5) Kakonge 1972; 6)

Molnar et al. 1974; 7) Dechtiar \& Christie 1988; 8) Dechtiar et al. 1988; 9) Dechtiar et al. 1988; 9) Dechtiar \& Nepszy 1988

\section{SUBFAMILY DIPLOSTOMINAE}

*Diplostomum adamsi Lester and Huizinga, 1977 
Site: eye

Host: Perca flavescens

Distribution: $\quad$ AB, BC, ON

Records: Tedla \& Fernando 1969d

(ON); Tedla \& Fernando 1972 (ON);

Lester 1977 (BC); Lester \& Huizinga

1977 (ON); Zelmer 1994 (AB - Carburn

Pond, Fincastle Reservoir, Garner

Lake, Pigeon Lake, Rattlesnake

Reservoir, Sherbourne Reservoir);

Zelmer \& Arai 1998 (AB - Garner

Lake)

*Diplostomum baeri bucculentum Dubois and Rausch, 1948

Site: eye

Hosts: Coregonus clupeaformis $(1,3,4)$

Couesius plumbeus (2)

Lota lota (2)

Oncorhynchus mykiss $(2,3,4)$

Prosopium williamsoni $(2,3,4)$

Ptychocheilus oregonensis (2)

Salvelinus malma $(2,3,4)$

Salvelinus namaycush $(3,4)$

Distribution: $\quad \mathrm{BC}, \mathrm{MB}$

Records: 1) Dick \& Rosen 1981 (MB); 2) Anonymous 1984 (BC); 3) Ching 1984a (BC); 4) Ching 1985 (BC)

*Diplostomum scudderi (Olivier, 1941)

Dubois, 1966

Synonym: Diplostomum baeri eucaliae Hoffman and Hundley, 1966

Sites: central nervous system, eye

Hosts: Culaea inconstans $(1,2,4)$

Gasterosteus aculeatus (3)

Distribution: $\quad \mathrm{BC}, \mathrm{ON}$

Records: $\quad$ 1) Dechtiar 1972a (ON);
2) Kakonge 1972 (ON); 3) Lester 1975

(BC); 4) Dechtiar et al. 1988 (ON)

*Diplostomum spathaceum (Rudolphi, 1819) Olsson, 1876

Synonym: Diplostomulum spathaceum

(Rudolphi, 1819) Hughes, 1929

Site: eye

Hosts: Acipenser fulvescens $(24,26)$

Carpiodes cyprinus $(25,27)$

Catostomus catostomus $(3,10,25$, 26, 28)

Catostomus commersoni $(3,5,7$,

$13,16,24,25,26,27)$

Coregonus artedii $(14,15,16,24$,

$25,25,28,29)$

Coregonus clupeaformis $(4,7,9$,

$10,14,15,20,22,24,25$ )

Cottus cognatus (10)

Cottus ricei (25)

Conesius plumbeus $(17,23,24$,

25)

Culaea inconstans $(25,29)$

Esox lucius $(10,24,25)$

Etheostoma exile $(5,24)$

Gasterosteus aculeatus $(2,8,24)$

Hybognathus hankinsoni (5)

Lota lota $(10,17,25,26)$

Nutropis atherinoides $(24,25)$

Notropis hudsonius $(24,25,26)$

Oncorhynchus kisutch $(25,26)$

Oncorhynchus mykiss $(17,24$,

$25,26)$

25, 26, 27)

Perca flavescens $(5,7,12,13$, 25, 26, 29)

Percopsis omiscomaycus $(7,24$,

Phoxinus neogaeus (25)

Pimephales promelas $(5,16,24$,

29) 
25, 26)

Prosopium cylindraceum $(4,10$,

Prosopium williamsoni (17)

Ptychocheilus oregonensis (17)

Pungitius pungitius $(1,9,19,20$,

Rhinichthys cataractae (25)

Salvelinus fontinalis $(4,6,11,18$, $21,24,25,29,30$ )

Salvelimus fontinalis $\mathrm{X}$ Salvelinus namaycush $(7,25,29)$

Salvelinus malma (17)

Salvelinus namaycush $(4,9,10$, $13,20,24,25)$

Stizostedion vitreum $(24,25,27)$

Thymallus arcticus (10)

Distribution: $\quad \mathbf{A B}, \mathrm{BC}, \mathrm{CA}, \mathrm{LB}, \mathrm{MB}, \mathrm{NF}$, NS, NT, ON, QC, YT

Records: 1) Hanek \& Threlfall 1970b (NF); 2) Hanek \& Threlfall 1970a (NF, LB); 3) Threlfall \& Hanek 1970a (LB); 4) Hicks \& Threlfall 1973 (LB); 5) Molnar et al. 1974 (ON); 6) Hare \& Frantsi 1974 (NS); 7) Collins \& Dechtiar 1974 (ON);

8) Lester 1975 (BC); 9) Leong 1975 (AB-Cold Lake); 10) Arthur et al. 1976 (YT); 11) Bell \& Margolis 1976 (CA); 12) Lester \& Lee 1976 (EXP); 13) Lester \& Huizinga 1977 (ON); 14) Watson 1977 (MB); 15) Watson \& Dick 1979 (MB); 16) Chan 1980 (ON); 17) Anonymous 1981 (BC); 18) Black 1981 (QC); 19) Curtis 1981(NT); 20) Leong \& Holmes 1981 (AB - Cold Lake); 21) Brassard et al. 1982 (QC); 22) Drouin 1982 (AB McGregor Lake, Travers Reservoir); 23) Anonymous 1984 (BC); 24) Dechtiar \& Christie 1988 (ON); 25) Dechtiar et al 1988 (ON); 26) Dechtiar \& Lawrie 1988 (ON); 27) Dechtiar \& Nepszy 1988 (ON); 28) Curtis 1988 (CA); 29) Dechtiar et al. 1989 (ON); 30) Wright 1989 (QC)
*Diplostomum spathaceum huronense (La Rue, 1927) Hughes, 1929

Synonyms: Diplostomum huronense (La Rue, 1927) Hughes and Hall, 1929; Diplostomulum huronense (La Rue, 1927) Hughes and Hall, 1929

Site: eye

Host: Perca flavescens

Distribution: ON

Records: Tedla \& Fernando 1969a:

Tedla 1969; Tedla \& Fernando 1972;

Dechtiar \& Christie 1988

* Diplostomum spathaceum indistinctum (Guberlet, 1923) Hughes, 1929

Synonyms: Diplostomum flexicaudum (Cort and Brooks, 1928); Diplostomulum flexicaudum (Cort and Brooks, 1928) Hunter, 1942

Site: eye

Hosts: Catostomus catostomus $(1,6)$ Catostomus commersoni $(1,2,3,4$,

6)

Coregonus artedii $(5,6)$

Coregonus clupeaformis $(4,5,6,7)$

Lota lota (2)

Moxostoma anisurum (3)

Moxostoma macrolepidotum (4)

Notropis atherinoides (4)

Perca flovescens (6)

Percopsis omiscomaycus (4)

Salvelinus fontinalis $\mathrm{X}$ Salvelinus namaycush (6)

Distribution: $\quad$ AB, MB, ON

Records: 1) Bangham 1955 (ON); 2)

Fillion 1961 (AB-Cold Lake, Lac Ste.

Anne); 3) Dechtiar 1972a (ON); 4)

Dechtiar 1972b (ON); 5) Watson 1977

(MB); 6) Dechtiar \& Berst 1978 (ON); 7) 
Dick \& Rosen 1981 (MB)

*Diplostomum sp.

Includes: Diplostomulum sp.

Sites: central nervous system, digestive tract, eye, musculature

Hosts: Acipenser fulvescens $(3,5,26,30$,

32.)

Carpiodes cyprinus $(21,30,32)$

Catostomus catostomus $(2,4,8$,

11,15)

Catcstomus commersoni $(2,11$,

$31,32)$

Catostomus macrocheilus $(2,8$,

15)

Coregonus artedii $(3,5)$

Coregonus clupeaformis $(3,5,8$,

$9,10,11,12,14,15,16,17,18,25)$

Cottus cognatus $(8,15)$

Cottus ricei (23)

Couesius plumbeus $(2,3,5,11)$

Culaea inconstans (3)

Esox lucius (3, 5, 11, 12, 31, 32)

Etheostoma exile (5)

Gasterosteus aculeatus (2. 3, 11,

15)

Lota lota $(2,5,15,16)$

Margariscus margarita (11)

Moxostoma anisurum $(31,32)$

Myoxocephalus thompsoni (13)

Notropis atherinoides $(5,31,32)$

Notropis hudsonius $(3,5,31,32)$

Oncorhynchus clarki (2)

Oncorhynchus kisutch $(2,16)$

Oncorhynchus mykiss $(2,3,8$,

$15,16,24)$

Perca flavescens $(3,5,12,17$,

$18,30,31,32$ )

Percina caprodes $(1,5,32)$

Percopsis omiscomaycus (3, 5,
$31,32)$

Phoxinus eos (3)

Pimephales promelas $(5,22)$

Prosopium cylindraceum (2, 3,

11)

Prosopium williamsoni $(2,8,15$,

16)

$15,16)$

Ptychocheilus oregonensis (2, 8,

Rhinichthys cataractae $(5,32)$

Richardsonius balteatus $(2,8,15$,

16)

Salvelinus fontinalis $(3,7,11,19$, 20, 29, 33)

Salvelinus malma $(8,15)$

Salvelinus namaycush $(3,5,8$,

$11,15,16)$

Stizostedion canadense $(3,5)$

Stizostedion vitreum $(3,5,17)$

Thymallus arcticus (8)

Distribution: $\quad$ AB, BC, LB, MB, NB, NF, NS, ON, QC, SK

Records: 1) Bangham \& Hunter 1939

(ON); 2) Bangham \& Adams 1954 (BC);

3) Bangham 1955 (ON); 4) Threlfall \&

Hanek 1970b (LB); 5) Dechtiar 1972a

(ON); 6) Scott \& Crossman 1973 (ON);

7) Frantsi et al. 1975 (NS); 8)

Anonymous 1978 (BC); 9) Pybus \&

Samuel 1978 (AB - Blue Lake, Cache

Lake, Ja.ivis Lake); 10) Rau et al 1979

(QC); 11) Curtis \& Rau1980 (QC); 12)

Samuel 1981 (AB - Jarvis Lake (E.

lucius, P. flavescens), Blue Lake, Cache

Lake, Jarvis Lake (C. clupeaformis));

13) Black \& Lankester 1981a (ON); 14)

Drouin 1982 (AB - McGregor Lake,

Travers Reservoir); 15) Arai \& Mudry 1983 (BC); 16) Anonymous 1984 (BC);

17) Poole \& Dick 1985 (MB); 18)

Samuel 1985 (AB - Blue Lake, Cache

Lake, Jarvis Lake); 19) Frimeth 1987a 
(NB); 20) Frimeth 1987b (NB); 21) Szalai \& Dick 1987a (MB); 22) Dechtiar \& Christie 1988 (ON); 23) Dechtiar et al. 1988 (ON); 24) Szalai \& Dick 1988 (MB); 25) Stock 1988 (AB - Jarvis Lake); 26) Choudhury et al. 1990 (MB); 27) Albert \& Curtis 1991 (QC); 28)

Marcogliese \& Cone 1991a (LB, NB, NF, NS, QC); 29) Marcogliese \& Cone 1991b (NF); 30) Szalai \& Dick 1991a (MB); 31) Szalai et al. 1992 (MB); 32) Choudhury \& Dick 1993 (MB, SK); 33) Dubois et al. 1996 (QC)

*Tylodelphys scheuringi (Hughes, 1929) Dubois, 1938

Synonym: Diplostomum scheuringi Hughes, 1929

Site: eye

Hosts: Catostomus macrocheilus $(8,9)$ Coregonus clupeaformis (9)

Lota lota $(2,8,9)$

Oncorhynchus mykiss $(8,9,11)$

Perca flavescens $(1,3,4,5,6,7$,

13)

Percina caprodes $(6,10)$

Percopsis omiscomaycus (12)

Prosopium williamsoni $(8,9)$

Ptychocheilus oregonensis (9)

Rhinichthys cataractae (6)

Richardsonius balteatus (9)

Stizostedion vitreum (6)

Distribution: $\quad \mathbf{A B}, \mathrm{BC}, \mathrm{MB}, \mathrm{ON}$

Records: 1) Bangham \& Hunter 1939

(ON); 2) Fillion 1961 (AB - Lac Ste

Anne); 3) Tedla \& Fernando 1969a (ON);

4) Tedla 1969 (ON); 5) Tedla \& Fernando 1972 (ON); 6) Dechtiar 1972a (ON); 7)

Molnar et al. 1974 (ON); 8) Anonymous

1981 (BC); 9) Anonymous 1984 (BC);
10) Dechtiar \& Christie 1988 (ON); 11) Szalai \& Dick 1988 (MB); 12) Dechtiar et al. 1989 (ON); 13) Zelmer 1994 (AB Beta Lake, Burntstick Lake, Carburn Pond, Coal Lake, Elkwater Lake, Fincastle Lake, Garner Lake, Hastings Lake, Joker Lake, Pigeon Lake, Pine Lake, Rattlesnake Lake, Sherburne Lake, Spruce Coulee Reservoir, Sylvan Lake); 14) Zelmer \& Arai 1998 (AB Garner Lake)

\section{FAMILY STRIGEIDAE}

*Apatemon gracilis (Rudolphi, 1819) Szidat, 1928

Synonym: Apatemon gracilis pellucidus (Yamaguti, 1933) Dubois, 1953

Sites: central nervous system, coelom, eye, gills, musculature

Hosts: $\quad$ Culaea inconstans $(3,4,6,7)$ Gasterosteus aculeatus (1) Perca flavescens (8) Pungitius pungitius $(2,5)$

Distribution: $\mathbf{A B}, \mathbf{B C}, \mathbf{Q C}$ Records: 1) Lester 1975 (BC); 2) Leong 1975 (AB - Cold Lake); 3) Rau \& Gordon 1977 (QC); 4) Rau \& Gordon 1978 (QC); 5) Leong \& Holmes 1981 (AB - Cold Lake); 6) Gordon \& Rau 1982a (QC); 7) Gordon \& Rau 1982b (QC); 8) Zelmer 1994 (AB - Rattlesnake Reservoir, Shelbourne Reservoir)

*Ichthyocotylurus erraticus (Rudolphi, 1809) Niewiadomska, 1971

Synonyms: Cotylurus erraticus (Rudophi, 1809) Szidat, 1928; 
1928; Tetracotyle intermedia

Hughes, 1928

Sites: mesenteries, pericardium

Hosts: Catostomus commersoni (2)

Coregonus artedii $(1,4,5,6,7,8$, $10,11,12,13)$

Coregonus clupeaformis $(1,2,3$,

$4,7,8,9,10,11,12,13,14)$

Oncorhynchus mykiss (12)

Perca flavescens (2)

Percopsis omiscomaycus $(2,11)$

Prosopium cylindraceum $(12,13)$

Pungitius pungitius $(4,8)$

Salvelinus fontinalis (13)

Salvelinus fontinalis $\mathrm{X}$

11)

Salvelinus namaycush $(2,6$,

Distribution: $\quad \mathbf{A B}, \mathrm{MB}, \mathrm{ON}$

Records: 1) Dechtiar 1972a (ON); 2)

Collins \& Dechtiar 1974 (ON); 3) Leong

\& Holmes 1974a (AB - Cold Lake); 4)

Leong 1975 (AB - Cold Lake); 5)

Watson 1977 (MB); 6) Dechtiar \& Berst 1978 (ON); 7) Watson \& Dick 1979

(MB); 8) Leong \& Holmes 1981 (AB -

Cold Lake); 9) Pybus \& Samuel 1981

(AB - Wabamun Lake); 10) Dechtiar \&

Christie 1988 (ON); 11) Dechtiar et al.

1988 (ON); 12) Dechtiar \& Lawrie 1988

(ON) 13) Dechtiar \& Nepszy 1988 (ON);

14) Dechtiar et al. 1989 (ON)

*Ichthyocotylurus pileatus (Rudolphi, 1802)

Niewiadomska, 1971

Synonym: Tetracotyle diminuta Hughes, 1928

Site: mesenteries

Hosts: Perca flavescens $(2,3,4,5)$

Percopsis omiscomaycus $(1,3)$

Stizostedion vitreum (4)
Distribution: ON

Records: 1) Bangham \& Hunter $1939 ; 2)$

Tedla \& Fernando 1972; 3) Dechtiar \&

Christie 1988; 4) Dechtiar \& Lawrie

1988; 5) Dechtiar \& Nepszy 1988

*Ichthyocotylurus platycephalus (Creplin, 1825) Niewiadomska, 1971

Synonym: Cotylurus communis

(Hughes, 1928) La Rue, 1932

Sites: liver, mesenteries, pericardium

Hosts: Catostomus catostomus $(2,3,4)$

Catostomus commersoni (2)

Catostomus macrocheilus $(3,4)$

Oncorhynchus kisutch (3)

Percopsis omiscomaycus (2)

Phoxinius neogaeus $(1,2)$

Prosopium williamsoni $(3,4)$

Ptychocheilus oregonensis $(3,4)$

Stizostedion canadense (2)

Stizostedion vitreum (2)

Distribution: $\mathrm{BC}, \mathrm{ON}$

Records: 1) Bangham \& Hunter 1939

(ON); 2) Dechtiar 1972a (ON); 3)

Anonymous 1981 (BC); 4) Anonymous

1984 (BC)

* Tetracotyle sp.

Synonyms: Diplostomum parvula Stafford, 1904; Tetracotyle

parvula (Stafford, 1904) Mataré, 1909

Sites: heart, kidneys, mesenteries, musculature, pericardium

Hosts: Carpiodes cyprinus (27)

29)

Catostomus catostomus $(3,13,18$,

Catostomus commersoni $(2,11$, 
$13,16,18,26,27,29,33)$

Catostomus macrocheilus $(3,13$,

17)

Coregonus artedii (33)

Coregonus clupeaformis $(12,13$,

$17,18,22,27)$

Cottus cognatus $(13,18,24,29)$

Cottus ricei (25)

Couesius plumbeus $(3,24)$

Culaea inconstans $(3,8,10,25$,

29)

Esox lucius $(8,24,25,33)$

Etheostoma exile $(1,4,8,11,23)$

Gasterosteus aculeatus (3)

Hiodon tergisus (1)

Hybognathus hankinsoni (11)

Lota lota $(8,12,23,25,26)$

Margariscus margarita (29)

Moxostoma anisurum (33)

Notropis hudsonius $(1,4,8,24$,

$25,26)$

Oncorhynchus mykiss $(13,18,21$,

Perca flavescens $(4,7,8,14,15$, $16,18,31,32,33$ )

Percina caprodes (1)

Percopsis omiscomaycus $(4,26$, 29, 33)

Phoxinus eos (4)

Phoxinus neogaeus (29)

Prosopium cylindraceum $(12,25)$

Prosopium williamsoni $(3,13,18$,

21)

Pungitius pugitius (8)

Rhinichthys cataractae $(3,8,13$,

$30,34)$

Richardsonius balteatus $(3,13$,

Salvelinus fontinalis $(6,2), 24$,

Salvelinus fontinalis X Salvelinus namaycush (29)
Salvelinus malma $(3,13,18)$

Salvelinus namaycush $(12,13,18)$

Stizostedion canadense (9)

27)

Stizostedion vitreum $(5,14,24$,

Thymallus arcticus $(12,13,18)$

Distribution: $\quad$ AB, BC, MB, NF, ON, YT

Records: 1) Bangham \& Hunter 1939

(ON); 2) Bangham 1941 (ON); 3)

Bangham \& Adams 1954 (BC); 4)

Bangham 1955 (ON); 5) Ryder 1963

(AB - Iosegun Lake); 6) Sandeman \&

Pippy 1967 (NF); 7) Paetz \& Nelson

1970 (AB - unspecified); 8) Dechtiar

1972a (ON); 9) Dechtiar 1972b (ON);

10) Molnar et al. 1974 (ON); 11) Arthur et al 1076 (YT); 12) Anonymous 1978

(BC); 13) Anthony 1978b (ON); 14)

Pybus \& Samuel 1978 (AB - Cache

Lake); 15) Chan 1980 (ON); 16) Samuel

1981 AB - Cache Lake, Jarvis Lake,

(C. clupeaformis, $P$. flavescens), Blue

Lake, Gregg Lake, (C. clupeaformis)];

17) McCart et al. 1982 (AB - Lake

Athabasca, Athabasca River); 18) Arai

\& Mudry 1983 (BC); 19) Cone \& Ryan

1984 (NF); 20) Anonymous 1984 (BC);

21) Samuel 1985 [AB - Blue Lake, Gregg Lake ( $C$. clupeaformis), Cache Lake, Jarvis Lake, (C. clupeaformis, $P$. flavescens )]; 22) Anthony 1987 (ON);

23) Dechtiar \& Christie 1988 (ON); 24)

Dechtiar et al.1988 (ON); 25) Dechtiar \&

Lawrie 1988 (ON); 26) Dechtiar \&

Nepszy 1988 (ON); 27) Stock 1988

(AB- Jarvis Lake); 28) Dechtiar et al.

1989 (ON); 29) Marcogliese \& Cone

$1991 b$ (NF); 30) Szalai \& Dick 1991

(MB); 31) Nelson \& Paetz 1992 (AB unspecified); 32) Szalai et al. 1992 (MB); 33) Dubois et al. 1996 (QC) 
*Strigeidae gen. sp.

Sites: central nervous system, liver, mesenteries, musculature, subcutaneous

Hosts: Etheostoma exile (2)

Notropis atherinoides (2)

Oncorhynchus kisutch (7)

Perca flavescens $(3,4,6)$

Percina caprodes (2)

Prosopium williamsoni $(6,7)$

Ptychocheilus oregonensis $(5,7)$

Salvelinus fontinalis (1)

Distribution: $\quad \mathrm{BC}, \mathrm{MB}, \mathrm{NB}, \mathrm{ON}, \mathrm{QC}$

Records: 1) Ricker 1932 (ON); 2)

Bangham \& Hunter 1939 (ON); 3) Lyster 1940a (QC); 4) McLeod 1943 (MB); 5)

Anonymous 1981 (BC); 6) Pike \& Burt

1981 (NB); 7) Anonymous 1984 (BC)

SUPERFAMILY BRACHYLAEMOIDEA

\section{FAMILY BUCEPHALIDAE}

Bucephalus elegans Woodhead, 1929

Site: pyloric caeca

Host: Perca flavescens

Distribution: ON

Records: Bangham 1941; Bangham 1955

*Bucephalus sp.

Sites: digestive tract, coelom

Hosts: Catostomus catostomus (5)

Catostomus commersoni (6)

Hiodon alosoides (2)

Notropis hudsonius $(1,7)$

Stizostedion vitreum (6)
Distribution: $\quad \mathrm{MB}, \mathrm{ON}$

Records: 1) Bangham 1955 (ON); 2)

Kennedy \& Sprules 1967 (MB); 3)

Dechtiar 1972b (ON); 4) Dechtiar \&

Christie 1988 (ON); 5) Dechtiar \& Lawrie 1988 (ON); 6) Dechtiar \& Nepszy 1988

(ON); 7) Dechtiar et al. 1988 (ON)

Paurorhynchus hiodontis Dickerman, 1954

Sites: coelom, gills, heart

Hosts: Hiodon alosoides $(1,2,4)$

Hiodon tergisus (3)

Distribution: $\quad$ AB, MB, SK

Records: 1) Margolis 1964 (SK); 2)

Donald \& Kooyman 1974 (AB - Chenal des Quatre Fourches, Mamawi Lake);

3) Glenn 1980 (MB); 4) Szalai et al. 1992 (MB)

Prosorhynchus ozakii (Nagaty, 1937)

Margolis and Arthur, 1979

Synonym: Bucephaloides ozakii Nagaty, 1937

Site: intestine

Host: Salvelinus malma

Distribution: $\quad \mathrm{BC}$

Record: Bangham \& Adams 1954

Prosorhynchus pusilla (Stafford, 1904)

Margolis and Arthur, 1979

Synonyms: Gasterostomum pusillum

Stafford, 1904; Bucephalus pusilla

(Stafford, 1904); Bucephalopsis pusilla

(Stafford, 1904) Eckmann, 1932

Sites: intestine, pyloric caeca, stomach

Hosts: Perca flavescens (8) 
Stizostedion canadense $(4,7)$

Stizostedion vitreum $(1,2,3,4,5$, $6,8,9,10$ )

Distribution: $\mathrm{MB}, \mathrm{ON}, \mathrm{QC}$

Records: 1) Stafford 1904 (QC); 2)

Woodhead 1930 (ON); 3) Lyster 1939

(QC); 4) Bangham \& Hunter 1939 (ON);

5) Bangham 1955 (ON); 6) Dickson 1964

(MB); 7) Dechtiar 1972a (ON); 8) Poole

\& Dick 1985 (MB); 9) Dechtiar \&

Christie 1988 (ON); 10) Dechtiar et al.

1988 (ON)

*Rhipidocotyle papillosa (Woodhead, 1929)

Eckmann, 1932

Includes: Gasterostomum pusillum of Cooper (1915a)

Site: musculature

Host: Perca flavescens

Distribution: ON

Record: Cooper 1915a

Rhipidocotyle papillosa (Woodhead, 1929) Eckmann, 1932

Includes: Gasterosomum pusillum of Cooper (1915a)

Site: [digestive tract]

Hosts: Perca flavescens Stizostedion vitreum

Distribution: $\quad \mathrm{MB}$

Record: $\quad$ Poole \& Dick 1985

*Rhipidocotyle sp.

Site: $\quad$ subcutaneous (fins)

Host: Hybognathus hankinsoni

Distribution: ON
Record: $\quad$ Molnar et al. 1974 (ON)

ORDER HEMIURIFORMES

SUPERFAMILY HEMIUROIDEA

FAMILY AZYGIIDAE

Azygia angusticauda (Stafford, 1904)

Marter, 1926

Synonyms: Mimodistomum angusticaudum Stafford, 1904;

Ptychogonimus fontanus Lyster, 1939

Site: digestive tract

Hosts: Catostomus commersoni (17) Esox lucius $(12,15,16,19,21$,

24)

Etheostoma exile $(11,15,17)$

Lota lota $(1,15,20,23)$

Perca flavescens $(3,4,6,8,9,10$,

$11,13,14,18,22$ )

Salvelinus fontinalis (4)

Salvelinus namaycush (7)

Stizostedion vitreum $(1,2,5,14$,

$15,18,21,24)$

Distribution: ON, QC

Records: 1) Stafford 1904 (QC); 2)

Bangham \& Hunter 1939 (ON); 3) Lyster

1939 (QC); 4) Lyster 1940 (QC); 5)

Miller 1940b (QC); 6) Bangham 1941

(ON); 7) MacLulich 1943b (ON); 8)

Bangham \& Venard 1946 (ON); 9)

Fantham \& Porter 1948 (QC); 10)

Choquette 1951c (QC); 11) Bangham

1955 (ON); 12) Crossman 1962 (ON);

13) Tedla 1969 (ON); 14) Tedla \&

Fernando 1972 (ON); 15) Dechtiar 1972a 
(ON); 16) Dechtiar 1972b (ON); 17)

Molnar et al. 1974 (ON); 18) Anthony 1978b (ON); 19) Anthony 1983 (ON); 20) Anthony 1987 (ON); 21) Dechtiar \& Christie 1988 (ON); 22) Dechtiar et al. 1988 (ON); 23) Dechtiar \& Lawrie1988 (ON); 24) Dechtiar \& Nepszy 1988 (ON)

Azygia longa (Leidy, 1851) Stafford, 1904;

Synonyms: Megadistomum longa (Leidy, 1851) Stafford, 1904; Azygia acuminata Goldberger, 1911

Site: digestive tract

Hosts: Acipenser fulvescens (17)

Esox lucius (1, 2, 3, 4, 5, 7, 9, 13,

16)

Hiodon alosoides (16)

Lota lota $(1,10)$

Perca flavescens $(2,8,15,16)$

Salvelinus fontinalis $(6,11,12,14)$

Salvelinus namaycush (2)

Stizostedion vitreum (2)

Distribution: $\quad \mathrm{MB}, \mathrm{NB}, \mathrm{NF}, \mathrm{ON}, \mathrm{QC}$

Records: 1) Stafford 1904 (QC); 2)

Cooper 1915 (ON); 3) Lyster 1939 (QC);

4) Fantham \& Porter 1948 (QC); 5)

Bangham 1955 (ON); 6) Sandeman \&

Pippy 1967 (NF); 7) Dechtiar 1972a

(ON); 8) Anthony 1978b (ON); 9)

Anthony 1983 (ON); 10) Anthony 1987

(ON); 11) Frimeth 1987a (NB); 12)

Frimeth 1987b (NB); 13) Dechtiar et al.

1988 (ON); 14) Marcogliese \& Cone

$1991 b$ (NF); 15) Szalai \& Dick 1991

(MB); 16) Szalai et al.1992 (MB); 17)

Choudhury \& Dick 1993 (MB)

Azygia sp.
Site: not specified

Host: Stizostedion vitreum

Distribution: ON

Record: Anthony 1976

\section{SUBFAMILY LEUCERUTHRINAE}

*Leuceruthrus micropteri Marshall and Gilbert, 1905

Site: digestive tract

Hosts: Hiodon tergisus

Perca flavescens

Percina caprodes

Distribution: ON

Record: Bangham \& Hunter 1939

Leuceruthrus micropteri Marshall and

Gilbert, 1905

Site: digestive tract

Hosts: Notropis atherinoides

Notropis hudsonius

Distribution: ON

Record: Bangham \& Hunter 1939

SUPERFAMILY SCHISTOSOMATOIDEA

FAMILY SANGUINICOLIDAE

Sanguinicola occidentalis Van Cleave and Mueller, 1932

Site: circulatory system

Hosts: Perca flavescens $(1,7,8)$

Stizostedion vitreum $(1,2,3,4,5$, 
$6,7)$

Distribution: ON

Records: 1) Dechtiar 1972b; 2) Anthony 1976; 3) Anthony 1978b; 4) Dechtiar \& Christie 1988; 5) Dechtiar et al. 1988; 6) Dechtiar \& Lawrie 1988; 7) Dechtiar \& Nepszy 1988; 8) Dechtiar et al. 1989

Sanguinicola sp.

Site: circulatory system

Hosts: Carpiodes cyprinus $(2,5)$

Catostomus catostomus $(3,4)$

Catostomus commersoni $(3,5,6)$

Moxostoma anisurum (1)

Moxostoma macrolepidotum (2)

Notropis hudsonius $(2,4)$

Pimephales promelas (1)

Distribution: ON

Record: 1) Dechtiar 1972a; 2) Dechtiar 1972b; 3) Dechtiar et al. 1988; 4)

Dechtiar \& Lawrie 1988; 5) Dechtiar \&

Nepszy 1988; 6) Dechtiar et al. 1989

Sanguinicolidae gen. sp.

Site: circulatory system

Host: Catostomus catostomus

Distribution: $\quad \mathrm{BC}$

Records: Anonymous 1981; Anonymous 1984

ORDER PLAGIORCHIIFORMES

SUBORDER ALLOCREADIATA

SUPERFAMILY ALLOCREADIOIDEA
FAMILY ALLOCREADIIDAE

Allocreadium lobatum Wallin, 1909

Synonyms: Distomum isoporum (Looss, 1894) Odhner, 1901 = Distomum isoporum Looss, 1894; Allocreadium isoporum (Looss, 1894) Odhner, 1901

Site: intestine

Hosts: Catostomus catostomus $(1,5)$

Catostomus commersoni $(4,10)$

Couesius plumbeus $(1,3)$

Esor lucius (6)

Notropis hudsonius $(2,12)$

Oncorhynchus mykiss (1)

Phoxinus eos $(8,13)$

Pimephales promelas (4)

Prosopium williamsoni (1)

Pychocheilus oregonensis $(1,7,8$, 9)

Rhinichthys cataractae (1)

Thymallus arcticus (6)

Distribution: $\quad$ BC, LB, NB, ON, QC, YT Records: 1) Bangham \& Adams 1954

(BC); 2) Bangham 1955 (ON); 3)

Threlfall \& Hanek 1971 (LB); 4) Dechtiar 1972a (ON); 5) Hanek \& Molnar 1974 (QC); 6) Arthur et al. 1976 (YT); 7) Anonymous 1978 (BC); 8) Arai \& Mudry 1983 (BC); 9) Anonymous 1984 (BC); 10) Courtney 1987 (ON); 11) Dechtiar et al. 1988 (ON); 12) Dechtiar \& Lawrie 1988 (ON); 13) Dubois et al. 1996 (QC)

Allocreadium sp.

Site: intestine

Host: Etheostoma exile

Distribution: ON

Record: Bangham \& Hunter 1939 
Bunodera eucaliae (Miller, 1936) Miller, 1936

Synonym: Bunoderina eucaliae Miller, 1936

Site: intestine

Hosts: Culaea inconstans $(1,2,3,4,5,6$, $7,8,9,10,11,12$ )

Gasterosteus aculeatus $(4,8)$

Pungitius pungitius (6)

Distribution: $\quad \mathrm{BC}, \mathrm{ON}, \mathrm{QC}$

Records: 1) Miller 1936 (QC); 2)

Bangham \& Hunter 1939 (ON); 3) Miller 1940b (QC); 4) Bangham \& Adams 1954 (BC); 5) Bangham 1955 (ON); 6)

Dechtiar 1972a (ON); 7) Kakonge 1972 (ON); 8) Dechtiar \& Christie 1988 (ON);

9) Dechtiar et al. 1988 (ON); 10) Dechtiar et al. 1989 (ON); 11) Caira 1989 (BC, ON, QC); 12) Brooks 1992 (ON)

Bunodera luciopercae (Müller, 1776) Lühe, 1909

Synonym: Distomum nodulosum (Frölich, 1791)

Site: intestine

Hosts: Acipenser fulvescens (1)

Esox lucius $(8,12)$

Gasterosteus aculeatus (11)

Oncorhynchus mykiss $(19,20,22)$

Perca flavescens $(3,4,6,7,9,13$, $14,15,16,23$ )

Prosopium cylindraceum (17)

Pungitius pungitius $(10,18,21)$

Salvelinus malma (18)

Salvelinus namaycush (17)

Distribution: $\quad$ AB, BC, LB, NF, ON, QC

Records: 1) MacCallum 1895 (ON); 2)

Stafford 1902 (QC); 3) Bangham \&

Hunter 1939 (ON); 4) Fantham \& Porter
1948 (QC); 5) Sandeman \& Pippy 1967 (NF); 6) Tedla \& Fernando 1969a (ON); 7) Tedla 1969 (ON); 8) Threlfall \& Hanek 1970b (LB); 9) Tedla \& Fernando 1972 (ON); 10) Hanek \& Threlfall 1970b (NF); 11) Hanek \& Threlfall 1970a (LB, NF); 12) Threlfall \& Hanek $1970 \mathrm{~b}$ (LB); 13) Cannon 1970 (ON); 14) Cannon 1971 (ON); 15) Cannon 1972 (ON); 16) Cannon 1973 (ON); 17) Hicks \& Threlfall 1973 (LB); 18) Leong 1975 (AB - Cold Lake); 19) Mudry \& Anderson [AB Amethyst Lake ( $O$. mykiss, $S$. fontinalis), Moat Lake (O. mykiss), BC ( $S$. fontinalis, $S$. malma, $S$. namaycush)]; 20) Anonymous 1978 (BC); 21) Leong \& Holmes 1981 (AB - Cold Lake); 22) Arai \& Mudry 1983 (BC); 23) Caira 1989 (AB - unspecified; NF, ON, QC); 24) Zelmer 1994 (AB - Beta Lake, Burntstick Lake, Carburn Pond, Coal Lake, Elkwater Lake, Fincastle Lake, Joker Lake, Pigeon Lake, Pine Lake, Rattlesnake Reservoir, Sherbourne Reservoir, Spruce Coulee Reservoir, Sylvan Lake); 25) Zelmer \& Arai 1998 (AB - Garner Lake)

Bunodera mediovitellata Tsimbalyuk and Roytman in Skrjabin and Koval, 1966

Synonym: Allobunodera mediovitellata (Tsimbalyuk and Roytman in Skrjabin and Koval, 1966) Yamaguti, 1971

Site: intestine

Host: Gasterosteus aculeatus

Distribution: $\quad \mathrm{BC}$

Records: Lester 1975; Kennedy 1979; Caira 1989 
Bunodera sacculata Van Cleave and Mueller, 1932

Synonyms: Allobunodera sacculata

(Van Cleave and Mueller, 1932)

Yamaguti, 1971; Bunoderina sacculata

(Van Cleave and Mueller, 1932)

Yamaguti, 1958

Site: intestine

Hosts: Lota lota (10)

Perca flavescens $(1,2,3,4,5,6,7$,

$8,9,10,11,12,13,14,15,16,17)$

Stizostedion vitreum $(11,13)$

Distribution: $\quad \mathrm{MB}, \mathrm{ON}, \mathrm{QC}$

Records: 1) Lyster 1939 (QC); 2)

Bangham 1941 (ON); 3) Bangham \&

Venard 1946 (ON); 4) Bangham 1955

(ON); 5) Tedla \& Fernando 1969a (ON);

6) Tedla 1969 (ON); 7) Cannon 1970

(ON); 8) Cannon 1971 (ON); 9) Tedla \&

Fernando 1972 (ON); 10) Cannon 1972

(ON); 11) Dechtiar 1972a (ON); 12)

Cannon 1973 (ON); 13) Poole \& Dick

1985 (MB); 14) Dechtiar \& Christie 1988

(ON); 15) Dechtiar et al. 1988 (ON); 16)

Dechtiar \& Nepszy 1988 (ON); 17) Caira

1989 (ON)

Bunodera sp.

Site: [digestive tract]

Host: Perca flavescens

Distribution: QC

Record: Fantham \& Porter 1948

Crepidostomum auriculatum (Wedl, 1858) Pratt, 1902

Synonym: Crepidostomum lintoni (Pratt in Linton, 1901) Hopkins, 1933
Site: intestine

Hosts: Acipenser fulvescens $(1,2,4,5,6$, $7,8,9,10,11,12,14,15,16)$ Coregonus clupeaformis (13) Perca flavescens (3)

Distribution: $\quad$ AB, MB, ON, QC, SK

Records: 1) Stafford 1904 (QC); 2) Cooper 1915 (ON); 3) Hopkins 1934 (ON); 4) Lyster 1939 (QC); 5) Bangham \& Hunter 1939 (ON); 6) Bangham 1955 (ON); 7) Dechtiar 1972a (ON); 8) Dechtiar 1972b (ON); 9) Anthony 1974 (ON); 10) Dechtiar \& Christie 1988 (ON); 11) Dechtiar et al. 1988 (ON); 12) Dechtiar \& Lawrie 1988 (ON); 13) Stock 1988 (AB - Jarvis Lake); 14) Choudhury et al. 1990 (MB, SK); 15) Swanson et al. 1991 (MB); 16) Choudhury \& Dick 1993 (MB, SK)

Crepidostomum cooperi Hopkins, 1931

Synonyms: Crepidostomum ambloplitis Hopkins, 1931; Crepidostomum fausti Hunninen and Hunter, 1933

Includes: Crepidostomum laureatum of Stafford (1904) and of Cooper (1915); Bunodera nodulosa of Stafford (1904) (partim); ?Crepidostomum cornutum of Cooper (1915) (partim)

Sites: gall bladder, intestine, pyloric caeca

Hosts: Catostomus commersoni $(14,27)$

Coregonus artedii (31)

Coregonus clupeaformis (11, 13, $33,34)$

Etheostoma exile (3)

Gasterosteus aculeatus (20)

Perca flavescens $(2,3,4,5,8,9$, $11,13,14,16,18,19,21,22,23,24,26$, $28,29,32,36,37,38,39,40,41,42$ ) 
Percopsis omiscomaycus (42)

Prosopium cylindraceum (11)

Salvelinus fontinalis $(1,2,6,7$,

$10,12,14,15,17,25,30,35$ )

Salvelinus namaycush (33)

Stizostedion canadense (42)

Stizostedion vitreum (42)

Distribution: $\quad \mathbf{A B}, \mathrm{LB}, \mathrm{MB}, \mathrm{NF}, \mathrm{NS}, \mathrm{ON}$, PE, QC

Records: 1) Stafford 1902 (QC); 2)

Stafford 1904 (QC); 3) Cooper 1915

(ON); 4) Hopkins 1931 (ON); 5) Hopkins

1934 (ON); 6) Richardson 1936 (QC); 7)

Richardson 1937 (QC); 8) Bangham \&

Hunter 1939 (ON); 9) Lyster 1939 (QC);

10) Lyster 1940a (QC); 11) Bangham

1941 (ON); 12) Richardson 1942 (QC);

13) Bangham \& Venard 1946 (ON); 14)

Fantham \& Porter 1948 (QC); 15)

Choquette 1948a (QC); 16) Stewart-Hay 1951 (MB); 17) Choquette 1954 (QC);

18) Bangham 1955 (ON); 19) Dickson

1964 (MB); 20) Hanek \& Threlfall 1969c

(NF); 21) Tedla \& Fernando 1969a (ON);

22) Tedla 1969 (ON); 23) Hanek \&

Threlfall 1969c (NF); 24) Cannon 1970

(ON); 25) Threlfall \& Hanek 1970c (NF);

26) Tedla \& Fernando 1972 (ON); 27)

Dechtiar 1972a (ON); 28) Cannon 1972

(ON); 29) Cannon 1973 (ON); 30) Hanek

\& Molnar 1974 (QC); 31) Anthony 1978a

(ON); 32) Anthony 1978b (ON); 33)

Chinniah \& Threlfall 1978 (LB); 34)

Pybus \& Samuel 1978 (AB - Blue Lake,

Gregg Lake, Jarvis Lake); 35) Black

1981a (QC); 36) Poole \& Dick 1985

(MB); 37) Dechtiar \& Christie 1988

(ON); 38) Dechtiar et al. 1988 (ON); 39)

Dechtiar \& Nepszy 1988 (ON); 40) Caira

1989 (MB, NB, NF, NS, ON, PE, QC);

41) Szalai \& Dick 1991a (MB); 42) Szalai

et al. 1992 (MB)
Crepidostomum cormutum (Osborn, 1903)

Stafford, 1904

Sites: gall bladder, intestine, pyloric caeca

Hosts: Perca flavescens (2)

Salvelinus fontinalis (1)

Distribution: NS, ON, QC

Records: 1) Fantham \& Porter 1948

(NS, QC); 2) Dechtiar \& Lawrie 1988 $(\mathrm{ON})$

Crepidostomum farionis (Müller, 1780)

Lühe, 1909

Sites: gall bladder, intestine, pyloric caeca

Hosts: Catostomus catostomus $(28,35)$

Catostomus commersoni (51)

Coregonus artedii $(15,16,19,22$,

$26,29,41,43$ )

Coregonus clupeaformis $(3,12$,

$14,15,16,17,18,19,21,23,26,29,33$, 41)

Cottus cognatus $(21,33)$

Esox lucius $(19,27)$

Gasterosteus aculeatus $(10,16)$

Lota lota $(7,18,21,33,34)$

Oncorhynchus clarki $(7,18,30)$

Oncorhynchus kisutch $(7,15,16$,

$28,29,30,35)$

Oncorhynchus mykiss $(4,6,7,9$,

$18,21,28,33,35,42$ )

Perca flavescens $(14,37)$

Prosopium cylindraceum $(1,3$,

$5,7,8,17,41,45)$

Prosopium williamsoni $(7,18$,

$21,28,33,35,50)$

Ptychocheilus oregonensis (35)

Salmo trutta $(9,11)$

Salvelinus fontinalis $(2,3,4,5$, 
$7,9,11,12,13,18,23,24,25,36,38$, $39,40,41,44,47,48,49)$

Salvelinus malma $(7,21,28,33$,

35)

$41,45)$

Salvelinus namaycush $(2,3,12$, $15,17,18,23,31,32,34,35$,

Thymallus arcticus $(7,17,21$,

$33,45)$

Distribution: $\quad \mathbf{A B}, \mathrm{BC}, \mathrm{CA}, \mathrm{LB}, \mathrm{MB}, \mathrm{NB}$, NF, NS, NT, ON, PE, QC, YT

Records: 1) Bangham 1941 (ON); 2)

MacLulich 1943b (ON); 3) Bangham \&

Venard 1946 (ON); 4) Fantham \& Porter 1948 (QC); 5) Choquette 1948a (QC); 6)

Rawson 1953 (AB - Amethyst Lake); 7)

Bangham \& Adams 1954 (BC); 8)

Bangham 1955 (ON); 9) Sandeman \&

Pippy 1967 (NF); 10) Threlfall 1968

(NF); 11) Threlfall \& Hanek 1970c (NF);

12) Hicks \& Threlfall 1973 (LB); 13)

Hare \& Frantsi 1974 (NS); 14) Collins \&

Dechtiar 1974 (ON); 15) Leong \&

Holmes 1974a (AB - Cold Lake); 16)

Leong 1975 (AB - Cold Lake); 17)

Arthur et al. 1976 (YT); 18) Mudry \&

Anderson 1977 [AB - Pyramid Lake

(O. mykiss, $S$. namaycush, $P$.

williamsoni), Moraine Lake, (O. mykiss,

$S$. namaycush), Lower Waterton Lake,

Linnet Lake, (C. clupeaformis), Upper

Waterton Lake (L. lota), Edith Lake,

Horseshoe Lake, Moat Lake ( $O$.

mykiss), Patricia Lake, Herbert Lake,

Snowflake Lake, (O. mykiss, $S$.

fontinalis), Mud Lake, Little Baker

Lake, Temple Lake, Upper

Consolation Lake, Vista Lake, Upper

Twin Lake, Crandell Lake ( $S$.

fontinalis), Boom Lake, Lower Altrude

Lake, Peyto Lake, (O. clarki), Lower

Consolation Lake, Babel Creek) ( $O$. clarki, S. fontinalis), BC (O. clarki, O. mykiss, S. fontinalis, S. malma, S. namaycush); 19) Watson 1977 (MB); 20) Dechtiar \& Berst 1978 (ON); 21) Anonymous 1978 (BC); 22) Anthony 1978a (ON); 23) Chinniah \& Threlfall 1978 (LB); 24) Gordon et al. 1978 (QC); 25) Thompson \& Threlfall $1978(\mathrm{QC})$; 26) Watson \& Dick 1979 (MB); 27) Watson \& Dick 1980 (MB); 28) Anonymous 1981 (BC); 29) Leong \& Holmes 1981 (AB - Cold Lake); 30) Margolis \& Moravec 1982 (BC); 31) Stewart \& Bernier 1982 (NT); 32) Stewart \& Bernier 1983 (NT); 33) Arai \& Mudry 1983 (BC); 34) Stewart \& Bernier 1984 (NT); 35) Anonymous 1984 (BC); 36) Cone \& Ryan 1984 (NF); 37) Poole \& Dick 1985 (MB); 38) Frimeth 1987a (NB); 39) Frimeth 1987b (NB); 40) Dechtiar et al. 1988 (ON); 41) Dechtiar \& Lawrie 1988 (ON); 42) Szalai \& Dick 1988 (MB); 43) Curtis 1988 (CA); 44) Baggs \& McT. Cowan 1989 (NF); 45) Caira 1989 (AB - unspecified; BC, MB, NB, NF, NS, NT, ON, PE, QC); 46) Dechtiar et al. 1989 (ON); 47) Wright et al.1989 (ON); 48) Albert \& Curtis 1991 (QC); 49) Marcogliese \& Cone 1991b (NF); 50) Nener et al. 1995 (BC); 51) Dubois et al. 1996 (QC)

Crepidostomum illinoiense Faust, 1918

Synonym: Crepidostomum hiodontos

Hunter and Bangham, 1932

Site: intestine

Hosts: Hiodon alosoides (5)

Hiodon tergisus $(1,3,4)$

Perca flavescens (2)

Distribution: $\quad \mathrm{MB}, \mathrm{ON}$ 
Records: 1) Hunter \& Bangham 1932

(ON); 2) Hopkins 1934 (ON); 3)

Bangham \& Hunter 1939 (ON); 4) Glenn 1980 (MB); 5) Szalai et al. 1992 (MB)

Crepidostomum isostomum Hopkins, 1931

Synonym: Crepidostomum canadense Hopkins, 1931

Includes: Crepidostomum laureatum of Cooper (1915) (partim)

Sites: gall bladder, intestine, pyloric caeca

Hosts: Etheostoma exile (1)

Perca flavescens $(3,9)$

Percina caprodes $(5,6)$

$6,7,8)$

Percopsis omiscomaycus $(2,4,5$,

Distribution: $\quad \mathbf{A B}, \mathrm{ON}, \mathrm{QC}$

Records: 1) Cooper 1915 (ON); 2)

Bangham \& Hunter 1939 (ON); 3) Lyster

1940a (QC); 4) Bangham 1955 (ON); 5)

Dechtiar 1972a (ON); 6) Dechtiar \&

Christie 1988 (ON); 7) Dechtiar et al.

1988 (ON); 8) Dechtiar \& Lawrie 1988

(ON); 9) Zelmer (AB - Beta Lake,

Burnstick Lake, Carburn Pond, Coal

Lake, Elkwater Lake, Fincastle Lake,

Joker Lake, Pigeon Lake, Pine Lake,

Rattlesnake Lake, Sherburne Lake,

Spruce Coulee Reservoir, Sylvan Lake)

Crepidostomum metoecus (Braun, 1900)

Braun, 1900

Sites: intestine, pyloric caeca

Hosts: Coregonus clupeaformis (1)

Cottus cognatus (1)

Esox lucius (1)

Oncorhynchus clarki (2)
Salvelinus namaycush (1)

Thymallus arcticus $(1,3)$

Distribution: BC, YT

Records: $\quad$ 1) Arthur et al. 1976 (YT); 2)

Margolis \& Moravec 1982 (BC); 3) Caira 1989 (BC, YT)

Crepidostomum percopsisi Nelson,

Choudhury and Dick, 1997

Site: intestine

Host: Percopsis omiscomaycus

Distribution: MB

Record: Nelson et al. 1997

Crepidostomum sp.

Sites: gall bladder, intestine, pyloric caeca

Hosts: Acipenser fulvescens (5)

Catostomus commersoni (2)

Coregonus clupeaformis $(8,9,10)$

Cottus cognatus (12)

Hiodon alosoides (4)

Lota lota $(3,11)$

Margariscus margarita (1)

Oncorhynchus mykiss (8)

Perca flavescens (7)

Salvelinus fontinalis (2)

Stizostedion vitreum $(6,7)$

Thymallus arcticus (3)

Distribution: $\quad \mathbf{A B}, \mathrm{BC}, \mathrm{MB}, \mathrm{ON}, \mathrm{QC}$

Records: 1) Bangham \& Venard

1946 (ON); 2) Fantham \& Porter 1948

(QC); 3) Bangham \& Adams 1954 (BC);

4) Kennedy \& Sprules 1967 (MB); 5)

Anthony 1974 (ON); 6) Anthony 1976

(ON); 7) Anthony 1978b (ON); 8) Pybus

\& Samuel 1978 [AB - Gregg Lake, Jarvis Lake (C. clupeaformis), Hardisty 
Creek (O. mykiss)]; 9) Samuel 1981

(AB - Blue Lake, Gregg Lake, Jarvis Lake); 10) Samuel 1985 (AB - Blue

Lake, Gregg Lake, Jarvis Lake); 11) Anthony 1987 (ON); 12) Dechtiar et al. 1989 (ON)

Creptotrema funduli Mueller, 1934

Includes: ?Allocreadium commune of Cooper (1915a) (partim)

Site: intestine

Hosts: Catostomus commersoni (1)

Perca flavescens $(2,3)$

Stizostedion canadense (3)

Stizostedion vitreum (3)

Distribution: $\quad \mathrm{MB}, \mathrm{ON}$

Records: 1) Cooper 1915 (ON); 2)

Szalai \& Dick 1991a (MB); 3) Szalai et

al. 1992 (MB)

Culaeatrema inconstans Lasee, Font and Sutherland, 1988

Synonym: Bunodera inconstans (Lasee,

Font and Sutherland, 1988) Brooks, 1992

Site: intestine

Host: Culaea inconstans

Distribution: (ON)

Records: Brooks 1992; McLennan \&

Shires 1995

Megalonia ictaluri Surber, 1928

Synonym: Crepidostomum ictaluri

(Surber, 1928) Van Cleave and Mueller 1934

Site: intestine

Host: Noturus flavus
Distribution: $\quad \mathrm{ON}$

Records: Bangham \& Hunter 1939;

Dechtiar \& Christie 1988

Plagiocirrus sp.

Site: unspecified

Host: Catostomus catostomus

Distribution: $\quad \mathrm{BC}$

Record: Bangham \& Adams 1954

Allocreadiidae gen. sp.

Site: intestine

Hosts: Acipenser fulvescens (1)

Noturus flavus (4)

Salvelinus fontinalis $(2,3)$

Distribution: LB, NF, ON

Records: 1) Bangham \& Hunter 1939

(ON); 2) Frost 1940 (NF); 3) Hicks \& Threlfall 1973 (LB); 4) Dechtiar \&

Christie 1988 (ON)

ORDER LEPOCREADIIFORMES

SUPERFAMILY LEPOCREADIOIDEA

FAMILY DEROPRISTIDAE

Deropristis hispida (Abilgaard in Rudolphi, 1819) Odhner, 1902

Site: intestine

Host: Acipenser fulvescens

Distribution: QC

Record: $\quad$ Stafford 1904 
Skrjabinopsolus manteri (Cable, 1950)

Cable, 1955

Site: intestine

Host: Acipenser fulvescens

Distribution: ON

Records: Anthony 1974; Dechtiar \& Christie 1988; Dechtiar et al. 1988;

Dechtiar \& Lawrie 1988

\section{FAMILY HOMALOMETRIDAE}

Homalometron armatum (MacCallum, 1895) Manter, 1947

Synonyms: Distomum isoporum var. armatum MacCallum, 1895; Anallocreadium armatum (MacCallum, 1895); Anallocreadium pearsei Hunter \& Bangham, 1932

Site: intestine

Host: Acipenser fulvescens

Distribution: ON

Record: MacCallum 1895

ORDER OPISTHORCHIIFORMES

SUPERFAMILY OPISTHORCHOIDEA

FAMILY CRYPTOGONIMIDAE

Acetodextra amiuri (Stafford, 1900) Pearse, 1924

Synonym: Monodistomum amiuri
Stafford, 1900

Site: gonads

Host: Noturus flavus

Distribution: ON

Record: Dechtiar 1972b

*Caecincola sp.

Site: digestive tract, eye

Host: Perca flavescens

Distribution: MB

Record: Szalai \& Dick 1991a, Szalai et al. 1992

*Centrovarium lobotes (MacCallum, 1895) Stafford, 1904

Synonym: Distomum lobotes MacCailum, 1895

Hosts: Esox lucius (6)

Notropis atherinoides (7)

Notropis hudsonius $(1,2,5,7)$

Percopsis omiscomaycus $(3,4,7)$

Stizostedion vitreum (6)

Distribution: $\mathrm{MB}, \mathrm{ON}$

Records: 1) Stewart-Hay 1951 (MB); 2)

Dechtiar 1972b (ON); 3) Dechtiar \&

Christie 1988 (ON); 4) Dechtiar et

al.1988 (ON); 5) Dechtiar \& Lawrie 1988 (ON); 6) Dechtiar \& Nepszy 1988 (ON);

7) Szalai et al. 1992 (MB)

Centrovarium lobotes (MacCallum 1895)

Stafford, 1904

Synonym: Distomum lobotes

MacCallum, 1895

Sites: intestine, pyloric caeca

Hosts: Esox lucius $(2,3,5,9,12,14,17$, 
19)

Notropis hudsonius $(6,16)$

Perca flavescens $(1,10,13,16$, 18,19)

Percopsis omiscomaycus $(4,19)$

Stizostedion canadense $(4,7,10$,

19)

Stizostedion vitreum $(1,2,3,4,5$,

\section{$6,11,13,17,19)$}

Distribution: $\quad \mathrm{MB}, \mathrm{ON}, \mathrm{QC}$

Records: 1) MacCallum 1895 (ON); 2)

Stafford 1904 (QC); 3) Lyster 1939 (QC);

4) Bangham \& Hunter 1939 (ON); 5)

Miller 1941 (QC); 6) Stewart-Hay $1951 b$

(MB); 7) Bangham 1955 (ON); 8)

Dickson 1964 (MB); 9) Dechtiar 1972b

(ON); 10) Dechtiar 1972a (ON); 11)

Anthony 1976 (ON); 12) Watson 1977

(MB); 13) Anthony 1978b (ON); 14)

Watson \& Dick 1980 (MB); 15) Anthony

1983 (ON); 16) Dechtiar et al. 1988

(ON); 17) Dechtiar \& Nepszy 1988

(ON); 18) Szalai \& Dick 1991a (MB); 19)

Szalai et al. 1992 (MB)

*Crytogoniumus chili Osborn, 1903

Site: musculature

Host: Notropis hudsonius

Distribution: ON

Record: Cooper 1915a

Cryptogonimus chili Osborn, 1903

Sites: intestine, pyloric caeca, stomach

Hosts: Notropis hudsonius (1)

Perca flavescens (2)

Distribution: ON

Records: 1) Cooper 1915; 2) Bangham \& Hunter 1939
FAMILY HETEROPHYIDAE

*Apophallus brevis Ransom, 1920

Synonym: Apophallus itascensis Warren, 1953

Includes: Distomum sp. of Cooper (1915)

Sites: fins, gills, musculature, subcutaneous

Hosts: Etheostoma exile $(8,19)$

Gusterosteus aculeatus (12)

Notropis hudsonius (17)

Perca flavescens $(1,8,9,10,11$, $13,14,15,16,17,18,19,20,21,22,23$, $24,25)$

Prosopium cylindraceum (11)

Salmo trutta $(3,7)$

7)

Salvelinus fontinalis $(2,3,4,5,6$,

Distribution: $\quad \mathrm{EXP}, \mathrm{MB}, \mathrm{NB}, \mathrm{NF}, \mathrm{NS}$, ON, QC, SK

Records: 1) Cooper 1915 (ON); 2)

Miller 1941 (QC); 3) Miller 1942 (QC);

4) Cameron 1945 (QC); 5) Cameron 1947 (QC); 6) Crossman 1962 (ON); 7)

Sandeman \& Pippy 1967 (NF); 8)

Dechtiar 1972a (ON); 9) Dechtiar 1972b

(ON); 10) Sinclair 1972a (ON); 11)

Sinclair 1972b (MB, ON, SK); 12) Hanek

\& Molnar 1974 (QC); 13) Pike \& Burt 1981 (NB); 14) Pike \& Burt 1982 (NB);

15) Pike \& Burt 1983 (NB); 16) Pike et

al. 1983 (NB); 17) Poole \& Dick 1983

(MB); 18) Poole \& Dick 1985 (MB); 19)

Dechtiar \& Christie 1988 (ON); 20)

Dechtiar et al. 1988 (ON); 21) Dechtiar \&

Lawrie 1988 (ON); 22) Dechtiar \&

Nepszy 1988 (ON); 23) Dechtiar et al.

1989 (ON); 24) Taylor et al.1993 (EXP) 
Includes: Apophallus brevis of Miller (1941); Apophallus brevis of Cameron (1945); Apophallus sp. of Lyster (1940)

Sites: fins, subcutaneous

Host: Salvelinus fontinalis

Distribution: NB, NF, NS, ON, QC

Records: Lyster 1940b (QC); Miller

1941 (QC); Cameron 1945 (QC); Sinclair 1972a (ON, QC); Hare \& Frantsi 1974

(NB); Cone \& Ryan 1984 (NF); Dechtiar et al. $1989(\mathrm{ON})$; Marcogliese \& Cone $1991 b(N F)$

*Apophallus venustus (Ransom, 1920) Cameron, 1936

Site: musculature

Hosts: Catostomus commersoni $(1,2)$

Esox lucius $(1,2)$

Moxostoma macrolepidotum (2)

Perca flavescens $(1,2,3,4,5)$

Stizostedion vitreum $(1,2)$

Distribution: ON. QC

Records: 1) Cameron 1937 (QC); 2)

Cameron 1945 (QC); 3) Tedla \&

Fernando 1969a (ON); 4) Tedla 1969

(ON); 5) Tedla \& Fernando 1972 (ON)

*Apophallus sp.

Site: subcutaneous

Hosts: Perca flavescens (4)

Salvelinus fontinalis $(1,2,3,5)$

Salvelinus namaycush (3)

Distribution: ON, QC

Records: 1) Lyster 1940a (QC); 2)

MacLulich 1943b (QC); 3) Bangham \&

Venard 1946 (ON); 4) Anthony 1978b
?Heterophyes $\mathrm{sp}$.

Site: unspecified

Host: Hiodon alosoides

Distribution: MB

Record: Kennedy \& Sprules 1967

${ }^{*}$ Heterophyidae gen. sp.

Sites: giils, musculature, subcutaneous

Hosts: Cottus cognatus (2)

Salvelinus fontinalis (1)

Distribution: NF, YT

Records: 1) Frost 1940 (NF); 2) Arthur et al. 1976 (YT)

\section{FAMILY OPISTHORCHIIDAE}

*Amphimerus elongatus Gower, 1938

Sites: fins, musculature

Host: Catostomus commersoni

Distribution: QC

Record: Cameron 1944

*Amphimerus pseudofelineus (Ward, 1901) Barker, 1911

Synonym: Opisthorchis pseudofelineus Ward, 1901

Site: musculature

Host: Catostomus commersoni

Distribution: MB

Records: Evans 1963a; Evans 1963b 
*Metorchis conjunctus (Cobbold, 1860) Looss, 1899

Site: musculature

Hosts: Catostomus catostomus $(4,8)$ Catostomus commersoni $(1,2,3,5$, $6,7,8)$

Perca flavescens (5)

Salvelinus fontinalis (8)

Distribution: AR, MB, ON, QC, SK

Records: 1) Cameron 1940 (MB, ON, QC, SK); 2) Cameron 1944 (QC); 3) Cameron 1945 (QC); 4) Rawson 1960 (SK); 5) Evans 1963b (MB); 6) Cameron \& Choquette 1963 (AR); 7) Eaton 1975 (SK); 8) Watson 1981 (QC)

Opisthorchis sp.

Site: intestine

Host: Esox lucius

Distribution: ON

Record: $\quad$ Cushing \& Bacal 1932

*Parametorchis complexus (Stiles and Hassall, 1894) Skrjabin, 1913

Synonyms: Parametorchis manitobensis Allen and Wardle, 1934; Parametorchis novaeboracensis Hung, 1926

Host: ?Catostomus commersoni

Distribution: $\quad \mathrm{MB}$

Record: $\quad$ Allen \& Wardle 1934

SUBORDER PLAGIORCHIATA

SUPERFAMILY GORGODEROIDEA
FAMILY GORGODERIDAE

Phyllodistomum coregoni Dechtiar, 1966

Sites: ureters, urinary bladder

Hosts: Coregonus clupeaformis (1, 2, 3 , $4,5,6,7,8,9)$ Prosopium cylindraceum (5)

Distribution: $\quad$ AB, LB, ON

Record: 1) Dechtiar 1966 (ON); 2) Dechtiar 1972a (ON); 3) Leong \& Holmes 1974a (AB - Cold Lake); 4) Leong 1975 (AB - Cold Lake); 5) Chinniah \& Threlfall 1978 (LB); 6) Leong \& Holmes 1981 (AB - Cold Lake); 7) Dechtiar \& Christie 1988 (ON); 8) Dechtiar et al. 1988 (ON); 9) Dechtiar \& Lawrie 1988 (ON)

Phyllodistomum folium (Olfers, 1816) Braun, 1899

Synonym: Distomum folium Olfers, 1816 Site: urinary bladder Host: Esox lucius Distribution: QC Record: Stafford 1904

Phyllodistomum lysteri Miller, 1940

Site: ureters

Hosts: Carpiodes cyprinus (9)

Catostomus catostomus $(7,10)$

Catostomus commersoni $(1,2,3,4$, $5,6,7,8,9,10$ )

Moxostoma macrolepidotum (7)

Distribution: ON, QC

Records: 1) Miller 1940b (QC); 2)

Dechtiar 1972a (ON); 3) Dechtiar 1972b 
(ON); 4) Molnar et al. 1974 (ON); 5)

Chan 1980 (ON); 6) Dechtiar \& Christie 1988 (ON); 7) Dechtiar et al. 1988 (ON);

8) Dechtiar \& Lawrie 1988 (ON); 9)

Dechtiar \& Nepszy 1988 (ON); 10)

Dechtiar et al.1989(ON)

Phyllodistomum superbum Stafford, 1904

Site: ureters

Hosts: Catostomus commersoni (3)

Esox lucius $(2,3)$

Perca flavescens $(1,4,5,6)$

Salvelinus fontinalis (3)

Stizostedion canadense (4)

Stizostedion vitreum (4)

Distribution: ON, QC

Records: 1) Stafford 1904 (QC); 2)

Lyster 1939 (QC); 3) Fantham \& Porter 1948 (QC); 4) Dechtiar 1972a (ON); 5)

Dechtiar et al. 1988 (ON); 6) Dechtiar \&

Lawrie 1988 (ON)

Phyllodistomum umblae (Fabricius, 1780) Bakke, 1982

Synonyms: Phyllodistomum limnosa

Sandeman and Pippy, 1967;

?Phyllodistomum lachancei Choquette, 1947

Sites: ureters, urinary bladder

Hosts: Coregonus clupeaformis (4)

Oncorhynchus mykiss $(6,7)$

$7,8,9)$

Salvelinus fontinalis $(1,2,3,4,5$,

Salvelinus namaycush (5)

Distribution: $\quad$ BC, LB, NF, ON, QC

Records: 1) Choquette 1947 (QC); 2)

Choquette 1948a (QC); 3) Sandeman \&

Pippy 1967 (NF); 4) Hicks \& Threlfall
1973 (LB); 5) Chinniah \& Threlfall 1978

(LB); 6) Bakke \& Bailey 1987 (BC); 7)

Dechtiar et al. (ON); 8) Wright et al. 1989

(QC); 9) Albert \& Curtis 1991 (QC)

\section{Phyllodistomum sp.}

Sites: ureters, urinary bladder

Hosts: Catostomus catostomus (1)

Coregonus artedii $(1,9)$

Esox lucius (8)

Moxostoma macrolepidotum (2)

Oncorhynchus mykiss $(3,6,7)$

Perca flavescens (4)

Phoxinus eos (11)

Phoxinus neogaeus (11)

Prosopium cylindraceum $(9,10)$

Ptychocheilus oregonensis (7)

Richardsonius balteatus (7)

Salvelinus namaycush $(3,6)$

Distribution: $\quad \mathrm{BC}, \mathrm{ON}, \mathrm{QC}$

Records: 1) Dechtiar 1972a (ON); 2)

Dechtiar 1972b (ON); 3) Anonymous

1978 (BC); 4) Anthony 1978b (ON); 5)

Frechette et al. 1978 (QC); 6) Arai \&

Mudry 1983 (BC); 7) Anonymous 1984

(BC); 8) Dechtiar \& Christie 1988 (ON);

9) Dechtiar et al. 1988 (ON); 10) Dechtiar

\& Lawrie 1988 (ON); 11) Dechtiar et al.

1989 (ON)

Gorgoderidae gen. sp.

Site: kidney

Host: Salvelinus fontinalis

Distribution: NF

Record: Frost 1940

SUPERFAMILY MICROPHALLOIDEA 
FAMILY MICROPHALLIDAE

Microphallus opacus (Ward, 1894) Ward, 1901

Synonym: Distomum opacus Ward, 1894

Site: intestine

Host: Perca flavescens

Distribution: ON, QC

Records: Bangham \& Hunter 1939

(ON); Fantham \& Porter 1948 (QC)

*Microphallidae gen. sp.

Site: unspecified

Hosts: Lota lota

Perca flavescens

Distribution: $\mathrm{ON}$

Record: Bangham 1941

\section{SUPERFAMILY OPECOELOIDEA}

\section{FAMILY LISSORCHIIDAE}

Lissorchis attenuatus (Mueller and Van

Cleave, 1932) Krygier and Macy, 1969

Synonym: Trigonodistomum attenuatum

Mueller and Van Cleave, 1932)

Site: intestine

Hosts: Carpiodes cyprinus $(5,6,16)$

Catostomus catostomus $(3,10,12$,

Catostomus commersoni $(1,2,4,7$,

$8,9,11,13,14,15,16,17)$

Catostomus macrocheilus $(3,10$,

12)

Moxostoma anisurum (5)

Distribution: $\quad \mathbf{A B}, \mathrm{BC}, \mathrm{ON}$
Records: 1) Bangham 1941 (ON); 2)

Bangham \& Venard 1946 (ON); 3)

Bangham \& Adams 1954 (BC); 4)

Bangham 1955 (ON); 5) Dechtiar 1972a (ON); 6) Dechtiar 1972b (ON); 7) Molnar et al. 1974 (ON); 8) Leong 1975 (AB -

Cold Lake); 9) Chan 1980 (ON); 10) Anonymous 1981 (BC); 11) Leong \&

Holmes 1981 (AB - Cold Lake); 12) Anonymous 1984 (BC); 13) Dechtiar \& Christie 1988 (ON); 14) Dechtiar et al. 1988 (ON); 15) Dechtiar \& Lawrie 1988 (ON); 16) Dechtiar \& Nepszy 1988 (ON); 17) Dubois et al. 1996 (QC)

Lissorchis crassicrurum Haderlie, 1953

Site: intestine

Hosts: Catostomus commersoni Hiodon alosoides Moxostoma anisurum

Distribution: MB

Record: $\quad$ Szalai et al. 1992

Lissorchis gullaris Self and Campbell, 1956

Site: intestine

Hosts: Carpiodes cyprinus (2)

Catostomus catostomus (1)

Distribution: LB, MB

Records: 1) Threlfall \& Hanek 1970a

(LB); 2) Szalai \& Dick 1987a (MB)

Lissorchis kritskyi Barnhart and Powell, 1979

Site: [intestine]

Host: Perca flavescens

Distribution: MB

Record: $\quad$ Poole \& Dick 1985 
Lissorchis simeri (Mueller and Van Cleave, 1932) Krygier and Macy, 1969

Site: digestive tract

Hosts: Catostomus catostomus (2) Catostomus commersoni (1)

Distribution: ON

Records: 1) Bangham 1941; 2) Bangham 1955

Lissorchis translucens (Simer, 1929)

Krygier and Macy, 1969

Site: intestine

Host: Catostomus commersoni

Distribution: ON

Record: Chan 1980

Lissorchis sp.

Includes: Triganodistomum sp. of Bangham (1941, 1955)

Site: unspecified

Hosts: Catostomus catostomus (1) Notropis hudsonius (2)

Distribution: ON

Records: 1) Bangham 1941; 2) Bangham 1955

\section{FAMILY OPECOELIDAE}

Allopodocotyle boleosomi (Pearse, 1924)

Kuntz and Font, 1984

Synonyms: Allocreadium boleosomi

Pearse, 1924; Podocotyle boleosomi

(Pearse, 1924) Yamaguti, 1971

Site: digestive tract
Host: Percina caprodes

Distribution: ON

Record: Bangham \& Hunter 1939

Plagioporus cooperi (Hunter and Bangham, 1932) Price, 1934

Synonym: Lebouria cooperi Hunter and Bangham, 1932

Includes: Allocreadium commune of Cooper (1915a) (partim)

Site: intestine

Hosts: Couesius plumbeus (4)

Notropis atherinoides $(2,4)$

Notropis hudsonius $(1,2,3,4)$

Rhinichthys cataractae $(1,2)$

Distribution: ON

Records: 1) Hunter \& Bangham 1932; 2)

Bangham \& Hunter 1939; 3) Bangham

1955 ; 4) Dechtiar et al. 1988

Plagioporus serotimus Stafford, 1904

Site: gall bladder

Hosts: Catostomus commersoni (2)

Moxostoma macrolepidotum $(1,2)$

Distribution: QC

Records: 1) Stafford 1904; 2) Miller

1940b

Plagioporus serratus Miller, 1940

Site: gall bladder

Host: Hiodon tergisus

Distribution: QC

Record: Miller 1940b

Plagioporus shawi (McIntosh, 1939) 
Margolis, 1972

Site: intestine

Host: Prosopium williamsoni

Distribution: $\quad \mathrm{BC}$

Record: Nener et al. 1995

Plagioporus sinitsini Mueller, 1934

Includes: Allocreadium commune of Cooper (1915a) (partim)

Sites: gall bladder, intestine

Hosts: Catostomus catostomus (2) Catostomus commersoni $(3,4,5)$

Hybognathus hankinsoni (4)

Notropis atherinoides (1)

Notropis hudsonius (7)

Phoximus eos (4)

Pimephales promelas $(4,5,6)$

Distribution: ON

Records: 1) Cooper 1915a; 2) Bangham

1955; 3) Dechtiar 1972a; 4) Kakonge

1972; 5) Molnar et al. 1974; 6) Chan

1980; 7) Dechtiar \& Christie 1988

Plagioporus sp.

Site: intestine

Host: Etheostoma exile

Distribution: ON

Record: Bangham \& Hunter 1939

SUPERFAMILY PLAGIORCHOIDEA

FAMILY LECITHODENDRIIDAE

?Pleurogenes sp.
Site: intestine

Host: ?Salvelinus fontinalis

Distribution: QC

Record: Fantham \& Porter 1948

FAMILY MACRODEROIDIDAE

Alloglossidium corti (Lamont, 1921) Van Cleave and Mueller, 1934

Site: intestine

Host: Noturus flavus

Distribution: ON

Records: Bangham 1972; Dechtiar \& Christie 1988

Alloglossidium geminum (Mueller, 1930)

Van Cleave and Mueller, 1934

Synonyms: Plagiorchis geminus

Mueller, 1930; Glossidium geminum

(Mueller, 1930) Yamaguti, 1954

Site: intestine

Hosts: Etheostoma exile (1)

Noturus flavus (2)

Perca flavescens (1)

Distribution: ON

Records: 1) Molnar et al. 1974; 2)

Dechtiar \& Christie 1988

*Digenea gen. sp.

Includes: Cercaria sp. of Cooper (1915a); Distomum sp. auct.

Sites: eye, fins, mesenteries, musculature, stomach, subcutaneous, viscera

Hosts: Catostomus commersoni (8) 
Esox lucius $(3,8,11)$

Gasterosteus aculeatus (9)

Margariscus margarita (6)

Notropis hudsonius (8)

Oncorhynchus mykiss (10)

Perca flavescens $(1,7,8)$

Phoxinus eos (4)

Rhinichthys cataractae (6)

Salvelinus fontinalis $(2,5,10)$

Stizostedion vitreum (3)

Distribution: $\quad$ AB, BC, MB, ON, QC

Records: 1) Cooper 1915 (ON); 2)

Ricker 1932 (ON); 3) Parnell 1934 (QC);

4) Bangham 1941 (ON); 5) MacLulich

1943b (ON); 6) Stewart-Hay 1951b

(MB); 7) Stewart-Hay 1951f(MB); 8)

Stewart-Hay 1952b (NB); 9) Lester 1975

(BC); 10) Mudry \& Anderson 1977

[AB - Edith Lake, (O. mykiss), Patricia

Lake (O. mykiss, $S$. fontinalis)]; 11)

Stock 1988 (AB - Jarvis Lake)

Digenea gen. sp.

Includes: Trematoda sp.; Distomum sp. auct.

Sites: digestive tract, urinary bladder, swim bladder

Hosts: Acipenser fulvescens (10)

Carpiodes cyprinus (5)

Catostomus catostomus (9)

Catastomus commersoni $(7,9)$

Coregonus artedii (11)

Coregonus clupeaformis $(4,6)$

Cottus cognatus $(8,9)$

Culaea inconstans (2)

Esox lucius $(9,12,13)$

Hiodon alosoides (11)

Lota lota $(9,12)$

Notropis hudsonius (9)

Perca flavescens $(1,9)$
Percopsis omiscomaycus (9)

Salvelinus fontinalis $(2,3)$

Distribution: $\quad$ AB, BC, MB, ON, QC

Records: 1) Cooper 1915a (ON); 2)

Ricker 1932 (ON); 3) Richardson 1935

(QC); 4) Bangham \& Hunter 1939 (ON);

5) Stewart-Hay $1951 \mathrm{~b}(\mathrm{MB})$; 6) Welch 1952 (ON); 7) Stewart-Hay 1952a (MB);

8) Bangham \& Adams 1954 (BC); 9)

Lawler 1959c (MB); 10) Harkness \&

Dymond 1961 (ON); 11) Reed 1962

(SK); 12) McAllister \& Mudry 1983

[AB - near Stony Plain, Unnamed Lake (E. lucius), Cold Lake (Lota lota), Bangs Lake ( $C$. artedii)]; 13) Stock 1988 (AB - Jarvis Lake)

\section{CLASS CESTODA}

\section{SUBCLASS CESTOIDEA}

\section{SUPERORDER EUCESTODA}

ORDER CARYOPHYLLIDEA

FAMILY CAPINGENTIDAE

Spartoides wardi Hunter, 1929

Site: intestine

Host: Carpiodes cyprinus

Distribution: MB, ON

Records: Stewart-Hay 1951b; Dechtiar 1972b (ON); Dechtiar \& Nepszy 1988 (ON) 


\section{FAMILY CARYOPHYLLAEIDAE}

Biacetabulum biloculoides Mackiewicz and McCrae, 1965

Site: intestine

Host: Catostomus commersoni

Distribution: ON

Records: Molnar et al. 1974; Chan 1980

Biacetabulum infrequens Hunter, 1929

Site: intestine

Host: Moxostoma anisurum

Distribution: $\quad \mathrm{MB}, \mathrm{ON}$

Records: Dechtiar 1972a (ON); Szalai et al. $1992(\mathrm{MB})$

Biacetabulum sp.

Site: intestine

Hosts: Carpiodes cyprimus (2)

Catostomus commersoni $(2,3,4)$

Moxostoma anisurum (4)

Pimephales promelas (1)

Distribution: $\quad \mathrm{MB}, \mathrm{ON}$

Records: 1) Dechtiar 1972a (ON); 2)

Dechtiar \& Nepszy 1988 (ON); 3)

Dechtiar et al. 1989 (ON); 4) Szalai et al. 1992 (MB)

Glaridacris catostomi Cooper, 1920

Includes: Caryophyllaeus terebrans of Bangham and Adams (1954) (partim); Glaridacris laruei of Bangham and

Venard (1946)

Sites: intestine, stomach
Hosts: Catostomus catostomus $(1,3,4,5$, $6,7,8,9,10,12,13,15,16,17,19,20$, $24,25,26,29,30$ )

Catostomus commersoni $(2,3$, $10,11,14,16,19,20,21,23,25,27,28$, $29,31,32$ )

26)

Catostomus macrocheilus $(10,24$,

Esox lucius $(18,22)$

Moxostoma macrolepidotum (28)

Distribution: $\quad \mathbf{A B}, \mathrm{BC}, \mathrm{MB}, \mathrm{NB}, \mathrm{NT}$, ON, QC, SK, YT

Records: 1) Wardle 1932 (ON, SK); 2)

Bangham \& Hunter 1939 (ON); 3)

Bangham 1941 (ON); 4) Miller 1944b

(NT); 5) Miller 1945e (AB - Lake

Athabasca); 6) Bangham \& Venard 1946

(ON); 7) Rawson \& Elsey 1950 (AB -

Pyramid Lake); 8) Rawson 1951 (NT);

9) Stewart-Hay 1953a (MB); 10)

Bangham \& Adams 1954 (BC); 11)

Bangham 1955 (ON); 12) Rawson 1959

(SK); 13) Mackiewicz 1965 (NT, SK);

14) Dechtiar 1972a (ON); 15) Hanek \&

Molnar 1974 (QC); 16) Mackiewicz

1976 (AB - Barrier Reservoir, Nose

Creek); 17) Arthur et al. 1976 (YT); 18)

Watson 1977 (MB); 19) Anonymous 1978 (BC); 20) Gordon et al. 1978 (QC); 21) Grey \& Mackiewicz 1980 (NB); 22)

Watson \& Dick 1980 (MB); 23) Chan 1980 (ON); 24) Anonymous 1981 (BC);

25) Arai \& Mudry 1983 (BC); 26)

Anonymous 1984 (BC); 27) Courtney

1987 (ON); 28) Dechtiar \& Christie 1988

(ON); 29) Dechtiar et al. 1988 (ON); 30)

Dechtiar \& Lawrie 1988 (ON); 31)

Dechtiar \& Nepszy 1988 (ON); 32) Li \&

Arai 1991 (AB - Chain Lakes)

Glaridacris confusus Hunter, 1929 
Site: intestine

Hosts: Carpiodes cyprinus (3) Catostomus commersoni $(1,2)$

Distribution: $\mathrm{ON}, \mathrm{QC}$

Records: 1) Bangham 1941 (ON); 2)

Richardson 1942 (QC); 3) Dechtiar 1972a

(ON)

Glaridacris laruei (Lamont, 1921) Hunter, 1927

Synonym: Glaridacris intermedius

Lamont, 1921

Site: intestine

Host: Catostomus commersoni

Distribution: ON, QC

Records: Lyster 1940a (QC); Bangham \& Venard 1946 (ON); Dechtiar 1972a (ON); Molnar et al. 1974 (ON); Gordon et al. 1978 (QC); Chan 1980 (ON);

Dubois et al. 1996 (QC)

Glaridacris terebrans (Linton, 1893)

Mackiewicz, 1974

Synonym: Caryophyllaeus terebrans (Linton, 1893) Hunter, 1930

Site: intestine

Hosts: Catostomus catostomus (2) Catostomus macrocheilus (2) Salvelinus confluentus (1) Salvelinus namaycush (1)

Distribution: $\quad \mathbf{A B}, \mathbf{B C}$

Records: 1) Rawson 1939 (AB - Lake

Minnewanka); 2) Bangham \& Adams 1954 (BC)

Glaridacris vogei Mackiewicz, 1976
Site: intestine

Host: Catostomus macrocheilus

Distribution: $\quad$ BC

Records: Mackiewicz 1976; Anonymous 1978; Arai \& Mudry 1983

Glaridacris sp.

Site: intestine

Hosts: Catostomus catostomus $(2,3,4)$ Catostomus commersoni $(1,2,4)$ Catostomus macrocheilus $(3,5)$ Notropis hudsonius (6)

Distribution: $\quad$ AB, BC, MB, ON

Records: 1) Stewart-Hay 1952b (MB);

2) Leong 1975 (AB - Cold Lake); 3) Anonymous 1978 (BC); 4) Leong \& Holmes 1981 (AB - Cold Lake); 5) Arai \& Mudry 1983 (BC); 6) Dechtiar \& Christie 1988 (ON)

Hunterella nodulosa Mackiewicz and McCrae, 1962

Site: intestine

Hosts: Catostomus catostomus $(13,14)$ Catostomus commersoni $(2,3,4,5$, $6,7,8,9,10,11,12,13,15,16,17)$ Catostomus macrocheilus $(1,8,11)$

Distribution: $\quad$ AB, BC, LB, MB, ON, QC Records: 1) Mackiewicz \& McCrae 1962 (BC); 2) Threlfall \& Hanek 1970a (LB); 3) Dechtiar 1972a (ON); 4) Mudry 1972 (AB - Bow River, Nose Creek); 5) Mudry \& Arai 1973a (AB - Bow River, Nose Creek); 6) Mudry \& Arai 1973b (AB - Bow River, Nose Creek); 7) Molnar et al. 1974 (ON); 8) Anonymous 1978 (BC); 9) Chan 1980 (ON); 10) Leong \& Holmes 1981 (AB - 
Cold Lake); 11) Arai \& Mudry 1983 (BC); 12) Dechtiar \& Christie 1988 (ON); 13) Li \& Arai 1988 [AB - Bow River, McGregor Lake, Nose Creek ( $C$. catostomus), Bow River, Chain Lake, Nose Creek (C. commersoni)]; 14) Dechtiar et al. 1989 (ON); 15) Li \& Arai 1991 (AB - Chain Lake); 16) Szalai et al. 1992 (MB); 17) Couillard et al. 1995 (QC)

Hunterella sp.

Site: intestine

Host: Catostomus commersoni

Distribution: $\quad \mathbf{A B}$

Record: Leong 1975 (AB - Cold Lake)

Hypocaryophyllaeus paratarius Hunter, 1927

Site: intestine

Host: Carpiodes cyprinus

Distribution: ON

Record: Bangham \& Hunter 1939

Isoglus idacris bulbocirrus Mackiewicz, 1965

Site: [intestine]

Host: Catostomus commersoni

Distribution: ON

Record: Courtney 1987

Isoglaridacris calentinei Mackiewicz, 1974

Includes: Caryophyllaeus terebrans of Bangham and Adams (1954) (partim)
Site: intestine

Host: Catostomus catostomus

Distribution: $\mathrm{BC}$

Record: Mackiewicz 1974

Monobothrium hunteri Mackiewicz, 1963

Includes: Glaridacris catostomi of Bangham and Adams (1954) (partim)

Site: intestine

Hosts: Carpiodes cyprinus $(3,4)$ Catostomus commersoni $(1,2)$

Distribution: $\quad \mathrm{BC}, \mathrm{MB}$

Records: 1) Mackiewicz 1963 (BC); 2) Molnar et al. 1974 (ON); 3) Szalai \& Dick 1987a (MB); 4) Szalai et al. 1992 (MB)

Monobothrium sp.

Site: intestine

Hosts: Catostomus catostomus $(1,2)$ Catostomus commersoni $(1,2)$

Distribution: $\quad \mathrm{BC}$

Records: 1) Anonymous 1978 ; 2) Arai \& Mudry 1983

Pliovitellaria wisconsinensis Fischthal, 1951

Site: intestine

Hosts: Phoxinus neogaeus Pimephales promelas

Distribution: ON

Record: Dechtiar et al. 1989

Rowardleus pennensis Mackiewicz and Deutsch, 1976 
Site: intestine

Host: Carpiodes cyprinus

Distribution: MB

Records: Szalai \& Dick 1987a; Szalai et al. 1992

\section{FAMILY LYTOCESTIDAE}

Khowia iowensis Calentine and Ulmer, 1961

Site: intestine

Host: Moxostoma anisurum

Distribution: $\quad \mathrm{MB}$

Record: $\quad$ Szalai et al. 1992

Caryophyllidea gen. sp.

Includes: Caryophyllaeidae gen. sp.

Site: intestine

Hosts: Catostomus catostomus $(1,2,3,4)$

Catostomus commersoni (3)

Catostomus macrocheilus $(2,4)$

Distribution: $\quad \mathbf{A B}, \mathrm{BC}, \mathrm{ON}$

Records: 1) Bangham 1955 (ON); 2)

Anonymous 1978 (BC); 3) Drouin 1982

(AB - McGregor Lake, Travers

Reservoir); 4) Arai \& Mudry 1983 (BC)

\section{ORDER CYCLOPHYLLIDEA}

\section{FAMILY DILEPIDIDAE}

*Paradilepis simoni Rausch, 1949

Site: mesenteries

Hosts: Oncorhynchus mykiss $(1,2,3)$
Prosopium williamsoni $(1,2,3)$

Ptychocheilus oregonensis $(1,2,3)$

Distribution: $\quad \mathrm{BC}$

Records: 1) Anonymous 1981 ; 2) Ching 1982; 3) Anonymous 1984

*Paradilepis sp.

Site: mesenteries

Host: Phoxinus neogaeus

Distribution: ON

Record: Dechtiar et al. 1989

FAMILY HYMENOLEPIDAE

*Hymenolepis sp.

Sites: coelom, liver

Hosts: Couesius plumbeus (1)

Etheostoma exile (2)

Distribution: $\quad \mathrm{BC}, \mathrm{ON}$

Records: $\quad$ 1) Bangham \& Adams 1954

(BC); 2) Bangham 1955 (ON)

\section{ORDER PROTOCEPHALIDEA}

\section{FAMILY PROTEOCEPHALIDAE}

Corallobothrium fimbriatum Essex, 1928

Site: intestine

Host: Noturus flavus

Distribution: ON

Records: Bangham \& Hunter 1939;

Decthiar 1972c; Dechtiar et al. 1988 
Corallobothrium sp.

Site: intestine

Hosts: Etheostoma exile

Perca flavescens

Distribution: ON

Record: Molnar et al. 1974

*Proteocephalus ambloplitis (Leidy, 1887) Benedict, 1900

Synonym: Proteocephalus micropteri

(Leidy, 1887) La Rue, 1911

Sites: mesenteries, viscera

Hosts: Culaea inconstans (9)

Notropis atherinoides (1)

Notropis hudsonius (1)

Notropis flavus (1)

Perca flavescens $(1,2,3,5,6,7$,

$8,9,10,11)$

Salvelinus fontinalis $(4,5)$

Salvelinus namaycush $(4,5)$

Stizostedion canadense (9)

Stizostedion vitreum $(1,3,9)$

Distribution: ON

Records: 1) Bangham \& Hunter 1939 , 2)

Bangham 1941;3) Detwiler 1941;4)

MacLulich 1943b; 5) Bangham \& Venard

1946; 6) Bangham 1955; 7) Tedla 1969;

8) Fischer \& Freeman 1973; 9) Dechtiar

1972a; 10) Tedla \& Fernando 1972; 11)

Dechtiar \& Nepszy 1988

Proteocephalus ambloplitis (Leidy, 1887) La

Rue, 1911

Synonym: Proteocephalus micropteri

(Leidy, 1887) La Rue, 1911

Site: viscera

Hosts: Culaea inconstans (3)
Margariscus margarita (3)

Perca flavescens $(1,2)$

Phoxinus eos (3)

Distribution: EXP, ON

Records: $\quad$ 1) Bangham 1941 (ON); 2)

Tedla \& Fernando 1972 (ON); 3) Fischer 1972 (EXP)

Proteocephalus coregoni Wardle, 1932

Site: intestine

Hosts: Coregonus artedii $(2,4,5,6)$

Coregonus clupeaformis $(3,4)$

Coregonus sp. (1)

Salvelinus namaycush (4)

Distribution: $\quad \mathrm{HBD}, \mathrm{MB}, \mathrm{ON}$

Records: 1) Wardle 1932 (HBD); 2)

Stewart-Hay 1951f (MB); 3) Stewart-Hay

1952b (MB); 4) Stewart-Hay 1953a

(MB); 5) Stewart-Hay $1953 b$ (MB); 6)

Anthony 1978a (ON)

Proteocephalus filicollis (Rudolphi, 1802)

Weinland, 1858

Site: intestine

Hosts: Coregomus artedii $(2,3,4,6)$

Coregonus clupeaformis (5)

Gasterosteus aculeatus (1)

Pungitius pungitius $(3,6)$

Distribution: $\quad$ AB, MB, NF

Records: 1) Hanek \& Threlfall 1970a

(NF); 2) Leong \& Holmes 1974a (AB -

Cold Lake); 3) Leong 1975 (AB - Cold

Lake); 4) Watson 1977 (MB); 5) Watson \& Dick 1979 (MB); 6) Leong \& Holmes 1981 (AB - Cold Lake)

Proteocephalus fluviatilis Bangham, 1925 
Site: intestine

Host: Perca flavescens

Distribution: ON

Record: Fischer 1972

Proteocephalus luciopercae Wardle, 1932

Site: intestine

Hosts: Stizostedion canadense $(1,9,16)$ Stizostedion vitreum $(1,2,3,4,5$, $6,7,8,9,10,11,12,13,14,15,16$ )

Distribution: $\quad \mathrm{MB}, \mathrm{ON}, \mathrm{SK}$

Records: 1) Wardle 1932 (MB, SK); 2)

Stewart-Hay 1951b (MB); 3) Stewart-

Hay 1951f (MB); 4) Stewart-Hay 1951g

(MB); 5) Stewart-Hay 1952b (MB); 6)

Stewart-Hay 1953a (MB); 7) Stewart-

Hay 1953b (MB); 8) Lawler 1954a

(MB); 9) Little 1954 (MB); 10) Rawson

1957a (SK); 11) Lawler \& Watson 1958

(MB); 12) Dickson 1964 (MB); 13)

Anthony 1976 (ON); 14) Anthony 1978b

(ON); 15) Poole \& Dick 1985 (MB); 16)

Szalai et al.1992 (MB)

Proteocephalus longicollis (Zeder, 1800)

Nufer, 1905

Synonyms: Proteocephalus pusillus

Ward, 1910; Proteocephalus exiguus La

Rue, 1911; Proteocephalus neglectus La

Rue, 1911; Proteocephalus laruei Faust,

1920; Proteocephalus arcticus Cooper,

1921; Proteocephalus wickliffi Hunter and

Bangham 1933; Proteocephalus

salmonidicola Alexander, 1951;

Proteocephalus tumidocollus Wagner, 1953

Sites: intestine, pyloric caeca

Hosts: Coregonus artedii $(4,5,6,8$,
$10,11,12,13,15,16,17,18,23,24,28$, $39,40,41,42,43$ )

Coregonus clupeaformis $(1,2,4$, $5,9,16,17,20,21,22,23,26,28,29$, $32,35,36,38,39,40,41$ )

Coregonus zenithicus (2)

Cottus cognatus (22)

Esox lucius $(22,38)$

Oncorhynchus clarki $(3,14)$

Oncorhynchus mykiss $(14,25$,

$30,33,35)$

Prosopium cylindraceum $(6,16$,

22, 37)

Prosopium williamsoni (14)

Salvelinus fontinalis $(18,19,26$,

27)

$33,35)$

34)

Salvelinus malma $(14,25,30$,

Salvelinus namaycush $(7,22,31$,

Thymallus arcticus (22)

Distribution: $\quad \mathbf{A B}, \mathrm{BC}, \mathrm{CA}, \mathrm{LB}, \mathrm{MB}, \mathrm{NT}$, ON, QC, SK, YT

Records: 1) Hart 1931 (ON); 2) Wardle 1932 (MB, SK); 3) Wardle 1933a (BC);

4) Hunter and Bangham 1933 (ON); 5)

Bangham \& Hunter 1939 (ON); 6)

Bangham 1941 (ON); 7) MacLulich 1943b (ON); 8) Miller 1944b (NT); 9)

Bangham \& Venard 1946 (ON); 10)

Rawson 1951 (NT); 11) Stewart-Hay 1951f(MB); 12) Stewart-Hay 1953a (MB); 13) Stewart-Hay 1953b (MB); 14) Bangham \& Adams 1954 (BC); 15) Little 1954 (MB); 16) Bangham 1955 (ON); 17) Dechtiar 1972a (ON); 18) Hicks \& Threlfall 1973 (LB); 19) Hanek \& Molnar 1974 (QC); 20) Leong \& Holmes 1974a (AB - Cold Lake); 21) Leong 1975 (AB - Cold Lake); 22) Arthur et al. 1976 (YT); 23) Watson 1977 (MB); 24) Anthony 1978a (ON); 25) Anonymous 
1978 (BC); 26) Chinniah \& Threlfall 1978 (LB); 27) Thompson \& Threlfall 1978 (QC); 28) Watson \& Dick1979 (MB); 29)

Leong \& Holmes 1981 (AB - Cold Lake); 30) Anonymous 1981(BC); 31)

Stewart \& Bernier 1982 (NT); 32)

Drouin 1982 (AB - McGregor Lake); 33) Arai \& Mudry 1983 (BC); 34) Stewart \& Bernier 1983 (NT); 35) Anonymous 1984 (BC); 37) Curtis 1988 (CA); 38) Stock 1988 (AB - Jarvis Lake); 39) Dechtiar \& Christie 1988 (ON); 40) Dechtiar et al. 1988 (ON); 41) Dechtiar \& Lawrie 1988 (ON); 42) Dechtiar et al. 1989 (ON); 43) Szalai et al. $1992(\mathrm{MB})$

Proteocephalus parallacticus MacLulich, 1943

Site: intestine Hosts: Salmo trutta $(1,2)$ Salvelinus fontinalis $(1,2,3,4,6)$ Salvelinus fontinalis $\mathrm{X}$ Salvelinus namaycush (6)

Distribution: $\quad \mathbf{A B}, \mathrm{ON}, \mathrm{QC}$

Records: 1) MacLulich 1943a (ON); 2) MacLulich 1943b (ON); 3) Bangham \& Venard 1946 (ON); 4) Choquette 1948a (QC); 5) Freeman 1961 (ON); 6) Freeman 1964a (ON); 7) Mudry \& Anderson 1977 (AB - Upper Waterton Lake); 8) Dechtiar 1972a (ON); 9) Dechtiar \& Lawrie 1988 (ON)

Proteocephalus pearsei La Rue, 1919

Site: intestine

Hosts: Coregomus artedii (13) Cottus ricei (10)
Lota lota $(4,16)$

Margariscus margarita (9)

Notropis atherinoides (8)

Perca flavescens $(1,2,3,4,5,6,7$, $8,10,11,14,15,18,19,20,21,22)$

Percina caprodes $(1,17)$

Percopsis omiscomaycus (1)

Stizostedion vitreum $(12,14)$

Distribution: EXP, MB, ON

Records: 1) Bangham \& Hunter 1939

(ON); 2) Bangham 1941 (ON); 3)

Bangham \& Venard 1946 (ON); 4)

Bangham 1955 (ON); 5) Tedla \&

Fernando 1969a (ON); 6) Tedla 1969

(ON); 7) Tedla \& Fernando $1972(\mathrm{ON})$;

8) Dechtiar 1972a (ON); 9) Fischer 1972 (EXP); 10) Cannon 1973 (ON); 11)

Fischer $1974(\mathrm{ON}) ; 12)$ Anthony 1976

(ON); 13) Anthony 1978a (ON); 14)

Anthony 1978b (ON); 15) Poole \& Dick 1985 (MB); 16) Anthony 1987 (ON); 17)

Dechtiar et al. 1988 (ON); 18) Dechtiar \& Lawrie 1988 (ON); 19) Dechtiar \&

Nepszy 1988 (ON); 20) Dechtiar \& Christie 1988 (ON); 21) Szalai \& Dick 1991a (MB); 22) Szalai \& Dick 1991a (MB); 22) Szalai et al. 1992 (MB)

Proteocephalus pinguis La Rue, 1911

Sites: intestine, pyloric caeca

Hosts: Esox lucius $(1,2,3,4,5,6,7$, $8,9,10,11,12,13,14,15,16,17,18$, $19,20,21,22,23,24,25,26,27,28,29$, $30,31,32,33,34,35,36,37$ )

Notropis atherinoides (4)

Notropis hudsonius $(4,37)$

Perca flavescens $(5,24)$

Stizostedion vitreum $(22,24,28)$

Distribution: $\quad$ AB, BC, LB, MB, NT, ON, QC 
Records: 1) Wardle 1932 (MB); 2)

Wardle 1936 (MB); 3) Richardson 1937

(QC); 4) Bangham \& Hunter 1939 (ON);

5) Cameron 1945 (QC); 6) Miller 1945e

(AB - Lake Athabasca); 7) Rawson 1951 (NT); 8) Stewart-Hay $1951 b$ (MB);

9) Stewart-Hay 1951f (MB); 10) Worley

\& Bangham 1952 (QC); 11) Stewart-Hay 1952b (MB); 12) Stewart-Hay 1953a

(MB); 13) Lawler 1954a (MB); 14)

Bangham \& Adams 1954 (BC); 15) Little 1954 (MB); 16) Bangham 1955 (ON); 17)

Lawler \& Watson 1958 (MB); 18)

Crossman 1962 (ON); 19) Threlfall \&

Hanek 1970b (LB); 20) Dechtiar 1972a

(ON); 21) Leong 1975 (AB - Cold

Lake); 22) Anthony 1976 (ON); 23)

Watson 1977 (MB); 24) Anthony 1978b

(ON); 25) Chinniah \& Threlfall 1978

(LB); 26) Thompson \& Threlfall 1978

(QC); 27) Watson \& Dick 1980 (MB);

28) Leong \& Holmes 1981 (AB - Cold

Lake); 29) Anthony 1983 (ON); 30)

Shostak \& Dick 1986 (MB); 31) Shostak

\& Dick 1987a (MB); 32) Dechtiar \&

Christie 1988 (ON); 33) Dechtiar et al. 1988 (ON); 34) Dechtiar \& Lawrie 1988

(ON); 35) Dechtiar \& Nepszy 1988 (ON);

36) Shostak \& Dick 1989a (MB); 37)

Szalai et al. 1992 (MB)

Proteocephalus ptychocheilus Faust, 1920

Site: intestine

Host: Ptychocheilus oregonensis

Distribution: BC

Records: Bangham \& Adams 1954; Anonymous 1978; Anonymous 1981; Arai \& Mudry 1983; Anonymous 1984
Proteocephalus pugetensis Hoff and Hoff, 1929

Site: intestine

Hosts: Culaea inconstans (3)

Gasterosteus aculeatus $(1,2,3)$

Distribution: $\quad \mathrm{BC}, \mathrm{ON}$

Records: 1) Bangham \& Adams 1954 (BC); 2) Lester 1975 (BC); 3) Dechtiar \& Christie 1988 (ON)

Proteocephalus singularis La Rue, 1911

Site: intestine

Host: Coregonus clupeaformis

Distribution: $\quad$ AB, MB

Records: Lawler1954a (MB); Little 1954 (AB - Bow Lake, Jackfish Lake, Lower Waterton Lake; MB) Lawler \& Watson 1958 (MB)

*Proteocephalus stizostethi Hunter and Bangham, 1933

Site: digestive tract

Hosts: Culaea inconstans

Hiodon tergisus

Perca flavescens

Percina caprodes

Distribution: ON

Record: Bangham \& Hunter 1939

Proteocephalus stizostethi Hunter and Bangham, 1933

Site: intestine

Hosts: Esox lucius $(3,8)$

Stizostedion canadense $(1,2,5)$

Stizostedion vitreum $(1,2,3,4,5$,

$6,7,9,10)$ 
Distribution: $\quad \mathrm{ON}, \mathrm{QC}$

Records: 1) Hunter \& Bangham 1933 (ON); 2) Bangham \& Hunter 1939 (ON);

3) Worley \& Bangham 1952 (QC); 4)

Bangham 1955 (ON); 5) Dechtiar 1972a

(ON); 6) Anthony $1976(\mathrm{ON})$; 7) Anthony

1978b (ON); 8) Anthony 1983 (ON); 9)

Dechtiar et al. 1988 (ON); 10) Dechtiar \&

Nepszy 1988 (ON)

**Proteocephalus sp.

Sites: intestine, pyloric caeca

Hosts: Carpiodes cyprinus (26)

Catostomus catostomus (57)

Catostomus ccmmersoni (3)

Coregonus artedii $(11,21,29,31$,

43)

Coregonus clupeaformis $(1,7$,

$12,21,28,29,31,34,36,39,43,44,46$, $47,51)$

Couesius plumbeus $(17,56)$

Culaea inconstans (2)

Esox lucius $(5,6,14,15,16,31$,

$38,39,40,43,44,51)$

Gasterosteus aculeatus $(22,30)$

Hiodon tergisus (2)

Lota lota $(8,21,24,31,41,52)$

Notropis atherinoides (2)

Notropis hudsonius $(2,18)$

Noturus flavus (55)

Oncorhynchus kisutch $(29,31$,

43)

$51,58,60)$

Oncorhynchus mykiss $(18,25)$

Perca flavescens $(14,35,39,44$,

Phoxinus neogaeus (26)

Pimephales promelas (27)

Prosopium cylindraceum $(17,28)$

Prosopium williamsoni $(25,34$,
$32,45,59)$

Pungitius pungitius $(23,26,31$,

Richardsonius balteatus (17)

Salvelinus fontinalis $(4,10,18,21$, $28,29,36,40,42,53,54,57$ )

Salvelinus fontinalis X Salvelinus namaycush $(37,56)$

Salvelinus namaycush $(9,19,21$, $28,29,36,48,49,50$ )

Stizostedion vitreum $(7,13,15,20$, $33,35)$

Distribution: $\quad$ AB, BC, LB, MB, NB, NF, NT, ON, QC, SK

Records: 1) Hart 1930 (ON); 2)

Bangham \& Hunter 1939 (ON); 3)

Bangham 1941 (ON); 4) Richardson 1942 (QC); 5) McLeod 1943 (MB); 6) McLeod 1944 (MB); 7) Miller 1945d (AB - Lake Athabasca, NT); 8) Bangham \& Venard 1946 (ON); 9) Miller \& Kennedy 1948 (NT); 10) Munroe 1949 (LB); 11)

Rawson 1951 (NT); 12) Stewart-Hay 1951f (MB); 13) Worley \& Bangham 1952 (QC); 14) Stewart-Hay 1952a (MB); 15) McTavish 1952 (MB); 16) Stewart-Hay 1953c (MB); 17) Bangham \& Adams 1954 (BC); 18) Bangham 1955 (ON); 19) Rawson 1961 (SK); 20) Ryder 1963 (AB - Iosegun Lake); 21) Freeman 1964a (ON); 22) Threlfall \& Hanek 1970a (LB, NF); 23) Threlfall \& Hanek 1970b (LB); 24) Threlfall \& Hanek 1971 (LB); 25) Bell \& Hoskins 1971 (BC); 26) Dechtiar 1972a (ON); 27) Kakonge 1972 (ON); 28) Hicks \& Threlfall 1973 (LB); 29) Leong \& Holmes 1974a (AB - Cold Lake); 30) Lester 1975 (BC); 31) Leong 1975 (AB - Cold Lake); 32) Dickinson \& Threlfall 1976 (LB); 33) Anthony 1976 (ON); 34) Anonymous 1978 (BC); 35) Anthony 1978b (ON); 36) Chinniah \& Threlfall 1978 (LB); 37) Dechtiar \& Berst 
1978 (ON); 38) Gordon et al. 1978 (QC); 39) Pybus \& Samuel 1978 (AB - Blue Lake, Cache Lake, Gregg Lake, Jarvis Lake); 40) Thompson \& Threlfall 1978 (QC); 41) Anonymous 1981 (BC); 42) Black 1981a (QC); 43) Leong \& Holmes 1981 (AB - Cold Lake); 44) Samuel 1981 [AB - Blue Lake, Cache Lake, Gregg Lake, Jarvis Lake (C. clupeaformis), Cache Lake, Jarvis Lake (E. lucius), Cache Lake (P. flavescens)]; 45) Curtis 1982 (QC); 46) Drouin 1982 (AB - Lake McGregor); 47) Arai \& Mudry 1983 (BC); 48) McAllister \& Mudry 1983 (AB - Cornwall Lake); 49) Stewart \& Bernier 1983 (NT); 50) Stewart \& Bernier 1984 (NT); 51) Samuel 1985 [AB - Blue Lake (C. clupeaformis, $E$. lucius), Cache Lake (C. clupeaformis, $E$. lucius, $P$. flavescens), Gregg Lake (C. clupeaformis), Jarvis Lake ( $C$. clupeaformis, E. lucius); 52) Anthony 1987 (ON); 53) Frimeth 1987a (NB); 54) Frimeth 1987b (NB); 55) Dechtiar \& Christie 1988 (ON); 56) Dechtiar et al. 1988 (ON); 57) Dechtiar \& Lawrie 1988 (ON); 58) Zelmer 1994 (AB Burntstick Lake, Carburn Pond, Elkwater Lake, Fincastle Lake, Garner Lake, Joker Lake, Pigeon Lake, Pine Lake, Rattlesnake Lake, Sherburne Lake, Sylvan Lake); 59) Marcogliese 1995 (NS); 60) Zelmer \& Arai 1998 (AB - Garner Lake)

Proteocephalidae gen. sp.

Site: intestine

Hosts: Acipenser fulvescens (5) Lota lota (4)
Salvelinus fontinalis $(1,2,3)$

Salvelinus namaycush (1)

Distribution: $\quad$ AB, MB, QC

Records: 1) Lyster 1940a (QC); 2)

Choquette 1948a (QC); 3) Hoffman \& Dunbar 1961 (QC); 4) Fillion 1961 (AB Lac Ste. Anne); 5) Choudhury \& Dick 1993 (MB)

\section{ORDER PSEUDOPHYLLIDEA}

SUBORDER BOTHRIOCEPHALATA

SUPERFAMILY AMPHICOTYLOIDEA

\section{FAMILY AMPHICOTYLIDAE}

Eubothrium crassum (Bloch, 1779) Nybelin, 1922

Synonyms: Abothrium crassum (Bloch, 1779); Eubothrium oncorhynchi Wardle, 1932

Sites: intestine, pyloric caeca

Hosts: Coregonus artedii $(3,5,7)$

Coregonus clupeaformis $(1,3)$

Lota lota $(1,3,4,6,7,9)$

Oncorhynchus mykiss (7)

Salvelinus fontinalis $(10,11)$

Salvelinus namaycush $(1,2,8)$

Distribution: $\quad$ LB, NB, NT, ON

Records:1) Cooper 1919 (ON); 2)

Cooper 1921 (ON); 3) Bangham \& Hunter 1939 (ON); 4) Bangham 1941 (ON); 5) Miller 1944b (NT); 6) Bangham \& Venard 1946 (ON); 7) Bangham 1955 (ON); 8) Hicks \& Threlfall 1973 (LB); 9) 
Anthony 1987 (ON); 10) Frimeth 1987a (NB); 11) Frimeth 1987b (NB)

Eubothrium rugosum (Batsch, 1786)

Nybelin, 1922

Sites: intestine, pyloric caeca

Host: Lota lota

Distribution: $\quad$ AB, BC, MB, NT, ON

Records: Wardle 1932 (MB); KuitunenEkbaum 1933 (MB); Miller 1944b (NT); Miller 1945e (AB - Lake Athabasca; NT); Rawson 1951 (NT); Stewart-Hay 1952b (MB); Stewart-Hay 1953b (MB); Bangham \& Adams 1954 (BC); Fillion 1961 (AB - Cold Lake, Lac La Nonne, Lac Ste. Anne); Dechtiar 1972a (ON); Leong 1975 (AB - Cold Lake); Mudry \& Anderson 1977 (AB - Upper Waterton Lake); Anonymous 1978 (BC); Leong \& Holmes 1981 (AB Cold Lake); Anderson \& Kennedy 1983 (BC); Anonymous 1984 (BC); Dechtiar et al. 1988 (ON); Dechtiar \& Lawrie 1988 (ON)

Eubothrium salvelini (Schrank, 1790)

Ny'jelin, 1922

Sites: intestine, pyloric caeca

Hosts: Coregonus artedii $(29,35)$

Coregonus clupeaformis $(11,29$,

31)

Oncorhynchus clarki (20)

Oncorhynchus kisutch $(29,37,39$, 42, 45, 47)

Oncorhynchus mykiss $(29,37,39$, $42,45,47)$

Prosopium cylindraceum $(27,31$, $53,54)$
Prosopium williamsoni $(33,41)$

Ptychocheilus oregonensis $(3,20)$

Salmo trutta (24)

Salvelinus confluentus $(1,6)$

Salvelinus fontinalis $(1,3,4,5,7$, $9,11,13,14,17,24,25,27,28,34,36$, $38,45,46,48,49,50,51,53,54,55,56$, $57,58,59)$

Salvelinus fontinalis $\mathrm{X}$ Salvelinus namaycush $(35,55)$

Salvelinus malma $(3,20,33,37$, 41, 42, 47)

Salvelinus namaycush $(1,2,3,8$, $9,10,11,12,15,16,18,19,20,21,22$, $23,26,27,29,30,31,32,33,34,36,39$, $40,41,43,44,46,47,48,52,53)$

Thymallus arcticus (31)

Distribution: $\quad \mathbf{A B}, \mathrm{BC}, \mathrm{LB}, \mathrm{MB}$, NB, NF, NT, ON, QC, SK, YT

Records: 1) Wardle 1932 (AB - Spray Lakes, MB, NT); 2) Wardle 1933b (ON); 3) Kuitunen-Ekbaum 1933 (AB - Spray Lakes); 4) Richardson 1936 (QC); 5) Richardson 1937 (QC); 6) Rawson 1939 (AB - Bow Lake, Moraine Lake, Lake Minnewanka); 7) Lyster 1940a (QC); 8) Richardson 1942 (QC); 9) MacLulich 1943b (ON); 10) Miller 1945e (AB Lake Athabasca, NT); 11) Bangham \& Venard 1946 (ON); 12) Miller 1946b (NT); 13) Choquette 1948a (QC); 14) Fantham \& Porter 1948 (QC); 15) Miller \& Kennedy 1948 (NT); 16) Rawson 1951 (NT); 17) Worley \& Bangham 1952 (QC); 18) Stewart-Hay 1953b (MB); 19) Stewart-Hay 1953b (MB); 20) Bangham \& Adams 1954 (BC); 21) Bangham 1955 (ON); 22) Rawson 1959 (SK); 23)

Rawson 1961 (SK); 24) Sandeman \& Pippy 1967 (NF); 25) Threlfall \& Hanek 1970c (NF); 26) Dechtiar 1972a (ON); 27) Hicks \& Threlfall 1973 (LB); 28) 
Hanek \& Molnar 1974 (QC); 29) Leong \& Holmes 1974a (AB - Cold Lake); 30) Leong 1975 (AB - Cold Lake); 31) Arthur et al. 1976 (YT); 32) Mudry \& Anderson 1977 [AB - Amethyst Lake (O. mykiss), Upper Waterton Lake (S. namaycush); 33) Anonymous 1978 (BC); 34) Chinniah \& Threlfall 1978 (LB); 35) Dechtiar \& Berst 1978 (ON); 36) Gordon et al. 1978 (QC); 37) Anonymous 1981 (BC); 38) Black 1981 (QC); 39) Leong \& Holmes 1981 (AB - Cold Lake); 40) Stewart \& Bernier 1982 (NT); 41) Arai \& Mudry 1983 (BC); 42) Anderson \& Kennedy 1983 (BC); 43) Stewart \& Bernier1983 (NT); 44) McAllister \& Mudry 1983 (AB - Cornwall Lake); 45) Cone \& Ryan 1984 (NF); 46) Stewart \& Bernier 1984 (NT); 47) Anonymous 1984 (BC); 48) Frimeth 1987a (NB); 49) Frimeth 1987b (NB); 50) Albert \& Curtis 1988 (QC); 51) Curtis 1988 (QC); 52)

Dechtiar \& Christie 1988 (ON); 53) Dechtiar \& Lawrie 1988 (ON); 54) Baggs \& McT. Cowan 1989 (NF); 55) Dechtiar et al. 1989 (ON); 56) Wright et al. 1989 (QC); 57) Albert \& Curtis 1991 (QC); 58) Marcogliese \& Cone 1991b (NF); 59) Poulin et al. 1992 (QC)

Eubothrium tulipai Ching and Andersen, 1983

Site: intestine

Host: Ptychocheilus oregonensis

Distribution: $\mathrm{BC}$

Records: $\quad$ Ching \& Andersen 1983; Anonymous 1984

Eubothrium sp.
Sites: intestine, pyloric caeca

Hosts: Catostomus catostomus $(7,8)$

Coregonus artedii (5)

Coregonus clupeaformis $(5,10)$

Couesius plumbeus (8)

Esox lucius $(6,7,12,15)$

Lota lota (3)

Oncorhynchus kisutch (11)

Oncorhynchus mykiss (15)

Prosopium cylindraceum (10)

Prosopium williamsoni (8)

Ptychocheilus oregonensis (13)

Pungitius pungitius $(11,14)$

Richardsonius balteatus (8)

Salvelimus fontinalis $(1,4,9,10)$

Salvelinus namaycush $(2,10)$

Stizostedion vitreum (6)

Distribution: $\quad \mathbf{A B}, \mathrm{BC}, \mathrm{LB}, \mathrm{MB}, \mathrm{NF}, \mathrm{NT}$, QC

Records: $\quad$ 1) Frost 1940 (NF); 2) Lyster 1940a (QC); 3) McLeod 1943 (MB); 4)

Munroe 1949 (LB); 5) Rawson 1951 (NT); 6) Stewart-Hay 1951g (MB); 7) McTavish 1953 (MB); 8) Bangham \& Adams 1954 (BC); 9) Threlfall \& Hanek 1970c (NF); 10) Hicks \& Threlfall 1973

(LB); 11) Leong 1975 (AB - Cold Lake); 12) Pybus \& Samuel 1978 (AB Cold Lake); 13) Anonymous 1978 (BC); 14) Leong \& Holmes 1981 (AB - Cold Lake); 15) McAllister \& Mudry 1983 (AB - Hines Creek Dugout)

\section{FAMILY BOTHRIOCEPHALIDAE}

Bothriocephalus claviceps (Goeze, 1782) Rudolphi, 1810

Site: intestine Hosts: Percopsis omiscomaycus 
Stizostedion canadense

Distribution: ON

Record: Bangham \& Hunter 1939

Bothriocephalus cuspidatus Cooper, 1917

Synonyms: Bothriocephalus cuspidatus

luciopercae Wardle, 1932;

Bothriocephalus cuspidatus hiodontos

Wardle, 1932; Bothriocephalus

cuspidatus cuspidatus Cooper, 1917

Sites: intestine

Hosts: Esox lucius $(2,4,6,18,31,36$,

43)

Etheostoma exile (3)

Hiodon alosoides $(2,20,23,28$,

Hiodon tergisus $(2,3)$

Notropis atherinoides $(3,27)$

Perca flavescens $(2,3,24,25,26$,

$27,31,32,37,38,42,43,44$ )

Percina caprodes (3)

Percopsis omiscomaycus (3)

Salvelinus malma (18)

Salvelinus namaycush $(9,11,12$,

18)

Stizostedion canadense $(2,3,19$, $27,43)$

Stizostedion vitreum $(1,2,3,5,7$, $8,9,10,12,13,14,15,16,17,18,19$, $21,22,27,29,30,33,34,35,37,38,39$, $40,41,43$ )

Distribution: $\quad$ AB, HBD, MB, NT, ON, QC, SK

Records: 1) Cooper 1919 (ON); 2) Wardle 1932 (HBD); 3) Bangham \& Hunter 1939 (ON); 4) McLeod 1943 (MB); 5) Miller 1944b (NT); 6) McLeod 1944 (MB); 7) Miller 1945e (AB - Lake Athabasca); 8) Rawson 1951 (SK); 9) Stewart-Hay 1951a (MB); 10) Stewart-
Hay 1951b (MB); 11) Stewart-Hay 1951c (MB); 12) Stewart-Hay 1951f (MB); 13) Stewart-Hay $195 \lg$ (MB); 14) Worley \& Bangham 1952 (QC); 15) Stewart-Hay 1952b (MB); 16) Stewart-Hay 1953a (MB); 17) Stewart-Hay 1953b (MB); 18) Little 1954 (AB - Bow Lake, Bow River, Waterton Lakes); 19) Bangham 1955 (ON); 20) Reed 1962 (SK); 21) Ryder 1963 (AB - Lac Ste. Anne, Iosegun Lake); 22) Dickson 1964 (MB); 23) Kennedy \& Sprules 1967 (AB Lake Claire, Red Deer River, South Saskatchewan River; MB, ON, SK); 24) Tedla \& Fernando 1969a (ON); 25) Tedla 1969 (ON); 26) Tedla \& Fernando 1972 (ON); 27) Dechtiar 1972a (ON); 28) Donald \& Kooyman 1974 (AB Chenal des Quatre Fourches, Lake Claire, Mamawi Lake, Prairie River); 29) Leong 1975 (AB - Cold Lake); 30) Anthony 1976 (ON); 31) Mudry \& Anderson 1977 (AB - Lower Waterton Lake); 32) Anthony 1978b (ON); 33) Pybus \& Samuel 1978 (AB - Gregg Lake); 34) Leong \& Holmes 1981 (AB Cold Lake); 35) Samuel 1981 (AB Gregg Lake); 36) Anthony 1983 (ON); 37) Poole \& Dick 1985 (MB); 38) Dechtiar \& Christie 1988 (ON); 39) Dechtiar et al. 1988 (ON); 40) Dechtiar \& Lawrie 1988 (ON); 41) Dechtiar \& Nepszy 1988 (ON); 42) Szalai \& Dick 1991a (MB); 43) Szalai et al. 1992 (MB); 44) Zelmer 1994 (AB - Garner Lake, Pigeon Lake, Sherburne Lake, Sylvan Lake)

Bothriocephalus formosus Mueller and Van Cleave, 1932 
Site: intestine

Hosts: Etheostoma exile $(1,2)$

Percina caprodes (2)

Percopsis omiscomaycus $(1,2,3)$

Distribution: ON

Records: 1) Bangham 1955; 2) Dechtiar 1972a; 3) Dechtiar et al. 1988

Bothriocephalus opsariichthydis Yamaguti, 1934

Synonyms: Bothriocephalus acheilognathi Yamaguti, 1934; Bothriocephalus fluviatilis Yamaguti, 1952; Bothriocephalus gowkongensis Yeh, 1955

Site: intestine

Host: Ptychocheilus oregonensis

Distribution: $\quad \mathrm{BC}$

Records: Anonymous 1978; Arai \& Mudry 1983

Bothriocephalus sp.

Sites: intestine, pyloric caeca

Hosts: Coregonus clupeaformis $(3,4,5)$

Esox lucius (6)

Gasterosteus aculeatus $(11,13)$

Hiodon tergisus (3)

Lota lota $(7,8,15)$

Myoxocephalus thompsoni (14)

Perca flavescens $(1,4,6,8,10$,

$12,16,17)$

Salvelinus namaycush $(3,7)$

Stizostedion vitreum $(2,9,13)$

Distribution: $\quad$ AB, BC, MB, NF, NT, ON

Records: 1) Bangham 1941 (ON); 2)

McLeod 1943 (MB); 3) Miller 1945e

(AB - Lake Athabasca; NT); 4)

Bangham \& Venard 1946 (ON); 5)
Rawson 1951 (NT); 6) Stewart-Hay 1952a (MB); 7) Bangham \& Adams 1954 (BC); 8) Bangham 1955 (ON); 9) Fillion 1961 (AB - Lac Ste. Anne); 10) Dickson 1964 (MB); 11) Hanek \& Threlfall 1970a (NF); 12) Cannon 1973 (ON); 13) Pybus \& Samuel 1978 (AB - Gregg Lake); 14) Black \& Lankester 1981a (ON); 15) Anthony 1987 (ON); 16) Zelmer 1994 (AB - Garner Lake, Pigeon Lake, Sherburne Reservoir, Sylvan Lake); 17) Zelmer \& Arai 1998 (AB - Garner Lake)

Bothriocephalidae gen. sp.

Sites: intestine, mesenteries

Hosts: Coregonus artedii (1) Gasterosteus aculeatus (2)

Pungitius pungitius (2)

Distribution: ON, QC

Records: 1) Pritchard 1931 (ON); 2)

Hanek \& Molnar 1974 (QC)

\section{FAMILY TRIAENOPHORIDAE}

*Triaenophorus crassus Forel, 1868

Synonyms: Triaenophorus robustus Olsson, 1893; Triaenophorus sp. type robustus of Cooper (1919);

Triaenophorus tricuspidatus morpha megadontus Wardle, 1932;

Triaenophorus tricuspidatus of Newton (1932) (partim)

Site: musculature

Hosts: Coregonus artedii $(2,4,5,6,7,8$, $9,10,11,12,14,15,16,17,18,19,22$, $23,24,25,26,27,30,31,32,35,40$, 
$45,47,50,51,54,55,57,60,61,62,63$, $65,67,69,70,71,72,74,75,76,77,78$, $80,82,83,84,85,86,90,91,94,95,96$, $97,99,100,103,104,105,106,108,112$, $113,114,115,118,119,120,128,130$, $131,133,134,135,136,138,139,140$, $141,144,151,157,158,159)$

Coregonus clupeaformis $(2,4$, $5,7,9,10,11,12,13,14,15,16,17,18$, $19,20,21,23,25,28,29,30,31,32,33$, $34,35,36,37,38,39,40,41,42,43,44$, $45,46,47,48,49,50,52,56,57,58,59$, $61,62,63,64,65,66,67,68,70,71,72$, $73,74,75,77,79,80,82,83,85,86,87$, $88,89,93,94,95,96,97,98,99,100$, $101,102,103,104,105,106,107,108$, $109,110,111,112,113,114,115,116$, $118,119,120,121,122,125,126,127$, $128,130,131,132,133,134,135,136$, $137,138,139,142,143,144,145,146$, $147,148,149,150,151,152,154,155$, $156,157,158,159)$

Coregonus zenithicus $(2,4,5,32$, $35,40,53,54,55,60,65,74,78,80,82$, 83,85 )

Coregonus sp. $(1,3,27,28,56,63$, $81,98,99,101,102,119,122,131)$

Lampetra japonica (129)

Oncorhynchus mykiss $(152,153)$

Perca flavescens (3)

Prosopium cylindraceum $(45,62$, $63,66,94,137,157$ )

Prosopium williamsoni $(11,18,25$, 133)

Prosopium sp. $(10,76)$

Salvelinus fontinalis $(32,63,118$, 135)

Salvelinus namaycush $(12,17,18$, $24,26,31,32,41,45,62,63,66,67,75$, $92,93,94,99,112,113,117,118,123$, $124,135,136,137,142,158)$

Thymallus arcticus $(24,27,30,62$,
$63,66,67,75,94,137)$

Distribution: $\quad \mathbf{A B}, \mathrm{BC}, \mathrm{CA}, \mathrm{EXP}, \mathrm{LB}$, MB, NT, ON, QC, SK, YT

Records: 1) Cooper 1919 (ON); 2) Bajkov 1932 (MB); 3) Wardle 1932 (MB); 4) Newton 1932 (MB); 5)

Nicholson 1932a (MB); 6) Fallis 1934 (ON); 7) Ekbaum 1935 (CA); 8) Kuitunen-Ekbaum 1936 (ON); 9) Detwiler 1941 (MB); 10) Miller 1943 (AB - Lesser Slave Lake); 11) Miller 1944a (AB - Cold Lake, Lesser Slave Lake); 12) Miller 1944b (NT); 13) Miller 1944c (AB - Lake Athabasca); 14) Miller 1944d (AB - Lesser Slave Lake); 15) Clemens et al. 1945 (BC); 16) Miller 1945b (AB - Lesser Slave Lake); 17) Miller 1945c (AB - Lake Athabasca); 18) Miller 1945d (AB - Lesser Slave Lake); 19) Doan 1945a (MB); 20) Doan 1945b (MB); 21) Doan 1945c (AB unspecified, MB, SK); 22) Doan 1945d (MB); 23) Doan 1945e (MB); 24) Miller 1946a (NT); 25) Miller 1946c (AB Lesser Slave Lake); 26) Miller 1946d (NT); 27) Miller \& Watson 1946 (AB Baptiste Lake); 28) Doan 1946a (MB); 29) Doan 1946b (MB); 30) Miller 1947 (NT); 31) Rawson 1947a (NT); 32) Rawson 1947b (AB - Lake Athabasca); 33) Doan 1947a (MB); 34) Doan 1947b (MB); 35) Miller 1948 (AB - Lesser Slave Lake); 36) Kennedy 1948a (MB); 37) Kennedy 1948 b (MB); 38) McMurtie \& Carter 1948 (CA); 39) Doan 1948a (MB); 40) Doan 1948b (MB); 41) Miller \& Kennedy 1948 (NT); 42) Oakland 1948 (MB); 43) Doan 1949a (MB); 44) Doan 1949b (MB); 45) Doan 1949c (AB - Lesser Slave Lake); 46) Oakland 1949 (MB); 47) Reed \& Dymond 1949 (MB); 48) Wheaton \& 
Rawson 1949a (SK); 49) Wheaton \& Rawson 1949b (SK); 50) Arnason 1950 (MB); 51) Miller 1950 (AB - Square Lake); 52) Oakland 1950 (MB); 53) Keleher 1950a (MB); 54) Keleher 1950b (MB); 55) Keleher 1950c (MB); 56) Doan 1950 (MB); 57) Lawler 1950a (MB); 58) Lawler 1950b (MB); 59) Rawson \& Wheaton 1950 (SK); 60) Welch 1950a (ON); 61) Welch 1950b (ON); 62) Rawson 1951 (NT); 63) Stewart-Hay 1951a (MB); 64) Wheaton \& Hazen 1951 (SK); 65) Keleher \& Matheson 1951 (MB); 66) Kennedy 1951 (MB); 67) Lawler 1951a (MB); 68) Lawler 1951b (MB); 69) Lawler 1951c (MB); 70) Welch 1951 (ON); 71) Doan 1951 (MB); 72) Lawler 1952b (MB); 73) Lawler 1952c (MB); 74) Reed \& Dymond 1951 (AB - Lesser Slave Lake, MB, ON); 75) Miller 1952 (AB Baptiste Lake, Calling Lake, Cold Lake, Lac la Biche, Lesser Slave Lake, Pinehurst Lake, Seibert Lake, Square Lake, Utikuma Lake, Winnifred Lake, MB); 76) Miller 1952b (AB - Square Lake); 77) Reed \& Dymond 1952 (MB); 78) Keleher 1952a (MB); 79) Keleher 1952b (MB); 80) Miller \& Johnson 1952 (AB - Square Lake); 81) Wheaton 1952 (NT); 82) Welch 1952a (ON); 83) Welch 1952b (ON); 84) Stewart-Hay 1952a (MB); 85) McTavish 1952 (MB); 86) McBurney \& Lawler 1953 (MB); 87) Lawler 1953b (MB); 88) Lawler 1953c (MB); 89) Lawler 1953d (MB); 90) Miller 1953 (AB - Square Lake); 91) Libin 1953a (ON); 92) Libin 1953b (EXP); 93) Stewart-Hay 1953a (MB); 94) Doan 1953 (AB - Lesser Slave Lake, MB, SK); 95) Reed 1953 (AB - Lesser Slave
Lake); 96) Reed \& Dymond 1953 (MB); 97) Miller 1954 (AB - Lesser Slave Lake); 98) Lawler \& Scott 1954 (MB, NT, ON); 99) Lawler 1954a (MB); 100) Kask 1954 (MB); 101) Lawler 1955 (MB); 102) Bangham 1955 (ON); 103) Fuller 1955 (NT); 104) Kask 1955 (MB); 105) Kask 1956 (MB); 106) Rawson 1957b (SK); 107) Kask 1958 (MB); 108) Lawler \& Watson 1958 (MB); 109) Lawler 1958b (MB); 110) Lawler 1959b (MB); 111) Kask 1959 (MB); 112) Rawson 1959 (SK); 113) Crampton 1960 (MB); 114) Kask 1960 (MB); 115) Lawler 1960b (MB); 116) Rawson 1961 (SK); 117) Lawler 1961a (MB); 118) Kask 1961 (MB); 119) Kask 1962 (MB); 120) Watson 1963b (MB); 121) Sunde 1963b (MB); 122) Watson 1963b (MB); 123) Dickson 1964 (MB); 124) Budde 1965 (MB); 125) Lawler 1965a (MB); 126) Lawler 1965b (MB); 127) Sunde 1965 (MB); 128) Buchwald \& Nursall 1969 (NT); 129) Freese 1969 (CA); 130) Lawler 1970 (MB); 131) Freese 1970 (MB); 132) Paetz \& Nelson 1970 (AB Lesser Slave Lake); 133) Freese 1971 (CA); 134) Dechtiar 1972a (ON); 135) Leong 1975 (AB - Cold Lake); 136) Anonymous 1976 (AB - Baptiste Lake, Brintnell Lake, Georges Lake, Kehiwin Lake, Muskwa Lake, Sandy Lake, Square Lake ( $C$. artedii); Amisk Lake, Bocquene Lake, Cold Lake, Equisetum Lake, Ethel Lake, Fawcett Lake, Heart Lake, Hilda Lake, Iosegun Lake, Lac la Biche, Lac la Nonne, Lesser Slave Lake, Little Sandy Lake, Long Lake II, Long Lake III, Marie Lake, May Lake, Moose Lake, Nipisi Lake (Little Whitefish Lake), Orloff Lake, Pearson Lake, Pinehurst Lake, Pound Lake, 
Rock Island Lake, Skeleton Lake, South Wabasca Lake, Twin Lakes, Winnifred Lake, Wolf Lake (C. artedii, C. clupeaformis); Cornwall Lake, Leland Lakes ( $C$. artedii, $C$. clupeaformis, S. namaycush); Arch Lake, Lake Athabasca, Bitscho Lake, Bourque Lake, Burnt Lake, Burstall Lake, Calling Lake, Carson Lake (McLeod Lake), Charles Lake, Lake Chipewyan, Christina Lake, Colin Lake, Florence Lake, Frenchman Lake, Frog Lake, Gift Lake, Gipsy Lake, Glover Lake, Gods Lake, Goodfish Lake, Graham Lake, Gregg Lake, Gregoire Lake, Grist Lake, Haig Lake, Ipiatik Lake, Jackfish Lake, Jarvis Lake, Jumbo Lake, Kirby Lake, Leggo Lake, Lodge Lake, Long Lake I, Loutit Lake, Margaret Lake, McLelland, Mink Lake, Namur Lake, North Wabasca Lake, Peerless Lake, Pegasus Lake, Piche Lake, Potts Lake, Primrose Lake, Richardson Lake, Roy-Emma Lake, Ryan Lake, Sander Lake, Sawn Lake, Seibert Lake, Touchwood Lake, Twin Lake, Utikuma Lake, Utikumasis Lake, Vandersteene Lake, Whitefish Lake, Wylies Lake ( $C$. clupeaformis); Tulip Lake ( $C$. clupeaformis, S. namaycush); 137) Arthur et al. 1976 (YT); 138) Watson 1977 (MB); 139) Dick \& Watson 1977 (MB); 140) Anthony 1978a (ON); 141) Chinniah \& Threlfall 1978 (LB); 142) Pybus \& Samuel 1978 (AB - Blue Lake, Cache Lake, Gregg Lake, Jarvis Lake); 143) Watson \& Dick 1979 (MB); 144) Leong \& Holmes 1981 (AB - Cold Lake); 145) Samuel 1981 (AB - Lake Athabasca, Blue Lake, Cache Lake, Calling Lake, Cold Lake, Gregg Lake,
Haig Lake, Jarvis Lake, Lac la Biche, North Wabasca Lake); 146) Dick \& Rosen 1982 (MB); 147) Rosen \& Dick 1982a (MB); 148) Rosen \& Dick 1982b (MB); 149) Bodaly et al. 1983 (MB); 150) Rosen \& Dick 1983 (MB); 151) Rosen \& Dick 1984a (MB); 152) Rosen \& Dick 1984b (EXP, MB); 153) Rosen \& Dick 1984c (MB); 154) Samuel 1985 (AB - Lake Athabasca, Blue Lake, Cache Lake, Calling Lake, Cold Lake, Gregg Lake, Haig Lake, Jarvis Lake, Lac la Eiche, North Wabasca Lake); 155) Dechtiar \& Christie 1988 (ON); 156) Dechtiar et al. 1988 (ON); 157) Dechtiar \& Lawrie 1988 (ON); 158) Nelson \& Paetz 1992 (AB unspecified); 159) Boily \& Curtis 1992 (QC)

Triaenophorus crassus Forel, 1868

Synonyms: Triaenophorus robustus Olsson, 1893; Triaenophorus sp. type robustus of Cooper (1919);

Triaenophorus tricuspidatus morpha megadontus Wardle, 1932

Sites: intestine, pyloric caeca

Host: Esox lucius

Distribution: $\mathbf{A B}, \mathrm{BC}, \mathrm{CA}, \mathrm{CCA}, \mathrm{HBD}$, LB, MB, NT, ON, QC, SK, YT

Records: Cooper 1919 (ON); Newton 1932 (MB); Wardle 1932 (MB); Fallis 1934 (ON); Ekbaum 1935 (CA); KuitinenEkbaum 1936 (MB, ON); Ekbaum 1937 (MB, ON); Detwiler 1941 (MB); Miller 1943 (AB - Lesser Slave Lake); Miller 1943a (AB -Cold Lake, Lesser Slave Lake); Miller 1944b (NT); Miller 1944d (AB - Lesser Slave Lake); Clemens et al. 
1945 (BC); Doan 1945a (MB); Doan 1945b (MB); Doan 1945e (MB); Miller 1945c (AB - Lesser Slave Lake); Miller 1945d (AB - Athabasca Lake); Doan 1946a (MB); Doan 1946b (MB); Miller 1946c (AB - Lesser Slave Lake); Miller \& Watkins 1946 (AB - Baptiste Lake); Doan 1947a (MB); Doan 1947b (MB); Miller 1947 (NT); Rawson 1947a (NT); Doan 1948a (MB); Doan 1948b (MB); Miller 1948 (AB - Lesser Slave Lake); Doan 1949a (MB); Doan 1949b (MR);Doan 1949c (AB - Lesser Slave Lake, ME, SK); Reed \& Dymond 1949 (MB); Wheaton \& Rawson 1949a (SK); Doan 1950 (MB); Lawler 1950a (MB); Miller 1950 (AB - Square Lake); Oakland 1950 (MB); Rawson \& Wheaton 1950 (SK); Welch 1950b (ON); Bangham 1951 (ON); Kennedy 1951 (MB, NT); Lawler 1951a (MB); Lawler 1951c (MB); Libin 1951a (AB- unspecified); Libin 1951b (AB - Baptiste Lake); Libin 1951c (AB - Baptiste Lake); Rawson 1951 (NT); Reed \& Dymond 1951 (MB); Wheaton \& Hazen 1951 (SK); Doan 1951 (MB); Lawler 1952b (MB); Lawler 1952c (MB); Libin 1952 (ON); McTavish 1952 (MB); Miller 1952b (AB - Baptiste Lake, Lesser Slave Lake, Square Lake); Reed \& Dymond 1952 (MB, ON); Stewart-Hay 1952b (MB); Welch 1952a (ON); Welch 1952b (ON); Miller \& Huston 1952 (AB - Baptiste Lake, Square Lake); Lawler 1953a (MB); Lawler 1953c (MB); Lawler \& McBurney 1953 (MB); Doan 1953 (CCA); Lawler 1953 (MB); McBurney \& Lawler 1953 (MB); Miller 1953 (AB - Square Lake); Reed \& Dymond 1953 (AB - Square Lake); Stewart-Hay 1953a (MB); Kask 1954 (MB); Lawler 1954a (MB); Lawler 1954c (MB); Lawler 1954e (MB); Lawler \& Scott 1954 (ON); Bangham 1955 (ON); Kask 1955 (MB); Kennedy 1955 (MB); Lawler 1955 (MB); Kask 1956 (MB); Lawler 1956a (MB); Lawler 1956c (MB); Watson \& Lawler 1956 (MB); Kask 1957 (MB); Lawler 1957 (MB); Rawson 1957b (SK); Kask 1958 (MB); Kennedy 1958 (MB); Lawler 1958a (MB); Lawler \& Watson 1958 (MB); Kask 1959 (MB); Lawler 1956d (MB); Kask 1960 (MB); Lawler 1960a (MB); Watson \& Price 1960 (MB, NT, ON); Kask 1961 (MB); Lawler 1961a (MB); Lawler 1961b (MB); Lawler 1961c (MB); Watson \& Lawler 1961 (MB); Kask 1962 (MB); Watson \& Lawler 1963 (MB); Watson \& Lawler 1965a (MB); Lawler 1965a (MB); Lawler 1965b (MB); Lawler 1965d (MB); Watson \& Lawler 1965a (MB); Watson \& Lawler 1965b (MB); Lawler 1966b (MB); Wilson \& Ronald 1967 (ON); Lawler 1970 (MB); Paetz \& Nelson 1970 (AB - unspecified); Dechtiar 1972a (ON); Leong 1975 (AB - Cold Lake); Anthony 1976 (ON); Arthur et al. 1976 (YT); Dick \& Watson 1977 (MB); Watson 1977 (MB); Anthony 1978b (ON); Chinniah \& Threlfall 1978 (LB); Pybus \& Samuel 1978 (AB - Blue Lake, Cacht Lake, Jarvis Lake); Thompson \& Threlfall 1978 (QC); Watson \& Dick 1980 (MB); Leong \& Holmes 1981 (ABCold Lake); Samuel 1981 (AB - Blue Lake, Cache Lake, Jarvis Lake); Dick \& Rosen 1982 (MB); Anthony 1983 (ON); Poole 1983 (MB); Rosen \& Dick 1984b (MB); Shostak et al. 1984 (MB); Samuel 1985 (AB - Blue Lake, Cache Lake, Jarvis Lake); Shostak \& Dick 1986 (MB); Shostak \& Dick 1987a (MB); Shostak \& Dick 1987b (MB); Shostak \& 
Dick 1987c (MB); Dechtiar et al. 1988 (ON); Dechtiar \& Lawrie 1988 (ON); Shostak \& Dick 1989a (MB); Shostak \& Dick 1989b (MB)

*Triaenophorus nodulosus (Pallas, 1760) Rudolphi, 1819

Includes: Triaenophorus sp. type nodulosus of Cooper (1919) (partim)

Sites: mesenteries, viscera

Hosts: Acipenser fulvescens (51) Carpiodes cyprimus $(53,54)$ Catostomus catostomus $(46,51)$ Catostomus commersoni $(1,3,40$, $42,50,51,53,54)$

Coregonus artedii $(41,42,57)$

Coregonus clupeaformis $(20,22$, $41,42,54,55)$

Cottus cognatus $(13,17,19,20$, $21,23,24,25,26,27,43,44)$

Lota lota $(4,5,6,7,8,10,11,13$, $14,15,16,18,21,23,24,25,26,27,28$, $31,32,38,43,44,46,56,57)$

Moxostoma anisurum $(38,39,40)$ Moxostoma macrolepidotum (51) Notropis hudsonius $(49,57)$ 48)

Oncorhynchus kisutch (41, 42, 43,

Perca flavescens $(1,2,10,11,12$, $13,14,15,16,17,18,21,23,24,25,26$, $27,29,30,31,32,33,34,35,36,37,38$, $40,41,45,46,49,50,51,53,54,55,56$, $57,58,59)$

Percopsis omiscomaycus (40)

Prosopium cylindraceum (51)

Pungitius pungitius (48)

Rhinichthys cataractae (52)

Salvelinus fontinalis X Salvelinus namaycush (45)

Salvelinus namaycush $(22,41,42$,
$43,48)$

Stizostedion canadense $(38,40)$

Thymallus arcticus (9)

Distribution: $\quad$ AB, MB, NT, ON, WCA, YT

Records: 1) Cooper 1919 (ON); 2) Ekbaum 1935 (WCA); 3) Bangham \& Hunter 1939 (ON); 4) Miller 1943 (AB Lesser Slave Lake); 5) Miller 1944a (AB - Lesser Slave Lake); 6) Miller 1945a (AB - Lesser Slave Lake); 7) Miller 1946c (AB - Lesser Slave Lake);

8) Doan 1945c (MB); 9) Miller 1946a (NT); 10) Lawler 1950a (MB); 11) Lawler 1950c (MB); 12) Stewart-Hay 1951c (MB); 13) Lawler 1951a (MB); 14) Reed \& Dymond 1951 (MB); 15) Welch 1952a (ON); 16) Welch 1952b (ON); 17) Lawler 1952a (MB); 18) Lawler 1952b (MB); 19) Reed \& Dymond 1952 (MB); 20) Stewart-Hay 1952a (MB); 21) Lawler 1953a (MB); 22) Stewart-Hay 1953a (MB); 23) Lawler 1953b (MB); 24) Lawler 1954a (MB);25) Lawler 1955 (MB);26) Lawler \& Watson 1958 (MB); 27) Lawler 1959a (MB); 28) Fillion 1961 (AB - Lac Ste Anne); 29) Lawler 1965c (MB); 30) Lawler 1966b (MB); 31) Lawler 1968 (MB); 32) Lawler 1969 (MB); 33) Tedla \& Fernando 1969a (ON); 34) Tedla \& Fernando 1969c (ON); 35) Tedla 1969 (ON); 36) Paetz \&

Nelson 1970 (AB - unspecified); 37)

Tedla \& Fernando 1972 (ON); 38)

Dechtiar 1972a (ON); 39) Dechtiar 1972b (ON); 40) Collins \& Dechtiar 1974 (ON); 41) Leong \& Holmes 1974a (AB - Cold Lake); 42) Leong 1975 (AB - Cold Lake); 43) Arthur et al. 1976 (YT); 44) Watson 1977 (MB); 45) Dechtiar \& Berst 1978 (ON); 46) Pybus \& Samuel 1978 (AB - Angling Lake); 47) Anthony 
1978b (ON); 48) Leong \& Holmes 1981 (AB - Cold Lake); 49) Poole \& Dick 1985 (MB); 50) Dechtiar \& Christie 1988 (ON); 51) Dechtiar et al. 1988 (ON); 52) Dechtiar \& Lawrie 1988 (ON); 53) Dechtiar \& Nepszy 1988 (ON); 54) Stock 1988 (AB - Jarvis Lake); 55) Szalai \& Dick 1991a (MB); 56) Nelson \& Paetz 1992 (AB - unspecified); 57) Szalai et al. 1992 (MB); 58) Zelmer 1994 (AB -

Garner Lake, Pigeon Lake, Sylvan Lake); 59) Zelmer \& Arai 1998 (AB - Garner Lake)

Triaenophorus nodulosus (Pallas, 1760) Rudolphi, 1819

Synonym: Triaenophorus tricuspidatus morpha microdentatus Wardle, 1932 Includes: Triaenophorus tricuspidatus of Newton (1932) (partim); Triaenophorus sp. type nodulosus of Cooper (1919) (partim)

Site: intestine

Hosts: Esox lucius (1, 2, 3, 4, 5, 6, 7, 8, 9, $10,11,12,13,14,15,16,17,18,19,20$, $21,22,23,24,25,26,27,28,29,30,31$, $32,33,34,35,36,37,38,39,40,41,42$, $43,44,45,46,47,48,49,50,51,52,53$, $54,55,56,57,58,60,61,62,63,66,67$, $68,69,70,71,72,73$ )

Stizostedion vitreum $(1,12,56,59$, $63,64,65$ )

Distribution: $\quad$ AB, CA, MB, NT, ON, SK, YT

Records: 1) Nicholson 1928 (MB); 2)

Newton 1932 (MB); 3) Wardle 1932 (MB); 4) Ekbaum 1935 (CA); 5) Miller 1943 (AB - Lesser Slave Lake); 6) Miller 1944a (AB - Lesser Slave Lake); 7) Miller 1944b (NT); 8) Miller 1944d
(AB - Lesser Slave Lake); 9) Miller 1945a (AB - Lesser Slave Lake); 10) Miller 1945c (AB - Lesser Slave Lake); 11) Miller 1945e (NT); 12) Lawler 1950a (MB); 13) Lawler 1950b (MB); 14) Rawson 1951 (NT); 15) Libin 1951b (AB - Baptiste Lake); 16) Libin 1951c (AB - Baptiste Lake); 17) Wheaton \& Hazen 1951 (SK); 18) Stewart-Hay 1952b (MB); 19) Miller 1952a (AB unspecified); 20) Welch 1952a (ON); 21) Welch 1952b (ON); 22) Lawler 1952a (MB); 23) Lawler 1952b (MB); 24) Stewart-Hay 1953a (MB); 25) Reed 1953 (MB); 26) Lawler \& McBurney 1953 (MB); 27) Lawler 1953a (MB); 28) Lawler 1953b (MB); 29) Lawler 1954a (MB); 30) Lawler 1955 (MB); 31) Lawler 1956 (MB); 32) Watson \& Lawler 1956 (MB); 33) Lawler \& Watson 1958 (MB); 34) Lawler 1958a (MB); 35) Lawler 1959b (MB); 36) Watson \& Price 1960 (MB); 37) Lawler 1960a (MB); 38) Lawler 1961b (MB); 39) Watson \& Lawler 1961 (MB); 40) Watson \& Lawler 1963 (MB); 41) Lawler 1963a (MB); 42) Watson 1963a (MB); 43) Lawler 1964a ( MB); 44) Watson \& Lawler 1965a (MB); 45) Watson \& Lawler 1965b (MB); 46) Lawler 1965b (MB); 47) Lawler 1965c (MB); 48) Lawler 196úb (MB); 49) Lawler 1968 (MB); 50) Lawler 1969 (MB); 51) Paetz \& Nelson 1970 (AB unspecified); 52) Dechtiar 1972a (ON); 53) Collins \& Dechtiar 1974 (ON); 54) Leong 1975 (AB - Cold Lake); 55) Arthur et al. 1976 (YT); 56) Anthony 1976 (ON); 57) Watson 1977 (MB); 58) Pybus \& Samuel 1978 (AB - Angling Lake); 59) Anthony 1978b (ON); 60) Watson \& Dick 1980 (MB); 61) Leong \& Holmes 1981 (AB - Cold Lake); 62) 
Anthony 1983 (ON); 63) Poole \& Dick 1985 (MB); 64) Samuel 1985 (AB Jarvis Lake); 65) Shostak \& Dick 1986 (MB); 66) Dechtiar \& Christie 1988 (ON); 67) Dechtiar \& Nepszy 1988 (ON); 68) Dechtiar et al. 1988 (ON); 69) Dechtiar \& Lawrie 1988 (ON); 70) Stock 1988 (AB - Jarvis Lake); 71) Shostak \& Dick 1989a (MB); 72) Nelson \& Paetz 1992 (AB - unspecified); 73) Szalai et al. $1992(\mathrm{MB})$

*Triaenophorus stizostedionis Miller, 1945

Includes: Triaenophorus sp. of Miller (1943)

Sites: coelom, gonads, mesenteries, musculature, viscera

Hosts: Cottus cognatus $(10,11,13,14$, 16)

Percopsis omiscomaycus (1, 2, 3, $4,5,6,7,8,9,10,11,12,14,15,16,17$, $18,19,20)$

Distribution: $\quad \mathbf{A B}, \mathbf{M B}, \mathrm{ON}$

Records: 1) Miller 1943a (AB - Lesser Slave Lake); 2) Miller 1945b (AB Lesser Slave Lake); 3) Lawler 1950a (MB); 4) Lawler 1950c (MB); 5) Lawler 1951a (MB); 6) Reed \& Dymond 1951 (MB); 7) Stewart-Hay 1951b (MB); 8) Welch 1952a (ON); 9) Welch 1952b (ON); 10) Lawler 1953b (MB); 11) Lawler 1954a (MB); 12) Lawler 1954b (MB); 13) Lawler \& Scott 1954 (ON); 14) Lawler \& Watson 1958 (MB); 15)

Paetz \& Nelson 1970 (AB unspecified); 16) Dechtiar 1972a (ON); 17) Dechtiar 1972b (ON); 18) Pybus \& Samuel 1978 (AB - Gregg Lake); 19) Dechtiar \& Christie 1988 (ON); 20) Nelson \& Paetz 1992 (AB - unspecified)

Triaenophorus stizostedionis Miller, 1945

Synonym: Triaenophorus sp. type nodulosus of Cooper (1919) (partim)

Includes: Triaenophorus sp. of Miller (1943)

Site: intestine

Host: Stizostedion vitreum

Distribution: $\quad$ AB, MB, NT, ON, SK

Records: Cooper 1919 (MB); Woodhead 1930 (MB); Miller 1943 (AB - Lesser Slave Lake); Miller 1945a (AB - Lesser Slave Lake); Miller 1945d (AB - Lake Athabasca); Lawler 1950a (MB); Lawler 1950气 (MB); Lawler 1951a (MB);

Rawson 1951 (NT); Stewart-Hay 1951b (MB); Lawler 1951b (MB); Welch 1952a (ON); Welch 1952b (ON); Reed \& Dymond 1953 (ON); Stewart-Hay 1953b (MB); Lawler 1954a (MB); Lawler 1954b (MB); Bangham 1955 (ON); Watson \& Lawler 1956 (MB); Rawson 1957a (SK); Lawler \& Watson 1958 (MB); Ryder 1963 (AB - Iosegun Lake); Dickson 1964 (MB); Watson \& Lawler 1965a (MB); Watson \& Lawler 1965b (MB); Paetz \& Nelson 1970 (AB unspecified); Dechtiar 1972a (ON); Anthony 1976 (ON); Anthony 1978a (ON); Pybus \& Samuel 1978 (AB Gregg Lake); Dechtiar \& Christie 1988 (ON); Dechtiar \& Nepszy 1988 (ON); Nelson \& Paetz 1992 (AB - unspecified)

*Triaenophorus sp.

Includes: Triaenophorus cuspidatus of Wardle (1932) (partim) 
Sites: musculature, viscera

Hosts: Catostomus catostomus $(17,24)$

Catostomus commersoni (1)

Coregonus artedii $(2,8,9,13,14$,

$16,17,22,23,26$ )

Coregonus clupeaformis $(2,8,9$,

$10,11,12,13,14,15,16,18,20,21,23$, $25,26,27,28,29,30,31,32,33)$

Coregomus zenithicus $(2,13)$

Esox lucius (3)

Perca flavescens $(1,3,4,19)$

Percopsis omiscomaycus $(4,6,7)$

Salvelinus namaycush $(5,12,13)$

Salvelinus canadense (4)

Stizostedion vitreum $(4,19)$

Distribution: $\quad$ AB, CA, MB, NT, ON, SK

Records: 1) Cooper 1919 (ON); 2)

Nicholson 1932a (MB); 3) Wardle 1932

(MB); 4) Bangham \& Hunter 1939 (ON);

5) McLeod 1943 (MB); 6) Miller 1943

(AB - Lesser Slave Lake); 7) Miller

1944a (AB - Lesser Slave Lake); 8)

Cameron 1945 (CA); 9) Rawson 1945

(MB); 10) Rawson 1946a (NT); 11)

Rawson 1946b (AB - Lake Athabasca);

12) Miller 1947 (NT); 13) Rawson 1947b

(AB - Lake Athabasca); 14) Wheaton \&

Rawson 1949a (SK); 15) Wheaton \&

Rawson 1949b (SK); 16) Miller \&

Macdonald 1950 (AB - Skeleton Lake);

17) Bangham 1951 (ON); 18) Stewart-

Hay 1951f(MB); 19) Stewart-Hay $1951 \mathrm{~g}$

(MB); 20) Welch 1951 (ON); 21)

Stewart-Hay 1952b (MB); 22) Stewart-

Hay 1953a (MB); 23) Stewart-Hay 1953b

(MB); 24) Bangham 1955 (ON); 25)

Rawson 1959 (SK); 26) Rawson 1960

(SK); 27) Sunde 1963a (MB); 28)

Sunde 1964 (MB); 29) Sunde 1965

(MB); 30) Koshinsky 1965 (SK); 31)

Freese 1973 (MB); 32) Eaton 1975

(SK); 33) McCart et al. (AB - Lake
Athabasca, Athabasca River)

Triaenophorus sp.

Includes: Triaenophorus sp. of Wardle (1933) (partim)

Site: intestine

Hosts: Esox lucius $(1,2,3,9,10,12,14$, $15,16,18,19)$

Stizostedion canadense (5)

Stizostedion vitreum $(4,5,6,7,8$,

$11,13,14,17)$

Distribution: $\quad \mathbf{A B}, \mathrm{MB}, \mathrm{NT}, \mathrm{ON}, \mathrm{SK}$

Records: 1) Cooper 1919 (ON); 2)

Nicholson 1932a (MB); 3) Wardle 1933b (MB); 4) Wardle 1933c (MB); 5)

Bangham \& Hunter 1939 (ON); 6) Miller 1943 (AB - Lesser Slave Lake); 7)

Miller 1944a (AB - Lesser Slave Lake);

8) Miller 1944b (NT); 9) Miller \&

Watkins 1945 (AB - Baptiste Lake); 10)

Wheaton \& Rawson 1949b (SK); 11)

Bangham 1951 (ON); 12) Libin 1951c

(AB - Baptiste Lake); 13) Stewart-Hay

1951f (MB); 14) Stewart-Hay 1951g

(MB); 15) Miller \& Huston 1952 (AB -

Baptiste Lake); 16) Stewart-Hay 1952a

(MB); 17) Fillion 1961 (AB - Lac Ste.

Anne); 18) McAllister \& Mudry 1983

(AB - Ross Lake); 19) Nelson \& Paetz

1992 (AB - unspecified)

SUBORDER DIPHYLLOBOTHRIATA

FAMILY DIPHYLLOBOTHRIIDAE

*Diphyliobothrium dendriticum (Nitzsch, 1824) Lühe, 1909 
Synonyms: Diphyllobothrium cordiceps (Leidy, 1824) Lühe, 1909

Sites: $\quad$ coelom, mesenteries

Hosts: Coregonus artedii $(1,4,10,13$, 14)

$10,14,15,16,19)$

Coregonus zenithicus (3)

Gasterosteus aculeatus (18)

Lota lota (12)

Oncorhynchus clarki (16)

Oncorhynchus mykiss $(2,7,11$,

16)

Prosopium villiamsoni $(7,16)$

Pungitius pungitius $(8,18)$

Salvelinus fontinalis $(11,17)$

Salvelinus malma $(2,7,11)$

Salvelinus namaycush $(3,6,9$,

$11,13,14)$

Distribution: $\quad \mathbf{A B}, \mathrm{BC}, \mathrm{CA}, \mathrm{MB}, \mathrm{NS}, \mathrm{NT}$, ON, QC

Records: 1) Thomas 1946 (ON); 2)

Anthony 1967 (BC); 3) Freeman \&

Thompson 1969 (ON); 4) Anthony 1978a

(ON); 5) Drouin 1982 (AB - McGregor

Lake, Travers Reservoir); 6) Stewart \&

Bernier 1983 (NT); 7) Anonymous 1984

(BC); 8) Curtis 1984 (NT); 9) Stewart \&

Bernier 1984 (NT); 10) Dick \& Poole

1985 (MB); 11) Anderson et al. 1987

(BC, QC); 12) Anthony 1987 (ON); 13)

Curtis 1988 (QC); 14) de Vos \& Dick 1989 (MB); 15) de Vos et al. 1990 (AB McGregor Lake); 16) Dais 1990 [AB McGregor Lake (C. clupeaformis), Rawson Lake ( $O$. clarki), Elbow Lake (S. fontinalis), BC]; 17) Albert \& Curtis 1991 (QC); 18) Marcogliese 1992 (NS);

19) Hobbs (undated) - (AB - Lesser

Slave Lake)
*Diphyllobothrium ditremum (Creplin, 1825) Lühe, 1910

Synonym: Diphyllobothrium osmeri (v. Linstow, 1878) Lühe, 1910

Sites: coelom, mesenteries

Hosts: Coregonus artedii $(2,10,11,12$, 14)

15)

Gasterosteus aculeatus $(8,9,13$,

Oncorhynchus mykiss $(1,9)$

Pungitius pungitius $(3,6,15,17)$

Salvelinus fontinalis $(9,13,16)$

Salvelinus malma (9)

Salvelinus namaycush $(4,5,7,9$,

13)

Distribution: $\quad$ BC, CA, NS, NT, ON, QC

Records: 1) Anthony 1967 (BC); 2)

Freeman \& Thompson 1969 (ON); 3)

Curtis 1982 (NT); 4) Stewart \& Bernier

1982 (NT); 5) Stewart \& Bernier 1983

(NT); 6) Curtis 1984 (NT); 7) Stewart \&

Bernier 1984 (NT); 8) Bérubé \& Curtis

1986 (QC); 9) Andersen et al. 1987 (BC);

10) Dechtiar \& Christie 1988 (ON); 11)

Dechtiar et al. $1988(\mathrm{ON})$; 12) Dechtiar \&

Lawrie 1988 (ON); 13) Curtis 1988 (CA);

14) Dechtiar et al. 1989 (ON); 15)

Marcogliese 1992 (NS); 16) Dubois et al. 1996 (QC); 17) Marcogliese 1995 (NS)

*Diphyllobothrium laruei Vergeer, 1942

Site: mesenteries

Hosts: Coregonus artedii

Coregonus zenithicus

Distribution: ON

Record: Vergeer 1942

*Diphyllobothrium latum (Linnaeus, 1758)

Cobbold, 1858 
Synonym: Dibothriocephalus latus (Linnaeus, 1758)

Includes: Diphyllobothrium sp. of

Rawson (1947)

Sites: coelom, intestine, musculature

Hosts: Coregonus clupeaformis (43)

Esox lucius (1, 2, 3, 4, 7, 8, 9,

$10,11,13,14,15,16,18,20,21,22,24$,

$25,26,27,28,29,30,32,33,34,37,38$,

$39,41,42,43$ )

Lota lota $(11,13,14,34)$

Oncorhynchus mykiss (37)

Perca flavescens $(9,11,13,14$,

$15,18,24,30,34,38,40)$

Salvelinus malma (37)

Salvelinus namaycush (26)

Stizostedion canadense $(11,13$,

$14,15,18)$

Stizostedion vitreum $(1,2,3,4,5$,

$6,7,8,11,12,13,14,15,16,17,18,19$, $20,21,22,23,25,26,27,29,30,31,32$, $33,35,36,37,38,39,40,41,43)$

Distribution: $\quad \mathbf{A B}, \mathrm{MB}, \mathrm{NT}, \mathrm{ON}, \mathrm{QC}, \mathrm{SK}$ Records: 1) Nicholson 1928 (MB); 2)

Nicholson 1929b (AB - unspecified), $\mathrm{MB}, \mathrm{ON}$ ); 3) Vergeer 1928a (AB unspecified), $\mathrm{MB}, \mathrm{ON})$; 4) Vergeer 1928b (AB - Lac la Biche, Lesser Slave

Lake, MB, NT, ON); 5) Vergeer 1928c (MB); 6) Magath 1928 (MB); 7) Vergeer 1929a (ON); 8) Vergeer 1929b (AB -

Lac la Biche, Lesser Slave Lake, MB, ON); 9) Magath \& Essex 1931 (MB); 10) Nicholson 1932 (MB); 11) Wardle 1932 (MB); 12) Cushing \& Bacal 1932 (MB);

13) Wardle 1933b (MB); 14) Bajkov

1933 (MB); 15) Wardle 1935 (MB); 16)

Miller 1944b (NT); 17) Miller 1945a

(AB - Lake Athabasca); 18) Miller

1945e (NT); 19 Rawson 1946a (MB); 20)

Rawson 1947a (AB - Lake Athabasca);

21) Rawson $1947 \mathrm{~b}$ (NT); 22) Rawson
1951 (NT); 23) Stewart-Hay $1951 \mathrm{f}(\mathrm{MB})$;

24) Stewart-Hay 1952a (MB); 25)

Stewart-Hay 1952b (MB); 26) Stewart-

Hay 1953a (MB); 27) Stewart-Hay 1953 b

(MB); 28) Stewart-Hay 1953c (MB); 29)

Lawler 1954a (MB); 30) Harvey 1955

(MB); 31 ) Rawson 1957a (SK); 32)

Lawler \& Watson 1958 (MB); 33)

Rawson 1960 (SK); 34) Roussow 1960

(QC); 35) Fillion 1961 (AB - Lac Ste.

Anne); 36) Ryder 1963 (AB - Iosegun

Lake); 37) Anthony 1967 (AB - Iosegun

Lake); 38) Paetz \& Nelson 1970 (AB -

Iosegun Lake); 39) Dick \& Poole 1985

(MB); 40) Poole \& Dick 1985 (MB); 41)

de Vos \& Dick 1989 (MB); 42) Dais

1990 (MB); 43) Nelson \& Paetz 1992

(Ab - unspecified)

\section{*Diphyllobothrium sp.}

Sites: coelom, heart, musculature, viscera

Hosts: Catostomus catostomus (16)

Coregonus artedii $(9,18,24,33$,

$38,40,44,47,49,50,53)$

Coregonus clupeaformis $(21,23$,

$30,33,34,38,40,43,44,45,46,47,48$,

$49,52,55,57,58,61,64,65,66,67,68)$

Coregonus zenithicus (35)

Esox lucius (10, 11, 12, 13, 19,21,

$22,25,44,50$ )

Gasterosteus aculeatus (32, 39,

55)

Lota lota $(1,2,4,42,51,58)$

Oncorhynchus clarki $(6,14,23$,

$51,53,57)$

Oncorhynchus kisutch $(23,38,40$,

Oncorhynchus mykiss $(20,23,24$,

$28,30,41,43,45,51,56,58,61,69)$ 
Perca flavescens $(16,25)$

Prosopium cylindraceum $(3,42)$

Prosopium williamsoni $(6,23,43$, $45,51,56,58)$

Pungitius pungitius (31)

Salmo trutta (28)

Salvelinus fontinalis $(2,3,16,17$, $27,28,36,37,43,46,59,62,63,68$ )

Salvelinus fontinalis X Salvelinus namaycush $(43,47)$

Salvelinus malma $(45,51,57)$

Salvelinus namaycush $(5,7,9,15$, $18,23,26,29,38,42,43,45,46,54,55$, $56,57,58,60,66$ )

Stizostedion vitreum $(8,10,11$, $12,13,19,21,22,24)$

Thymallus articus $(42,45,53,56)$

Distribution: $\quad \mathbf{A B}, \mathrm{BC}, \mathrm{LB}, \mathrm{MB}, \mathrm{NB}, \mathrm{NF}$, NS, NT, ON, QC, SK, YT

Records: 1) Cooper 1919 (NT); 2) Wardle 1932 (MB); 3) Wardle 1933c (NS); 4) Wardle 1935 (MB); 5) KuitunenEkbaum 1937a (ON); 6) Rawson 1939 (AB - Boom Lake, Bow Lake, Marvel Lake); 7) MacLulich 1943b (ON); 8) McLeod \& Moir 1944 (MB); 9) Miller 1945e (NT); 10) Rawson 1946a (MB);

11) Rawson 1946b (AB - Lake Athabasca); 12) Rawson 1947a (NT); 13) Rawson 1947b (AB - Lake Athabasca); 14) Rawson 1947c (AB Ma.vel Lake); 15) Miller \& Kennedy 1948 (NT); 16) Fantham \& Porter 1948 (QC); 17) Choquette 1948a (QC); 18) Rawson 1951 (NT); 19) Stewart-Hay 1951b (MB); 20) Rawson 1953 (AB Amethyst Lake); 21) Stewart-Hay 1953a (MB); 22) McTavish 1952 (MB); 23) Bangham \& Adams 1954 (BC); 24) Bangham 1955 (ON); 25) Hartman 1957 (AB - Iosegun Lake); 26) Freeman $1964 b(\mathrm{ON})$; 27) Lageaux
1966 (QC); 28) Sandeman \& Pippy 1967 (NF); 29) Freeman \& Thompson 1969 (ON); 30) Paetz \& Nelson 1970 (AB Lower Kananaskis Lake, Upper Kananaskis Lake); 31) Hanek \& Threlfall 1970b (NF); 32) Lester 1971b (BC); 33) Dechtiar 1972a (ON); 34) Dechtiar 1972b (ON); 35) Scott \& Crossman 1973 (ON); 36) Hicks \& Threlfall 1973 (LB); 37) Hanek \& Molnar 1974 (QC); 38) Leong \& Holmes 1974a (AB - Cold Lake); 39) Lester 1975 (BC); 40) Leong 1975 (AB - Cold Lake); 41) Hoskins et al. 1976 (BC); 42) Arthur et al. 1976 (YT); 43) Mudry \& Anderson 1977 [AB - Moraine Lake (O. mykiss, S. fontinalis X S. namaycush), Lower Waterton Lake, Pyramid Lake (P. williamsoni), Upper Waterton Lake (S. namaycush), Edith Lake, Horseshoe Lake, Moat Lake ( $O$. mykiss), Amethyst Lake, Herbert Lake (O. mykiss, $S$. fontinalis), Patricia Lake, Mud Lake, Shadow Lake, Vista Lake (S. fontinalis), Boom Lake ( $O$. clarki), BC, (O. clarki, S. fontinalis); 44)Watson 1977 (MB); 45) Anonymous 1978 (BC); 46) Chinniah \& Threlfall 1978 (LB); 47) Dechtiar \& Berst 1978 (ON); 48) Pybus \& Samuel 1978 (AB - Jarvis Lake); 49) Watson \& Dick 1979 (MB); 50) Watson \& Dick 1980 (MB); 51) Anonymous 1981 (BC); 52) Samuel 1981 (AB - Jarvis Lake); 53) Leong \& Holmes 1981 (AB - Cold Lake); 54) Stewart \& Bernier 1982 (NT); 55) Curtis 1982 (NT); 56) Arai \& Mudry 1983 (BC); 57) Stewart \& Bernier 1983 (NT); 58) Anonymous 1984 (BC); 59) Cone \& Ryan 1984 (NF); 60) Stewart \& Bernier 1984 (NT); 61) Samuel 1985 (AB - Jarvis Lake); 62) Frimeth 1987a (NB); 63) 
Frimeth 1987b (NB); 64) Dechtiar \& Christie 1988 (ON); 65) Dechtiar et al. 1988 (ON); 66) Dechtiar \& Lawrie 1988 (ON); 67) Stock 1988 (AB - Jarvis Lake); 68) Marcogliese \& Cone 1991b (NF); 69) Nelson \& Paetz 1992 (AB unspecified)

*Diphyllobothridae gen. sp.

Sites: coelom, heart, mesenteries, musculature, pericardium, viscera

Hosts: Loia lota (4)

Salvelinus fontinalis $(1,2,5)$

Stizostedion vitreum (3)

Distribution: MB, QC

Records: 1) Richardson 1936 (QC); 2)

Choquette 1948a (QC); 3) Stewart-Hay

1951 (MB); 4) Stewart-Hay 1953a

(MB); 5) Hoffman \& Dunbar 1961 (QC)

\section{FAMILY LIGULIDAE}

*Ligula intestinalis (Linnaeus, 1758)

Gmelin, 1790

Site: coelom

Hosts: Carpiodes cyprinus (37)

Catostomus catostomus $(2,7,11$,

$17,26,30,33,34,36,40$ )

Catostomus commersoni $(2,3,8,9$,

$10,11,12,13,15,16,17,18,19,21,23$,

$25,29,31,32,33,35,36,37,38,39,42)$

Catostomus macrocheilus (7)

Cottus cognatus $(24,27)$

Couesius plumbeus $(7,28)$

Culaea inconstans (2)

Gasterosteus aculeatus (1)

Hiodon alosoides $(37,39)$
Hybognathus hankinsoni (20)

Notropis hudsonius $(2,3,6,8,12$, $14,15,17,18,19,22,28,34,37,39,40$ )

Perca flavescens $(1,2,4,7,14,15$, $33,37,40,41$ )

Phoxinus eos (4)

Pimephales promelas $(20,36)$

Prosopium williamsoni (7)

Ptychocheilus oregonensis $(6,7$, $26,30)$

Richardsonius balteatus $(7,24,27)$

Salvelinus fontinalis $(5,7)$

Distribution: $\quad \mathbf{A B}, \mathrm{BC}, \mathrm{MB}, \mathrm{NB}, \mathrm{ON}$, QC, SK

Records: 1) Cooper 1919 (ON); 2)

Wardle 1932 (MB, SK); 3) Bangham \& Hunter 1939 (ON); 4) Bangham \& Venard 1946 (ON); 5) Choquette 1948a (QC); 6) Stewart-Hay 1951g (MB); 7) Bangham \& Adams 1954 (BC); 8) Lawler 1954a (MB); 9) Roussow 1954 (QC); 10) Bangham 1955 (ON); 11) Rawson 1957b (SK); 12) Lawler \& Watson 1958 (MB); 13) Rawson 1959 (SK); 14) Paetz \& Nelson 1970 (AB - unspecified); 15) Lawler 1964c (MB); 16) Dickson 1964 (MB); 17) Lawler 1965e (MB); 18) Lawler 1966a (MB); 19) Dechtiar 1972a (ON); 20) Kakonge 1972 (ON); 21) Molnar et al. 1974 (ON); 22) Mahon 1976 (তN); 23) Mudry \& Anderson 1977 (AB - Vista Lake); 24)

Anonymous 1978 (BC); 25) Chan 1980 (ON); 26) Anonymous 1981 (BC); 27) Arai \& Mudry 1983 (BC); 28) Drouin 1982 (AB - McGregor Lake (C. plumbeus), Travers Reservoir ( $N$. hudsonius); 29) Mackie et al. 1983 (ON); 30) Anonymous 1984 (BC); 31) Black \& Fraser 1984 (ON); 32) Dechtiar \& Christie 1988 (ON); 33) Dechtiar et al. 1988 (ON); 34) Dechtiar \& Lawrie 1988 
(ON); 35) Dechtiar \& Nepszy 1988 (ON); 36) Dechtiar et al. 1989 (ON); 37) Szalai et al. 1989 (MB); 38) Dais 1990 (AB McGregor Lake); 39) Szalai et al. 1992 (MB); 40 Nelson \& Paetz 1992 (AB unspecified); 41) Zelmer 1994 (AB Hastings Lake, Pigeon Lake); 42)

Dubois et al. 1996 (QC)

*Ligula sp.

Site: coelom

Host 3: Catostomus catostomus $(3,9,10$, $11,13)$

Catostomus commersoni $(2,9,10)$

Coregonus clupeaformis (5)

Culaea inconstans (5)

Esox lucius (4)

Lota lota (8)

Notropis hudsonius $(4,6,9)$

Rhinichthys cataractae $(5,10)$

Richardsonius balteatus (10)

Salvelinus fontinalis $(1,2)$

Distribution: $\quad$ AB, MB, ON, QC

Records: 1) Ricker 1932 (ON); 2)

Fantham \& Porter 1948 (QC); 3) Rawson \& Elsey 1950 (AB - Pyramid Lake); 4) Stewart-Hay 1952b (MB); 5) StewartHay 1953a (MB); 6) Lawler 1953b (MB); 7) Rawson 1959 (SK); 8) Fillion 1961 (AB - Lac Ste. Anne); 9) Lawler 1961c (MB); 10) Nelson 1964 (AB - Barrier Reservoir, Lower Kananaskis Lake, Upper Kananaskis Lake); 11) Paetz \& Nelson 1970 (AB - unspecified); 12) Samuel 1985 (AB - Cache Lake); 13)

Nelson \& Paetz 1992 (AB - unspecified)

FAMILY SCHISTOCEPHALIDAE
*Schistocephalus pungitii Dubinina, 1959

Site: coelom

Host: Pungitius pungitius

Distribution: NS

Records: Marcogliese 1992; Marcogliese 1995

*Schistocephalus solidus (Müller, 1776) Steenstrup, 1858

Site: coelom

Hosts: Cottus cognatus $(1,9,10,23)$

Cottus sp. (3)

Culaea inconstans $(5,8,9,10,16$, 33)

Esox lucius (24)

Gasterosteus aculeatus $(1,12,13$, $14,15,17,18,19,21,22,25,26,27,28$, $29,31)$

Notropis hudsonius (7)

Oncorhynchus kisutch (24)

Perca flavescens $(20,30)$

Pungitius pungitius $(2,19,24,32)$

Salvelinus fontinalis (11)

Salvelinus namaycush $(4,5)$

Distribution: $\quad \mathbf{A B}, \mathrm{BC}, \mathrm{LB}, \mathrm{MB}, \mathrm{NB}, \mathrm{NT}$, ON, QC, SK, YT

Records: 1) Cooper 1919 (ON); 2) Cooper 1921 (ON); 3) Wardle 1933c (QC); 4) Miller 1945e (NT); 5) Miller \& Macdonald 1950 (AB - Cottage Lake); 6) Rawson 1951 (NT); 7) Stewart-Hay 1951b (MB); 8) Stewart-Hay 1951d (MB); 9) Lawler 1954a (MB); 10) Lawler \& Watson 1958 (MB); 11) Sandeman \& Pippy 1967 (NF); 12) Morris \& Finnegan 1968 (BC); 13) Morris \& Finnegan 1969 (BC); 14) Threlfall 1968 (NF); 15) Hanek \& Threlfall 1970a (LB, NF); 16) Paetz \& Nelson 1970 (AB - unspecified); 17) 
Lester 1971b (BC); 18) Scott \&

Crossman 1973 (ON); 19) Hanek \&

Molnar 1974 (QC); 20) Molnar et al.

1974 (ON); 21) Lester 1975 (BC); 22)

Leong 1975 (AB - Cold Lake); 23)

Arthur et al. 1976 (YT); 24) Leong \&

Holmes 1981 (AB - Cold Lake); 25)

Curtis 1982 (NT); 26) Holloway \& Curtis 1982 (QC); 27) Reimchen 1982 (BC); 28)

McPhail \& Peacock 1983 (BC); 29) Cone \& Ryan 1984 (NF); 30) Poole \& Dick 1985 (MB); 31) Godin \& Sproul 1988

(NB); 32) Dechtiar et al. 1988 (ON); 33)

Dechtiar et al. 1989 (ON)

*Schistocephalus sp.

Site: coelom

Hosts: Catostomus catostomus (5)

Coregonus clupeaformis (1)

Culaea inconstans $(5,12)$

Gasterosteus aculeatus $(3,5,6)$

Notropis hudsonius (2)

Oncorhynchus mykiss (5)

Perca flavescens $(7,9)$

Prosopium williamsoni (5)

Pungitius pungitius $(4,8,10,11)$

Richardsonius balteatus (5)

Distribution: $\quad \mathbf{A B}, \mathrm{BC}, \mathrm{MB}, \mathrm{NB}, \mathrm{NT}$, ON, QC

Records: 1) Bangham \& Hunter 1939

(ON); 2) Miller \& Macdonald 1950

(AB- Conjuring Lake); 3) Dunbar \&

Hildebrand 1952 (QC); 4) Stewart-Hay

1952b (MB); 5) Bangham \& Adams 1954

(BC); 6) Manzer 1976 (BC); 7) Pybus \&

Samuel 1978 (AB - Cache Lake); 8)

Curtis 1981 (NT); 9) Samuel 1981 (AB -

Cache Lake); 10) Curtis 1982 (NT)
ORDER SPATHEBOTHRIDEA

FAMILY BOTHRIMONIDAE

Bothrimonas sturionis Duvernoy, 1842

Synonyms: Bothrimonas intermedius

Cooper, 1917; Diplocotyle olrikii Krabbe, 1874

Sites: intestine, pyloric caeca

Hosts: Coregonus artedii $(2,3)$

Coregonus clupeaformis $(2,3,6)$

Salmo trutta (5)

Salvelinus fontinalis $(2,3,4)$

Salvelinus malma (1)

Salvelinus namaycush (6)

Distribution: LB, MB, NB, NT

Records: 1) Cooper 1921 (NT); 2)

Wardle 1932 (MB); 3) Wardle 1933b

(MB); 4) Hicks \& Threlfall 1973 (LB); 5)

MacKinnon \& Burt 1982 (NB); 6)

Stewart \& Bernier 1983 (NT)

\section{FAMILY CYATHOCEPHALIDAE}

Cyathocephalus truncatus (Pallas, 1781)

Kessler, 1868

Synonym: Cyathocephalus americanus

Cooper, 1917

Sites: intestine, pyloric caeca

Hosts: Coregomus artedii $(12,13,14,16$, $18,19,20,24,30$ )

Coregonus clupeaformis $(1,2,3$, $4,5,6,7,8,9,10,11,13,14,16,17,18$, $19,20,24,26,27,29,30,31,33)$

Coregonus zenithicus (30)

Cottus ricei (30) 
Esox lucius $(18,21)$

Gasterosteus aculeatus $(15,25)$

Lota lota $(16,24)$

Myoxocephalus thompsoni (23)

Oncorhynchus kisutch $(14,16$,

24)

30)

Oncorhynchus mykiss $(22,27)$

Perca flavescens $(12,13,19,29$,

Prosopium cylindraceum $(17,30)$

Salvelinus confluentus (4)

Salvelinus fontinalis (32)

Salvelinus fontinalis X Salvelinus namaycush $(19,30)$

Salvelinus malma $(22,27)$

Salvelinus namaycush $(2,14,17$, $24,28)$

Distribution: $\quad \mathbf{A B}, \mathrm{BC}, \mathrm{CA}, \mathrm{MB}, \mathrm{NT}$, ON, YT

Records: 1) Cooper 1919 (ON); 2) Cooper 1921 (NT); 3) Hart 1931 (ON);

4) Wardle 1932 (AB - Spray Lakes, MB); 5) Bangham \& Hunter 1939 (ON);

6) Miller 1944b (NT); 7) Miller 1944e (NT); 8) Miller 1945e (AB - Lake

Athabasca); 9) Rawson 1951 (NT); 10) McTavish 1952 (MB); 11) Bangham 1955 (ON); 12) Dechtiar \& Loftus 1965 (ON); 13) Dechtiar 1972a (ON); 14) Leong \&

Holmes 1974a (AB - Cold Lake); 15) Lester 1975 (BC); 16) Leong 1975 (AB Cold Lake); 17) Arthur et al. 1976 (YT); 18) Watson 1977 (MB); 19) Dechtiar \& Berst 1978 (ON); 20) Watson \& Dick 1979 (MB); 21) Watson \& Dick 1980 (MB); 22) Anonymous 1981 (BC); 23) Black \& Lankester 1981a (ON); 24) Leong \& Holmes 1981 (AB - Cold Lake); 25) Reimchen 1982 (BC); 26) McCart et al. 1982 (AB - Athabasca River, Lake Athabasca); 27) Anonymous 1984 (BC); 28) Stewart \&
Bernier 1984 (NT); 29) Dechtiar \& Christie 1988 (ON); 30) Dechtiar et al. 1988 (ON); 31) Dechtiar \& Lawrie 1988 (ON); 32) Curtis 1988 (CA); 33) Hobbs (undated) (AB - Lesser Slave Lake)

Cyathocephalus sp.

Site: digestive tract

Hosts: Stizostedion vitreum (2) Thymallus arcticus (1)

Distribution: AB, NT

Records: 1) Miller 1946a (NT); 2)

Pybus \& Samuel 1978 (AB - Cold

Lake)

ORDER TETRAPHYLLIDEA

FAMILY PHYLLOBOTHRIIDAE

Phyllobothrium caudatum (Zschokke and Heitz, 1914) Zmeev, 1936

Synonyms: Phyllobothrium salmonis Fujita, 1922; Phyllobothrium ketae

Canavan, 1928

Sites: intestine, pyloric caeca

Hosts: Oncorhynchus kisutch $(1,2)$ Salvelinus malma (2)

Distribution: $\quad \mathrm{BC}$

Records: 1) Anonymous $1981 ; 2$ )

Anonymous 1984

Phyllobothrium sp.

Site: unspecified

Host: Salvelinus malma 
Distribution: $\quad \mathrm{BC}$

Record: Bangham \& Adams 1954

Tetraphyllidea gen. sp.

Site: intestine

Hosts: Acipenser fulvescens (3)

Salvelinus fontinalis $(1,2)$

Distribution: MB, NB

Records: 1) Frimeth 1987a (NB); 2)

Frimeth $1987 \mathrm{~b}(\mathrm{NB})$; 3) Choudhury \&

Dick 1993 (MB)

${ }^{*}$ Cestoidea gen. sp.

Sites: mesenteries, musculature, viscera

Hosts: Coregonus artedii (1)

Coregonus clupeaformis (2)

Perca flavescens $(3,4)$

Salvelinus namaycush $(5,6)$

Distribution: $\quad \mathbf{A B}, \mathrm{ON}, \mathrm{NT}, \mathrm{QC}$

Records: 1) Pritchard 1931 (ON); 2)

McLeod 1943 (MB); 3) Fantham \&

Porter 1948 (QC); 4) Harvey 1955 (MB);

5) Cuerrier \& Schultz 1957 (AB -

Lower Waterton Lake, Upper

Waterton Lake); 6) Stewart \& Bernier

1983 (NT)

Cestoidea gen. sp.

Site: digestive tract

Hosts: Acipenser fulvescens (13)

Catostomus catostomus $(17,20)$

Coregonus artedii (11)

Coregonus clupeaformis $(2,5,8$, $11,19,21)$

Esox lucius (5, 9, 10, 11, 12, 18,

22)
Gasterosteus aculeatus (14)

Moxostoma anisurum (5)

Oncorhynchus mykiss $(17,20)$

Perca flavescens (7)

Ptychocheilus oregonensis (20)

Salvelinus confluentus $(1,4)$

Salvelimus fontinalis $(3,15)$

Salvelinus malma $(17,20)$

Salvelinus namaycush (3)

Stizostedion canadense (11)

Stizostedion vitreum $(5,6,10,11$, $16,18)$

Distribution: $\quad \mathbf{A B}, \mathbf{B C}, \mathrm{MB}, \mathrm{NF}, \mathrm{NS}, \mathrm{NT}$, ON, QC, SK

Records: 1) Neave \& Bajkov 1929

[AB-Annette Lake ( $C$. catostomus),

Jacques Lake (S. confluentus); 2)

Bajkov 190 (MB); 3) Ricker 1932 (ON);

4) Rawson 1939 (AB - Mystic Lake); 5)

McLeod 1943 (MB); 6) McLeod \& Moir

1944 (MB); 7) Fantham \& Porter 1948

(QC); 8) Wheaton \& Hazen 1951 (SK);

9) Stewart-Hay 1951e (MB); 10)

Stewart-Hay 1951h (MB); 11) McTavish

1952 (MB); 12) Stewart-Hay 1953b

(MB); 13) Harkness \& Dymond 1961

(ON); 14) Threlfall 1968 (NF); 15)

Frantsi et al. 1975 (NS); 16) Pybus \&

Samuel 1978 (AB - Cold Lake); 17)

Anonymous 1981 (BC); 18) Kinnis \&

Curtis 1981 (QC); 19) McAllister \&

Mudry 1983 [AB - McGregor Lake (C. clupeaformis); Cornwall Lake (S. namaycush)]; 20) Anonymous 1984 (BC); 21) Stewart \& Bernier 1984 (NT); 22) Samuel 1985 (AB - Jarvis Lake)

PHYLUM NEMATHELMINTHES

CLASS NEMATODA 
CLASS ADENOPHORA

\section{ORDER ENOPLIDA}

\section{SUPERFAMILY \\ DIOCTOPHYMATOIDEA}

\section{FAMILY DIOCTOPHYMATIDAE}

*Eustrongylides iubifex (Nitzsch in

Rudolphi, 1819) Jägerskiöld, 1909

Site: mesenteries

Hosts: Catostomus catostomus (7) Catostomus commersoni $(3,6,7)$

Culaea inconstans (7)

Perca flavescens $(1,2,3,4,5,6,7)$

Stizostedion vitreum (4)

Distribution: $\mathrm{ON}$

Records: 1) Measures 1987 ; 2) Measures 1988a; 3) Measures 1988b; 4) Dechtiar \& Christie 1988; 5) Dechtiar et al. 1988; 6) Dechtiar \& Nepszy 1988; 7) Dechtiar et al. 1989

*Eustrongylides sp.

Sites: coelom, gonads, mesenteries, musculature, viscera

Hosts: Catostomus commersoni $(1,2,3$,

18)

Catostomus macrocheilus (3)

Cottus cognatus $(11,13)$

Gasterosteus aculeatus (3, 7, 9)

Lota lota $(11,12,13,14)$

Oncorhynchus clarki $(3,10)$

Oncorhynchus kisutch (4)
14)

Oncorhynchus mykiss $(10,11,13$,

Perca flavescens $(5,6,8)$

Prosopium cylindraceum (3)

Prosopium williamsoni $(3,11,12$, 13, 14, 17)

Ptychocheilus oregonensis (11,

12, 13, 14)

Salvelinus fontinalis $(15,16)$

Salvelinus malma $(3,11,13)$

Salvelinus namaycush $(3,14)$

Stizostedion canadense $(5,6)$

Stizostedion vitreum (6)

Distribution: $\quad \mathrm{BC}, \mathrm{NB}, \mathrm{ON}, \mathrm{QC}$

Records: $\quad$ 1) Bangham 1941 (ON); 2)

Bangham \& Venard 1946 (ON); 3)

Bangham \& Adams 1954 (BC); 4)

Godfrey 1968 (BC); 5) Dechtiar 1972a

(ON); 6) Dechtiar $1972 b(\mathrm{ON})$; 7) Hanek

\& Molnar 1974 (QC); 8) Molnar et al.

1974 (ON); 9) Lester 1975 (BC); 10)

Hoskins et al. $1976(\mathrm{BC}) ; 11)$ Anonymous 1978 (BC); 12) Anonymous 1981 (BC);

13) Arai \& Mudry 1983 (BC); 14)

Anonymous 1984 (BC); 15) Frimeth 1987a (NB); 16) Frimeth 1987b (NB);

17) Nener et al. 1995 (BC); 18) Couillard et al. 1995 (QC)

\section{SUPERFAMILY TRICHUROIDEA}

\section{FAMILY CAPILLARIIDAE}

Pseudocapillaria (Ichthyocapillaria) salvelini (Polyansky, 1852) Moravec, 1982

Synonym: Capillaria salvelini Polyansky, 1952 
Site: digestive tract

Hosts: Coregonus clupeaformis $(3,6,15)$

Oncorhynchus clarki (1)

Oncorhynchus kisutch $(1,8,9$ 10,

11, 14)

Oncorhynchus mykiss (15)

Prosopium cylindraceum $(3,5,15)$

Salvelinus fontinalis $(1,2,3,4,6$, $12,13,16)$

Salvelimus fontinalis X Salvelinus

namaycush (7)

Salvelinus malma (11)

Salvelinus namaycush $(3,5,6,8$, 10)

Thymallus arcticus (5)

Distribution: $\quad \mathrm{BC}, \mathrm{LB}, \mathrm{NB}, \mathrm{NF}, \mathrm{ON}, \mathrm{QC}$, YT

Records: 1) Threlfall \& Hanek 1969

(NF); 2) Threlfall \& Hanek 1970c (NF);

3) Hicks \& Threlfall 1973 (LB); 4) Hanek

\& Molnar 1974 (QC); 5) Arthur et al.

1976 (YT); 6) Chinniah \& Threlfall 1978

(LB); 7) Dechtiar \& Berst 1978 (ON); 8)

Moravec 1980 (BC, NF, ON, YT); 9)

Anonymous 1981 (BC); 10) Bell \&

Beverley-Burton 1981 (BC, NB, YT); 11)

Anonymous 1984 (BC); 12) Frimeth

1987a (NB); 13) Frimeth 1987b (NB);

14) Dechtiar \& Christie 1988 (ON); 15)

Dechtiar \& Lawrie 1988 (ON); 16)

Marcogliese \& Cone 1991b (NF)

Pseudocapillaria (Pseudocapillaria) tomentosa (Dujardin, 1843) Moravec, 1987

Synonyms: Capillaria catostomi Pearse, 1924; Hepaticola bakeri Mueller and Van Cleave, 1932 (partim); Skrjabinocapillaria bakeri (Mueller and Van Cleave, 1932) Skrjabin and
Shikhobalova, 1954

Site: intestine

Hosts: Catostomus catostomus $(1,3)$

Catostomus commersoni $(1,4,6,7$,

8)

Catostomus macrocheilus (3)

Coregonus clupeaformis (8)

Couesius plumbeus (3)

Lota lota $(1,2,5)$

Oncorhynchus clarki (3)

Oncorhynchus kisutch (3)

Oncorhynchus mykiss (3)

Prosopium williamsoni (3)

Ptychocheilus oregonensis (3)

Richardsonius balteatus (3)

Salvelinus fontinalis $(2,3)$

Salvelinus malma (3)

Distribution: $\quad \mathrm{BC}, \mathrm{ON}$

Records: 1) Bangham 1941 (ON); 2)

Bangham \& Venard 1946 (ON); 3)

Bangham \& Adams 1954 (BC); 4)

Bangham 1955 (ON); 5) Dechtiar 1972a

(ON); 6) Bell \& Beverley-Burton 1980

(ON); 7) Moravec 1980 (ON); 8) Bell \&

Beverley-Burton 1981 (ON)

Capillaria (Capillaroides) catenata Van

Cleave and Mueller, 1932

Synonym: Thominx catenata (Van

Cleave and Mueller, 1932) Skrjabin and

Shikhobalova, 1954)

Site: intestine

Host: Catostomus commersoni

Distribution: QC

Record: Fantham \& Porter 1948

Capillariidae gen. sp.

Site: [intestine] 
Hosts: Oncorhynchus mykiss

Ptychocheilus oregonensis

Distribution: $\quad \mathrm{BC}$

Record: Anonymous 1984

SUBCLASS SECERNENTEA

ORDER ASCARIDIDA

SUPERFAMILY ASCARIDOIDEA

FAMILY ANISAKIDAE

*Contracaecum spiculigerum (Rudolphi, 1809) Railliet and Henry, 1912

Sites: liver, mesenteries

Hosts: Perca flavescens $(2,3,4,5)$

Ptychocheilus oregonensis (1)

Distribution: $\quad \mathbf{A B}, \mathbf{B C}, \mathrm{ON}$

Records: 1) Bangham \& Adams 1954

(BC); 2) Tedla \& Fernando 1969a (ON);

3) Tedla 1969 (ON); 4) Tedla \& Fernando 1972 (ON); 5) Zelmer 1994 (AB -

Elkwater Lake, Fincastle Lake, Rattlesnake Lake, Sherburne Lake, Spruce Coulee Reservoir)

\section{${ }^{*}$ Contracaecum sp.}

Sites: coelom, mesenteries, musculature, viscera

Hosts: Catostomus commersoni $(4,5,6$, $14,16)$

Coregonus clupeaformis $(8,17)$

Culaea inconstans $(10,11)$
Esox lucius (14)

Etheostoma exile (5)

Gasterosteus aculeatus $(3,13)$

Moxostoma anisurum (14)

Notropis atherinoides (14)

Notropis hudsonius $(5,14)$

Oncorhynchus clarki (2)

Oncorhynchus mykiss (11)

Perca flavescens $(1,5,12,14)$

Percina caprodes (5)

Phoxinus eos (16)

Pimephales promelas $(9,10,11)$

Pungitius pungitius $(10,11,13$,

15)

Salvelinus fontinalis $(7,16)$

Salvelinus malma (2)

Stizostedion vitreum (1)

Distribution: $\quad$ AB $, \mathrm{BC}, \mathrm{LB}, \mathrm{MB}, \mathrm{NF}, \mathrm{NS}$, ON, QC

Records: 1) Worley \& Bangham 1952

(QC); 2) Bangham \& Adams 1954 (BC);

3) Hanek \& Threlfall 1970a (NF); 4)

Threlfall \& Hanek 1970a (LB); 5)

Dechtiar 1972a (ON); 6) Kakonge 1972

(ON); 7) Hicks \& Threlfall 1973 (LB); 8)

Drouin 1982 (AB - McGregor Lake); 9)

McAllister \& Mudry 1983 (AB -

Cardinal Lake); 10) Dick 1987 (MB);

11) Dick et al. 1987 (MB); 12) Szalai \&

Dick 1991a (MB); 13) Marcogliese 1992

(NS); 14) Szalai et al. 1992 (MB); 15)

Marcogliese 1995 (NS); 16) Dubois et al. 1996 (QC); 17) Hobbs (undated) (AB -

Lesser Slave Lake)

**Hysterothylacium aduncum (Rudolphi, 1802) Deardorff and Overstreet, 1981

Synonyms: Contracaecum gadi (O. F. Müller, 1777); Contracaecum clavatum (Rudolphi, 1809); Contracaecum 
aduncum Rudolphi, 1802; Thynnascaris adunca (Rudolphi, 1802) Hartwich, 1957

Sites: coelom, digestive tract, mesenteries, musculature, viscera

Hosts: Salvelinus namaycush

Distribution: NT

Records: $\quad$ Stewart \& Bernier 1983; Stewart \& Bernier 1984

*Hysterothylacium brachyurum Ward and Magath, 1917

Synonyms: Contracaecum brachyurum (Ward and Magath, 1917) Van Cleave and Mueller, 1934; Thynnascaris brachyura (Ward and Magath, 1917) Margolis and Arthur, 1979

Sites: intestine, liver, mesenteries Hosts: Culaea inconstans (7)

Lota lota $(2,4)$

Moxostoma anisurum (1) Noturus flavus (4)

Perca flavescens $(3,5,6,7)$

Salvelinus fontinalis (7)

Salvelinus fontinalis X Salvelinus

namaycush (7)

Salvelinus malma (2)

Stizostedion canadense (1)

Stizostedion vitreum (3)

Distribution: $\quad \mathrm{BC}, \mathrm{ON}$

Records: 1) Dechtiar 1972a (ON); 2)

Arai \& Mudry 1983 (BC); 3) Dechtiar \& Christie 1988 (ON); 4) Dechtiar et al. 1988 (ON); 5) Dechtiar \& Lawrie 1988 (ON); 6) Dechtiar \& Nepszy 1988 (ON);

7) Dechtiar et al. 1989 (ON)

Hysterothylacium brachyurum Ward and Magath, 1917
Synonyms: Contracaecum brachyurum (Ward and Magath, 1917) Van Cleave and Mueller, 1934; Thynnascaris brachyura (Ward and Magath, 1917) Margolis and Arthur, 1979

Sites: intestine, pyloric caeca

Hosts: Coregonus artedii $(8,12)$ Esox lucius $(2,3,5,6,8,13,14$, $16,17,18,19)$

Etheostoma exile $(2,5)$

Lota lota $(1,4,6,7,9,15,18)$

Notropis atherinoides (6)

Perca flavescens $(10,18)$

Percopsis omiscomaycus $(2,5,6$, 17)

Salvelinus fontinalis $(1,18,20)$

Salvelinus fontinalis $\mathrm{X}$ Salvelinus

namaycush $(11,17,20)$

Salvelinus malma $(9,15)$

Salvelinus namaycush (7)

Stizostedion canadense (6)

Stizostedion vitreum $(2,5,6,10$, $16,17)$

Distribution: $\quad$ AB, BC, MB, ON, QC

Records: $\quad$ 1) Bangham \& Venard 1946

(ON); 2) Bangham 1951 (ON); 3) Worley \& Bangham 1952 (QC); 4) Bangham \& Adams 1954 (BC); 5) Bangham 1955 (ON); 6) Dechtiar 1972a (ON); 7) Mudry \& Anderson 1977 (AB - Upper

Waterton Lake); 8) Watson 1977 (MB); 9) Anonymous 1978 (BC); 10) Anthony 1978b (ON); 11) Dechtiar \& Berst 1978 (ON); 12) Watson \& Dick 1979 (MB); 13) Watson \& Dick 1980 (MB); 14) Anthony 1983 (ON); 15) Arai \& Mudry 1983 (BC); 16) Dechtiar \& Christie 1988 (ON); 17) Dechtiar et al. 1988 (ON); 18) Dechtiar \& Lawrie 1988 (ON); 19) Dechtiar \& Nepszy (ON); 20) Dechtiar et al. $1989(\mathrm{ON})$ 
${ }^{* *}$ Hysterothylacium sp.

Sites: digestive tract, mesenteries

Hosts: Coregonus clupeaformis (2)

Culaea inconstans (4)

Gasterosteus aculeatus (3)

Oncorhynchus mykiss (2)

Prosopium williamsoni (2)

Ptychocheilus oregonensis (2)

Salvelinus fontinalis (1)

Salvelinus malma (2)

Distribution: $\quad \mathrm{BC}, \mathrm{ON}, \mathrm{QC}$

Records: $\quad$ 1) Black 1981 (QC); 2)

Anonymous 1984 (BC); 3) Dechtiar \&

Christie 1988 (ON); 4) Dechtiar et al.

1988 (ON)

*Raphidascaris acus (Bloch, 1779) Raillet and Henry, 1915

Synonyms: Raphidascaris canadensis

Smedley, 1933; Raphidascaris

laurentianus Richardson, 1937;

Raphidascaris alius Lyster, 1940

Site: viscera

Hosts: Acipenser fulvescens (27)

Catostomus commersoni $(9,15,16$,

$23,26,29$ )

Coregonus artedii $(9,15,16,23$, 26, 29)

Coregonus clupeaformis $(1,6,9$, $14,18)$

Cottus cognatus (1)

Etheostoma exile (16)

Hiodon alosoides $(23,26)$

Lota lota (17)

Moxostoma anisurum $(23,26)$

Notropis hudsonius $(23,26)$

Perca flavescens $(3,5,7,9,10$,

$11,12,13,16,18,19,20,21,22,23$,

$24,25,26,27,30$ )
Percina caprodes $(23,26)$

Pimephales promelas (16)

Percopsis omiscomaycus (23)

Ptychocheilus oregonensis $(2,8)$

Pungitius pungitius (4)

Salvelinus fontinalis (29)

Salvelimus namaycush (4)

Stizostedion canadense $(23,26)$

Stizostedion vitreum $(9,13,23$,

26)

Thymallus arcticus (1)

Distribution: $\quad \mathbf{A B}, \mathrm{BC}, \mathbf{M B}, \mathrm{ON}, \mathrm{QC}$, SK, YT

Records: $\quad$ 1) Arthur et al. 1976 (YT); 2) Anonymous 1978 (BC); 3) Pybus \& Samuel 1978 (AB - Cache Lake); 4)

Leong \& Holmes 1981 (AB - Cold Lake); 5) Smith \& Anderson 1981 (ON);

6) Samuel 1981 (AB - Blue Lake, Jarvis Lake); 7) Smith \& Anderson 1981 (ON);

8) Arai \& Mudry 1983 (BC); 9) Poole 1983 (MB); 10) Poole \& Dick 1984 (MB); 11) Smith 1984b (ON); 12) Baker 1984b (ON); 13) Poole \& Dick 1986 (MB); 14) Samuel 1985 (AB - Blue Lake, Jarvis Lake); 15) Shostak \& Dick 1986 (MB); 16) Smith 1986 (ON); 17) Anthony 1987 (ON); 18) Dechtiar et al. 1988 (ON); 19) Dechtiar \& Lawrie 1988 (ON); 20) Stock 1988 (AB - Jarvis Lake); 21) Dechtiar \& Nepszy 1988 (ON); 22) Dechtiar et al. 1989 (ON); 23) Szalai 1989 (MB); 24) Szalai \& Dick 1991 (MB); 25) Szalai et al. 1992a (MB); 26) Szalai et al. 1992b (MB); 27) Choudhury \& Dick 1993 (MB); 28) Zelmer 1994 (AB - Carburn Pond, Elkwater Lake, Fincastle Reservoir, Garner Lake, Pigeon Lake, Pine Lake, Rattlesnake Reservoir, Sherburne Reservoir, Sylvan Lake); 29) Dubois et al. 1996 (QC); 30) Zelmer \& Arai 1998 


\section{(AB - Garner Lake)}

Raphidascaris acus (Bloch, 1779) Railliet and Henry, 1915

Synonyms: Raphidascaris canadensis

Smedley, 1933; Raphidascaris

laurentianus Richardson, 1937;

Raphidascaris alius Lyster, 1940

Site: intestine

Hosts: Catostomus catostomus (25)

Catostomus commersoni (24)

Coregonus artedii (24)

Coregonus clupeaformis $(17,21$,

$24,25,34)$

Esox lucius (1, 4, 5, 6, 7, 8, 9, 10,

$11,13,14,15,16,17,18,19,20,21,22$, $23,24,25,26,28,29,30,31,33,35,36$, $37,39,40$ )

Hiodon alosoides $(37,40)$

Lota lota $(25,32)$

Oncorhynchus mykiss (26)

Perca flavescens $(12,21,24,27$,

$28,31,37,38,40$ )

Salvelinus fontinalis $(2,3,25,26$,

41)

Stizostedion vitreum $(6,9,10,12$,

$24,25,27,29,37,40$ )

Distribution: $\quad$ AB, LB, MB, NT, ON, QC, SK, YT

Records: 1) Smedley 1933 (MB, SK); 2)

Richardson 1937 (QC); 3) Lyster 1940a

(QC); 4) McLeod 1944 (MB); 5) Miller

1945d (AB - Lake Athabasca); 6)

Rawson 1951 (NT); 7) Stewart-Hay

$1951 b$ (MB); 8) Stewart-Hay 1951f

(MB); 9) Stewart-Hay 1952a (MB); 10)

Stewart-Hay 1952b (MB); 11) Stewart-

Hay 1953a (MB); 12) Dickson 1964

(MB); 13) Arthur et al. 1976 (YT); 14)

Chinniah \& Threlfall 1978 (LB); 15)
Gordon et al. 1978 (QC); 16) Thompson \& Threlfall 1978 (QC); 17) Pybus \&

Samuel 1978 (AB - Blue Lake, Cache

Lake, Jarvis Lake); 18) Kinnis \& Curtis 1981 (QC); 19) Leong \& Holmes 1981 (AB - Cold Lake); 20) Smith \& Anderson 1981 (ON); 21) Samuel 1981 [AB - Jarvis Lake (E. lucius), Cache Lake (P. flavescens)]; 22) Smith \& Anderson 1982 (ON); 23) Anthony 1983 (ON); 24) Poole 1983 (MB); 25) Smith 1984a (ON); 26) Smith 1984b (ON); 27) Poole \& Dick 1985 (MB); 28) Samuel 1985 [AB - Blue Lake (E. lucius), Cache Lake, Jarvis Lake (E. lucius, $P$. flavescens)]; 29) Poole \& Dick 1986 (MB); 30) Shostak \& Dick 1986 (MB); 31) Smith 1986 (ON); 32) Anthony 1987 (ON); 33) Dechtiar et al. 1988 (ON); 34) Stock 1988 (AB - Jarvis Lake); 35) Shostak \& Dick 1989 (MB); 36) Szalai \& Dick 1989 (MB); 37) Szalai 1989 (MB); 38) Szalai \& Dick 1991a (MB); 39) Szalai et al. 1992a; 40) Szalai et al. 1992b (MB); 41) Dubois et al. 1996 (QC)

**Raphidascaris sp.

Sites: digestive tract, liver, musculature Hosts: Acipenser fulvescens $(20,21)$

Coregonus artedii $(9,13)$

Coregonus clupeaformis $(9,11$, $13,15,16,17)$

Esox lucius $(2,3,6,7,8,9,12$,

14, 15, 16)

Perca flavescens $(1,5,10)$

Pungitius pungitius (8)

Salvelinus fontinalis $(11,18,19)$

Salvelinus namaycush $(8,11)$

Stizostedion vitreum $(4,10)$

Distirbution: $\quad$ AB, LB, MB, NB, ON, 
QC, SK

Records: 1)Richardson 1937 (QC); 2) McLeod 1943 (MB); 3) McLeod 1944 (MB); 4) Stewart-Hay 1951b (MB); 5) Stewart-Hay 1952a (MB); 6) StewartHay 1953a (MB); 7) Threlfall \& Hanek 1970b (LB); 8) Leong 1975 (AB - Cold Lake); 9) Watson 1977 (MB); 10) Anthony 1978b (LB); 11) Chinniah \& Threlfall 1978 (LB); 12) Pybus \&

Samuel 1978 (AB - Angling Lake); 13) Watson \& Dick 1979 (MB); 14) Watson \& Dick 1980 (MB); 15) Samuel 1981 (AB - Blue Lake, Jarvis Lake); 16) McAllister \& Mudry 1983 (AB - Lower Mann Lake, Upper Mann Lake); 17) Samuel 1985 (AB - Jarvis Lake); 18) Frimeth 1987a (NB); 19) Frimeth 1987b (NB); 20) Choudhury et al. 1990 (MB); 21) Choudhury \& Dick 1993 (MB, SK)

*Anisakidae gen. sp.

Sites: mesenteries, peritoneum Host: Prosopium williamsoni Distribution: $\quad \mathrm{BC}$

Record: Anonymous 1984

SUPERFAMILY SEURATOIDEA

FAMILY CUCULLANIDAE

Cucullanellus cotylophora (Ward and Magath, 1917) Petter, 1974

Synonyms: Dichelyne cotylophora (Ward and Magath, 1917); Dacnitoides cotylophora Ward and Magath, 1917
Site: intestine

Hosts: Lota lota $(11,15)$

Perca flavescens $(1,2,3,4,5,6,7$, $8,9,10,11,12,13,14,16,17,18,19$, 20)

Stizostedion vitreum $(2,5,7,16)$

Distribution: ON, QC

Records: 1) Smedley 1933 (ON); 2)

Bangham \& Hunter 1939 (ON); 3)

Bangham 1941 (ON); 4) Bangham \& Venard 1946 (ON); 5) Bangham 1951 (ON); 6) Worley \& Bangham 1952 (QC); 7) Bangham 1955 (ON); 8) Tedla \& Fernando 1969a (ON); 9) Tedla 1969 (ON); 10) Tedla \& Fernando 1972 (ON); 11) Dechtiar 1972a (ON); 12) Cannon 1973 (ON); 13) Baker 1984a (ON); 14) Baker 1984b (ON); 15) Anthony 1987 (ON); 16) Dechtiar \& Christie 1988 (ON); 17) Dechtiar et al. 1988 (ON); 18) Dechtiar \& Lawrie 1988 (ON); 19) Dechtiar \& Nepszy 1988 (ON); 20) Choudhury \& Dick 1996c (MB)

\section{Cucullanus sp.}

Site: intestine Host: Perca flavescens Distribution: ON Record: Anthony $1978 \mathrm{~b}$

Truttaedacnitis clitellarius (Ward and Magath, 1917) Petter, 1974

Synonym: Cucullanus clitellarius Ward and Magath, 1917

Site: intestine

Host: Acipenser fulvescens

Distribution: $\quad \mathrm{CA}, \mathrm{MB}, \mathrm{ON}, \mathrm{SK}$

Records: Bangham \& Hunter 1939 
(ON); Bangham 1951 (ON); Bangham 1955 (ON); Anthony 1974 (ON);

Dechtiar Christie 1988 (ON); Dechtiar \&

Lawrie 1988 (ON); Choudhury et al. 1990 (MB, SK); Swanson et al. 1991 (MB);

Choudhury \& Dick 1993 (MB, SK);

Choudhury \& Dick 1996a (MB, SK);

Choudhury \& Dick 1996c (CA)

Truttaedacnitis truttae (Fabricius, 1794)

Petter, 1974

Synonyms: Bulbodacnitis truttae

(Fabricius, 1794) Maggenti, 1971;

Bulbodacnitis alpinus Mudry and

McCart, 1974

Sites: intestine, pyloric caeca

Hosts: Coregonus clupeaformis (10)

Oncorhynchus clarki (2)

Oncorhynchus mykiss $(1,2,3,4$,

$5,6,7,8,9,10,14)$

Prosopium cylindraceum (2)

Prosopium williamsoni $(2,4,5,7$,

$8,10,13)$

Ptychocheilus oregonensis $(7,10)$

14)

Salvelinus fontinalis $(2,11,12$,

14)

Salvelinus malma $(2,5,7,8,10$,

14)

Salvelinus namaycush $(4,5,8,10$,

Distribution: $\quad \mathbf{A B}, \mathrm{BC}, \mathrm{NB}$

Records: 1) Smedley 1933 (BC); 2)

Bangham \& Adams 1954 (BC); 3) Mudry

\& McCart 1974 (BC); 4) Mudry \&

Anderson 1977 [AB - Lower Waterton

Lake ( $P$. williamsoni), Pyramid Lake

(O. mykiss, $P$. williamsoni, $S$.

namaycush)]; 5) Anonymous 1978 (BC);

6) Russell 1980 (BC); 7) Anonymous

1981 (BC); 8) Arai \& Mudry 1983 (BC);
9) Dunn et al. 1983 (BC); 10)

Anonymous 1984 (BC); 11) Frimeth

1987a (NB); 12) Frimeth 1987b (NB);

13) Nener et al. 1995 (BC); 14)

Choudhury \& Dick 1996c (BC, NB)

Truttaedacnitis sp.

Synonym: Bulbodacnitis sp.

Site: not specified

Hosts: Rhinichthys cataractae

Richardsonius balteatus

Salvelinus fontinalis

Distribution: $\quad$ BC

Record: Bangham \& Adams 1954

FAMILY QUIMPERIDAE

Haplonema hamulatum Moulton, 1931

Synonyms: Ichtyobronema conoura (v. Linstow, 1885) sensu Gnedina and Savina (1930); Ichtyobronema gnedini Sudarikov and Ryzhikov, 1952;

Cottocomephoronema problematica

Layman, 1933; C. hamulatum (Moulton,

1931) Sudarikov and Ryzhikov, 1952

Site: intestine

Hosts: Lota lota (1, 2, 3, 4, 5, 6, 7, 8, 9, $10,11,12,13,14,15)$

Oncorhynchus mykiss $(9,12)$

Prosopium williamsoni $(9,12)$

Salvelinus malma $(9,12)$

Distribution: $\quad \mathbf{A B}, \mathrm{BC}, \mathrm{ON}, \mathrm{YT}$

Records: 1) Moulton 1931 (ON); 2)

Bangham \& Hunter 1939 (ON); 3)

Bangham 1941 (ON); 4) Bangham \& Venard 1946 (ON); 5) Bangham 1951 (ON); 6) Bangham 1955 (ON); 7) Arthur 
\& Margolis 1975 (YT); 8) Arthur et al.

1916

1976 (YT); 9) Anonymous 1978 (BC);

10) Anonymous 1981 (BC); 11) Leong \&

Holmes 1981 (AB - Cold Lake); 12)

Arai \& Mudry 1983 (BC); 13)

Anonymous 1984 (BC); 14) Dechtiar et

al. 1988 (ON); 15) Dechtiar \& Lawrie

$1988(\mathrm{ON})$

Haplonema sp.

Site: intestine

Hosts: Cottus cognatus (1)

Lota lota $(2,3)$

Distribution: $\quad \mathbf{A B}, \mathbf{B C}, \mathrm{LB}$

Records: 1) Bangham \& Adams 1954

(BC); 2) Threlfall \& Hanek 1971 (LB); 3)

Leong 1975 (AB - Cold Lake)

*Seuratinae gen. sp.

Site: [digestive tract]

Host: Salvelinus fontinalis

Distribution: QC

Record: Black 1981a

ORDER SPIRURIDA

SUBORDER CAMALLANINA

SUPERFAMILY CAMALLANOIDEA

FAMILY CAMALLANIDAE

Site: [intestine]

Hosts: Esox lucius (2)

Perca flavescens $(1,3)$

Camallanus ancylodirus Ward and Magath,

Site: intestine

Host: Carpiodes cyprinus

Distribution: ON

Record: Dechtiar \& Nepszy 1988

* *Camallanus oxycephalus Ward and Magath, 1917

Site: intestine

Hosts: Carpiodes cyprinus $(3,7)$

Catostomus commersoni $(4,5)$

Culaea inconstans (4)

Esox lucius (6)

Etheostoma exile $(4,6)$

Hiodon tergisus (1)

Lota lota (4)

Notropis atherinoides (1)

Notropis hudsonius (1)

Noturus flavus (1)

Oncorhynchus kisutch (6)

Perca flavescens $(1,4,7)$

Percina caprodes $(1,4,6)$

Percopsis omiscomaycus (1)

Rhinichthys cataractae (1)

Stizostedion canadense $(1,4)$

Stizostedion vitreum $(1,7)$

Distribution: ON

Records: 1) Bangham \& Hunter 1939; 2)

Bangham 1955; 3) Dechtiar 1972b; 4)

Dechtiar 1972a; 5) Chan 1980; 6)

Dechtiar \& Christie 1988; 7) Dechtiar \&

Nepszy 1988

\section{Camallanus sp.}


Distribution: LB, ON

Records: 1) Tedla 1969 (ON); 2)

Threlfall \& Hanek 1970b (LB); 3) Tedla

\& Fernando 1972 (ON)

\section{SUPERFAMILY DRACUNCULOIDEA}

\section{FAMILY PHILOMETRIDAE}

**Philometra cylindracea (Ward and Magath, 1917) Van Cieave and Mueller, 1934

Site: coelom

Hosts: Perca flavescens $(1,2,3,4,5,6,7$, $8,9,10,11,12,13$ )

Salvelinus fontinalis (2)

Distribution: ON, QC

Records: 1) Bangham \& Hunter 1939

(ON); 2) Fantham \& Porter 1948 (QC);

3) Bangham 1951 (ON); 4) Bangham 1955 (ON); 5) Tedla 1969 (ON); 6) Tedla \& Fernando 1969a (ON); 7) Tedla \& Fernando 1972 (ON); 8) Dechtiar 1972a (ON); 9) Molnar et al. 1974 (ON); 10) Molnar \& Fernando 1975a (ON); 11) Dechtiar \& Christie 1988 (ON); 12) Dechtiar et al. $1988(\mathrm{ON})$; 13) Dechtiar \& Nepszy 1988 (ON)

Philometra kobuleji Molnar and Fernando, 1975

Sites: coelom, mesenteries, swin bladder Host: Catostomus commersoni

Distribution: ON

Records: Molnar \& Fernando 1975b; Chan 1980; Molnar et al. 1982
**Philometra sp.

Sites: circulatory system, coelom, eyes, gills, intestine

Hosts: Catostomus catostomus (5)

Catostomus commersoni $(6,7,8)$

Coregonus artedii $(2,3)$

Coregonus clupeaformis $(2,3)$

Perca flavescens $(1,4)$

Prosopium cylindraceum $(2,3,7)$

Salvelinus fontinalis (1)

Distribution: ON, QC

Records: $\quad$ 1) Fantham \& Porter 1948

(QC); 2) Bangham 1951 (ON); 3)

Bangham 1955 (ON); 4) Dechtiar 1972a

(ON); 5) Hanek \& Molnar 1974 (QC); 6)

Molnar et al. 1974 (ON); 7) Mackie et al.

1983 (ON); 8) Dechtiar et al. 1989 (ON)

Philometroides huronensis Uhazy, 1976

Sites: fins, swim bladder

Hosts: Catostomus catostomus $(4,6,10)$ Catostomus commersoni $(1,2,3$,

$4,5,6,7,8,9,10,11)$

Catostomus macrocheilus $(6,10)$

Moxostoma macrolepidotum (4)

Distribution: $\quad \mathrm{BC}, \mathrm{ON}$

Records: 1) Uhazy 1976 (ON); 2) Uhazy \& Anderson 1976 (ON); 3) Uhazy 1977a (ON); 4) Uhazy 1977b (ON); 5) Uhazy 1978 (ON); 6) Anonymous 1978 (BC); 7) Chan 1980 (ON); 8) Molnar et al. 1982 (ON); 9) Arai \& Mudry 1983 (BC); 10) Mackie et al. 1983 (ON); 11) Dubois et al.1996 (QC)

Philometroides nodulosa (Thomas, 1929) Dailey, 1967 
Synonym: Philometra nodulosa Thomas, 1929

Sites: cheek galleries, subcutaneous fissures

Hosts: Carpiodes cyprinus $(2,7)$ Catostomus catostomus (6) Catostomus commersoni $(1,3,4$, $5,6,7,8,9,10,11)$

Distribution: $\mathrm{MB}, \mathrm{ON}, \mathrm{QC}$

Records: 1) Fantham \& Porter 1948 (QC); 2) Dechtiar 1972a (ON); 3) Chan 1980 (ON); 4) Molnar et al. 1982 (ON);

5) Dechtiar \& Christie 1988 (ON); 6)

Dechtiar et al. 1988 (ON); 7) Dechtiar \& Nepszy 1988 (ON); 8) Dechtiar et al. 1989 (ON); 9) Szalai 1989 (MB); 10)

Szalai et al. 1992 (MB); 11) Dubois et al. 1996 (QC)

*Philometroides sp.

Site: eye

Host: Catostomus commersoni

Distribution: ON

Record: Chan 1980

Philonema agubernaculum Simon and

Simon, 1936

Sites: coelom, swim bladder

Hosts: Coregonus artedii $(6,7,10)$

Coregonus clupeaformis $(4,6,14)$

Gasterosteus aculeatus (5)

Oncorhynchus kisutch $(6,7,9,10$,

19)

Oncorhynchus mykiss $(3,7,8,9$,

$10,11,13,14,16,17,19)$

Prosopium cylindraceum (4)

Prosopium williamsoni $(7,8,9$,

$10,11,14,18)$
16)

Salvelinus fontinalis $(1,2,4,5$,

Salvelinus malma $(10,14)$

$14,15)$

Salvelinus namaycush $(4,6,7,12$,

Distribution: $\quad$ AB, BC, EXP, LB, NF, NT, QC

Records: 1) Richardson 1936 (QC); 2)

Sandeman \& Pippy 1967 (NF); 3) Ko \& Anderson 1969 (BC); 4) Hicks \& Threlfall 1973 (LB); 5) Hanek \& Molnar 1974 (QC); 6) Leong \& Holmes 1974a (AB - Cold Lake); 7) Leong 1975 (AB Cold Lake); 8) Anonymous 1978 (BC); 9) Anonymous 1981 (BC); 10) Leong \& Holmes 1981 (AB - Cold Lake); 11) Arai \& Mudry 1983 (BC); 12) Stewart \& Bernier 1983 (NT); 13) Bashirullah 1983 (EXP); 14) Anonymous 1984 (BC); 15) Stewart \& Bernier 1984 (NT); 16) Marcogliese \& Cone 1991b (NF); 17) Adamson et al. 1992 (BC); 18) Nener et al. 1995 (BC); 19) Despres et al. 1995 (BC)

**Philonema oncorhynchi KuitunenEkbaum, 1933

Sites: coelom, swim bladder

Hosts: Coregonus artedii (1) Coregonus clupeaformis (1) Oncorhynchus clarki $(1,2)$ Oncorhynchus kisutch (2) Oncorhynchus mykiss $(2,3,4)$ Salvelinus fontinalis (1) Salvelinus malma (2) Salvelinus namaycush (1)

Distribution: $\quad$ BC, EXP, NS, ON

Records: 1) Kuitunen-Ekbaum $1937 \mathrm{~b}$

(BC, NS, ON); 2) Bangham \& Adams 1954 (BC); 3) Bell \& Hoskins 1971 (BC); 
4) Bashirullah 1983 (EXP)

**Philonema sp.

Site: coelom

Hosts: Catostomus commersoni (11)

Coregonus artedii $(2,5,7,12)$

Coregonus clupeaformis $(9,10)$

Esox lucius (5)

Oncorhynchus clarki (8)

Oncorhynchus mykiss $(9,10)$

Prosopium cylindraceum (6)

Prosopium williamsoni $(6,9,10)$

Salveïinus fontinalis (4)

Salvelinus malma $(9,10)$

Salvelinus namaycush $(1,2,3,5$,

10)

Thymallus arcticus $(9,10)$

Distribution: $\quad$ AB, BC, LB, MB, NT, ON

Records: 1) MacLulich 1943b (ON); 2)

Miller 1945d (AB - Lake Athabasca ( $S$. namaycush), NT (C. artedii); 3) Miller \&

Kennedy 1948 (NT); 4) Munroe 1949

(LB); 5) Rawson 1951 (NT); 6) Bangham

\& Adams 1954 (BC); 7) Paetz \& Nelson 1970 (AB - unspecified); 8) Hoskins et al.1976 (BC); 9) Anonymous 1978 (BC);

10) Arai \& Mudry 1983 (BC); 11) Poole 1983 (MB); 12) Nelson \& Paetz 1992

(AB - unspecified)

\section{SUPERFAMILY GNATHOSTOMATOIDEA}

\section{FAMILY GNATHOSTOMATIDAE}

*Spiroxys contortus (Rudolphi, 1819)

Schneider, 1866
Site: mesenteries

Host: Perca flavescens

Distribution: ON

Record: $\quad$ Molnar et al. 1974

*Spiroxys sp.

Sites: digestive tract, mesenteries, viscera

Hosts: Catostomus commersoni (4)

Catostomus macrocheilus (1)

Culaea inconstans (7)

Perca flavescens $(2,3,5)$

Phoxinus neogaeus (7)

Ptychocheilus oregonensis (1)

Rhinichthys cataractae (6)

Richardsonius balteatus (1)

Distribution: BC, ON

Records: 1) Bangham \& Adams 1954

(BC); 2) Bangham 1951 (ON); 3)

Bangham 1955 (ON); 4) Dechtiar 1972a

(ON); 5) Baker 1984b (ON); 6) Dechtiar

\& Lawrie1988 (ON); 7) Dechtiar et al.

1989 (ON)

SUBORDER SPIRURINA

SUPERFAMILY HABRONEMATOIDEA

FAMILY CYSTIDICOLIDAE

Cystidicola farionis Fischer, 1798

Synonym: Cystidicola canadensis

Skinker, 1930

Includes: Ancyracanthus cystidicola of Wright (1879); Cystidicola stigmatura of 
Skinker (1931) and of Ko and Anderson (1960)

Sites: coelom, mesenteries, swim bladder

Hosts: Catostomus catostomus (25)

Coregonus artedii $(2,4,5,6,14$, $20,21,23,24,32,34,42,44,45$ )

Coregonus clupeaformis $(3,4,5$, $6,10,11,12,14,17,20,21,22,23,24$, $25,29,30,31,32,34,37,38,40,41,42$, $43,44,45,46,47$ )

Coregonus sp.(19)

Oncorhynchus kisutch $(23,24,32$, $34,44,45)$

Oncorhyncinus mykiss $(13,26,31$, $33,37,40,44,45)$

Prosopium cylindraceum $(13,14$, $15,25,32,37,44,45)$

Salmo trutta (32)

Salvelinus fontinalis $(7,8,20$,

32)

Salvelinus fontinalis X Salvelinus namaycush $(22,28,32,44)$

$37,40)$

Salvelinus malma $(20,27,33,36$,

Salvelinus namaycush $(1,7,8,9$, $10,11,16,18,20,23,25,26,27,32,36$, $39,40)$

Thymallus arcticus (25)

Distribution: $\quad$ AB $, \mathrm{BC}, \mathrm{EXP}, \mathrm{MB}, \mathrm{NT}$, ON, QC, SK, YT

Records: 1) Wright 1879 (ON); 2) Skinker 1930 (ON); 3) Smedley 1933

(AB - Primrose Lake; MB, ON, SK); 4) Hunter \& Bangham 1933 (ON); 5)

Ekbaum 1936 (MB, ON); 6) Bangham \& Hunter 1939 (ON); 7) Richardson 1942 (QC); 8) MacLulich 1943b (ON); 9) Miller \& Kennedy 1948 (NT); 10) Rawson 1951 (NT); 11) Stewart-Hay 1953a (MB); 12) Stewart-Hay 1953b (MB); 13) Bangham \& Adams 1954
(BC); 14) Bangham 1955 (ON); 15) Rawson 1959 (SK); 16) Rawson 1961 (SK); 17) Boyes \& Anderson 1961 (ON); 18) Dickson 1964 (MB); 19) Margolis 1967 (ON); 20) Ko \& Anderson 1969 [AB - Lake Wabamum (C. artedii), BC (S. malma, $S$. namaycush), NT, (C. clupeaformis, $S$. namaycush), ON (C. artedii, $C$. clupeaformis, $S$. fontinalis), SK ( $C$. clupeaformis, S. namaycush)]; 21) Dechtiar 1972a (ON); 22) Collins \& Dechtiar 1974 (ON); 23) Leong \& Holmes 1974a (AB - Cold Lake); 24) Leong 1975 (AB - Cold Lake); 25) Arthur et al. 1976 (YT); 26) Mudry \& Anderson 1977 [AB - Pyramid Lake (O. mykiss, $P$. williamsoni, $S$. namaycush), Patricia Lake (O. mykiss)]; 27) Anonymous 1978 (BC); 28) Dechtiar \& Berst 1978 (ON); 29) Pybus and Samuel 1978 (AB - Blue Lake, Cache Lake, Cold Lake, Gregg Lake, Jarvis Lake, Wabamum Lake); 30) Watson \& Dick 1979 (MB); 31) Black \& Lankester 1980 (EXP, ON); 32) Lankester \& Smith 1980 (ON); 33) Anonymous 1981 (BC); 34) Leong \& Holmes 1981 (AB - Cold Lake); 35) Samuel 1981 (AB - Blue Lake, Cache Lake, Gregg Lake, Jarvis Lake); 36) Arai \& Mudry 1983 (BC); 37) Black 1983a [BC (O. mykiss, P. cylindraceum, S. malma, $S$. namaycush),

Cystidicola farionis Fischer, 1798

Synonym: Cystidicola canadensis Skinker, 1930

Includes: Ancyracanthus cystidicola of Wright (1879); Cystidicola stigmatura of 
Skinker (1931) and of Ko and Anderson (1969)

Sites: coelom, mesenteries, swim bladder

Hosts: Catostomus catostomus (25)

Coregonus artedii $(2,4,5,6,14$,

$20,21,23,24,32,34,42,44,45)$

Coregonus clupeaformis $(3,4,5$,

$6,10,11,12,14,17,20,21,22,23,24$,

$25,29,30,31,32,34,37,38,40,41$,

$42,43,44,45,46,47)$

Coregonus sp. (19)

Oncorhynchus kisutch $(23,24,32$,

$34,44,45)$

Oncorhynchus mykiss $(13,26,31$,

$33,37,40,44,45$ )

Prosopium cylindraceum $(13,14$,

$15,25,32,37,44,45$ )

Prosopium williamsoni $(13,26$,

$27,33,36,40$ )

Salmo trutta (32)

Salvelinus fontinalis $(7,8,20,32)$

Salvelinus fontinalis X Salvelinus

namaycush $(22,28,32,44)$

$37,40)$

Salvelinus malma $(20,27,33,36$,

Salvelinus namaycush $(1,7,8,9$,

$10,11,16,18,20,23,25,26,27,32,36$, 39,40 )

Thymallus arcticus (25)

Distribution: $\quad \mathbf{A B}, \mathrm{BC}, \mathrm{EXP}, \mathrm{MB}, \mathrm{NT}$, ON, QC, SK, YT

Records: 1) Wright $1879(\mathrm{ON}) ; 2$ )

Skinker 1930 (ON); 3) Smedley 1933

(AB - Primrose Lake; MB, ON, SK); 4)

Hunter \& Bangham 1933 (ON); 5)

Ekbaum 1936 (MB, ON); 6) Bangham \&

Hunter 1939 (ON); 7) Richardson 1942

(QC); 8) MacLulich 1943b (ON); 9)

Miller \& Kennedy 1948 (NT); 10)

Rawson 1951 (NT); 11) Stewart-Hay

1953a (MB); 12) Stewart-Hay 1953b

(MB); 13) Bangham \& Adams 1954
(BC); 14) Bangham 1955 (ON); 15)

Rawson 1959 (SK); 16) Rawson 1961 (SK); 17) Boyes \& Anderson 1961 (ON); 18) Dickson 1964 (MB); 19) Margolis 1967 (ON); 20) Ko \& Anderson 1969 [AB - Lake Wabamum (C. artedi), BC (S. malma, S. namaycush), NT (C. clupeaformis, $S$. namaycush), ON ( $C$. artedii, $C$. clupeaformis, $S$. fontinalis), SK (C. clupeaformis, S. namaycush)]; 21) Dechtiar 1972a (ON); 22) Collins \& Dechtiar 1974 (ON); 23) Leong \& Holmes 1974a (AB - Cold Lake); 24) Leong 1975 (AB - Cold Lake); 25) Arthur et al. 1976 (YT); 26) Mudry \& Anderson 1977 [AB - Pyramid Lake (O. mykiss, $P$. williamsoni, $S$. namaycush), Patricia Lake ( $O$. mykiss)]; 27) Anonymous 1978 (BC); 28) Dechtiar \& Berst 1978 (ON); 29) Pybus \& Samuel 1978 (AB - Blue Lake, Cache Lake, Cold Lake, Gregg Lake, Jarvis Lake, Wabamum Lake); 30) Watson \& Dick 1979 (MB); 31) Black \& Lankester 1980 (EXP, ON); 32)

Lankester \& Smith 1980 (ON); 33) Anonymous 1981 (BC); 34) Leong \& Holmes 1981 (AB - Cold Lake); 35) Samuel 1981 (AB - Blue Lake, Cache Lake, Gregg Lake, Jarvis Lake); 36) Arai \& Mudry 1983 (BC); 37) Black 1983a [BC (O. mykiss, P. cylindraceum, $S$. malma, S. namaycush), NT ( $S$. namaycush), QC (S. namaycush), YT ( $S$. namaycush)]; 38) McAllister \& Mudry 1983 (AB - Pigeon Lake, Wabamum Lake); 39) Stewart \& Bernier 1983 (NT); 40) Anonymous 1984 (BC); 41) Samuel 1985 (AB - Blue Lake, Cache Lake, Gregg Lake, Jarvis Lake); 42) Dextrase \& Lankester 1987 (ON); 43) Dechtiar \& Christie 1988 (ON); 44) Dechtiar et al. 
1988 (ON); 45) Dechtiar \& Lawrie 1988 (ON); 46) Stock 1988 (AB - Jarvis Lake); 47) Hobbs (undated) (AB Lesser Slave Lake)

Cystidicola serrata (Wright, 1879) Railliet, 1916 sp. inq.

Synonyms: Ancyracanthus serratus Wright, 1879; Rhabdochona serrata (Wright, 1879)

Site: heart

Host: Coregonus artedii (or Coregonus clupeaformis)

Distribution: ON

Record: Wright 1879

Cystidicola stigmatura (Leidy, 1886) Ward and Magath, 1917

Synonym: Cystidicola cristivomeri White, 1941

Includes: Cystidicola sp. of White (1940)

Site: $\quad$ swim bladder

Hosts: Coregonus artedii $(1,8,11,17)$ Coregonus clupeaformis $(6,8,9$, $11,17,19,21,24,36,38)$ Myoxocephalus thompsoni (27) Oncorhynchus mykiss $(10,20)$ Prosopium cylindraceum $(8,10$, $11,12)$

Prosopium williamsoni $(10,20)$

Salvelinus fontinalis $(5,17,25)$

Salvelinus malma (17)

Salvelinus fontinalis X Salvelinus namaycush $(19,21)$

Salvelinus namaycush $(2,3,4,5,6$, $7,9,16,17,18,20,22,23,24,25,27$, $28,29,30,31,32,33,34,35,37$ )
Distribution: $\quad$ AB, BC, NT, ON, QC, SK Records: 1) Skinker 1930 (ON); 2) White 1941 (ON); 3) White \& Cable 1942 (ON); 4) Richardson 1942 (QC); 5) MacLulich 1943b (ON); 6) Miller 1945d (AB - Lake Athabasca, NT); 7) Miller \& Kennedy 1948 (NT); 8) Bangham 1951 (ON); 9) Stewart-Hay 1953a (MB); 10) Bangham \& Adams 1954 (BC); 11) Bangham 1955 (ON); 12) Rawson 1959 (SK); 13) Boyes \& Anderson 1961 (ON): 14) Rawson 1961 (SK); 15) Dickson 1964 (MB); 16) Margolis 1967 (ON); 17) Ko \& Anderson 1969 [BC (S. malma, $S$. namaycush); NT, SK (C. clupeaformis, $S$. namaycush), ON (C. artedii, $C$. clupeaformis, S. fontinalis)]; 18) Dechtiar 1972a (ON); 19) Collins \& Dechtiar 1974 (ON); 20) Mudry \& Anderson 1977 [AB - Pyramid Lake (O. mykiss, $P$. williamsoni, S. namaycush), Patricia Lake (O. mykiss)]; 21) Dechtiar \& Berst 1978 (ON); 22) Smith \& Lankester 1979 (ON); 23) Black \& Lankester 1980 (ON); (24) Lankester \& Smith 1980 (ON); 25) Black 1981b (ON); 26) Black \& Anderson 1981b (ON); 27) Black \& Lankester 1981a (ON); 28) Stewart \& Bernier 1982 (NT); 29) Black 1983b (ON); 30) Black 1983c (NT, ON, QC, SK); 31) Stewart \& Bernier 1983 (NT); 32) Black 1984a (ON); 33) Black 1984b (ON); 34) Stewart \& Bernier 1984 (NT); 35) Samuel 1985 (AB - Jarvis Lake); 36) Dechtiar \& Lawrie 1988 (ON); 37) Hobbs (undated) (AB - Lesser Slave Lake)

Cystidicola sp.

Sites: coelom, swim bladder 
Hosts: Coregonus artedii $(1,5,8,11)$

Coregonus clupeaformis $(3,4,5$,

11)

Esox lucius $(9,10)$

Oncorhynchus mykiss $(4,6)$

Prosopium coulteri (7)

Prosopium williamsoni $(5,11)$

Salvelinus malma (4)

Salvelinus namaycush $(2,3,4,7)$

Distribution: $\quad$ AB, BC, MB, NT, ON

Records: 1) Pritchard 1931 (ON); 2)

White 1940 (ON); 3) Stewart-Hay 1953b

(MB); 4) Ko \& Anderson 1969 (BC,

ON); 5) Paetz \& Nelson 1970 (AB - not

specified); 6) Hoskins et al. 1976 (BC);

7) Mudry \& Anderson 1977 (AB -

Upper Waterton Lake (S. namaycush); BC (P. Coulteri); 8) Anthony 1978 (ON);

9) Samuel 1981 (AB - Jarvis Lake); 10)

Samuel 1985 (AB - Jarvis Lake); 11)

Nelson \& Paetz 1992 (AB - not specified)

Metabronema sp.

Site: not specified

Host: Thymallus arcticus

Distribution: $\quad \mathrm{BC}$

Record: Bangham \& Adams 1954

Salvelinema salmonicola (Ishii, 1916)

Margolis, 1966

Site: swim bladder

Hosts: Oncorhynchus kisutch (1)

Salvelinus malma $(2,3)$

Distribution: $\quad$ BC

Records: 1) Margolis 1966; 2) Margolis

1967; 3) Margolis \& Kabata 1967
Salvelinema walkeri (Ekbaum, 1935)

Margolis, 1967

Synonym: Cystidicola walkeri Ekbaum, 1935

Site: swim bladder

Hosts: Oncorhynchus clarki (8)

$6,7,8)$

Oncorhynchus kisutch $(1,2,3,4,5$,

Distribution: $\quad \mathrm{BC}$

Records: 1) Ekbaum 1935; 2) Ekbaum 1936; 3) Margolis 1965; 4) Margolis 1967a; 5) Margolis 1967b; 6) Margolis \& Kabata 1967; 7) Godfrey 1968; 8)

Margolis \& Moravec 1982

Spinitectus acipenseri Choudhury and Dick, 1992

Site: stomach

Host: Acipenser fulvescens

Distribution: MB, SK

Records: 1) Choudhury et al. 1990;

2)Choudhury \& Dick 1991; Swanson et al.1991; 3) Choudhury \& Dick 1992; 4)

Choudhury \& Dick 1993

Spinitectus carolini Holl, 1928

Site: intestine

Hosts: Esox lucius

Perca flavescens

Distribution: ON

Record: Dechtiar \& Lawrie 1988

* *Spinitectus gracilis Ward and Magath, 1917

Site: intestine 
Hosts: Acipenser fulvescens $(15,16,17$, 21, 22)

Catostomus commersoni (13)

Coregonus artedii $(6,10,11,13$, 20, 24)

Coregonus clupeaformis $(2,3,4,5$, $8,9,10,11,13$ )

Cottus cognatus (19)

Esox lucius (1, 10, 12, 13, 20, 24)

Lota lota $(4,5,6,16)$

Moxostoma anisurum (6)

Notropis hudsonius $(4,5)$

Noturus flavus (1)

Perca flavescens $(3,4,5,6,7,8,9$,

$13,14,15,20,22,23,24)$

Percina caprodes (24)

Percopsis omiscomaycus (4, 5, 6,

$8,15,17,20,24$ )

Prosopium cylindraceum $(3,17)$

Salvelinus fontinalis X Salvelinus

namaycush $(8,9,16)$

Stizostedion vitreum $(1,13,18)$

Distribution: $\quad \mathrm{MB}, \mathrm{ON}, \mathrm{SK}$

Records: 1) Bangham \& Hunter 1939

(ON); 2) Bangham 1941 (ON); 3)

Bangham \& Venard 1946 (ON); 4)

Bangham 1951 (ON); 5) Bangham 1955

(ON); 6) Dechtiar 1972a (ON); 7)

Cannon 1973 (ON); 8) Collins \& Dechtiar 1974 (ON); 9) Dechtiar \& Berst 1978

(ON); 10) Watson 1977 (MB); 11)

Watson \& Dick 1979 (MB); 12) Watson

\& Dick 1980 (MB); 13) Poole 1983

(MB); 14) Poole \& Dick 1985 (MB); 15)

Dechtiar \& Christie 1988 (ON); 16)

Dechtiar et al. 1988 (ON); 17) Dechtiar \&

Lawrie 1988 (ON); 18) Dechtiar \&

Nepszy 1988 (ON); 19) Dechtiar et al.

1989 (ON); 20) Szalai 1989 (MB); 21)

Choudhury et al. 1990 (MB, SK); 22)

Choudhury et al. 1991a (MB); 23) Szalai

\& Dick 1991a (MB); 24) Szalai et al.
1992 (MB)

Spinitectus sp.

Site: digestive tract

Hosts: Acipenser fulvescens $(3,7,8,9)$

Perca flavescens $(1,2,4,5,6)$

Stizostedion vitreum $(2,3,4)$

Distribution: $\quad \mathrm{MB}, \mathrm{ON}, \mathrm{SK}$

Records: 1) Bangham 1941 (ON); 2)

Bangham 1951 (ON); 3) Bangham 1955

(ON); 4) Dickson 1964 (MB); 5) Tedla

1969 (ON); 6) Tedla \& Fernando 1972

(ON); 7) Anthony 1974 (ON); 8) Anthony

1977 (ON); 9) Choudhury \& Dick 1991b

(MB)

Sterliadochona ephemeridarum (v. Linstow, 1872) Petter, 1984

Synonyms: Cystidicoloides harwoodi

Chandler, 1931; Metabronema canadense

Skinker, 1931; Metabronema salvelini

(Fujita, 1922) Bayliss, 1935;

Sterliadochona tenuissima (Zeder, 1800)

Spassky and Roytman, 1957;

Cystidicoloides tenuissima (Zeder, 1800)

Rasheed, 1965; Cystidicoloides

ephemeridarum (v. Linstow, 1872)

Moravec, 1981

Site: digestive tract

Hosts: Coregonus artedii (31)

Coregonus clupeaformis $(13,24$,

$29,30,31)$

Hiodon tergisus (20)

Oncorhynchus clarki (10)

Oncorhynchus kisutch $(30,31)$

Oncorhynchus mykiss $(9,10,17$,

21, 23, 24, 30)

Prosopium cylindraceum (10) 
Prosopium williamsoni (10)

Rhinichthys cataractae (10)

Salmo trutta $(12,30)$

Salvelinus fontinalis $(1,2,3,4,5$,

$6,7,8,9,10,11,12,13,14,15,16$,

$18,19,22,25,26,27,28,32,33,34)$

Salvelinus malma $(10,17,23)$

Salvelinus namaycush $(13,31)$

Thymallus arcticus $(17,23)$

Distribution: $\quad$ BC, LB, MB, NB, NF, NS, ON, QC

Records: 1) Skinker 1931 (QC); 2)

Bangham \& Hunter 1939 (ON); 3) Lyster 1940a (QC); 4) Richardson 1942 (QC); 5)

Choquette 1948a (QC); 6) Choquette 1948b (QC); 7) Choquette 1951b (QC);

8) Choquette 1951c (QC); 9) Bangham 1951 (ON); 10) Bangham \& Adams 1954 (BC); 11) Choquette 1955 (QC); 12)

Sandeman \& Pippy 1967 (NF); 13) Hicks \& Threlfall 1973 (LB); 14) Hanek \& Molnar 1974(QC); 15) Hare \& Frantsi 1974 (NS); 16) Frantsi et al. 1975 (NS);

17) Anonymous 1978 (BC); 18) Chinniah \& Threlfall 1978 (LB); 19) Thompson \& Threlfall 1978 (QC); 20) Glenn 1980 (MB); 21) Anonymous 1981 (BC); 22) Black 1981 (QC); 23) Arai \& Mudry 1983 (BC); 24) Anonymous 1984 (BC); 25) Cone \& Ryan 1984 (NF); 26) Frimeth 1987a (NB); 27) Frimeth 1987b (NB); 28) Greenwood \& Baker 1987 (ON); 29) Dechtiar \& Christie 1988 (ON); 30) Dechtiar et al. 1988 (ON); 31) Dechtiar \& Lawrie 1988 (ON); 32) Baggs \& McT. Cowan 1989 (NF); 33) Marcogliese \& Cone 1991b (NF); 34) Dubois et al. 1996 (QC)

Sterliadochona sp.
Site: intestine

Host: Acipenser fulvescens

Distribution: MB

Record: Choudhury \& Dick 1993

FAMILY TETRAMERIDAE

*Tetrameres sp.

Site: not specified

Host: Phoxinus eos

Distribution: QC

Record: Dubois et al. 1996

\section{SUPERFAMILY THELAZIODEA}

FAMILY RHABDOCHONIDAE

Rhabdochona canadensis Moravec and Arai, 1971

Site: intestine

Hosts: Catostomus commersoni $(10,11)$ Couesius plumbeus $(1,7)$

Esox lucius $(1,3,4)$

Hiodon alosoides $(10,11)$

Margariscus margarita (9)

Moxostoma anisurum $(10,11)$

Noturus flavus (1)

Percopsis omiscomaycus $(10,11)$

Phoxinus eos (9)

Phoxinus neogaeus (9)

Playtgobio gracilis (1)

Rhinichthys cataractae $(1,6,7,8)$

Salvelinus malma $(2,5)$

Stizostedion vitreum (1)

Distribution: $\quad$ AB, BC, LB, MB, ON, QC 
Records: 1) Moravec \& Arai 1971

(AB-Milk River); 2) Anonymous 1978 (BC); 3) Chinniah \& Threlfall 1978 (LB); 4) Thompson \& Threlfall 1978 (QC); 5) Arai \& Mudry 1983 (BC); 6) Dechtiar \& Christie 1988 (ON); 7) Dechtiar et al. 1988 (ON); 8) Dechtiar \& Lawrie 1988 (ON); 9) Dechtiar et al. 1989 (ON); 10) Szalai 1989 (MB); 11) Szalai et al. 1992 (MB)

Rhabdochona cascadilla Wigdor, 1918

Includes: Rhabdochona sp. of Bangham (1941) (partim) and of Bangham and Venard (1946) (partim)

Site: intestine

Hosts: Acipenser fulvescens $(7,8,9,18)$

Carpiodes cyprinus (1)

Catostomus catostomus $(4,6,11$,

14)

14)

Catostomus commersoni $(4,10,11$,

Catostomus macrocheilus (6)

Couesius plumbeus (6)

Culaea inconstans (17)

Etheostoma exile $(5,7,9)$

Hiodon tergisus (1)

Lota lota (7)

Margariscus margarita $(2,3,4)$

Moxostoma macrolepidotum (6)

Notropis hudsonius $(1,5,7,9)$

Oncorhynchus clarki (6)

Oncorhynchus mykiss $(6,16)$

Perca flavescens (10)

Percopsis omiscomaycus (10)

Phoxinius eos $(3,19)$

Prosopium williamsoni $(6,16)$

Ptychocheilus oregonensis $(6,13$, $15,16)$

Rhinichthys cataractae (6)
Richardsonius balteatus $(6,13,15)$

Salvelinus fontinalis (12)

Thymallus arcticus (6)

Distribution: $\quad$ AB, BC, MB, ON, QC

Records: 1$)$ Bangham \& Hunter 1939

(ON); 2) Bangham 1941 (ON); 3)

Bangham \& Venard 1946 (ON); 4)

Choquette 1951 (ON, QC); 5) Bangham 1951 (ON); 6) Bangham \& Adams 1954 (BC); 7) Bangham 1955 (ON); 8)

Dechtiar 1972b (ON); 9) Dechtiar 1972a (ON); 10) Collins \& Dechtiar 1974 (ON);

11) Leong 1975 (AB - Cold Lake); 12)

Mudry \& Anderson 1977 (AB - Babel

Creek); 13) Anonymous 1978 (BC); 14)

Leong \& Holmes 1981 (AB - Cold

Lake); 15) Arai \& Mudry 1983 (BC); 16)

Anonymous 1984 (BC); 17) Dechtiar et

al. 1988 (ON); 18) Choudhury \& Dick 1993 (MB); 19) Dubois et al. 1996 (QC)

Rhabdochona catostomi Kayton, Kritsky and Tobias, 1979

Site: intestine

Host: Catostomus catostomus

Distribution: $\quad$ AB

Record: Kayton et al. 1979 (AB - Nose Creek)

Rhabdochona cotti Gastafson, 1949

Site: intestine

Host: Cottus cognatus

Distribution: $\quad \mathrm{BC}, \mathrm{ON}$

Records: $\quad$ Bangham \& Adams 1954 (BC); Mudry \& Anderson 1977 (BC); Anonymous 1978 (BC); Arai \& Mudry 1983 (BC); Dechtiar et al. 1989 (ON) 
Rhabdochona decaturensis Gustafson, 1949

Site: intestine

Hosts: Notropis hudsonius $(1,2,3)$

Noturus flavus (1)

Distribution: ON

Records: 1) Dechtiar \& Christie 1988; 2)

Dechtiar et al. 1988; 3) Dechtiar \&

Lawrie 1988

Rhabdochona kisutchi Margolis, Moravec and MacDonald, 1975

Site: intestine

Hosts: Oncorhynchus kisutch (1)

Oncorhynchus mykiss $(2,3)$

Prosopium williamsoni $(2,3)$

Salvelinus malma $(2,3,4)$

Distribution: $\quad \mathrm{BC}$

Records: 1) Margolis et al. 1975; 2)

Anonymous 1978; 3) Arai \& Mudry 1983;

4) Anonymous 1984

Rhabdochona milleri Choquette, 1951

Site: intestine

Hosts: Carpiodes cyprinus (8)

Moxostoma macrolepidotum (1, 2,

$3,7)$

Oncorhynchus clarki (4)

Oncorhynchus mykiss (4)

Prosopium coulteri (4)

Prosopium williamsoni $(5,6)$

Rhinichthys cataractae $(5,6)$

Salvelinus fontinalis (4)

Distribution: $\quad$ AB $, \mathrm{BC}, \mathrm{ON}, \mathrm{QC}$

Records: 1) Choquette 1951 (QC); 2)

Moravec \& Arai 1971 (ON); 3) Dechtiar 1972b (ON); 4) Mudry \& Anderson 1977 (AB - Snowflake Lake (S. fontinalis), Crypt Lake (O. clarki), BC (O. mykiss, $P$. coulteri, S. fontinalis, $S$. malma); 5) Anonymous 1978 (BC); 6) Arai \& Mudry 1983 (BC); 7) Dechtiar \& Christie 1988 (ON); 8) Dechtiar \& Nepszy 1988 (ON)

Rhabdochona ovifilamenta Weller, 1938

Synonym: Rhabdochona laurentiana Lyster, 1940

Includes: Rhabdochona fortunowi of Kussat (1969); Rhabdochona sp. of Arai and Kussat (1967)

Site: intestine

Hosts: Catostomus catostomus $(3,4,7)$ Catostomus commersoni $(1,8,9$, 11, 12)

Catostomus platyrhychus $(3,4,7$,

8)

Perca flavescens $(5,6,10)$

Salvelinus fontinalis (2)

Distribution: AB, BC, QC

Records: 1) Lyster 1940 (QC); 2)

Choquette 1948a (QC); 3) Kussat 1966

(AB - Bow River); 4) Arai \& Kussat

1967 (AB - Bow River); 5) Tedla \&

Fernando 1969a (ON); 6) Tedla 1969

(ON); 7) Kussat 1969 (AB - Bow

River); 8) Moravec \& Arai 1971 (AB -

Highwood River); 9) Dechtiar \& Christie 1988 (ON); 10) Dechtiar \& Lawrie 1988

(ON); 11) Dechtiar \& Nepszy 1988 (ON);

12) Dechtiar et al. 1989 (ON)

Rhabdochona zacconis Yamaguti, 1935

Site: intestine

Host: Catostomus macrocheilus

Distribution: $\quad \mathrm{BC}$ 
Records: Anonymous 1978; Arai \& Mudry 1983

Rhabdochona sp.

Site: intestine

Hosts: Catostomus catostomus $(7,8,11$, 15)

Catostomus commersoni $(1,2,8)$

Catostomus macrocheilus $(11,15)$

Catostomus platyrhynchus (7)

Coregonus clupeaformis (2)

Cottus cognatus $(11,15)$

Couesius plumbeus $(4,6)$

Etheostoma exile (17)

Notropis atherinoides (19)

Notropis hudsonius (3)

Oncorhynchus clarki (10)

Oncorhynchus kisutch $(5,14,16)$

Oncorhynchus mykiss $(11,13,15$,

16)

Perca flavescens $(1,4,6,9,12)$

Percopsis omiscomaycus (18)

Pimephales promelas (2)

Prosopium williamsoni $(11,15,16$, 20)

Ptychocheilus oregonensis $(11,15)$

Richardsonius balteatus $(11,15)$

Salvelinus fontinalis $(5,10)$

Salvelinus fontinalis X Salvelinus

namaycush (12)

Salvelinus malma $(11,14,15,16)$

Distribution: $\quad$ AB, BC, LB, ON

Records: 1) Bangham 1941 (ON); 2)

Bangham \& Venard 1946 (ON); 3)

Stewart-Hay 1951g (MB); 4) Bangham

1951 (ON); 5) Bangham \& Adams 1954

(BC); 6) Bangham 1955 (ON); 7) Kussat

1969 (AB - Bow River); 8) Threlfall \&

Hanek 1970a (LB); 9) Tedla \& Fernando

1972 (ON); 10) Mudry \& Anderson
1977 (AB - Utopia Lake (O. clarki),

Vista Lake (S. fontinalis); 11)

Anonymous 1978 (BC); 12) Dechtiar \&

Berst 1978 (ON); 13) Pybus \& Samuel

1978 (AB - Hardisty Creek); 14)

Anonymous 1981 (BC); 15) Arai \&

Mudry 1983 (BC); 16) Anonymous 1984

(BC); 17) Dechtiar et al. 1988 (ON); 18)

Dechtiar \& Lawrie 1988 (ON); 19) Byrne

1992 (ON); 20) Nener et al. 1995 (BC)

Rhabdochonidae gen. sp.

Site: intestine

Host: Acipenser fulvescens

Distribution: MB, SK

Record: Choudhury et al. 1990

\section{SUPERFAMILY ACUARIOIDEA}

\section{FAMILY ACUARIIDAE}

*Cosmocephalus obvelatus (Creplin, 1825)

Seurat, 1919

Site: mesenteries

Hosts: Gasterosteus aculeatus $(1,2)$

Notropis hudsonius (1)

Oncorhynchus mykiss (1)

Pungitius pungitius $(2,3)$

Distribution: EXP, NS, ON

Records: $\quad$ 1) Wong \& Anderson 1982

(EXP, ON); 2) Marcogliese 1992 (NS); 3)

Marcogliese 1995 (NS)

*Paracuaria adunca (Creplin, 1846)

Anderson and Wong, 1981 
Site: mesenteries

Hosts: Gasterosteus aculeatus $(1,2)$

Notropis hudsonius (1)

Pungitius pungitius $(2,3)$

Distribution: NS, ON

Records: 1) Anderson \& Wong 1982

(ON); 2) Marcogliese 1992 (NS); 3)

Marcogliese 1995 (NS)

*Spiruridae gen. sp.

Sites: coelom, intestinal wall, mesenteries, musculature, ovaries, pyloric caeca, stomach wall, swim bladder

Hosts: Oncorhynchus mykiss (2) Salvelinus fontinalis (1)

Distribution: $\quad$ BC, QC

Records: 1) Choquette 1948a (QC); 2) Anonymous 1984 (BC)

*Spirurinae gen. sp.

Site: mesenteries

Host: Myoxocephalus thompsoni

Distribution: ON

Record: Black \& Lankester 1981a

**Nematoda gen. sp.

Includes: Agamonema sp. auct.; Agamospirura sp. auct.

Sites: mesenteries, musculature, viscera

Hosts: Acipenser fulvescens (29)

Catostomus catostomus (30)

Catostomus commersoni $(6,15$,

30)

Coregonus artedii $(18,22,23)$

Coregonus clupeaformis $(2,3,11$,

$12,20,22,23)$
Cottus cognatus (35)

Couesius plumbeus $(24,25)$

Culaea inconstans $(6,31,42,43)$

Esox lucius $(12,24,40)$

Etheostoma exile (6)

Hiodon alosoides (30)

Lota lota $(12,17,22,28)$

Moxostoma anisurum (10)

Notropis hudsonius (6)

Oncorhynchus clarki (7)

Oncorhynchus kisutch (36)

Oncorhynchus mykiss $(1,34,36$,

39)

Perca flavescens $(6,7,8,18,19$, 40, 41)

Percina caprodes (6)

Percopsis omiscomaycus $(6,21,26)$

Phoxinus eos $(8,14)$

Phoxinus neogaeus $(8,14)$

Prosopium cylindraceum (32)

Prosopium williamsoni $(34,36,39)$

Salvelinus confluentus (1)

$32,33)$

Salvelinus fontinalis $(4,5,9,15,16$,

Salvelinus malma $(36,39)$

Salvelinus namaycush $(9,22,27$,

$32,37,38)$

Stizostedion vitreum $(6,9,10,18)$

Thymallus arcticus (13)

Distribution: $\quad \mathbf{A B}, \mathrm{BC}, \mathrm{LB}, \mathrm{MB}, \mathrm{NS}, \mathrm{NT}$, ON, QC, SK

Records: 1) Neave \& Bajkov 1929

[AB- Creek at Oldfort Point, Buffalo

Praire Lake, Mingo Creek (O. mykiss),

Jacques Lake (S. confluentus); 2)

Bajkov 1930 (MB); 3) Hart 1931 (ON);

4) Ricker 1932 (ON); 5) Richardson 1935 (ON); 6) Bangham \& Hunter 1939 (ON);

7) Rawson 1939 (AB - Moraine Lake);

8) Bangham 1941 (ON); 9) MacLulich

1943b (ON); 10) McLeod 1943 (MB);

11) Miller 1944b (NT); 12) Miller 1945d 
(AB - Lake Athabasca); 13) Miller 1946a (NT); 14) Bangham \& Venard 1946 (ON); 15) Fantham \& Porter 1948 (QC); 16) Choquette 1948a (QC); 17) Rawson 1951 (NT); 18) Stewart-Hay 1951f (MB); 19) Stewart-Hay $1951 \mathrm{~g}$ (MB); 20) Wheaton \& Hazen 1951 (SK); 21) Bangham 1951 (ON); 22) StewartHay 1953a (MB); 23) Stewart-Hay 1953b (MB); 24) Stewart-Hay 1953c (MB); 25) Bangham \& Adams 1954 (BC); 26) Bangham 1955 (ON); 27) Rawson 1961 (SK); 28) Fillion 1961 (AB - Cold Lake, Lac la Nonne, Lac Ste. Anne); 29) Harkness \& Dymond 1961 (ON); 30) Reed 1962 (SK); 31) Dechtiar 1972a (ON); 32) Hicks \& Threlfall 1973 (LB); 33) Frantsi et al. 1975 (NS); 34) Mudry \& Anderson 1977 [AB - Edith Lake, Moraine Lake (O. mykiss), Lower Waterton Lake ( $P$. williamsoni)]; 35) Pybus \& Samuel 1978 (AB - near Hinton); 36) Anonymous 1981 (BC); 37) Stewart \& Bernier 1984 (BC); 38) McAllister \& Mudry 1983 (AB - Cold Lake); 39) Anonymous 1984 (BC); 40) Samuel 1985 (AB - Jarvis Lake); 41) Dechtiar \& Christie 1988 (ON); 42) Dechtiar et al. 1988 (ON); 43) Dechtiar et al. 1989 (ON)

PHYLUM ACANTHOCEPHALA

ORDER EOACANTHOCEPHALA

FAMILY NEOECHINORHYNCHIDAE

Neoechinorhynchus carpiodi Dechtiar, 1968
Site: intestine

Host: Carpiodes cyprinus

Distribution: MB, ON

Records: Dechtiar 1968 (ON); Dechtiar 1972b (ON); Szalai \& Dick 1987a (MB); Szalai \& Dick 1987b (MB); Dechtiar \& Nepszy 1988 (ON); Szalai et al. 1988 (MB); Szalai et al. 1992 (MB)

Neoechinorhynchus crassus Van Cleave, 1919

Sites: intestine, pyloric caeca

Hosts: Carpiodes cyprinus (6)

Catostomus catostomus $(5,8,10$,

$12,13,15)$

Catostomus commersoni $(1,2,3$,

$5,7,10,11,12,14,15,16)$

Catostomus macrocheilus (4)

Coregonus clupeaformis (9)

Moxostoma anisurum (16)

Moxostoma macrolepidotum (11)

Perca flavescens (16)

Distribution: BC, LB, MB, ON, SK

Records: 1) Bangham \& Hunter 1939

(ON); 2) Van Cleave 1949 (SK); 3)

Stewart-Hay 1953c (MB); 4) Bangham \& Adams 1954 (BC); 5) Bangham 1955

(ON); 6) Dechtiar 1972b (ON); 7)

Dechtiar 1972a (ON); 8) Chinniah \& Threlfall 1978 (LB); 9) Pybus \& Samuel 1978 (AB - Blue Lake, Jarvis Lake); 10) Courtney 1987 (ON); 11) Dechtiar \& Christie 1988 (ON); 12) Dechtiar et al. 1988 (ON); 13) Dechtiar \& Lawrie 1988 (ON); 14) Dechtiar \& Nepszy 1988 (ON); 15) Dechtiar et al. 1989 (ON); 16) Szalai et al. 1992 (MB)

Neoechinorhynchus cylindratus (Van 
Cleave, 1913) Van Cleave, 1919

Synonyms: Neorhynchus cylindratus Van Cleave, 1913; Eorhynchus cylindratus (Van Cleave, 1913) Van Cleave, 1914

Site: intestine

Hosts: Catostomus commersoni (2) Coregonus clupeaformis $(8,10)$ Esox lucius $(3,11)$

Lota lota $(3,13)$

Perca flavescens $(3,4,5,6,7)$

Richardsonius balteatus $(9,10)$

Salvelinus fontinalis (2)

Stizostedion canadense (3)

13)

Stizostedion vitreum $(1,3,4,12$,

Distribution: $\quad \mathrm{BC}, \mathrm{MB}, \mathrm{ON}, \mathrm{QC}$

Records: 1) Bangham \& Hunter 1939

(ON); 2) Lyster 1940a (QC); 3) Bangham

1955 (ON); 4) Dickson 1964 (MB); 5)

Tedla \& Fernando 1969a (ON); 6) Tedla

1969 (ON); 7) Tedla \& Fernando 1972

(ON); 8) Watson 1977 (MB); 9)

Anonymous 1978 (BC); 10) Arai \&

Mudry 1983 (BC); 11) Anthony 1983

(ON); 12) Dechtiar \& Nepszy 1988 (ON);

13) Anthony 1987 (ON)

Neoechinorhynchus distractus Van Cleave, 1949

Site: intestine

Hosts: Catostomus commersoni

Moxostoma anisurum

Distribution: MB

Record: $\quad$ Szalai et al. 1992

Neoechinorhynchus notemigoni Dechtiar, 1967
Site: intestine

Host: Notropis hudsonius

Distribution: ON

Record: Dechtiar \& Lawrie 1988

Neoechinorhynchus pungitius Dechtiar, 1971

Site: intestine

Hosts: Culaea inconstans $(1,2,3)$

Perca flavescens $(1,2)$

Pungitius pungitius $(1,2)$

Distribution: ON

Records: 1) Dechtiar 1971; 2) Dechtiar

et al. 1988; 3) Dechtiar et al. 1989

Neoechinorhynchus rutili (O. F. Miller, 1780) Hamann, 1892

Site: intestine

Hosts: Acipenser fulvescens (19)

Catostomus catostomus (3)

Catostomus commersoni (12)

Catostomus macrocheilus (3)

Coregonus artedii (1)

Coregonus clupeaformis $(13,15)$

Cottus cognatus (11)

Cottus ricei (20)

Couesius plumbeus $(3,20)$

Culaea inconstans $(4,6,19,20,23$,

24)

Esox lucius $(4,11)$

Gasterosteus aculeatus $(2,3,5,9)$

Lota lota $(3,4,11,13,15)$

Notropis hudsonius $(4,7,19,20$,

21)

Oncorhynchus clarki $(2,3)$

Oncorhynchus kisutch (3)

Oncorhynchus mykiss $(3,4,13,15$, 
Perca flavescens $(7,818,20,22)$

Phoxinus neogaeus (20)

Prosopium coulteri (15)

Prosopium williamsoni $(3,13,15$, $16,17)$

Ptychocheilus oregonensis (2, 3,

$13,16,17)$

20)

Pungitius pungitius $(2,4,10,14$, $16,17)$

Salvelinus fontinalis $(2,3,21)$

Salvelinus malma $(3,13,15)$

Salvelinus namaycush $(11,13,15$,

$16,17)$

Stizostedion vitreum (19)

Thymallus arcticus $(11,13,15)$

Distribution: $\quad$ AB, BC, LB, MB, NF, NT, ON, SK, YT

Records: 1) Van Cleave \& Bangham 1949 (SK); 2) Van Cleave \& Lynch 1950 (BC); 3) Bangham \& Adams 1954 (BC); 4) Bangham 1955 (ON); 5) Hanek \& Threlfall 1970a (LB); 6) Dechtiar 1972a (ON); 7) Dechtiar 1972b (ON); 8) Collins \& Dechtiar 1974 (ON); 9) Lester 1975 (BC); 10) Dickinson \& Threlfall 1976 (NF); 11) Arthur et al. 1976 (YT); 12) Mudry \& Anderson 1977 (AB - Lower Waterton Lake); 13) Anonymous 1978 (BC); 14) Curtis 1982 (NT); 15) Arai \& Mudry 1983 (BC); 16) Anonymous 1983 (BC); 17) Anonymous 1984 (BC); 18) Poole \& Dick 1985 (MB); 19) Dechtiar \& Christie 1988 (ON); 20) Dechtiar et al. 1988 (ON); 21) Dechtiar \& Lawrie 1988 (ON); 22) Dechtiar \& Nepszy 1988 (ON); 23) Dechtiar et al. 1989 (ON); 24)

McLennon \& Shires 1995 (ON)

Neoechinorhynchus saginatus Van Cleave and Bangham, 1949

Site: intestine

Hosts: Catostomus catostomus (1)

Catostomus commersoni (2)

Catostomus macrocheilus (1)

Lota lota (1)

Oncorhynchus kisutch (1)

Oncorhynchus mykiss (1)

Prosopium williamsoni (1)

Ptychocheilus oregonensis (1)

Distribution: $\mathrm{BC}, \mathrm{ON}$

Records: 1) Anonymous 1981 (BC); 2)

Mackie et al. 1983 (ON)

Neoechinorhynchus salmonis Ching, 1984

Includes: Neoechinorhynchus saginatus Van Cleave and Bangham, 1949 of Anonymous (1981)

Sites: intestine, pyloric caeca

Hosts: Catostomus catostomus $(1,2,3,4)$ Catostomus macrocheilus $(1,3,4)$ Coregonus clupeaformis $(2,3)$

Lota lota $(1,3,4)$

Oncorhynchus kisutch $(1,3)$

Oncorhynchus mykiss $(1,2,3,4)$

Prosopium williamsoni (4)

Ptychocheilus oregonensis (1, 2, 3,

4)

Savelinus malma (3)

Salvelinus namaycush $(2,3)$

Distribution: $\quad \mathrm{BC}$

Records: 1) Anonymous $1981 ; 2$ )

Anonymous 1983; 3) Anonymous 1984;

4) Ching 1984b

Neoechinorhynchus strigosus Van Cleave, 1949 
Site: intestine

Hosts: Catostomus catostomus $(1,2)$

Catostomus commersoni $(1,2,3,4$, $5,7,8)$

Esox lucius $(4,5)$

Oncorhynchus kisutch $(4,5)$

Stizostedion vitreum (6)

Distribution: AB, LB, MB, ON

Records: 1) Bangham 1955 (ON); 2)

Threlfall \& Hanek 1970a (LB); 3)

Dechtiar 1972a (ON); 4) Leong 1975

(AB - Cold Lake); 5) Leong \& Holmes

1981 (AB - Cold Lake); 6) Poole \& Dick

1985 (MB); 7) Dechtiar et al. 1988 (ON);

8) Dechtiar et al.1989

Neoechinorhynchus tenellus (Van Cleave, 1913)

Synonym: Neorhynchus tenellus Van

Cleave, 1913

Site: intestine

Hosts: Acipenser fulvescens (10)

Esox lucius $(1,3,5,6,7,8)$

Perca flavescens (4)

Stizostedion canadense (3)

$8,9)$

Stizostedion vitreum $(2,3,4,5,7$,

Distribution: $\mathrm{MB}, \mathrm{ON}$

Records: 1) Bangham \& Hunter 1939

(ON); 2) Dechtiar 1972a (ON); 3)

Dechtiar 1972b (ON); 4) Anthony 1978b

(ON); 5) Dechtiar \& Christie 1988 (ON);

6) Dechtiar et al. 1988 (ON); 7) Dechtiar

\& Lawrie 1988 (ON); 8) Dechtiar \&

Nepszy 1988 (ON); 9) Szalai et al. 1992

(MB); 10) Choudhury \& Dick 1993 (MB)

Neoechinorhynchus tumidus Van Cleave and Bangham, 1949
Site: intestine

Hosts: Coregonus artedii $(1,4,12)$

Coregonus clupeaformis $(1,3,4,5$,

$6,8,9,10,11,12,13)$

Cottus cognatus (6)

Prosopium cylindraceum $(2,6,11)$

Prosopium williamsoni (6)

Salvelinus fontinalis $\mathrm{X}$ Salvelinus

namaycush $(7,11)$

Thymallus arcticus (6)

Distribution: $\quad$ AB, ON, SK, YT

Records: 1) Van Cleave \& Bangham

1949 (ON); 2) Bangham 1955 (ON); 3)

Dechtiar 1972a (ON); 4) Dechtiar 1972b

(ON); 5) Collins \& Dechtiar 1974 (ON);

6) Arthur et al. 1976 (YT); 7) Dechtiar \&

Berst 1978 (ON); 8) Samuel 1981 (AB -

Blue Lake, Jarvis Lake); 9) Samuel

1985 (AB - Blue Lake, Jarvis Lake);

10) Dechtiar \& Christie 1988 (ON); 11)

Dechtiar et al. 1988 (ON); 12) Dechtiar \&

Lawrie 1988 (ON); 13) Stock 1988 (AB -

Jarvis Lake)

Neoechinorhynchus venustus Lynch, 1936

Synonym: Neoechinorhynchus cristatus Lynch, 1936

Site: intestine

Hosts: Catostomus catostomus $(1,2,3,4$,

$5,11,12,13,14,15,17)$

Catostomus commersoni $(2,3,4,7$,

$8,12,14,16,17,18,19,20,21)$

Catostomus macrocheilus (1, 12 ,

$13,14,15)$

Moxostoma anisurum (21)

Perca flavescens (9)

Ptychocheilus oregonensis $(13,15)$

Pungitius pungitius $(6,10)$

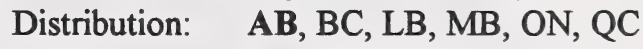

Records: 1) Bangham \& Adams 1954 
(BC); 2) Kussat 1966 (AB - Big Hill Creek, Bow River); 3) Arai \& Kussat 1967 (AB - Big Hill Creek, Bow River); 4) Kussat 1969 [AB - Big Hill Creek, Bow River ( $C$. commersoni), Bow River (C. catostomus)]; 5) Threlfall \& Hanek 1970a (LB); 6) Hanek \& Threlfall 1970b (LB); 7) Dechtiar 1972a (ON); 8) Dechtiar 1972b (ON); 9) Tedla \& Fernando 1972 (ON); 10) Hanek \& Molnar 1974 (QC); 11) Gordon et al. 1978 (QC); 12) Anonymous 1978 (BC); 13) Anonymous 1981 (BC); 14) Arai \& Mudry 1983 (BC); 15) Anonymous 1984 (BC); 16) Dechtiar \& Christie 1988 (ON); 17) Dechtiar et al.1988 (ON); 18) Dechtiar \& Lawrie 1988 (ON); 19) Dechtiar \& Nepszy 1988 (ON); 20) Dechtiar et al. 1989 (ON); 21) Szalai et al. 1992 (MB);

Remarks: Neoechinorhynchus vemustus and $N$. cristatus were placed in synonymy by Petrochenko (1956). The synonymy should have resulted in the retention of $N$. venustus with $N$. cristatus as the junior synonym since the description of $N$. venustus appeared "in print" before that of the latter.

Neoechinorhynchus sp.

Site: intestine

Hosts: Carpiodes cyprinus (6) Catostomus catostomus (5) Catostomus commersoni $(4,6,7,8$ $13,14)$

Coregonus artedii (1)

Coregonus clupeaformis $(14,18)$

Culaea inconstans (12)

Esox lucius (2)

Gasterosteus aculeatus (16)
Notropis atherinoides (10)

Notropis hudsonius (10)

Oncorhynchus mykiss (17)

Perca flavescens (11)

Percopsis omiscomaycus (9)

Pimephales promelas (21)

Prosopium williamsoni (17)

Richardsonius balteatus (17)

Salvelinus fontinalis $(19,20)$

Salvelinus namaycush (17)

Stizostedion canadense (3)

Stizostedion vitreum (8)

Distribution: $\quad \mathbf{A B}, \mathrm{BC}, \mathrm{MB}, \mathrm{NB}, \mathrm{NT}$, ON

Records: 1) Pritchard 1931 (ON); 2) Cushing \& Bacal 1932 (ON); 3) Bangham \& Hunter 1939 (ON); 4) Bangham 1941 (ON); 5) Rawson 1951 (NT); 6) StewartHay 1951b (MB); 7) Stewart-Hay 1952a (MB); 8) Stewart-Hay 1952b (MB); 9) Bangham 1955 (ON); 10) Dechtiar 1972a (ON); 11) Dechtiar 1972b (ON); 12) Kakonge 1972 (ON); 13) Mudry \& Arai 1973b (AB - Nose Creek); 14) Pybus \& Samuel 1978 (AB - Blue Lake, Jarvis Lake); 15) Chan 1980 (ON); 16) Curtis 1982 (NT); 17) Anonymous 1984 (BC);

18) Samuel 1985 (AB - Jarvis Lake);

19) Frimeth 1987a (NB); 20) Frimeth $1987 b$ (NB); 21) Dechtiar \& Christie $1988(\mathrm{ON})$

Octospinifer macilentus Van Cleave, 1918

Includes: Octospinifer sp. of Mudry and Arai (1973b)

Site: intestine

Hosts: Catostomus catostomus $(2,6,13$, 15)

Catostomus commersoni $(1,2,3,4$, $5,6,7,8,9,10,11,12,13,14,15,16$, 
$17,18,19)$

Catostomus macrocheilus $(13,15)$

Distribution: AB, BC, ON, QC

Records: 1) Bangham \& Hunter 1939

(ON); 2) Bangham 1941 (ON); 3)

Bangham \& Venard 1946 (ON); 4) Van

Cleave 1949 (ON); 5) Bangham 1955

(ON); 6) Kussat 1966 (AB - Big Hill

Creek, Bow River); 7) Arai \& Kussat

1967 (AB - Big Hill Creek, Bow River);

8) Kussat 1969 (AB - Big Hill Creek,

Bow River); 9) Dechtiar 1972a (ON);

10) Beerman 1972 (AB - Big Hill

Creek, Bow River); 11) Mudry \& Arai 1973b (AB - Nose Creek); 12) Beerman et al. 1974 (AB - Big Hill Creek, Bow

River); 13) Anonymous 1978 (BC); 14)

Chan 1980 (ON); 15) Arai \& Mudry 1983

(BC); 16) Dechtiar \& Christie 1988 (ON);

17) Dechtiar et al. 1988 (ON); 18)

Dechtiar \& Nepszy 1988 (ON); 19)

Dubois et al. 1996 (QC)

\section{FAMILY TENUISENTIDAE}

Tanaorhamphus sp.

Includes: Tanorhynchus sp. of StewartHay (1952b)

Site: intestine

Host: Catostomus commersoni

Distribution: $\quad \mathrm{MB}$

Record: Stewart-Hay $1952 b$

ORDER PALAEACANTHOCEPHALA

SUPERFAMILY

ECHINORHYNCHOIDEA

\section{FAMILY ECHINORHYNCHIIDAE}

Acanthocephalus dirus (Van Cleave, 1931)

Van Cleave and Townsend, 1936

Synonym: Acanthocephalus jacksoni Bullock, 1962

Site: intestine

Hosts: Catostomus catostomus (5)

Catostomus commersoni $(1,6)$

Coregonus clupeaformis $(1,3)$

Cottus cognatus $(1,3)$

Cottus ricei (4)

Culaea inconstans (4)

Esox lucius $(5,6)$

Lota lota $(4,7)$

Oncorhynchus kisutch (3)

Oncorhynchus mykiss (5)

Perca flavescens $(1,3,5)$

Percopsis omiscomaycus (5)

Prosopium cylindraceum (7)

Pungitius pungitius (4)

Salmo trutta (4)

Salvelinus fontinalis $(3,7)$

Salvelinus fontinalis $\mathrm{X}$ Salvelinus namaycush $(1,2,4)$

Salvelinus namaycush (7)

Distribution: $\mathrm{ON}$

Records: 1) Collins \& Dechtiar 1974; 2)

Dechtiar \& Berst 1978; 3) Dechtiar \&

Christie 1988; 4) Dechtiar et al. 1988; 5)

Dechtiar \& Lawrie 1988; 6) Dechtiar \&

Nepszy 1988; 7) Dechtiar et al. 1989

Acanthocephalus sp.

Site: intestine

Hosts: Coregonus clupeaformis

Esox lucius

Stizostedion vitreum 
Distribution: $\quad$ MB

Record: $\quad$ McTavish 1952

Echinorhynchus gadi Zoega in O. F. Muller, 1776

Synonym: Echinorhynchus vancleavei Golvan, 1969

Site: intestine

Hosts: Coregonus clupeaformis (3) Salvelinus fontinalis (1) Salvelinus namaycush $(2,3)$

Distribution: NT, QC

Records: 1) Black 1981 (QC); 2)

Stewart \& Bernier 1982 (NT); 3) Shostak et al. 1986 (NT)

Echinorhynchus lateralis Leidy, 1851

Synonyms: Acanthocephalus lateralis

(Leidy, 1851); Metechinorhynchus

lateralis (Leidy, 1851) Golvan, 1969

Site: intestine

Hosts: Acipenser fulvescens (21)

Catostomus catostomus (3)

Coregonus artedii (21)

Coregonus clupeaformis $(4,5,10$,

11)

Esox lucius $(6,15)$

Gasterosteus aculeatus $(7,17)$

Oncorhynchus kisutch (21)

Oncorhynchus mykiss $(4,21)$

Perca flavescens (3)

Prosopium cylindraceum $(10,21)$

Pungitius pungitius (8)

Salmo trutta $(4,5,9)$

Salvelinus fontinalis $(1,2,3,4,5$,

$9,11,12,13,14,15,16,17,18,19,20$,

$22,23,24)$

Salvelinus namaycush $(10,21)$
Distribution: LB, NB, NF, NS, ON, QC Records: 1) Richardson 1936 (QC); 2) Choquette 1948a (QC); 3) Fantham \& Porter 1948 (QC); 4) Sandeman \& Pippy 1967 (NF); 5) Threlfall \& Hanek 1970c (NF); 6) Threlfall \& Hanek 1970b (LB); 7) Hanek \& Threlfall 1970a (LB, NF); 8) Hanek \& Threlfall 1970b (NF); 9) Hanek \& Threlfall 1970d (NF); 10) Dechtiar 1972a (ON); 11) Hicks \& Threlfall 1973 (LB); 12) Hare \& Frantsi 1974 (NB); 13) Frantsi et al. 1975 (NS); 14) Thompson \& Threlfall 1978 (QC); 15) Chinniah \& Threlfall 1978 (LB); 16) Black 1981a (QC); 17) Cone \& Ryan 1984 (NF); 18) Frimeth 1987a (NB); 19) Frimeth 1987b (NB); 20) Dechtiar et al. 1988 (ON); 21) Dechtiar \& Lawrie 1988 (ON); 22) Baggs \& McT. Cowan 1989 (NF); 23)

Marcogliese \& Cone 1991b (NF); 24)

Dubois et al. 1996 (QC)

Echinorhynchus leidyi Van Cleave, 1924

Synonyms: Echinorhynchus salvelini Linkins in Ward and Whipple, 1918; Metechinorhynchus leidyi (Van Cleave, 1924) Golvan, 1969

Site: intestine

Hosts: Acipenser fulvescens $(15,16)$

Catostomus catostomus (8)

Catostomus commersoni (8)

Coregonus artedii $(10,12)$

Coregonus clupeaformis $(10,11$,

12)

Esox lucius $(10,12,13)$

Lota lota $(4,8,12)$

Oncorhynchus mykiss $(8,11)$

Salvelinus malma (1)

Salvelinus namaycush $(1,2,3,5,6$,

$7,8,9,11,12,14)$ 
Distribution: $\quad$ AB, EXP, MB, NT, ON, QC, SK

Records: 1) Van Cleave 1920 (ON); 2) Cooper 1921 (ON); 3) Richardson 1942 (QC); 4) Miller 1944 b (NT); 5) Miller 1945e (AB - Lake Athabasca); 6) Miller 1946b (NT); 7) Miller \& Kennedy 1948 (NT); 8) Bangham 1955 (ON); 9) Rawson 1959 (SK); 10) Shostak \& Dick 1982 (MB); 11) Prychitko \& Nero 1983 (EXP, ON); 12) Shostak et al. 1986 (MB, NT); 13) Shostak \& Dick 1986 (MB); 14) Dechtiar et al. $1988(\mathrm{ON}) ; 15)$ Choudhury et al. 1990 (MB); 16) Choudhury \& Dick 1993 (MB)

Echinorhynchus salmonis O. F. Müller, 1784

Synonyms: Metechinorhynchus salmonis (O. F. Müller, 1784) Petrochenko, 1956; Echinorhynchus coregoni Linkins in Van Cleave, 1919

Site: intestine

Hosts: Acipenser fulvescens $(10,34,35$, 36)

Catostomus catostomus $(7,10,20$, $22,28,33,35$ )

Catostomus commersoni $(10,17$, $20,21,23,28,33$ )

Coregonus artedii $(6,10,15,18$, $19,20,21,22,23,24,26,28,29,32,34$, 35,36 )

Coregonus clupeaformis $(3,7,8,9$, $10,15,16,17,18,19,20,21,22,23,26$, $28,29,30,34,35,36,39$ )

Couesius plumbeus $(10,35)$

Esox lucius $(10,15,16,19,20,21$, $22,25,27,28,29,34,35,37)$

Gasterosteus aculeatus $(34,38)$

Lota lota $(3,9,10,11,15,19,20$,
$21,28,35,36)$

Myoxocephalus thompsoni (10)

Notropis hudsonius (10)

Oncorhynchus kisutch $(19,20,21$,

$24,28,34,35,36$ )

Oncorhynchus mykiss $(10,34,35)$

Perca flavescens $(10,12,13,14$,

17, 22, 24, 25, 27)

Percopsis omiscomaycus $(10,17$, 34, 36)

Prosopium cylindraceum $(10,35$, 36)

28)

Pungitius pungitius $(18,19,20,21$,

Salmo trutta (35)

Salvelinus fontinalis $(33,34,38)$

Salvelinus fontinalis $\mathrm{X}$ Salvelinus

namaycush $(17,23,35)$

Salvelinus malma (1)

Salvelinus namaycush $(1,2,5,7$,

$10,15,18,19,20,21,25,28,31,32,34$, $35,36)$

Stizostedion canadense $(10,15)$

Stizostedion vitreum $(15,16,20,21$, $28,34,36$ )

Thymallus arcticus (4)

Distribution: $\quad$ AB, BC, CA, MB, NT, ON

Records: 1) Van Cleave 1920 (NT); 2)

Cooper 1921 (NT); 3) Bangham \& Hunter 1939 (ON); 4) Miller 1946b (NT);

5) Miller \& Kennedy 1948 (NT); 6)

Rawson 1951 (NT); 7) Stewart-Hay 1953a (MB); 8) Stewart-Hay 1953b (MB); 9) Bangham \& Adams 1954 (BC); 10) Bangham 1955 (ON); 11) Fillion 1961 (AB - Cold Lake); 12) Tedla \& Fernando 1969d (ON); 13) Tedla \& Fernando 1970b (ON); 14) Tedla \& Fernando 1972 (ON); 15) Dechtiar 1972a (ON); 16) Dechtiar 1972b (ON); 17) Collins \& Dechtiar 1974 (ON); 18) Leong \& Holmes 1974a (AB - Cold Lake); 19) 
Leong \& Holmes 1974b (AB - Cold

Lake); 20) Leong 1975 (AB - Cold

Lake); 21) Holmes et al. 1977 (AB -

Cold Lake); 22) Watson 1977 (MB); 23)

Dechtiar \& Berst 1978 (ON); 24) Lester

\& Wright 1978 (ON); 25) Pybus \&

Samuel 1978 (AB - Angling Lake, Cold

Lake); 26) Watson \& Dick 1979 (MB);

27) Watson \& Dick 1980 (MB); 28)

Leong \& Holmes 1981 (AB - Cold

Lake); 29) Shostak \& Dick 1982 (MB);

30) McCart et al. 1982 (AB -

Athabasca River, Lake Athabasca); 31)

Stewart \& Bernier 1983 (NT); 32)

Shostak et al. 1986 (MB, NT); 33)

Courtney 1987 (ON); 34) Dechtiar \&

Christie 1988 (ON); 35) Dechtiar et al.

1988 (ON); 36) Dechtiar \& Lawrie 1988

(ON); 37) Dechtiar \& Nepszy 1988 (ON);

38) Curtis 1988 (CA); 39) Hobbs

(undated) (AB - Lesser Slave Lake)

Echinorhynchus sp.

Includes: Metechinorhynchus sp.

Sites: intestine, mesenteries, stomach

Hosts: Coregonus artedii (3)

Coregonus clupeaformis $(1,2,6)$

Etheostoma exile (2)

Lota lota (8)

Percina caprodes (2)

Salvelinus fontinalis (5)

Salvelinus namaycush (6)

Stizostedion vitreum (2)

Thymallus arcticus (4)

Distribution: LB, NT, ON

Records: 1) Hart 1931 (ON); 2)

Bangham \& Hunter 1939 (ON); 3) Miller

1945e (NT); 4) Miller 1946a (NT); 5)

Munroe 1949 (LB); 6) Stewart \& Bernier

1983 (NT); 7) Stewart \& Bernier 1984
(NT); 8) Anthony 1987 (ON)

\section{FAMILY LEPTORHYNCHOIDIDAE}

**Leptorhynchoides thecatus (Linton, 1891) Kostylev, 1924

Synonym: Echinorhynchus thecatus Linton, 1891

Sites: intestine, mesenteries

Hosts: Acipenser fulvescens $(18,19)$

Catostomus catostomus $(6,16)$

Catostomus commersoni $(9,10$,

13)

Coregonus clupeaformis $(2,4)$

Culaea inconstans $(9,10)$

Esox lucius $(5,6,9,10,14,15)$

Etheostoma exile $(1,9,14)$

Lota lota $(2,4,11,16)$

Moxostoma anisurum $(9,10)$

Notropis hudsonius (6)

Noturus flavis $(1,16)$

Perca flavescens $(1,2,4,6,7,8$,

$9,10,11,12,13,15,16,17)$

Percina caprodes $(1,9,10)$

Percopsis omiscomaycus $(1,9)$

Pungitius pungitius $(9,10)$

Salvelinus fontinalis (4)

Salvelinus namaycush $(3,4)$

Stizostedion vitreum $(1,6,15,17)$

Distribution: $\quad \mathrm{MB}, \mathrm{ON}, \mathrm{QC}$

Records: 1) Bangham \& Hunter 1939

(ON); 2) Bangham 1941 (ON); 3)

MacLulich 1943b (OIV); 4) Bangham \&

Venard 1946 (ON); 5) Worley \&

Bangham 1952 (QC); 6) Bangham 1955

(ON); 7) Tedla \& Fernando 1969a (ON);

8) Tedla 1969 (ON); 9) Dechtiar 1972a

(ON); 10) Dechtiar 1972b (ON); 11)

Tedla \& Fernando 1972 (ON); 12) 
Cannon 1973 (ON); 13) Collins \&

Dechtiar 1974 (ON); 14) Anthony 1983

(ON); 15) Dechtiar \& Christie 1988

(ON); 16) Dechtiar et al. 1988 (ON); 17)

Dechtiar \& Nepszy 1988 (ON); 18)

Choudhury et al. 1990 (MB); 19)

Choudhury \& Dick 1993 (MB)

\section{FAMILY POMPHORHYNCHIDAE}

**Pomphorhynchus bulbocolli Linkins in Van Cleave, 1919

Sites: intestine, mesenteries

Hosts: Acipenser fulvescens $(40,42,43$, $44,45,48)$

Carpiodes cyprimus $(13,14,32$,

$34,36,45$ )

Catostomus catostomus $(8,35,39)$

Catostomus commersoni $(1,2,3$,

$4,5,6,7,8,13,15,17,19,20,23,25$,

$26,27,31,33,34,35,36,37,45,48$ )

Catostomus macrocheilus $(8,19$,

24, 27, 29)

Coregonus artedii $(16,17,18,23)$

Coregonus clupeaformis $(16,17$,

$18,21,23,25,26,27,34)$

Esox lucius $(9,18,45)$

Hiodon tergisus $(14,22)$

Lota lota $(8,9,10,13,35)$

Moxostoma anisurum $(14,45)$

Moxostoma macrolepidotum (5,

14)

Notropis hudsonius $(9,35,45)$

Oncorhynchus clarki (8)

Oncorhynchus kisutch $(8,16,23$,

$25,27)$

Oncorhynchus mykiss $(8,19,24$,

26, 29, 34, 37)

Perca flavescens $(9,11,12,20$,
$30,46)$

Percina caprodes $(9,13,34,35)$

Percopsis omiscomaycus $(9,15$,

34, 35)

Phoximus eos $(2,48)$

Prosopium cylindraceum $(34,35)$

Prosopium williamsoni $(8,19,24$, 27, 28, 29)

Ptychocheilus oregonensis $(8,19$, 24, 27, 28, 29)

Pungitius pungitius $(17,23)$

Rhinichthys cataractae $(35,44)$

Richardsonius balteatus (8)

Salvelinus fontinalis $\mathrm{X}$ Salvelinus

namaycush $(15,20,34)$

Salvelinus namaycush $(16,17,23)$

Distribution: $\quad$ AB, BC, MB, ON, QC, SK

Records: 1) Bangham \& Hunter 1939

(ON); 2) Bangham 1941 (ON); 3)

Richardson 1942 (QC); 4) Bangham \&

Venard 1946 (ON); 5) Stewart-Hay

1951b (MB); 6) Stewart-Hay 1952b

(MB); 7) Stewart-Hay 1951g (MB); 8)

Bangham \& Adams 1954 (BC); 9)

Bangham 1955 (ON); 10) Fillion 1961

(AB - Lac Ste. Anne); 11) Tedla 1969

(ON); 12) Tedla \& Fernando 1972 (ON);

13) Dechtiar 1972a (ON); 14) Dechtiar

1972b (ON); 15) Collins \& Dechtiar 1974

(ON); 16) Leong \& Holmes 1974a (AB -

Cold Lake); 17) Leong 1975 (AB - Cold

Lake); 18) Watson 1977 (MB); 19)

Anonymous 1978 (BC); 20) Dechtiar \&

Berst 1978 (ON); 21) Pybus \& Samuel

1978 (AB - Wabamum Lake); 22) Glenn 1980 (MB); 23) Leong \& Holmes 1981

(AB - Cold Lake); 24) Anonymous 1981

(BC); 25) Drouin 1982 [AB - McGregor

Lake, Travers Reservoir ( $C$.

commersoni), Travers Reservoir (C.

clupeaformis)]; 26) McAllister \&

Mudry 1983 (AB - Ghost Lake, Tyrell 
Lake); 27) Arai \& Mudry 1983 (BC); 28) Anonymous 1983 (BC); 29) Anonymous 1984 (BC); 30) Poole \& Dick 1985 (MB); 31) Courtney et al. 1987 (BC); 32) Szalai \& Dick 1987a (MB); 33) Dechtiar \& Christie 1988 (ON); 34) Dechtiar et al. 1988 (ON); 35) Dechtiar \& Lawrie 1988 (ON); 36) Dechtiar \& Nepszy 1988 (ON); 37) Szalai \& Dick 1988 (MB); 38) Amedjo \& Holmes (AB - Tyrell Lake); 39) Dechtiar et al. 1989 (ON); 40) Choudhury et al. 1990 (SK); 41) Szalai \& Dick 1990 (SK); 42) Choudhury \& Dick 1991 (MB, SK); 43) Svanson et al. 1991 (MB); 44) Szalai et al. 1992 (MB); 45) Choudhury \& Dick 1993 (MB, SK); 46) Zelmer 1994 (AB - Burntstick Lake, Coal Lake, Fincastle Lake, Joker Lake, Pigeon Lake, Pine Lake, Sylvan Lake); 47) Choudhury \& Dick 1994 (MB); 48)

Dubois et al. 1996 (QC)

Pomphorhynchus sp.

Site: intestine

Hosts: Catostomus commersoni $(1,4)$ Coregonus clupeaformis $(2,3)$ Notropis hudsonius (3) Stizostedion vitreum (1)

Distribution: $\quad \mathrm{MB}$

Records: 1) Stewart-Hay 1951b; 2) Stewart-Hay 1951f; 3) Stewart-Hay 1951g; 4) Stewart-Hay $1952 b$

FAMILY RHADINORHYNCHIDAE

Rhadinorhynchus trachuri Harada, 1935

Synonym: Nipporhynchus trachuri
(Harada, 1935) Van Cleave and Lincicome, 1940

Site: intestine

Host: Oncorhynchus kisutch

Distribution: $\quad$ BC

Record: Anonymous 1981; Anonymous 1984

**Acanthocephala gen. sp.

Sites: coelom, intestine, mesenteries

Hosts: Acipenser fulvescens $(8,11)$

Catostomus commersoni (5)

Coregonus clupeaformis $(6,9,12)$

Esox lucius $(6,7)$

Lota lota (6)

Oncorhynchus mykiss (4)

Perca flavescens (3)

Salvelinus fontinalis $(1,2,4)$

Salvelinus namaycush $(9,10)$

Stizostedion canadense (3)

Stizostedion vitreum (3)

Distribution: $\quad$ AB, MB, NF, NT, ON, QC, SK

Records: 1) Ricker 1932 (ON); 2)

Richardson 1935 (QC); 3) Bangham \& Hunter 1939 (ON); 4) Frost 1940 (NF);

5) Stewart-Hay 1952b (MB); 6)

McTavish 1952 (MB); 7) McTavish 1953

(MB); 8) Harkness \& Dymond 1961

(ON); 9) McAllister \& Mudry 1983

[AB-Cold Lake (S. namaycush), Buck

Lake (C. clupeaformis)]; 10) Stewart \& Bernier 1983 (NT); 11) Choudhury \&

Dick 1991 (MB, SK)

PHYLUM ANNELIDA

CLASS HIRUDINEA 
ORDER GNATHBDELLIDA

FAMILY HIRUDINIDAE

Macrobdella decora (Say, 1824) Verrill, 1872

Site: body surface

Hosts: Acipenser fulvescens (2) Stizostedion vitreum (1)

Distribution: ON, SK

Records: 1) Moore 1922 (SK); 2) Moore 1924 (ON)

Mollibdella grandis (Verrill, 1874)

Richardson, 1969

Site: body surface

Host: Esox lucius

Distribution: ON

Record: Moore 1922

Percymoorensis marmorata (Say, 1824)

Richardson, 1969

Synonym: Haemopsis marmorata Say, 1824

Site: body surface

Host: Stizostedion vitreum

Distribution: SK

Records: Moore 1922; Madill 1988

ORDER RHYNCHOBDELLIDA

FAMILY GLOSSIPHONIDAE
Actinobdella inequianmulata Moore, 1901

Synonym: Actinobdella trianmulata Moore, 1924

Site: gill chamber

Hosts: Catostomus catostomus $(3,5,6,10$, $14,15)$

Catostomus commersoni $(1,2,4,6$, $7,8,9,10,11,12,13,14,15,16,17)$

Catostomus macrocheilus $(3,5)$

Distribution: BC, ON, SK

Records: 1) Bangham 1941 (ON); 2)

Bangham \& Venard 1946 (ON); 3)

Bangham \& Adams 1954 (BC); 4) Meyer

\& Moore 1954 (ON); 5) Bangham 1955

(ON); 6) Reed 1962 (SK); 7) Dechtiar 1972b (ON); 8) Molnar et al. 1974 (ON);

9) Lester \& Daniels 1976 (ON); 10)

Daniels \& Freeman 1976 (ON); 11)

Bower \& Woo 1977 (ON); 12) Chan 1980 (ON); 13) Dechtiar \& Christie 1988 (ON); 14) Dechtiar et al. 1988 (ON); 15) Dechtiar \& Lawrie 1988 (ON); 16) Jones \& Woo 1992a (ON); 17) Dubois et al. 1996 (QC)

Actinobdella sp.

Site: gill chamber

Hosts: Catostomus catostomus (2) Perca flavescens (1)

Distribution: ON, QC

Records: 1) Worley \& Bangham 1952

(QC); 2) Dechtiar et al. 1989 (ON)

Desserobdella phalera (Graf, 1899) Jones and Woo, 1990

Synonym: Clepsine phalera Graf, 1899 Site: body surface 
Hosts: Esox lucius

Gasterosteus aculeatus

Oncorhynchus mykiss

Perca flavescens

Stizostedion vitreum

Distribution: ON

Record: Jones \& Woo 1990

Placobdella montifera Moore, 1906

Site: body surface

Hosts: Acipenser fulvescens (4)

Catostomus commersoni $(2,3)$

Esox lucius $(2,3)$

Moxostoma macrolepidotum $(2,3)$

Perca flavescens $(1,2,3)$

Stizostedion vitreum $(2,3)$

Distribution: $\quad$ MB

Records: 1) Szalai \& Dick 1991a; 2)

Szalai \& Dick 1991b; 3) Szalai et al.

1992; 4) Choudhury \& Dick 1993

Placobdella parasitica (Say, 1824) Moore, 1901

Site: body surface

Host: Perca flavescens

Distribution: ON

Record: Ryerson 1915

Placobdella sp.

Site: body surface

Host: Acipenser fulvescens

Distribution: MB

Record: Choudhury et al. 1990
Site: body surface

Host: Acipenser fulvescens

Distribution: $\quad \mathrm{MB}$

Record: $\quad$ Choudhury \& Dick 1993

\section{FAMILY PISCICOLIDAE}

Cystobranchus mammilatus (Malm, 1863)

Johansson, 1896

Site: operculum

Host: Lota lota

Distribution: NT

Record: Meyer \& Roberts 1977

Cystobranchus meyeri Hayunga and Grey, 1976

Site: body surface

Host: Catostomus commersoni

Distribution: ON

Record: Klemm 1982

Cystobranchus verrilli Meyer, 1940

Site: gill chamber

Hosts: Lota lota $(1,2,3,4)$

Moxostoma anisurum (6)

Moxostoma macrolepidotum (5)

Distribution: $\quad$ AB, MB, ON

Records: 1) Meyer \& Moore 1954 (ON);

2) Leong 1975 (AB - Cold Lake); 3)

Leong \& Holmes 1981 (AB - Cold

Lake); 4) Dechtiar et al. 1989 (ON); 5)

Szalai \& Dick 1991 b (MB); 6) Szalai et

al. 1992 (MB)

Glossiphonidae gen. sp. 
Myzobdella lugubris Leidy, 1851

Synonyms: Illinobdella alba Meyer, 1940; Illinobdella moorei Meyer, 1940; Myzobdella moorei (Meyer, 1940) Meyer and Moore, 1954

Includes: Myzobdella alba Moore, 1940 of Dechtiar and Christie (1988)

Site: body surface

Hosts: Catostomus catostomus (3)

Catostomus commersoni (12)

Lota lota (8)

Moxostoma macrolepidotum (12)

Notropis hudsonius (12)

Perca flavescens $(1,2,10,11,12)$

Percina caprodes (12)

Percopsis omiscomaycus (12)

Stizostedion canadense (6)

Stizostedion vitreum $(4,5,6,9,12)$

Distribution: AB, MB, ON, SK

Records: 1) Meyer \& Moore 1954 (ON);

2) Bangham 1955 (ON); 3) Reed 1962

(SK); 4) Moore 1964 (AB - Bow River);

5) Dechtiar 1972b (ON); 6) Dechtiar

1972c (ON); 7) Appy \& Cone 1982

(ON); 8) Dechtiar \& Lawrie 1988 (ON);

9) Dechtiar \& Nepszy 1988 (ON); 10)

Dechtiar et al. 1988 (ON); 11) Szalai \&

Dick 1991a (MB); 12) Szalai \& Dick

$1991 b$ (MB)

Myzobdella sp.

Includes: Illinobdella sp.

Site: body surface

Hosts: Catostomus commersoni (12)

Esox lucius $(7,11)$

Etheostoma exile (5)

Oncorhynchus mykiss (4)

Perca flavescens $(1,2,3,6,7,9$,

10)
Percopsis omiscomaycus (8)

Ptychocheilus oregonensis (4)

Distribution: $\quad \mathrm{BC}, \mathrm{ON}, \mathrm{QC}$

Records: 1) Bangham 1941 (ON); 2)

Bangham \& Venard 1946 (ON); 3)

Worley \& Bangham 1952 (QC); 4)

Bangham \& Adams 1954 (BC); 5)

Bangham 1955 (ON); 6) Tedla 1969

(ON); 7) Dechtiar 1972a (ON); 8)

Dechtiar 1972b (ON); 9) Tedla \&

Fernando 1972 (ON); 10) Chan 1980

(ON); 11) Dechtiar et al. 1988 (ON);

12) Dechtiar \& Nepszy 1988 (ON)

Piscicola geometra (Linnaeus 1758) de

Blainville, 1818

Site: body surface

Host: Catostomus catostomus (2)

Catostomus commersoni (2)

Coregonus artedii (3)

Coregonus clupeaformis (3)

Esox lucius (2)

Perca flavescens (3)

Salvelinus namaycush (3)

Stizostedion vitreum $(1,2)$

Distribution: QC, SK

Records: 1) Rawson 1957a (SK); 2)

Rawson $1957 b$ (SK); 3) Madill 1988 (QC, SK)

Piscicola milneri (Verrill, 1874) Ryerson, 1915

Site: body surface

Hosts: Catostomus commersoni (7)

Coregonus artedii $(9,10)$

10)

Coregonus clupeaformis $(2,3,8,9$,

Esox lucius $(8,9,11)$ 
Lota lota (8)

Oncorhynchus mykiss (14)

Prosopium cylindraceum $(8,12)$

Salvelinus fontinalis $(13,14)$

Salvelinus namaycush $(1,4,5,6,8)$

Distribution: $\quad \mathbf{A B}, \mathrm{MB}, \mathrm{NT}, \mathrm{ON}, \mathrm{QC}$, YT

Records: 1) Ryerson 1915 (ON); 2) Moore 1924 (ON); 3) Meyer 1946 (ON);

4) Miller \& Kennedy 1948 (NT); 5) Moore \& Meyer 1951 (NT); 6) Meyer \& Moore 1954 (QC); 7) Moore 1964 (AB Cold Lake, Lower Waterton Lake, Upper Waterton Lake, Wabamum Lake); 8) Arthur et al. 1976 (YT); 9) Watson 1977 (MB); 10) Watson \& Dick 1979 (MB); 11) Watson \& Dick 1980 (MB); 12) Dechtiar \& Lawrie 1988 (ON); 13) Madill 1988 (ON)

Piscicola punctata (Verrill, 1871) Moore, 1912

Sites: body surface, gills

Hosts: Acipenser fulvescens (13)

Coregonus artedii (2)

Esox lucius (8)

Lota lota $(5,9)$

Perca flavescens $(2,5)$

Percina caprodes $(5,9)$

Percopsis omiscomaycus (12)

Ptychocheilus oregonensis (6)

Salvelinus fontinalis $(3,10,11$,

$12,14)$

Stizostedion canadense (1)

Stizostedion vitreum $(4,5,7,8)$

Distribution: $\quad$ AB, BC, NB, ON, SK

Records: 1) Moore 1924 (ON); 2)

Rawson 1930 (ON); 3) Ricker 1932

(ON); 4) Moore 1936 (ON); 5) Bangham

\& Hunter 1939 (ON); 6) Clemens et al.
1939 (BC); 7) Rawson 1957a (SK); 8)

Oliver1960 (SK); 9) Dechtiar 1972a

(ON); 10) Frimeth 1987a (NB); 11)

Frimeth $1987 \mathrm{~b}(\mathrm{NB})$; 12) Dechtiar et al.

1988 (ON); 13) Dechtiar \& Lawrie 1988

(ON); 14) Madill 1988 (AB - Tay River)

Piscicola salmositica Meyer, 1946

Sites: body surface, gill chamber

Hosts: Catostomus macrocheilus $(2,3)$

Oncorhynchus kisutch (1)

Distribution: $\quad \mathrm{BC}$

Records: $\quad$ 1) Becker \& Katz 1965a; 2)

Anonymous 1981; 3) Anonymous 1984

Piscicola sp.

Site: body surface

Hosts: Acipenser fulvescens (8)

Catostomus macrocheilus (6)

Margariscus margarita (7)

Perca flavescens $(1,2,3,5)$

Prosopium williamsoni (6)

Stizostedion vitreum (4)

Distribution: $\quad \mathrm{BC}, \mathrm{MB}, \mathrm{ON}$

Records: 1) Tedla 1969 (ON); 2) Tedla

\& Fernando 1969a (ON); 3) Tedla \&

Fernando 1972 (ON); 4) Anthony 1978b

(ON); 5) Chan 1980 (ON); 6) Anonymous

1981 (BC); 7) Dechtiar et al. 1989 (ON);

8) Choudhury et al. 1990 (MB)

Piscicolaria reducta Meyer, 1940

Site: body surface

Host: Percina caprodes

Distribution: ON

Record: $\quad$ Appy \& Cone 1982 
Hirudinea gen. sp.

Sites: body surface, gill chamber, gills

Hosts: Acipenser fulvescens (4) Catostomus macrocheilus (3)

Lota lota (1)

Prosopium williamsoni (3)

Salvelinus fontinalis (2)

Distribution: $\quad \mathrm{BC}, \mathrm{MB}, \mathrm{NF}, \mathrm{SK}$

Records: 1) McTavish 1952 (MB); 2)

Sandeman \& Pippy 1967 (NF); 3)

Anonymous 1978 (BC); 4) Choudhury \&

Dick 1991 (MB, SK)

PHYLUM MOLLUSCA

CLASS BIVALVIA

ORDER EULAMELLIBRANCHIA

SUPERFAMILY UNIONACEA

FAMILY UNIONIDAE

*Anodonta cataracta Say, 1817

Includes: Anodonta cataracta cataracta

Say, 1817; Anodonta cataracta fragilis

Lamarck, 1819

Sites: fins, gills, opercula

Hosts: Catostomus commersoni (1)

Gasterosteus aculeatus $(1,2)$

Salvelinus fontinalis (2)

Distribution: NF, NS

Records: 1) Wiles 1975 (NS); 2)
Threlfall 1986 (NF)

*Anodonta grandis simpsonia Lea, 1861

Sites: body surface, fins, gills

Host: Perca flavescens

Distribution: $\mathbf{A B}$

Records: Jansen 1991 (AB - Narrow

Lake); Jansen \& Hanson 1991 (AB -

Narrow Lake); Nelson \& Paetz 1992

(AB - not specified)

*Anodonta implicata Say, 1829

Site: gills

Hosts: Catostomus commersoni Gasterosteus aculeatus

Distribution: NS

Record: Wiles 1975

*Anodonta sp.

Site: gills

Hosts: Gasterosteus aculeatus (4)

Perca flavescens $(1,2,3)$

Ptychocheilus oregonensis (5)

Pungitius pungitius (4)

Distribution: BC, NS, ON

Records: 1) Tedla \& Fernando 1969d (ON); 2) Tedla 1969 (ON); 3) Tedla \& Fernando 1972 (ON); 4) Wiles 1975 (NS); 5) Anonymous 1984 (BC)

*Anodontoides ferrussacianus Lea, 1834

Sites: fins, gills

Hosts: Catostomus commersoni

Hybognathus hankinsoni 
Perca flavescens

Phoximus eos

Pimephales promelas

Distribution: $\mathrm{ON}$

Record: Kakonge 1972

*Elliptio complanata (Lightfoot, 1786)

Rafinesque, 1819

Site: gills

Hosts: Catostomus commersoni (5) Gasterosteus aculeatus (5) Perca flavescens $(1,2,3,4)$

Distribution: NS, ON

Records: 1) Tedla \& Fernando 1969a

(ON); 2) Tedla 1969 (ON); 3) Tedla \& Fernando 1970a (ON); 4) Tedla \& Fernando 1972 (ON); 5) Wiles 1975 (NS)

*Lampsilis radiata radiata (Gmelin, 1791) Rafinesque, 1820

Includes: Lampsilis radiata siliquodes (Barnes, 1823) of Tedla and Fernando (1969)

Site: gills

Hosts: Catostomus commersoni (6) Gasterosteus aculeatus (6) Perca flavescens $(1,2,3,4,5)$

Distribution: NS, ON

Records: $\quad$ 1) Tedla \& Fernando 1969a

(ON); 2) Tedla 1969 (ON); 3) Tedla \& Fernando 1969d (ON); 4) Tedla \& Fernando 1970a (ON); 5) Tedla \&

Fernando 1972 (ON); 6) Wiles 1975 (NS)

${ }^{*}$ Lampsilis sp.

Sites: fins, gills
Hosts: Catostomus commersoni

Distribution: ON Perca flavescens

Record: Kakonge 1972

* Lasmigona compressa (Lea, 1829)

Sites: fins, gills

Hosts: Culaea inconstans

Distribution: ON

Record: Kakonge 1972

*Unionidae gen. sp.

Sites: body surface, fins, gills

Hosts: Carpiodes cyprinus $(21,25,28)$

Catostomus catostomus $(5,23$,

26)

$23,25,28$ )

Catostomus commersoni $(5,7,22$,

Coregonus artedii (26)

Coregonus clupeaformis $(1,15)$

Cottus ricei (23)

Couesius plumbeus $(4,23)$

Esox lucius $(13,16,19,22,28)$

Gasterosteus aculeatus $(4,12,22)$

Hybognathus hankinsoni (10)

Lota lota $(7,23)$

Moxostoma anisurum (28)

Notropis atherinoides (8)

Oncorhynchus mykiss (4)

Perca flavescens $(3,5,6,7,9,14$,

22, 23, 24, 25, 27, 28;

Percopsis omiscomaycus $(22,24)$

Phoxinus eos (26)

Phoxinus neogaeus (23)

Pimephales promelas $(9,10,17)$

Prosopium cylindraceum (26)

Ptychocheilus oregonensis (4) 
Pungitius pungitius (7)

Richardsonius balteatus (4)

Salvelinus fontinalis $(18,20,26)$

Salvelinus fontinalis $\mathrm{X}$ Salvelinus namaycush (26)

Stizostedion canadense $(2,7)$

$25,28)$

Stizostedion vitreum $(5,7,14,22$,

Distribution: $\quad \mathbf{A B}, \mathrm{BC}, \mathrm{MB}, \mathrm{NF}, \mathrm{NS}, \mathrm{ON}$, QC

Records: 1) Hart 1930 (ON); 2)

Bangham \& Hunter 1939 (ON); 3)

Bangham 1941 (ON); 4) Bangham \&

Adarns 1954 (BC); 5) Bangham 1955

(ON); 6) Tedla \& Fernando 1969a (ON);

7) Dechtiar 1972a (ON); 8) Dechtiar 1972b (ON); 9) Kakonge 1972 (ON); 10) Molnar et al. 1974 (ON); 11) Wiles 1975 (NS); 12) Lester 1975 (BC); 13) Watson 1977 (MB); 14) Anthony 1978b (ON);

15) Pybus \& Samuel 1978 [AB - Cache Lake (P. flavescens), Blue Lake ( $C$. clupeaformis)]; 16) Watson \& Dick 1980 (MB); 17) Chan 1980 (ON); 18) Black 1981a (QC); 19) Anthony 1983 (ON); 20) Cone \& Ryan 1984 (NF); 21) Szalai \& Dick 1987a (MB); 22) Dechtiar \& Christie 1988 (ON); 23) Dechtiar et al. 1988 (ON); 24) Dechtiar \& Lawrie 1988 (ON); 25) Dechtiar \& Nepszy 1988 (ON); 26) Dechtiar et al. 1989 (ON); 27) Szalai \& Dick 1991a (MB); 28) Szalai et al. 1992 (MB)

PHYLUM ARTHROPODA

CLASS CRUSTACEA

SUBCLASS BRANCHIURA
ORDER ARGULOIDEA

FAMILY ARGULIDAE

Argulus appendiculosus Wilson, 1907

Site: body surface

Hosts: Carpiodes cyprinus $(3,4)$

Catostomus commersoni $(3,4)$

Coregonus artedii $(3,4)$

Esox lucius $(3,4)$

Hiodon alosoides (1)

Notropis atherinoides $(3,4)$

Percopsis omiscomaycus (4)

Stizostedion canadense (2)

Stizostedion vitreum $(3,4)$

Distribution: MB, ON, SK

Records: 1) Reed 1962 (SK); 2) Dechtiar

1972a (ON); 3) Szalai \& Dick 1991b

(MB); 4) Szalai et al. 1992 (MB)

Argulus biramosus Bere, 1931

Site: fins

Hosts: Catostomus commersoni Coregonus clupeaformis

Distribution: $\mathbf{A B}$

Record: Clifford 1991 (AB - South

Saskatchewan River drainage)

Argulus catostomi Dana and Herrick, 1837

Site: fins

Hosts: Carpiodes cyprinus (5)

Catostomus catostomus (4)

Catostomus commersoni $(1,3,5)$

Culaea inconstans (2)

Etheostoma exile (1) 
Moxostoma macrolepidotum (4)

Phoxinus neogaeus (4)

Distribution: ON

Records: 1) Dechtiar 1972a; 2) Kakonge 1972; 3) Dechtiar \& Christie 1988; 4)

Dechtiar et al. 1988; 5) Dechtiar \&

Nepszy 1988

Argulus stizostethi Kellicott, 1880

Synonym: Argulus canadensis Wilson, 1916

Site: body surface

Hosts: Acipsenser fulvescens $(1,6)$

Catostomus commersoni (3)

Coregonus artedii $(5,9,10,11)$

Coregonus clupeaformis $(5,10,11)$

Esox lucius $(10,12)$

Gasterosteus aculeatus $(8,13,14$,

$15,16,17,18)$

Notropis hudsonius (9)

Perca flavescens $(2,9)$

Pungitius pungitius $(13,14,15,17)$

Salvelinus fontinalis $(2,7)$

Stizostedion vitreum $(3,4,5,6,9)$

Distribution: $\quad$ MB, NF, ON, QC, SK

Records: 1) Wilson 1916 (ON); 2)

Wilson 1936 (NS); 3) Stewart-Hay 1951b

(MB); 4) Rawson 1957a (SK); 5) Rawscn

$1957 \mathrm{~b}$ (SK); 6) Harkness \& Dymond

1961 (ON); 7) Sandeman \& Pippy 1967

(NF); 8) Threlfall 1968 (NF); 9) Dechtiar

1972a (ON); 10) Watson 1977 (MB); 11)

Watson \& Dick 1979 (MB); 12) Watson

\& Dick 1980 (MB); 13) Poulin \&

Fitzgerald 1987 (QC); 14) Poulin \&

Fitzgerald 1988 (QC); 15) Poulin \&

Fitzgerald 1989a (QC); 16) Poulin \&

Fitzgerald 1989b (QC); 17) Poulin \&

Fitzgerald 1989c (QC); 18) Poulin \&

Fitzgerald 1989d (QC)
Argulus versicolor Wilson, 1902

Site: fins

Hosts: Percopsis omiscomaycus Stizostedion vitreum

Distribution: ON

Record: Dechtiar 1972a

Argulus sp.

Site: body surface

Hosts: Coregonus artedii (1)

Esox lucius (2)

Percopsis omiscomaycus (1)

Distribution: ON

Records: 1) Bangham 1955; 2) Dechtiar et al. 1988

SUBCLASS ENTOMOSTRACA

ORDER COPEPODA

SUBORDER CYCLOPOIDA

FAMILY LERNAEIDAE

Lernaea catostomi (Krøyer, 1863) Wilson, 1914

Site: penetrating into musculature from body surface

Hosts: Catostomus catostomus

Catostomus commersoni

Moxostoma macrolepidotum

Distribution: SK

Record: Reed 1962 
Lernaea cyprinacea Linneaus, 1758

Site: penetrating musculature from body surface

Hosts: Catostomus commersoni $(2,3,4)$ Culaea inconstans (1)

Distribution: $\mathrm{MB}, \mathrm{ON}$

Records: 1) Kakonge 1972 (ON); 2)

Lester \& Daniels 1976 (ON); 3) Szalai \& Dick 1991b (MB); 4) Szalai et al. 1992 (MB)

SUBORDER POECILOSTOMATOIDEA

FAMILY ERGASILIDAE

Ergasilus auritus Markevich, 1940

Synonym: Markewitschia aurita

(Markevich, 1940)

Sites: body surface, gills

Hosts: Coregonus artedii $(7,8,10)$

Coregonus clupeaformis $(7,8,10)$

Gasterosteus aculeatus (1, 2, 3, 4,

$5,9)$

Oncorhynchus kisutch $(7,8,10)$

Pungitius pungitius $(8,10)$

Salvelinus fontinalis (6)

Salvelinus namaycush $(7,8,10)$

Distribution: $\quad$ AB, BC, LB, NF, ON, QC

Records: 1) Markevich 1940 (BC); 2)

Markevich 1956 (BC); 3) Roberts 1963

(BC); 4) Hanek \& Threlfall 1970a (LB);

5) Hanek \& Threlfall 1970c (NF); 6)

Hanek \& Molnar 1974 (QC); 7) Leong \&

Holmes 1974a (AB - Cold Lake); 8)

Leong 1975 (AB - Cold Lake); 9) Lester 1975 (BC); 10) Leong \& Holmes 1981

(AB - Cold Lake)
Ergasilus caeruleus Wilson, 1911

Site: gills

Hosts: Carpiodes cyprinus $(6,19)$

Catostomus catostomus $(2,4,5,7$,

$9,10,11,12,13,14,15,17,18,20)$

Catostomus commersoni $(2,4,5,7$,

$8,9,16,17,18,19)$

Coregonus artedii (2)

Coregonus clupeaformis $(3,8)$

Esox lucius (18)

Lota lota $(2,3)$

Moxostoma anisurum (7)

Moxostoma macrolepidotum (17)

Perca flavescens $(4,7,8,9,17,18$, 21, 22)

Percopsis omiscomaycus $(4,6,8$, $17,18)$

Prosopium cylindraceum (3)

Rhinichthys cataractae (17)

Salvelinus fontinalis X Salvelinus

namaycush $(8,9)$

Stizostedion canadense $(1,7)$

Stizostedion vitreum $(1,4,7,16$, $17,19)$

Distribution: $\quad$ AB, BC, LB, ON

Records: 1) Bangham \& Hunter 1939

(ON); 2) Bangham 1941 (ON); 3)

Bangham \& Venard 1946 (ON); 4)

Bangham 1955 (ON); 5) Threlfall \&

Hanek 1970a (LB); 6) Dechtiar 1972b

(ON); 7) Dechtiar 1972a (ON); 8) Collins

\& Dechtiar 1974 (ON); 9) Dechtiar \&

Berst 1978 (ON); 10) Chinniah \&

Threlfall 1978 (LB); 11) Hanek \&

Fernando 1978a (ON); 12) Hanek \&

Fernando 1978b (ON); 13) Hanek \&

Fernando 1978d (ON); 14) Hanek \&

Fernando 1978e (ON); 15) Hanek \&

Fernando 1978f(ON); 16) Dechtiar \& Christie 1988 (ON); 17) Dechtiar et al. 1988 (ON); 18) Dechtiar \& Lawrie 1988 
(ON); 19) Dechtiar \& Nepszy 1988 (ON);

20) Dechtiar et al. 1989 (ON); 21)

Zelmer 1994 (AB - Garner Lake); 22)

Zelmer \& Arai 1998 (AB - Garner

Lake)

Ergasilus celestis Mueller, 1936

Synonym: Ergasilus osborni Tidd and Bangham, 1945

Site: gills

Host: Lota lota

Distribution: ON

Records: $\quad$ Tidd \& Bangham 1945;

Bangham \& Venard 1946; Roberts 1970;

Dechtiar 1972a; Dechtiar et al. 1988;

Dechtiar et al. 1989

Ergasilus centrachidarum Wright, 1882

Site: gills

Hosts: Catostomus catostomus (3)

Perca flavescens (1)

Stizostedion canadense (2)

Stizostedion vitreum $(2,4)$

Distribution: ON

Records: 1) Wright 1882 ; 2) Bangham \&

Hunter 1939; 3) Roberts 1970; 4)

Dechtiar 1972a

Ergasilus cyprinaceus Rogers, 1969

Site: gills

Host: Pimephales promelas

Distribution: ON

Records: $\quad$ Molnar et al. 1974; Chan 1980

Ergasilus elongatus Wilson, 1916
Site: gills

Hosts: Coregonus clupeaformis Salvelimus namaycush

Distribution: $\quad$ ON

Record: Dechtiar et al. 1989

Ergasilus labracis Krøyer, 1864

Includes: Ergasilus funduli Krøyer, 1863 of Fréchet et al. (1983)

Site: gills

Host: Salvelinus fontinalis

Distribution: NB

Records: Frimeth 1987a; Frimeth 1987b

Ergasilus lizae Krøyer, 1863

Site: gills

Host: Carpiodes cyprinus

Distribution: $\quad \mathrm{MB}$

Records: Szalai \& Dick 1987a; Szalai et al. 1992

Ergasilus luciopercarum Henderson, 1926

Synonym: Ergasilus confusus Bere, 1926

Site: gills

Hosts: Coregonus artedii (23)

Esox lucius $(12,15)$

Perca flavescens $(3,4,5,6,7,8$, $10,13,14,17,18,19,20,21,22,23)$

Percopsis omiscomaycus (14)

Prosopium cylindraceum (11)

Salvelinus fontinalis (2)

Stizostedion canadense (14)

Stizostedion vitreum $(1,9,13,14$, $16,17,18,19,20,21,23)$

Distribution: $\quad \mathrm{LB}, \mathrm{MB}, \mathrm{NF}, \mathrm{ON}, \mathrm{QC}$ 
Records: 1) Henderson 1926 (QC); 2) Sandeman \& Pippy 1967 (NF); 3) Tedla \& Fernando 1969a (ON); 4) Tedla \& Fernando 1969b (ON); 5) Tedla \& Fernando 1969d (ON); 6) Tedla 1969 (ON); 7) Tedla \& Fernando 1970a (ON); 8) Tedla \& Fernando 1970c (ON); 9) Roberts 1970 (ON); 10) Tedla \& Fernando 1972 (ON); 11) Hicks \& Threlfall 1973 (LB); 12) Watson 1977 (MB); 13) Anthony 1978b (ON); 14) Lubinsky \& Loch 1979 (MB); 15) Watson \& Dick 1980 (MB); 16) Kinnis \& Curtis 1981 (QC); 17) Poole \& Dick 1985 (MB); 18) Dechtiar \& Christie 1988 (ON); 19) Dechtiar et al. 1988 (ON); 20) Dechtiar \& Lawrie 1988 (ON); 21) Dechtiar \& Nepszy 1988 (ON); 22) Szalai \& Dick 1991a (MB); 23) Szalai et al. 1992 (MB)

Ergasilus nerkae Roberts 1963

Includes: Ergasilus caeruleus of Bangham and Adams 1954 (partim); Ergasilus sp. of Bangham and Adams (1954) (partim)

Site: gills

Hosts: Catostomus catostomus $(1,2,3,10$, $13,14)$

Catostomus commersoni $(8,12)$

Catostomus macrocheilus $(1,2,3$, $8,12)$

Coregonus artedii $(4,5,7,8,9,11)$ Coregonus clupeaformis $(4,5,7,8$, $9,11,12)$

Couesius plumbeus $(1,2)$

Gasterosteus aculeatus (2)

Hiodon alosoides (15)

Lota lota (1)

Oncorhynchus kisutch $(4,5,11)$
Oncorhynchus mykiss $(1,2,3,6,8$, 12, 13)

Prosopium williamsoni $(1,2,3)$

Ptychocheilus oregonensis (1, 3,

12)

Richardsonius balteatus $(1,2,3)$

Salvelinus namaycush $(4,5,6,11)$

Thymallus arcticus $(8,12)$

Distribution: $\quad \mathbf{A B}, \mathrm{BC}, \mathrm{MB}, \mathrm{ON}$

Records: 1) Bangham \& Adams 1954

(BC); 2) Roberts 1963 (BC); 3) Roberts

1970 (BC); 4) Leong \& Holmes 1974a

(AB - Cold Lake); 5) Leong 1975 (AB -

Cold Lake); 6) Mudry \& Anderson

1977 (AB - Pyramid Lake); 7) Watson

1977 (MB); 8) Anonymous 1978 (BC); 9)

Watson \& Dick 1979 (MB); 10)

Anonymous 1981 (BC); 11) Leong \&

Holmes 1981 (AB - Cold Lake); 12)

Arai \& Mudry 1983 (BC); 13)

Anonymous 1984 (BC); 14) Courtney

1987 (ON); 15) Szalai et al. 1992 (MB)

Ergasilus turgidus Fraser, 1920

Site: gills

Host: Gasterosteus aculeatus

Distribution: $\quad$ BC

Record: Carl 1937

Ergasilus versicolor Wilson, 1911

Synonym: Ergasilus elegans Wilson, 1916

Site: gills

Hosts: Catostomus commersoni (5)

Culaea inconstans $(3,4)$

Moxostoma anisurum (5)

Noturus flavus $(1,2,3,4)$

Distribution: $\quad \mathrm{MB}, \mathrm{ON}$ 
Records: 1) Bangham \& Hunter 1939

(ON); 2) Dechtiar 1972a (ON); 3)

Dechtiar \& Nepszy 1988 (ON); 4)

Dechtiar et al. 1988 (ON); 5) Szalai et al. 1992 (MB)

Ergasilus sp.

Site: gills

Hosts: Carpiodes cyprinus (13)

Catostomus catostomus $(9,10)$

Catostomus commersoni $(9,10,16)$

Coregonus artedii $(7,8,18)$

Coregonus clupeaformis $(1,16)$

Cottus ricei (13)

Couesius plumbeus (13)

Culaea inconstans (16)

Gasterosteus aculeatus (12)

Hiodon alosoides (5)

Notropis hudsonius $(4,8,14)$

Perca flavescens $(3,4)$

Prosopium cylindraceum (1)

Pungitius pungitius $(4,13)$

Salvelinus fontinalis $(6,11,17)$

Stizostedion vitreum $(2,3)$

Distribution: $\quad \mathbf{A B}, \mathrm{MB}, \mathrm{NF}, \mathrm{ON}$

Records: 1) Bangham 1941 (ON); 2)

McLeod 1943 (MB); 3) McLeod 1944

(MB); 4) Stewart-Hay 1952b (MB); 5)

Kennedy \& Sprules 1967 (MB); 6)

Threlfä.i \& Hanek 1970c (NF); 7) Paetz

\& Nelson 1970 (AB - Lac la Biche); 8)

Dechtiar 1972a (ON); 9) Leong 1975

(AB - Cold Lake); 10) Leong \& Holmes

1981 (AB - Cold Lake); 11) Cone \&

Ryan 1984 (NF); 12) Dechtiar \& Christie

1988 (ON); 13) Dechtiar et al. 1988

(ON); 14) Dechtiar \& Lawrie 1988 (ON);

15) Stock 1988 (AB - Jarvis Lake); 16)

Dechtiar et al. 1989 (ON); 17)

Marcogliese \& Cone 1991b (NF); 18)
Nelson \& Paetz 1992 (AB - unspecified)

Therestina gasterostei (Pagenstecher, 1861) Norman, 1905

Sites: gill chamber, gills

Hosts: Gasterosteus aculeatus (1, 2, 3, 5, $6,8)$

Pungitius pungitius $(4,7,8,9,10)$

Distribution: $\quad$ BC, LB, NF, NS, QC

Records: 1) Threlfall 1968 (NF); 2)

Hanek \& Threlfall 1969a (NF); 3) Hanek

\& Threlfall 1970a (LB, NF); 4) Hanek \&

Threlfall 1970b (LB); 5) Hanek \& Molnar 1974 (QC); 6) Lester 1975 (BC); 7)

Dickinson \& Threlfall 1976 (NF); 8)

Poulin \& Fitzgerald 1987 (QC); 9)

Marcogliese 1992 (NS); 10) Marcogliese 1995 (NS)

\section{SUBORDER SIPHONOSTOMATOIDEA}

\section{FAMILY LERNAEOPODIDAE}

Achtheres pimelodi Krøyer, 1863

Synonyms: Achtheres ambloplitis

Kellicott, 1880; Achtheres micropteri

Wright, 1882

Sites: buccal cavity, gill chamber, gills

Hosts: Coregonus clupeaformis (1) Perca flavescens (2)

Distribution: ON

Records: 1) Dechtiar 1972b; 2)

Dechtiar \& Nepszy 1988

Achtheres sp. 
Site: gills

Host: Prosopium williamsoni

Distribution: $\mathbf{A B}$

Record: Thompson 1974 (AB - Sheep River)

Coregonicola orientalis Markevich and Bauer, 1950

Site: penetrating musculature from body surface

Host: Coregonus clupeaformis

Distribution: NT

Record: $\quad$ Stewart \& Bernier 1983

\section{Coregonicola sp.}

Site: penetrating musculature from body surface

Hosts: Coregonus clupeaformis (2) Prosopium williamsoni $(1,3)$

Distribution: $\quad$ AB, NT

Records: 1) Paetz \& Nelson 1970 (AB not specified); 2) Reist et al. 1987 (NT);

3) Nelson \& Paetz 1992 (AB - not specified)

Salmonicola californiensis (Dana, 1852)

Wilson, 1915

Synonyms: Salmincola bicauliculata (Wilson, 1909); Salmincola carpenteri (Packard, 1874); Salmincola falculata (Wilson, 1908)

Sites: body surface, gill chamber, gills

Hosts: Oncorhynchus clarki (2) Oncorhynchus kisutch $(5,6)$ Oncorhynchus mykiss $(2,3,4,5$, $6,7)$
Prosopium williamsoni (5)

Salvelinus malma $(1,2)$

Salvelinus namaycush $(4,6)$

Distribution: $\quad$ BC, NT

Records: 1) Wilson 1920 (NT); 2) Bere 1930b (BC); 3) Bell \& Margolis 1976 (BC); 4) Anonymous 1978 (BC); 5) Anonymous 1981 (BC); 6) Arai \& Mudry 1983 (BC); 7) Anonymous 1984 (BC)

Salmincola carpionis (Krøyer, 1837) Wilson, 1915

Synonyms: Salmincola giber (Wilson, 1908); Salmincola salvelini Richardson, 1938

Sites: body surface, branchial chamber, gills

Hosts: Salvelinus fontinalis $(2,3)$ Salvelinus malma (1)

Distribution: NT, QC

Records: 1) Wilson 1920 (NT); 2) Richardson 1938 (QC); 3) Kabata 1969 (QC)

Salmincola coregonorum (Kessler, 1868) Kabata, 1969

Site: gills

Host: Coregonus clupeaformis

Distribution: LB

Record: Chinniah \& Threlfall 1978

Salmonicola corpulentus (Kellicott, 1880) Kabata, 1988

Synonyms: Achtheres corpulentus Kellicott, 1880; Salmonicola extumescens (Gadd, 1901) Wilson, 1915; Salmincola 
inermis (Wilson, 1911)

Sites: branchial chamber, gills

Hosts: Coregonus artedii $(2,5,18,19)$

Coregonus clupeaformis $(1,2,4$,

$5,7,9,10,11,12,13,14,15,17,20)$

Coregonus sp. (6)

Prosopium cylindraceum (18,

19)

Distribution: $\quad \mathbf{A B}, \mathrm{LB}, \mathrm{MB}, \mathrm{NT}, \mathrm{ON}$, QC

Records: 1) Hart 1931 (ON); 2)

Pritchard 1931 (ON); 3) Miller 1945e

(NT); 4) Rawson 1951 (NT); 5) Bangham

1955 (ON); 6) Kabata 1969 (NT, ON,

QC); 7) Dechtiar 1972a (ON); 8) Dechtiar

1972b (ON); 9) Leong \& Holmes 1974a

(AB - Cold Lake); 10) Leong 1975 (AB

- Cold Lake); 11) Watson 1977 (MB);

12) Chinniah \& Threlfall 1978 (LB); 13)

Watson \& Dick 1979 (MB); 14) Leong \&

Holmes 1981 (AB - Cold Lake); 15)

Stewart \& Bernier 1983 (NT); 16)

Stewart \& Bernier 1984 (NT); 17)

Dechtiar \& Christie 1988 (ON); 18)

Dechtiar et al. 1988 (ON); 19) Dechtiar \&

Lawrie 1988 (ON); 20) Hobbs (undated)

(AB - Lesser Slave Lake)

Salmincola edwardsi (Olsson, 1869) Wilson, 1915

Synonyms: Salmincola exsanguinata Sandeman and Pippy, 1967; Lernaeopoda arcturi Miers, 1877; Lernaeopoda edwardsi (Olsson, 1869)

Sites: body surface, branchial chamber, gills

Hosts: Oncorhynchus clarki (9)

Oncorhynchus mykiss $(6,9)$

Prosopium williamsoni $(18,23)$

Salvelinus fontinalis $(1,2,3,4$,
$5,6,7,8,10,11,12,13,14,16,17,19$, $20,21,24,25,28,29,30,31,32,33,34$, $35,36,37,38,39,40,41$ )

Salvelinus malma $(9,11,18,23)$

Salvelinus namaycush $(15,18$,

$22,23,26,27)$

Distribution: BC, EXP, LB, MB, NB, NF, NS, NT, ON, PE, QC, YT

Records: 1)Wright 1882 (QC); 2) Bere 1930a (NB); 3) Ricker 1932 (ON); 4)

Savage 1935 (ON); 5) Richardson 1935 (QC); 6) Frost 1940 (NF); 7) MacLulich 1943b (ON); 8) Kooyman 1951 (MB); 9)

Bangham \& Adams 1954 (BC); 10)

Sandeman \& Pippy 1967 (NF); 11)

Kabata 1969 (BC, NF, ON); 12) Hicks \& Threlfall 1973 (LB); 13) Hanek \& Molnar 1974 (QC); 14) Hare \& Frantsi 1974 (NS, PE); 15) Arthur et al. 1976 (YT); 16) Gaboury 1978 (MB); 17) Chinniah \& Threlfall 1978 (LB); 18) Anonymous 1978 (BC); 19) Black 1981a (QC); 20) Black 1982b (ON); 21) Black 1982a (ON); 22) Stewart \& Bernier 1982 (NT); 23) Arai \& Mudry 1983 (BC); 24) Cone \& Ryan 1984 (NF); 25) Black et al. 1983 (QC); 26) Stewart \& Bernier 1983 (NT); 27) Stewart \& Bernier 1984 (NT); 28) Frimeth 1987a (NB); 29) Frimeth 1987b (NB); 30) Conley \& Curtis 1988 (QC); 31) Dechtiar et al. 1988 (ON); 32) Albert \& Curtis 1988 (QC); 33) Poulin et al. 1989 (QC); 34) Poulin et al. 1990a (QC); 35) Poulin et al. 1990b (QC); 36) Poulin et al. 1991 (EXP, QC); 37) Marcogliese \& Cone 1991b (NF); 38) Conley \& Curtis 1992 (QC); 39) Conley \& Curtis 1993 (QC); 40) Conley \& Curtis 1994 (QC); 41) Dubois et al. 1996 (QC)

Salmincola extensus (Kessler, 1868) 
Kabata, 1969

Synonyms: Salmincola wisconsinensis

Tidd and Bangham 1945; Achtheres coregoni Baumann, 1911

Site: body surface

Hosts: Coregonus artedii $(3,6,8,10,13)$ Coregonus clupeaformis $(1,4,5$,

$6,7,8,10,13)$

Esox lucius $(8,11)$

Prosopium cylindraceum $(2,3)$

Prosopium williamsoni $(2,9,12$,

$14,15)$

Distribution: $\quad$ AB, BC, LB, MB, ON

Records: 1) Hart 1931 (ON); 2)

Bangham \& Adams 1954 (BC); 3)

Bangham 1955 (ON); 4) Hicks \&

Threlfall 1973 (LB); 5) Leong \& Holmes

1974a (AB - Cold Lake); 6) Leong 1975

(AB - Cold Lake); 7) Arthur et al. 1976

(YT); 8) Watson 1977 (MB); 9)

Anonymous 1978 (BC); 10) Watson \&

Dick 1979 (MB); 11) Watson \& Dick 1980 (MB); 12) Anonymous 1981 (BC);

13) Leong \& Holmes 1981 (AB - Cold

Lake); 14) Arai \& Mudry 1983 (BC); 15)

Anonymous 1984 (BC)

Salmincola lotae (Olsson, 1877) Wilson, 1915

Site: gills

Host: Lota lota

Distribution: NT

Record: Stewart \& Bernier 1983

Salmincola siscowet (Smith, 1874) Wilson 1915

Sites: body surface, gills
Hosts: Salvelinus fontinalis X Salvelinus namaycush (12)

Salvelimus malma (1)

Salvelinus namaycush $(1,2,3,4$,

$5,6,7,8,9,10,11,12$ )

Distribution: $\quad$ BC, LB, NT, ON, QC, SK

Records: 1) Wilson 1920 (NT); 2)

MacLulich 1943b (ON); 3) Miller 1945e

(NT); 4) Miller \& Kennedy 1948 (NT); 5)

Rawson 1951 (NT); 6) Rawson 1961

(SK); 7) Kabata 1969 (QC); 8) Dechtiar 1972a (ON); 9) Chinniah \& Threlfall 1978

(LB); 10) Anonymous 1984 (BC); 11)

Dechtiar \& Lawrie 1988 (ON); 12)

Anderson 1993 (ON)

Salmincola thymalli (Kessler, 1868) Wilson, 1915

Site: gills

Hosts: Coregonus clupeaformis (11)

Prosopium cylindraceum $(5,12)$

Prosopium williamsoni $(7,8,9,10$,

11)

$8,10)$

Thymallus arcticus $(1,2,3,4,6,7$,

Distribution: $\quad$ BC, LB, NT, ON, YT

Records: 1) Miller 1945e (NT); 2) Miller

1946a (NT); 3) Rawson 1951 (NT); 4)

Kabata 1969 (NT); 5) Hicks \& Threlfall 1973 (LB); 6) Arthur et al. 1976 (YT); 7)

Anonymous 1978 (BC); 8) Anonymous 1981 (BC); 9) Anonymous 1983 (BC);

10) Arai \& Mudry 1983 (BC); 11)

Anonymous 1984 (BC); 12) Dechtiar et

al. $1989(\mathrm{ON})$

Salmincola sp.

Sites: body surface, buccal cavity, gills 
Hosts: Coregomus clupeaformis (3)

Prosopium cylindraceum (6)

Prosopium williamsoni (5)

Salvelinus fontinalis $(2,8,11)$

Salvelinus namaycush $(1,4,5,7$, $9,10)$

Distribution: $\quad B C, M B, N F, N T, O N$, QC, SK

Records: 1) Stewart-Hay 1951c (MB);

2) Kooyman 1951 (MB); 3) McTavish 1952 (MB); 4) Stewart-Hay 1953b (MB);

5) Bangham \& Adams 1954 (BC); 6)

Bangham 1955 (ON); 7) Rawson 1959

(SK); 8) Hanek \& Molnar 1974 (QC); 9)

Stewart \& Bernier 1982 (NT); 10)

Anonymous 1984 (BC); 11) Marcogliese

\& Cone 1991 (NF)

Copepoda gen. sp.

Sites: buccal cavity, fins, gills

Hosts: Gasterosteus aculeatus (3)

Oncorhynchus mykiss (2)

Stizostedion vitreum (1)

Distribution: $\quad \mathrm{BC}, \mathrm{NF}, \mathrm{ON}$

Records: 1) Moore 1936 (ON); 2) Frost

1940 (NF); 3) Bangham \& Adams 1954

(BC)

CLASS ARACHNOIDEA

ORDER ACARINA

SUBORDER TROMBIDIFORMES

SUPERFAMILY HYDRACHNAE
FAMILY HYDRACHNIDAE

*Hydrachna sp.

Site: gills

Host: Perca flavescens

Distribution: ON

Record: Tedla \& Fernando 1969a

SUPERFAMILY PIONAE

FAMILY UNIONICOLIDAE

*Unionicola sp.

Site: viscera

Hosts: Coregonus clupeaformis $(2,3)$

Oncorhynchus mykiss (2)

Prosopium williamsoni $(1,2,3)$

Ptychocheilus oregonensis (1)

Distribution: $\quad \mathrm{BC}$

Records: 1) Anonymous $1981 ; 2$ ) Ching \& Parker 1983; 3) Anonymous 1984 
ORDER PETROMYZONTIFORMES

FAMILY PETROMYZONTIDAE lampreys

Lampetra japonica (Martens) Arctic lamprey

Cestoidea:

*Triaenophorus crassus (NT)

CLASS OSTEICHTHYES

ORDER ACIPENSERIFORMES

FAMILY ACIPENSERIDAE - sturgeons

Acipenser fulvescens Rafinesque, 1817

lake sturgeon

Cnidaria:

Polypodium hydriforme (MB, SK)

Polypodium sp. (MB, SK)

Monogenea:

Diclybothrium atriatum (MB, ON, QC, SK)

Diclybothrium hamulatum (ON) 
Paradiclybothrium sp. (ON)

Digenea:

Allocreadiidae gen. sp. (ON)

Allocreadium sp. (ON)

Azygia longa (MB)

Bunodera luciopercae (ON)

Crepidostomum auriculatum (MB, $\mathrm{ON}$, QC, SK)

Deropristis hispidus (QC)

Digenea gen. sp. (ON)

*Diplostomum spathaceum (ON)

*Diplostomum sp. (MB, ON, QC, SK)

Homalometron armatum (ON)

Skrjabinopsolus manteri (ON)

Cestoidea:

Cestoidea gen. sp. (ON)

*Proteocephalidae gen. sp. (MB, ON, SK)

*Tetraphyllidea gen. sp. (MB)

*Triaenophorus nodulosus (ON)

Nematoda:

**Nematoda gen. sp. (ON)

**Raphidascaris sp. (ON)

Rhabdochona cascadilla (MB, ON)

Rhabdochonidae gen. sp. (SK)

Spinitectus acipenseri (MB, SK)

Spinitectus gracilis (MB, ON, SK)

Spinitectus sp. (MB, ON, SK)

Sterliadochona sp. (MB, SK)

Truttaedacnitis clitellarius $(\mathrm{MB}, \mathrm{ON}$, SK)

Acanthocephala:

Acanthocephala gen. sp. (MB, ON, SK)
Echinorhynchus lateralis (ON)

Echinorhynchus leidyi (MB)

Leptorhynchoides thecatus (MB)

Neoechinorhynchus rutili (ON)

Neoechinorhynchus tenellus (MB)

Pomphorhynchus bulbocolli (MB, SK)

Annelida:

Glossiphonidae gen. sp. (MB)

Hirudinea gen. sp. (MB, SK)

Macrobdella decora (ON)

Piscicola punctata (ON)

Piscicola sy. (MB)

Placobdella montifera (MB)

Placobdella sp. (MB)

Mollusca:

*Unionidae gen. sp. (ON)

Branchiura:

Argulus stizostethi (ON)

ORDER OSTEOGLOSSIFORMES

FAMILY HIODONTIDAE - mooneyes

Hiodon alosoides (Rafinesque)

Digenea:

Azygia longa (MB)

Bucephalus sp. (MB, ON)

Crepidostomum illinoiense (MB)

Crepidostomum sp. (MB)

*Heterophyes sp. (MB)

Lissorchis crassicrurum (MB) 
Paurorhynchus hiodontos (AB, MB, SK)

Cestoidea:

Bothriocephalus cuspidatus (AB, HBD, MB, ON, SK)

${ }^{*}$ Ligula intestinalis (MB)

Nematoda:

**Raphidascaris acus (MB)

Rhabdochona canadensis (MB)

Copepoda:

Ergasilus nerkae (MB)

Ergasilus sp. (MB)

Hiodon tergisus Leseuer mooneye

Monogenea:

Mazocraeoides sp. (ON)

Digenea:

Crepidostomum illinoiense (MB, $\mathrm{ON})$

${ }^{*}$ Heterophyes sp. (MB)

Leuceruthrus micropteri (ON)

Paurorhynchus hiodontos (MB)

Plagioporus serratus (QC)

*Tetracotyle sp. (ON)

Cestoidea:

Bothriocephalus cuspidatus (HBD, ON)

Bothriocephalus sp. (AB, NT)

*Proteocephalus stizostethi $(\mathrm{ON})$

Proteocephalus sp. (ON)
Nematoda:

Camallanus oxycephalus (ON)

Rhabdochona cascadilla (ON)

Sterliadochona ephemeridarum (MB)

Acanthocephala:

Pomphorhynchus bulbocolli (MB, ON)

\section{ORDER CYPRINIFORMES}

FAMILY C'YPRINIDAE - minnows

Couesius plumbeus (Agassiz) lake chub

Monogenea:

Cleidodiscus brachus (LB, ON)

Dactylogyrus banghami (BC, ON)

Dactylogyrus mylocheilus $(\mathrm{BC}, \mathrm{ON})$

Gyrodactyloidea gen. sp. (BC)

Gyrodactylus aldrichi (LB)

Gyrodactylus couesius (BC, ON)

Gyrodactylus plumbeus (LB)

Octomacrum semotili (ON)

Octomacrum sp. (ON)

Digenea:

Allocreadium lobatum (BC, LB)

*Crassiphialia bulboglossa (ON)

*Diplostomum baeri bucculentum (BC)

*Diplostomum spathaceum (BC, ON, QC)

*Diplostomum sp. (BC, ON, QC)

*Neascus sp. (BC, ON)

Plagioporus cooperi (ON)

*Posthodiplostomum minimum (BC, 
ON)

*Tetracotyle sp. (BC, LB, ON)

Cestoidea:

Eubothrium sp. (BC)

*Hymenolepis sp. (BC)

*Ligula intestinalis (AB, BC)

Proteocephalus sp. (BC, ON)

Nematoda:

Nematoda gen. sp. (BC)

Pseudocapillaria (Pseudocapillaria) tomentosa (BC)

Rhabdochona canadensis (AB, $\mathrm{ON}$ )

Rhabdochona cascadilla (BC)

Rhabdochona sp. (ON)

Acanthocephala:

Echinorhynchus salmonis (ON)

Neoechinorhynchus rutili (BC, ON)

Mollusca:

*Unionidae gen. sp. (BC, ON)

Copepoda:

Ergasilus nerkae (BC)

Erguillus sp. (ON)

Hybognathus hankinsoni Hubbs brassy

minnow

Monogenea:

Dactylogyrus hankinsoni (ON)

Dactylogyrus sp. (ON)

Gyrodactylus sp. (ON)
Octomacrum sp. (ON)

Digenea:

*Clinostomum complanatum (ON)

*Diplostomum spathaceum (ON)

*Diplostomum sp. (ON)

*Neascus sp. (ON)

*Ornithodiplostomum ptychocheilus (ON)

Plagioporus sinitsini (ON)

*Posthodiplostomum minimum minimum (ON)

${ }^{*}$ Rhipidocotyle sp. (ON)

*Tetracotyle sp. (ON)

*Uvulifer ambloplitis (ON)

Cestoidea:

*Ligula intestinalis (ON)

Mollusca:

*Anodontoides ferussacinea (ON)

*Unionidae gen. sp. (ON)

Margariscus margarita Cope pearl dace

Monogenea:

Cleidodiscus brachus (NB, ON)

Dactylogyrus banghami (ON)

Dactylogyrus sp. (NB, ON)

Gyrodactylus margaritae (ON)

Octomacrum microconfibula (NB)

Octomacrum semotili (ON)

Octomacrum sp. (ON)

Digenea:

Crepidostomum sp. (ON) 
*Digenea gen. sp. (MB)

*Diplostomum sp. (QC)

*Neascus sp. (ON)

*Tetracotyle sp. (ON)

*Uvulifer ambloplitis (ON)

Cestoidea:

Proteocephalus pearsei (EXP)

Nematoda:

Rhabdochona canadensis (ON)

Rhabdochona cascadilla (ON, QC)

Annelida:

Piscicola sp. (ON)

Notropis atherinoides Rafinesque

emerald shiner

Monogenea:

Dactylogyrus sp. (ON)

Gyrodactyloidea gen. sp. (ON)

Gyrodactylus sp. (ON)

Digenea:

*Centrovarium lobotes (MB)

Digenea gen. sp. (ON)

*Diplostomum spathaceum (ON)

*Diplostomum spathaceum indistinctum (ON)

*Diplostomum sp. (MB, ON, SK)

Leuceruthrus micropteri (ON)

Plagioporus cooperi (ON)

*Posthodiplostomum cuticola (ON)

*Posthodiplostomum minimum minimum (ON)
*Strigeidae gen. sp. (ON)

Cestoidea:

Bothriocephalus cuspidatus (ON)

*Ligula intestinalis (SK)

*Proteocephalus ambloplitis (ON)

Proteocephalus pearsei (ON)

Proteocephalus pinguis (ON)

Proteocephalus sp. (ON)

Nematoda:

Camallanus oxycephalus (ON)

*Contracaecum sp. (MB)

Hysterothylacium brachyurum (ON)

Rhabdochona sp. (ON)

Acanthocephala:

Neoechinorhynchus sp. (ON)

Mollusca:

*Unionidae gen. sp. (ON)

Branchiura:

Argulus appendiculosus (MB)

Notropis hudsonius (Clinton) spottail shiner

Monogenea:

Dactylogyrus sp. (ON)

Gyrodactyloidea gen. sp. (ON)

Gyrodactylus sp. (ON)

Octomacrum semotili (ON)

Digenea: 
Allocreadium lobatum (ON)

*Apophallus brevis (MB, ON)

Bucephalus sp. (ON)

${ }^{*}$ Centrovarium lobotes $(\mathrm{ON})$

Centrovarium lobotes (ON)

*Clinostomum complanatum (MB, ON)

*Crassiphialia bulboglossa (MB, ON)

${ }^{*}$ Cryptogonimus chili (ON)

**Digenea gen. sp. (MB)

*Diplostomum spathaceum (MB, ON, SK)

*Diplostomum sp. (MB, ON, SK)

Leuceruthrus micropteri (ON)

Lissorchis sp. (ON)

*Neascus sp. (AB, ON)

Plagioporus cooperi (ON)

Plagioporus sinitsini (ON)

*Posthodiplostomum minimum (ON)

*Posthodiplostomum minimum minimum (ON)

Sanguinicola sp. (ON)

* Tetracotyle sp. (ON)

*Uvulifer ambloplitis (ON)

Cestoidea:

Bothriocephalus cuspidatus (MB, ON)

Glaridacris sp. (ON)

*Ligula intestinalis $(\mathrm{AB}, \mathrm{MB}, \mathrm{ON}$, SK)

*Ligula sp. (MB)

Proteocephalus ambloplitis (ON)

Proteocephalus pinguis (MB, ON)

Proteocephalus sp. (ON)

*Schistocephalus solidus (MB)

*Schistocephalus sp. (AB)

Spartoides wardi (MB, ON)

*Triaenophorus nodulosus (MB, ON)

Nematoda:
Camallamus oxycephalus (ON)

*Contracaecum sp. (LB, MB, ON)

*Cosmocephalus obvelatus (ON)

**Nematoda gen. sp. (ON)

Paracuaria adunca (ON)

*Raphidascaris acus (MB)

Rhabdochona cascadilla (ON)

Rhabdochona decaturensis (ON)

Rhabdochona sp. (MB)

Spinitectus gracilis $(\mathrm{ON})$

Acanthocephala:

Echinorhynchus salmonis (ON)

Leptorhynchoides thecatus (ON)

Neoechinorhynchus notemigoni (ON)

Neoechinorhynchus rutili (ON)

Neoechinorhynchus sp. (ON)

Pomphorhynchus bulbocolli (MB, ON)

Pomphorhynchus sp. (MB)

Annelida:

Myzobdella lugubris (MB)

Branchiura:

Argulus stizostethi (ON)

Copepoda:

Ergasilus sp. (AB, MB, ON)

Phoxinus eos Cope northern redbelly dace

Monogenea:

Cleidodiscus brachus (NB)

Dactylogyrus chrosomi (ON)

Dactylogyrus eos (NB, ON)

Gyrodactylidae gen. sp. (ON) 
Gyrodactyloidea gen. sp. (ON)

Gyrodactylus sp. (NB, ON)

Octomacrum microconfibula (NB)

Octomacrum semotili (ON)

Digenea:

Allocreadium isoporum (QC)

*Clinostomum complanatum (ON)

*Clinostomum sp. (QC)

*Crassiphialia bulboglossa (QC)

*Digenea gen. sp. (ON)

*Diplostomum sp. (ON)

*Neascus sp. (ON)

Phyllodistomum sp. (ON)

*Posthodiplostomum minimum (ON)

*Tetracotyle sp. (ON)

*Uvulifer ambloplitis (ON)

Cestoidea:

*Ligula intestinalis (ON)

*Paradilepis sp. (ON)

Nematoda:

*Contracaecum sp. (QC)

**Nematoda gen. sp. (ON)

Rhabdochona canadensis (ON)

Rhabdochona cascadilla (ON, QC)

*Tetrameres sp. (QC)

Acanthocephala:

Neoechinorhynchus rutili (ON)

Pomphorhynchus bulbocolli (ON, QC)

Mollusca:

*Anodontoides ferrussacinea (ON)

*Unionidae gen. sp. (ON)
Phoximus neogaeus finescale dace

Monogenea:

Cleidodiscus brachus (ON)

Dactylogyrus chrosomi (ON)

Dactylogyrus sp. (ON)

Gyrodactylidae gen. sp. (ON)

Gyrodactylus sp. (ON)

Octomacrum semotili (ON)

Digenea:

*Clinostomum complanatum (ON)

Diplostomum spathaceum (ON)

Ichthyocotylurus platycephalus $(\mathrm{ON})$

*Neascus sp. (ON)

Phyllodistomum sp. (ON)

*Posthodiplostomum minimum (ON)

*Tetracotyle sp. (ON)

*Uvulifer ambloplitis (ON)

Cestoidea:

*Paradilepis sp. (ON)

Pliovitellaria wisconsinensis (ON)

Proteocephalus sp. (ON)

Nematoda:

**Nematoda gen. sp. (ON)

Rhabdochona canadensis (ON)

*Spiroxys sp. (ON)

Acanthocephala:

Neoechinorhynchus rutili (ON)

Mollusca:

*Unionidae gen. sp. (ON) 
Branchiura:

Argulus catostomi (ON)

Pimephales promelas Rafinesque

fathead minnow

Monogenea:

Dactylogyrus bifurcatus (ON)

Dactylogyrus bychowskyi (ON)

Dactylogyrus mollis (ON)

Dactylogyrus sp. (ON)

Gyrodactyloidea gen. sp. (ON)

Gyrodactylus hoffmani (ON)

Gyrodactylus lacustris (ON)

Gyrodactylus sp. (ON)

Octomacrum lanceatum (ON)

Octomacrum semotili (ON)

Digenea:

Allocreadium lobatum (ON)

*Clinostomum complanatum (ON)

*Crassiphialia bulboglossa (MB, ON)

*Diplostomum spathaceum (ON)

*Diplostomum sp. (ON)

*Neascus sp. (ON)

*Ornithodiplostomum ptychocheilus (ON)

Plagioporus sinitsini (ON)

*Posthodiplostomum minimum (ON)

*Posthodiplostomum minimum minimum (ON)

Sanguinicola sp. (ON)

*Uvulifer ambloplitis (ON)

Cestoidea:

Biacetabulum sp. (ON)

${ }^{*}$ Ligula intestinalis $(\mathrm{ON}, \mathrm{SK})$
Pliovitellaria wisconsinensis (ON)

**Proteocephalus sp. (ON)

Nematoda:

Contracaecum sp. (AB, MB)

${ }^{*}$ Raphidascaris acus (ON)

Rhabdochona sp. (ON)

Acanthocephala:

Neoechinorhynchus sp. (ON)

Mollusca:

* Lasmigona compressa (ON)

*Unionidae gen. sp. (ON)

Copepoda

Ergasilus cyprinaceus (ON):

Platygobio gracilis (Richardson)

flathead chub

Monogenea:

Cleidodiscus brachus (AB)

Dactylogyrus albertensis (AB)

Nematoda:

Rhabdochona canadensis (AB)

Ptychocheilus oregonensis (Richardson) northern squawfish

Monogenea:

Dactylogyrus columbiensis (BC) 
Dactylogyrus ptychocheilus (BC)

Dactylogyrus tridactylus (BC)

Dactylogyrus vancleavei (BC)

Gyrodactyloidea gen. sp. (BC)

Gyrodactylus sp. (BC)

Digenea:

Allocreadium isoporum (BC)

Allocreadium lobatum (BC)

Crepidostomum farionis (BC)

*Diplostomum baeri bucculentum (BC)

*Diplostomum spathaceum $(\mathrm{BC})$

*Diplostomum sp. (BC)

*Ichthyocotylurus platycephalus (BC)

*Neascus sp. (BC)

Phyllodistomum sp. (BC)

*Posthodiplostomum minimum (BC)

*Strigeidae gen. sp. (BC)

*Tetracotyle sp. (BC)

*Tylodelphys scheuringi (BC)

Cestoidea:

Bothriocephalus acheilognathi (BC)

Cestoidea gen. sp. (BC)

Eubothrium salvelini $(\mathrm{AB}, \mathrm{BC})$

Eubothrium tulipai (BC)

Eubothrium sp. (BC)

${ }^{*}$ Ligula intestinalis (BC)

*Paradilepis simoni (BC)

Proteocephalus ptychocheilus (BC)

Nematoda:

Capillariidae gen. sp. (BC)

*Contracaecum spiculigerum (BC)

*Eustrongylides sp. (BC)

**Hysterothylacium sp. (BC)

Pseudocapillaria (Pseudocapillaria) tomentosa (BC)
*Raphidascaris acus (BC)

Rhabdochona cascadilla (BC)

Rhabdochona sp. (BC)

*Spiroxys sp. (BC)

Truttaedacnitis truttae (BC)

Acanthocephala:

Neoechinorhynchus rutili (BC)

Neoechinorhynchus saginatus (BC)

Neoechinorhynchus salmonis (BC)

Neoechinorhynchus venustus (BC)

Pomphorhynchus bulbocolli (BC)

Annelida:

Illinobdella sp. (BC)

Myzobdella sp. (BC)

Piscicola punctata (BC)

Mollusca:

*Anodonta sp. (BC)

*Unionidae gen. sp. (BC)

Copepoda:

Ergasilus nerkae (BC)

Arachnoidea:

*Unionicola sp. (BC)

Rhinichthys cataractae (Valenciennes) longnose dace

Monogenea:

Dactylogyrus banghami (AB, BC, ON)

Gyrodactyloidea gen. sp. (BC) 
Gyrodactylus atratuli (ON)

Gyrodactylus dechtiari (ON)

Gyrodactylus stunkardi (LB, ON)

Digenea:

Allocreadium lobatum (ON)

*Crassiphialia bulboglossa (ON)

*Digenea gen. sp. (MB)

*Diplostomum spathaceum (ON)

*Diplostomum sp. (MB, ON, SK)

*Neascus rhinichthysi (ON)

*Neascus sp. (BC, ON)

Plagioporus cooperi (ON)

*Posthodiplostomum minimum (BC, ON)

*Tetracotyle sp. (BC, ON)

*Tylodelphys scheuringi (ON)

Cestoidea:

Cestoidea gen. sp. (AB)

*Ligula sp. (AB)

*Triaenophorus nodulosus (ON)

Nematoda:

Camallanus oxycephalus (ON)

Rhabdochona canadensis (AB, $\mathrm{ON}$ )

Rhabdochona cascadilla (BC)

Rhabdochona milleri (BC)

*Spiroxys sp. (ON)

Sterliadochona ephemeridarum (BC)

**Truttaedacnitis sp. (BC)

Acanthocephala:

Pomphorhynchus bulbocolli (ON)

Copepoda:

Ergasilus caeruleus $(\mathrm{ON})$
Richardsonius balteatus (Richardson) redside shiner

Monogenea:

Dactylogyrus banghami (BC)

Dactylogyrus richardsonius (BC)

Gyrodactyloidea gen. sp. (BC)

Octomacrum microconfibula (BC)

Octomacrum sp. (BC)

Pellucidhaptor pellucidhaptor (BC)

Digenea:

Allocreadium lobatum (BC)

${ }^{*}$ Clinostomum complanatum (BC)

*Diplostomum sp. (BC)

${ }^{*}$ Neascus sp. (BC)

Phyllodistomum sp. (BC)

*Posthodiplostomum minimum (BC)

*Tetracotyle sp. (BC)

*Tylodelphys scheuringi (BC)

Cestoidea:

Eubothrium sp. (BC)

${ }^{*}$ Ligula intestinalis (BC)

${ }^{*}$ Ligula sp. (AB, MB)

Proteocephalus sp. (BC)

*Schistocephalus sp. (BC)

Nematoda:

Pseudocapillaria (Pseudocapillaria) tomentosa (BC)

Rhabdochona cascadilla (BC)

Rhabdochona sp. (BC)

${ }^{*}$ Spiroxys sp. (BC)

Truttaedacnitis sp. (BC)

Acanthocephala: 
Neoechinorhynchus cylindratus (BC)

Neoechinorhynchus rutili (BC)

Neoechinorhynchus sp. (BC)

Pomphorhynchus bulbocolli (BC)

Mollusca:

*Unionidae gen. sp. (BC)

Copepoda:

Ergasilus nerkae (BC)

FAMILY CATOSTOMIDAE - suckers

Carpiodes cyprinus (Lesueur) quillback

Monogenea:

Acolpenteron catostomi (ON)

Anonchohaptor anomalum (MB, ON)

Anonchohaptor muelleri (ON)

Anonchohaptor sp. (ON)

Gyrodactylus sp. (ON)

Icelanonchohaptor fyviei (ON)

Icelanonchohaptor microcotyle $(\mathrm{CN})$

Neodiscocotyle carpioditis (ON)

Pellucidhaptor angularis (ON)

Pellucidhaptor eremitus (ON)

Pellucidhaptor eriensis (ON)

Pellucidhaptor micracanthus (ON)

Pellucidhaptor sp. (ON)

Digenea:

Digenea gen. sp. (MB)

*Diplostomum spathaceum (ON)

*Diplostomum sp. (MB, ON, SK)

Lissorchis attenuatus (ON)

Lissorchis gullaris (MB)
Phyllodistomum lysteri (ON)

*Posthodiplostomum minimum (ON)

*Posthodiplostomum minimum minimum (ON)

Sanguinicola sp. (ON)

*Tetracotyle sp. (ON)

Cestoidea:

Biacetabulum sp. (ON)

Glaridacris confusus (ON)

Glaridacris terebrans (ON)

Glaridacris sp. (AB, BC)

Hunterella nodulosa $(\mathrm{AB}, \mathrm{ON})$

Hypocaryophyllaeus paratarius (ON)

Isoglaridacris calentinei (BC)

*Ligula intestinalis (MB)

Monobothrium hunteri (MB, ON)

Monobothrium sp. (BC)

Proteocephalus sp. (LB, ON)

Rowardleus pennensis (MB)

Spartoides wardi (MB, ON)

*Triaenophorus nodulosus (ON)

Nematoda:

Camallanus ancylodirus (ON)

Camallanus oxycephalus (ON)

Philometroides nodulosa (ON)

Rhabdochona cascadilla (ON)

Rhabdochona milleri (ON)

Acanthocephala:

Neoechinorhynchus carpiodi (MB, $\mathrm{ON}$ )

Neoechinorhynchus crassus (ON)

Neoechinorhynchus sp. (MB)

Pomphorhynchus bulbocolli (MB, ON)

Mollusca: 
*Unionidae gen. sp. (MB, ON)

Branchiura:

Argulus appendiculosus (MB)

Argulus catostomi (ON)

Copepoda:

Ergasilus caeruleus (ON)

Ergasilus lizae (MB)

Ergasilus sp. (ON)

Catostomus catostomus (Forster) longnose sucker

Monogenea:

Acolpenteron catostomi (BC, ON, QC) Anonchohaptor anomalum (BC, LB, ON, YT)

Gyrodactyloidea gen. sp. (BC)

Gyrodactylus aquilinus (BC)

Gyrodactylus atratuli (LB)

Gyrodactylus spathulatus (LB)

Gyrodactylus sp. (ON)

Monogenea gen. sp. (BC)

Octomacrum lanceatum (BC, $\mathrm{ON})$

Pellucidhaptor catostomi (ON)

Pellucidhaptor nasalis (BC)

Pseu:'omurraytrema copulatum (BC, $\mathrm{ON})$

Digenea:

Allocreadium lobatum (BC, QC, SK)

Bucephalus sp. (BC, ON)

Crepidostomum farionis (BC)

*Digenea gen. sp. (MB)

*Diplostomum spathaceum (CA, LB, ON, YT)
*Diplostomum spathaceum

indistinctum (ON)

*Diplostomum sp. (BC, LB, QC)

*Ichthyocotylurus erraticus (ON)

*Ichthyocotylurus platycephalus (BC, ON)

Lissorchis attemuatus (BC, $\mathrm{ON})$

Lissorchis gullaris (LB)

Lissorchis simeri $(\mathrm{ON})$

Lissorchis sp. (ON)

*Metorchis conjunctus (QC, SK)

${ }^{*}$ Neascus sp. (AB, BC)

Phyllodistomum lysteri (ON)

Phyllodistomum sp. (ON)

Plagiocirrus sp. (BC)

Plagioporus sinitsini (ON)

*Posthodiplostomum minimum (BC, ON)

Sanguinicola sp. (ON)

Sanguinicolidae gen. sp. (BC)

*Tetracotyle sp. (BC, ON)

Cestoidea:

Caryophyllidea gen. sp. (AB, BC, ON)

Cestoidea gen. sp. (AB, BC, NT)

Eubothrium sp. (BC, MB)

Glaridacris catostomi (AB, BC, MB, NT, ON, QC, SK, YT)

Glaridacris terebrans $(\mathrm{BC})$

Glaridacris sp. (AB)

Hunterella nodulosa ( $\mathrm{AB}, \mathrm{LB}, \mathrm{ON})$

Isoglaridacris calentinei (BC)

${ }^{\star}$ Ligula intestinalis $(\mathrm{AB}, \mathrm{BC}, \mathrm{MB}$, ON, SK)

${ }^{*}$ Ligula sp. (AB)

Monobothrium sp. (BC)

Proteocephalus sp. (LB, ON)

*Schistocephalus sp. (BC)

*Triaenophorus nodulosus (ON)

*Triaenophorus sp. (ON) 
Nematoda:

Cystidicola farionis (YT)

*Eustrongylides tubifex (ON)

**Philometra sp. (QC)

Philometroides huronensis (BC, $\mathrm{ON}$ )

Philometroides nodulosus (ON)

Pseudocapillaria (Pseudocapillaria) tomentosa (BC, ON)

Raphidascaris acus (ON, QC)

Rhabdochona cascadilla $(\mathrm{AB}, \mathrm{BC}$, QC)

Rhabdochona catostomi (AB)

Rhabdochona ovifilamenta (AB)

Rhabdochona sp. (BC, LE)

Acanthocephala:

Acanthocephalus dirus (ON)

Echinorhynchus lateralis (QC)

Echinorhynchus leidyi (ON)

Echinorhynchus salmonis (AB, MB, ON)

Leptorhynchoides thecatus (ON)

Neoechinorhynchus crassus (LB, MB, ON)

Neoechinorhynchus rutili $(\mathrm{AB}, \mathrm{BC})$

Neoechinorhynchus saginatus (BC)

Neoechinorhynchus salmonis (BC)

Neoechinorhynchus strigosus (LB, $\mathrm{ON}$ )

Neoechinorhynchus venustus $(\mathrm{AB}$, $\mathrm{BC}, \mathrm{LB}, \mathrm{ON}, \mathrm{QC}$ )

Neoechinorhynchus sp. (NT)

Octospinifer macilentus (AB, BC, ON)

Pomphorhynchus bulbocolli (BC, ON)

Annelida:

Actinobdella inequiannulata (BC, ON, SK)
Actinobdella sp. (ON)

Myzobdella lugubris (SK)

Piscicola geometra (SK)

Mollusca:

*Unionidae gen. sp. (ON)

Branchiura:

Argulus catostomi (ON)

Copepoda:

Ergasilus caeruleus (LB, ON)

Ergasilus centrarchidarum (ON, QC)

Ergasilus nerkae (BC, ON)

Ergasilus sp. (AB)

Lernaea catostomi (SK)

Catostomus commersoni (Lacépède) white sucker

Monogenea:

Acolpenteron catostomi (BC, NB, ON) Anonchohaptor anomalum (BC, LB, $\mathrm{NB}, \mathrm{ON}, \mathrm{QC})$

Dactylogyrus sp. (ON)

Gyrodactyloidea gen. sp. (ON)

Gyrodactylidae gen. sp. (ON)

Gyrodactylus aquilinus (ON)

Gyrodactylus atratuli $(\mathrm{ON})$

Gyrodactylus commersoni (LB)

Gyrodactylus limi (ON)

Gyrodactylus spathulatus (ON)

Gyrodactylus stunkardi (ON)

Gyrodactylus sp. (ON)

Ligictaluridus pricei $(\mathrm{ON})$

Octomacrum lanceatum $(\mathrm{ON})$

Pellucidhaptor nasalis (BC, NB, ON) 
Pseudomurraytrema alabarrum (ON)

Pseudomurraytrema copulatum (BC, ON)

Pseudomurraytrema muelleri (AB)

Digenea:

Allocreadium lobatum (ON)

*Amphimerus elongatus (QC)

*Amphimerus pseudofelineus (MB)

*Apophallus venustus (QC)

Azygia angusticauda (ON)

Bucephalus sp. (ON)

*Clinostomum complanatum (ON, QC)

Crepidostomum cooperi (ON, QC)

Crepidostomum farionis (QC)

Crepidostomum sp. (QC)

Creptotrema funduli (ON)

**Digenea gen. sp. (MB)

*Diplostomum spathaceum (LB, ON)

*Diplostomum spathaceum indistinctum $(\mathrm{AB}, \mathrm{ON})$

*Diplostomum sp. (BC, MB, QC, SK)

*Ichthyocotylurus erraticus (ON)

*Ichthyocotylurus platycephalus (ON)

Lissorchis attenuatus ( $\mathrm{AB}, \mathrm{ON}, \mathrm{QC})$

Lissorchis crassicrurum (MB)

Lissorchis simeri (ON)

*Metorchis conjunctus (MB, ON, QC, SK)

*Neascus sp. (MB, ON)

*Ornithodiplostomum ptychocheilus (ON)

*Parametorchis manitobensis (MB)

Phyllodistomum lysteri (ON, QC)

Phyllodistomum superbum (QC)

Plagioporus serotinus (QC)

Plagioporus sinitsini (ON, QC)

*Posthodiplostomum minimum (ON)

*Posthodiplostomum minimum minimum (ON)
Sanguinicola sp. (ON)

*Tetracotyle sp. (BC, MB, ON)

*Uvulifer ambloplitis (ON)

Cestoidea:

Biacetabulum biloculoides (ON)

Biacetabulum sp. (MB, ON)

Caryophyllidea gen. sp. (AB)

*Diphyllobothrium sp. (QC)

Glaridacris catostomi (AB, BC, LB, $\mathrm{MB}, \mathrm{NB}, \mathrm{NT}, \mathrm{ON}, \mathrm{QC})$

Glaridacris confusus (ON, QC)

Glaridacris laruei (ON, QC)

Glaridacris sp. (AB, MB)

Hunterella nodulosa (AB, BC, LB, $\mathrm{MB}, \mathrm{ON}$ )

Hunterella sp. (AB)

Isoglaridacris bulbocirrus (ON)

${ }^{*}$ Ligula intestinalis (AB, MB, ON, QC, SK)

${ }^{*}$ Ligula sp. (AB, MB, QC)

Monobothrium hunteri (BC, ON)

Monobothrium sp. (BC)

*Triaenophorus nodulosus (ON)

*Triaenophorus sp. (ON)

Nematoda:

Camallamus oxycephalus (ON)

Capillaria (Capillaroides) catenta (QC)

Contracaecum sp. (LB, MB, ON, QC)

*Eustrongylides tubifex (ON)

*Eustrongylides sp. (BC, ON)

**Nematoda gen. sp. (ON, QC)

Philometra kobuleji (ON)

**Philometra sp. (ON)

Philometroides huronensis (BC, EXP, ON, QC)

Philometroides nodulosa $(\mathrm{MB}, \mathrm{ON}$, $\mathrm{QC)}$ 
Philometroides sp. (ON)

Pseudocapillaria (Pseudocapillaria) tomentosa (ON)

* Raphidascaris acus (MB, ON, QC)

Rhabdochona canadensis (MB)

Rhabdochona cascadilla ( $\mathrm{AB}, \mathrm{ON})$

Rhabdochona ovifilamenta (AB, ON, QC)

Rhabdochona sp. (LB, ON)

*Spiroxys sp. (ON)

Acanthocephala:

Acanthocephala gen. sp. (MB)

Acanthocephalus dirus (ON)

Echinorhynchus lateralis (QC)

Echinorhynchus leidyi (ON)

Echinorhynchus salmonis (AB, MB, ON)

Leptorhynchoides thecatus (ON)

Neoechinorhynchus crassus (MB, ON, SK)

Neoechinorhynchus cylindratus (QC)

Neoechinorhynchus distractus (MB)

Neoechinorhynchus rutili (AB)

Neoechinorhynchus saginatus (ON)

Neoechinorhynchus salmonis (BC)

Neoechinorhynchus strigosus (AB, LB, ON)

Neoechinorhynchus venustus $(A B$, $\mathrm{BC}, \mathrm{MB}, \mathrm{ON}$ )

Neoechinrohynchus sp. (AB, MB, ON)

Octospinifer macilentus $(\mathrm{AB}, \mathrm{BC}$, ON, QC)

Pomphorhynchus bulbocolli (AB, $\mathrm{BC}, \mathrm{MB}, \mathrm{ON}, \mathrm{QC})$

Pomphorhynchus sp. (MB)

Tanaorhamphus sp. (MB)

Annelida:
Actinobdella inequiannulata (EXP,

ON, QC, SK)

Cystobranchus meyeri (ON)

Myzobdella lugubris (MB)

Myzobdella sp. (ON)

Piscicola geometra (SK)

Piscicola milneri (AB)

Placobdella montifera (MB)

Mollusca:

* Anodonta cataracta cataracta (NS)

* Anodonta cataracta fragilis (NS)

* Anodonta implicata (NS)

*Anodonta plana (ON)

*Anodontoides ferrussacinea (ON)

* Elliptio complanata (NS)

* Lampsilis radiata radiata (NS)

*Lampsilis sp. (ON)

*Unionidae gen. sp. (MB, ON)

Branchiura:

Argulus appendiculosus (MB, ON) Argulus biramosus (AB)

Argulus catostomi (ON)

Argulus stizostethi (MB)

Copepoda:

Ergasilus caeruleus (LB, ON)

Ergasilus nerkae (BC)

Ergasilus versicolor $(\mathrm{MB})$

Ergasilus sp. (AB, $\mathrm{ON}$ )

Lernaea catostomi (SK)

Lernaea cyprinacea (MB, ON)

Catostomus macrocheilus Girard largescale sucker

Monogenea: 
Acolpenteron catostomi (BC)

Anonchohaptor anomalum (BC)

Gyrodactyloidea gen. sp. (BC)

Octomacrum lanceatum (BC)

Pellucidhaptor nasalis (BC)

Pseudomurraytrema copulatum (BC)

Digenea:

*Diplostomum sp. (BC)

*Ichthyocotylurus platycephalus (BC)

Lissorchis attenuatus $(\mathrm{AB}, \mathrm{BC})$

${ }^{*}$ Neascus sp. (BC)

*Posthodiplostomum minimum (BC)

*Tetracotyle sp. (BC)

*Tylodelphys scheuringi (BC)

Cestoidea:

Caryophyllidea gen. sp. (BC)

Glaridacris catostomi ( $\mathrm{AB}, \mathrm{BC})$

Glaridacris terebrans (BC)

Glaridacris vogei (BC)

Glaridacris sp. (BC)

Hunterella nodulosa (BC)

*Ligula intestinalis (BC)

Nematoda:

*Eustrongylides sp. (BC)

Philometroides huronensis (BC)

Pseudocapillaria (Pseudocapillaria)

tomentosa (BC)

Rhabdochona cascadilla (BC)

Rhabdochona zacconis (BC)

Rhabdochona sp. (BC)

*Spiroxys sp. (BC)

Acanthocephala:

Neoechinorhynchus crassus (BC)

Neoechinorhynchus rutili (BC)
Neoechinorhynchus saginatus (BC)

Neoechinorhynchus salmonis (BC)

Neoechinorhynchus venustus (BC)

Octospinifer macilentus (BC)

Pomphorhynchus bulbocolli (BC)

Annelida:

Actinobdella inequiannulata (BC)

Hirudinea gen. sp. (BC)

Piscicola salmositica (BC)

Piscicola sp. (BC)

Rhynchobdellidae gen. sp. (BC)

Copepoda:

Ergasilus nerkae (BC)

Catostomus platyrhynchus (Cope) mountain sucker

Nematoda:

Rhabdochona ovifilamenta (AB) Rhabdochona zacconis (BC)

Rhabdochona sp. (AB)

Catostomus sp.

Cestoidea:

Glaridacris catostomi (AB)

Moxostoma anisurum (Rafinesque) silver redhorse

Monogenea:

Anonchohaptor anomalum (ON)

Dactylogyrus urus (ON) 
Gyrodactylus spathulatus (ON)

Gyrodactylus sp. (ON)

Pellucidhaptor moxostomi (ON)

Pellucidhaptor thelostea (ON)

Pseudomurraytrema copulatum (ON)

Pseudomurraytrema sp. (ON)

Digenea:

*Diplostomum spathaceum indistinctum (ON)

*Diplostomum sp. (MB, SK)

Lissorchis attenuatus (ON)

Lissorchis crassicrurum (MB)

*Neascus sp. (MB, ON)

Sanguinicola sp. (ON)

*Tetracotyle sp. (MB)

Cestoidea:

Biacetabulum infrequens (ON)

Biacetabulum sp. (MB)

Cestoidea gen. sp. (MB)

Khawia iowensis (MB)

*Triaenophorus nodulosus (ON)

Nematoda:

Contracaecum sp. (MB)

**Hysterothylacium brachyurum (ON)

**Nematoda gen. sp. (MB, ON)

*Raphidascaris acus (MB)

Rhabdochona canadensis (MB)

Spinitectus gracilis (ON)

Acanthocephala:

Leptorhynchoides thecatus (ON)

Neoechinorhynchus crassus (MB)

Neoechinorhynchus distractus (MB)

Neoechinorhynchus venustus (MB)

Pomphorhynchus bulbocolli (MB, ON)
Annelida:

Cystobranchus verrilli (MB)

Myzobdella lugubris (MB)

Placobdella montifera (MB)

Mollusca:

*Unionidae gen. sp. (MB)

Copepoda:

Ergasilus caeruleus (ON)

Ergasilus versicolor (MB)

Moxostoma macrolepidotum (Leseuer)

shorthead redhorse

Monogenea:

Anonchohaptor anomalum (ON)

Dactylogyrus duquesni (ON)

Dactylogyrus urus $(\mathrm{ON})$

Dactylogyrus sp. (ON)

Gyrodactylus sp. (ON)

Pellucidhaptor eriensis (ON)

Pellucidhaptor thelostea (ON)

Pellucidhaptor sp. (ON)

Pseudomurraytrema copulatum (ON)

Pseudomurraytrema sp. (ON)

Digenea:

*Apophallus venustus (QC)

*Diplostomum spathaceum

indistinctum (ON)

${ }^{*}$ Neascus sp. (ON)

Phyllodistomum lysteri (ON)

Phyllodistomum sp. (ON)

Plagioporus serotinus (QC)

Sanguinicola sp. (ON) 
Cestoidea:

Glaridacris catostomi (ON)

*Triaenophorus nodulosus (ON)

Nematoda:

Philometroides huronensis (ON) Rhabdochona cascadilla (BC, $\mathrm{ON})$

Rhabdochona milleri (ON, QC)

Neoechinorhynchus crassus (MB, ON)

Pomphorhynchus bulbocolii (MB, ON)

Annelida:

Cystobranchus verrilli (MB)

Myzobdella lugubris (MB)

Placobdella montifera (MB)

Branchiura:

Argulus catostomi (ON)

Copepoda:

Ergasilus caeruleus $(\mathrm{ON})$

Lernaea catostomi (SK)

ORDER SILURIFORMES

FAMILY ICTALURIDAE -

bullhead catfishes
Acanthocephala:

Ligictaluridus posthon (ON)

Ligictaluridus pricei $(\mathrm{ON})$

Digenea:

Acetodextra amiuri (ON)

Allocreadiidae gen. sp. (ON)

Alloglossidium corti (ON)

Alloglossidium geminus $(\mathrm{ON})$

Megalonia ictaluri (ON)

Cestoidea:

Corallobothrium fimbriatum (ON)

*Proteocepinalus xmbloplitis (ON)

Proteocephalus sp. (ON)

Nematoda:

Camallanus oxycephalus (ON)

*Hysterothylacium brachyurum (ON)

Rhabdochona canadensis (AB)

Rhabdochona decaturensis (ON)

Spinitectus gracilis $(\mathrm{ON})$

Acanthocephala:

Leptorhynchoides thecatus (ON)

Copepoda:

Ergasilus versicolor (ON)

ORDER SALMONIFORMES

FAMILY ESOCIDAE - pikes

Noturus flavus Rafinesque stonecat

Monogenea: northern pike 
Monogenea:

Dactylogyridae gen. sp. (AB)

Gyrodactyloidea gen. sp. (ON)

Gyrodactylus lucii (ON)

Gyrodactylus sp. (ON)

Tetraonchus loftusi (ON)

Tetraonchus monenteron (AB, LB, $\mathrm{MB}, \mathrm{ON}, \mathrm{YT}$ )

Digenea:

Allocreadium isoporum (YT)

Allocreadium lobatum (YT)

*Apophallus venustus (QC)

Azygia angusticauda (ON)

Azygia longa (MB, ON, QC)

Bunodera luciopercae (LB)

*Centrovarium lobotes (MB, ON)

Centrovarium lobotes (MB, ON, QC)

*Clinostomum complanatum (ON, QC)

*Crassiphialia bulboglossa (QC)

Crepidostomum cooperi (LB)

Crepidostomum farionis (MB)

Crepidostomum metoecus (YT)

*Digenea gen. sp. (AB, MB, QC)

Digenea gen. sp. (AB, MB)

*Diplostomum spathaceum (ON, NT)

*Diplostomum sp. (AB, MB, ON, QC, SK)

*Microphallus opacus (ON, QC)

*Neascus sp. (MB)

*Opisthorchis sp. (ON)

Phyllodistomum folium (QC)

Phyllodistomum superbum (QC)

Phyllodistomum sp. (ON)

*Posthodiplostomum minimum (ON)

* Tetracotyle parvula (QC)

* Tetracotyle sp. (MB, ON)

*Uvulifer ambloplitis (ON)
Cestoidea:

Bothriocephalus cuspidatus (AB, HBD, MB, ON)

Bothriocephalus sp. (MB)

Cestoidea gen. sp. (AB, MB, QC)

Cyathocephalus truncatus (MB)

*Diphyllobothrium latum (AB, MB,

NT, ON, QC, SK)

*Diphyllobothrium sp. (AB, MB, NT)

Eubothrium sp. (AB, MB)

Glaridacris catostomi (MB)

*Ligula sp. (MB)

Proteocephalus longicollis (YT)

Proteocephalus pinguis $(\mathrm{AB}, \mathrm{BC}, \mathrm{LB}$,

$\mathrm{MB}, \mathrm{NT}, \mathrm{ON}, \mathrm{QC})$

Proteocephalus stizostethi (ON, QC)

Proteocephalus sp. (AB, LB, MB,

QC)

*Schistocephalus solidus (AB)

*Triaenophorus crassus (AB)

Triaenophorus crassus (AB, BC, HBD, LB, MB, NT, ON, QC, SK, YT)

*Triaenophorus nodulosus (MB)

Triaenophorus nodulosus (AB, CA, $\mathrm{MB}, \mathrm{NT}, \mathrm{ON}, \mathrm{SK}, \mathrm{YT}$ )

*Triaenophorus sp. (HBD)

Triaenophorus sp. (AB, MB, NT)

Nematoda:

Camallanus oxycephalus (ON)

Camallanus sp. (LB)

*Contracaecum sp. (MB)

Cystidicola sp. (AB)

Hysterothylacium brachyurum (MB, $\mathrm{ON}, \mathrm{QC})$

**Nematoda gen. sp. (AB, MB)

**Philonema sp. (NT)

*Raphidascaris acus (MB, ON, YT)

Raphidascaris acus (AB, EXP, LB, 
$\mathrm{MB}, \mathrm{NT}, \mathrm{ON}, \mathrm{QC}, \mathrm{SK}, \mathrm{YT}$ )

** Raphidascaris sp. (AB, LB, MB)

Rhabdochona canadensis (AB, LB,

QC)

Spinitectus carolini (ON)

Spinitectus gracilis (MB, ON)

Acanthocephala:

Acanthocephala gen. sp. (MB)

Acanthocephalus dirus (ON)

Acanthocephalus sp. (MB)

Echinorhynchus lateralis (LB, ON)

Echinorhynchus leidyi (MB, NT)

Echinorhynchus sclmonis (AB, MB, NT, ON)

Leptorhynchoides thecatus (ON, QC)

Neoechinorhynchus cylindratus (ON)

Neoechinorhynchus rutili $(\mathrm{BC}, \mathrm{ON}$, YT)

Neoechinorhynchus strigosus (AB)

Neoechinorhyhchus tenellus (ON)

Neoechinorhynchus tumidus (YT)

Neoechinorhynchus sp. (ON)

Pomphorhynchus bulbocolli (MB, ON)

Annelida:

Desserobdella phalera (EXP, ON)

Illinobdella sp. (ON)

Mollibdella grandis (ON)

Myzcidella sp. (ON)

Piscicola geometra (SK)

Piscicola milneri (MB, NT, YT)

Placobdella montifera (MB)

Mollusca:

*Unionidae gen. sp. (MB, ON)

Branchiura:
Argulus appendiculosus (MB)

Argulus stizostethi (MB)

Argulus sp. (ON)

Copepoda:

Ergasilus caeruleus (ON)

Ergasilus luciopercarum (MB)

Salmincola extensus (MB)

FAMILY SALMONIDAE - trouts

Coregonus artedii Lesueur cisco or lake herring

Monogenea:

Discocotyle sagittata $(\mathrm{AB}, \mathrm{MB}, \mathrm{ON})$

Digenea:

Crepidostomum cooperi (CA, ON)

Crepidostomum farionis (AB, CA, $\mathrm{MB}, \mathrm{ON}$ )

Digenea gen. sp. (AB)

*Diplostomum spathaceum (CA, MB, ON)

*Diplostomum spathaceum indistinctum (MB, ON)

*Diplostomum sp. (ON)

*Ichthyocotylurus erraticus (AB, MB, ON)

*Neascus sp. (MB)

Phyllodistomum sp. (ON)

*Tetracotyle sp. (MB, ON)

Cestoidea:

Bothrimonas sturionis (MB)

Bothriocephalidae gen. sp. (ON) 
${ }^{*}$ Cestoidea gen. sp. (ON)

Cestoidea gen. sp. (MB)

Cyathocephalus truncatus $(\mathrm{AB}, \mathrm{MB}$, ON)

*Diphyllobothrium dendriticum (MB, $\mathrm{ON}, \mathrm{QC})$

*Diphyllobothrium ditremum (ON)

*Diphyllobothrium laruei (ON)

*Diphyllobothrium sp. (AB, MB, NT, ON)

Eubothrium crassum (NT, ON)

Eubothrium salvelini $(\mathrm{AB}, \mathrm{ON})$

Eubothrium sp. (YT)

Proteocephalus coregoni (MB, ON)

Proteocephalus filicollis (MIB, NT,

ON)

Proteocephalus longicollis (MB, NT, ON)

Proteocephalus pearsei (ON)

Proteocephalus sp. (AB, BC, LB, MB, NT, ON, QC)

*Triaenophorus crassus (AB, BC, CA, EXP, MB, NT, ON, QC, SK)

Triaenophorus nodulosus (AB, MB)

*Triaenophorus sp. (AB, MB, QC, SK)

Nematoda:

Cystidicola farionis (AB, MB, ON)

Cystidicola serrata (ON)

Cystidicola sp. (AB, ON)

Hysterothylacium brachyurum (MB)

**Nematoda gen. sp. (MB)

**Philometra sp. (ON)

Philonema agubernaculum (AB, BC)

**Philonema oncorhynchi (BC, NS, $\mathrm{ON}$ )

**Philonema sp. (AB, NT)

Raphidascaris acus (MB)

**Raphidascaris sp. (MB)

Spinitectus gracilis (MB, ON)
Sterliadochona ephemeridarum (ON)

Acanthocephala:

Echinorhynchus lateralis (ON)

Echinorhynchus leidyi (MB, NT)

Echinorhynchus salmonis $(\mathrm{AB}, \mathrm{MB}$, NT, ON)

Echinorhynchus sp. (NT)

Neoechinorhynchus rutili (SK)

Neoechinorhynchus tumidus (ON, SK)

Neoechinorhynchus sp. (ON)

Pomphorhynchus bulbocolli (AB,

MB)

Annelida:

Piscicola geometra (QC, SK)

Piscicola milneri (MB)

Piscicola punctata (ON)

Mollusca:

*Unionidae gen. sp. (ON)

Branchiura:

Argulus appendiculosus (MB)

Argulus stizostethi (MB, ON, SK)

Argulus sp. (ON)

Copepoda:

Ergasilus auritus (AB)

Ergasilus caeruleus $(\mathrm{ON})$

Ergasilus luciopercarum (MB)

Ergasilus nerkae (AB, $\mathrm{BC}, \mathrm{MB})$

Ergasilus sp. (AB, ON)

Salmincola corpulentus (NT, ON, QC)

Salmincola extensus (AB, MB, ON) 
Coregonus clupeaformis (Mitchill)

lake whitefish

Monogenea:

Discocotyle sagittata (AB, LB, MB, NF, ON, YT)

Tetraonchus variabilis (BC)

Digenea:

Crepidostomum auriculatum (AB)

Crepidostomum cooperi (AB, LB, ON)

Crepidostomum farionis (AB, $\mathrm{BC}$, LB, MB, ON, YT)

Crepidostomum metoecus (YT)

Crepidostomum sp. (AB)

Digenea gen. sp. (ON)

*Diplostomum baeri bucculentum (BC, MB)

*Diplostomum spathaceum (AB, LB, MB, ON, YT)

*Diplostomum spathaceum indistinctum (MB, ON)

*Ichthyocotylurus erraticus (AB, MB, $\mathrm{ON}$ )

*Neascus sp. (MB)

Phyllodistomum coregoni (AB, LB, ON)

Phyllodistomum umblae (LB)

*Tetracotyle sp. (AB, BC, YT)

*Tylodelphys scheuringi (BC)

Cestoidea:

Bothrimonas sturionis (MB, NB, NT)

${ }^{\star}$ Bothriocephalus sp. (AB, NT, ON)

Cestoidea gen. sp. (AB, BC, MB, NT, ON, SK, YT)

Cyathocephalus truncatus $(\mathrm{AB}, \mathrm{MB}$, NT, ON, YT)
*Diphyllobothrium dendriticum (AB, $\mathrm{BC}, \mathrm{MB}$ )

*Diphyllobothrium sp. (AB, BC, LB, MB, NT, ON, YT)

Eubothrium crassum (ON)

Eubothrium salvelini (AB, ON, YT)

Eubothrium sp. (LB, NT)

**Ligula sp. (MB)

Proteocephalus coregoni (MB, NT)

Proteocephalus filicollis (MB)

Proteocephalus longicollis (AB, BC,

LB, MB, ON, SK, YT)

Proteocephalus singularis (AB, MB)

Proteocephalus sp. (AB, BC, LB, MB, NT, ON, QC, SK)

*Schistocephalus sp. (ON)

*Triaenophorus crassus (AB, CA, EXP, LB, MB, NT, ON, QC, SK, YT)

*Triaenophorus sp. (AB, MB, NT, QC, SK)

Nematoda:

*Contracaecum sp. (AB)

Cystidicola farionis (AB, BC, MB, NT, ON, QC, SK, YT)

Cystidicola serrata (ON)

Cystidicola stigmatura (AB, MB, NT, ON, SK)

Cystidicola sp. (AB, BC, MB, ON)

**Hysterothylacium sp. (BC)

**Nematoda gen. sp. (AB, NB, NT, ON, SK)

**Philometra sp. (ON)

Philonema agubernaculum (AB, BC, LB, NT)

**Philonema oncorhynchi (BC, NS, $\mathrm{ON}$ )

**Philonema sp. (BC)

Pseudocapillaria (Ichthyocapillaria) salvelini (LB, ON) 
Pseudocapillaria (Pseudocapillaria) tomentosa (ON)

${ }^{*}$ Raphidascaris acus (AB, ON, YT)

Raphidascaris acus (AB, LB, MB, QC)

**Raphidascaris sp. (AB, LB, MB)

Rhabdochona sp. (ON)

Spinitectus gracilis (MB, ON)

Sterliadochona ephemeridarum (BC, LB, ON)

Truttaedacnitis truttae (BC)

Acanthocephala:

Acanthocephala gen. sp. (AB, MB) Acanthocephalus dirus (ON) Acanthocephalus sp. (MB)

Echinorhynchus gadi (NT)

Echinorhynchus lateralis (LB, NF, ON)

Echinorhynchus leidyi (EXP, MB, NT, ON, SK)

Echinorhynchus salmonis (AB, BC, MB, NT, ON)

Echinorhynchus sp. (NT, ON)

Leptorhynchoides thecatus $(\mathrm{ON})$

Neoechinorhynchus cylindratus (BC, MB)

Neoechinorhynchus rutili (BC)

Neoechinorhynchus salmonis (BC)

Neoechinorhynchus tumidus (AB, NT, ON, SK, YT)

Neoechinorhynchus sp. (AB)

Pomphorhynchus bulbocolli (AB, $\mathrm{BC}, \mathrm{MB}, \mathrm{ON}$ )

Pomphorhynchus sp. (MB)

Annelida:

Piscicola geometra (QC, SK)

Piscicola milneri (MB, ON, YT)

Piscicola punctata (ON)
Mollusca:

*Unionidae gen. sp. $(\mathrm{AB}, \mathrm{ON})$

Branchiura:

Argulus biramosus (AB)

Argulus stizostethi (MB, SK)

Copepoda:

Achtheres pimelodi (ON)

Coregonicola orientalis (NT)

Coregonicola sp. (NT)

Ergasilus auritus (AB)

Ergasilus caeruleus $(\mathrm{ON})$

Ergasilus elongatus $(\mathrm{ON})$

Ergasilus nerkae (AB, BC, MB)

Ergasilus sp. (AB, ON)

Salmincola coregonorum (LB)

Salmincola corpulentus (AB, LB, $\mathrm{MB}, \mathrm{NT}, \mathrm{ON}$ )

Salmincola extensus (AB, LB, MB, ON, YT)

Salmincola thymalli $(\mathrm{BC})$

Samincola sp. (MB)

Arachnoidea:

*Unionicola sp. (BC)

Coregonus zenithicus (Jordan and

Evermann) shortjaw cisco

Cestoidea:

Cyathocephalus truncatus $(\mathrm{AB}, \mathrm{MB}$, ON)

*Diphyllobothrium dendriticum (ON)

*Diphyllobothrium latum (ON)

*Diphyllobothrium sp. (ON) 
Proteocephalus longicollis (MB, $\mathrm{ON}$, SK)

*Triaenophorus crassus (AB, MB, ON, SK)

*Triaenophorus sp. (AB, MB, SK)

Coregonus sp.

Cestoidea:

Bothriocephalus cuspidatus (HBD)

*Diphyllobothrium sp. (NT)

Eubothrium crassum (NT)

Proteocephalus coregoni (HBD, MB)

Proteocephalus longicollis (NT)

*Triaenophorus crassus $(\mathrm{AB}, \mathrm{MB}$, NT, ON, SK)

*Triaenophorus sp. (AB, HBD)

Nematoda:

Cystidicola farionis (ON)

Philonema sp. (NT)

Acanthocephala:

Echinorhynchus salmonis (NT)

Branchiura:

Argulus stizostethi (ON)

Copepoda:

Salmincola corpulentus (NT, ON, QC)

Oncorhynchus clarki (Richardson) cutthroat trout
Gyrodactyloidea gen. sp. (BC)

Gyrodactylus salmonis (BC, NS)

Gyrodactylus sp. (BC)

Digenea:

*Clinostomum complanatum (BC)

${ }^{*}$ Clinostomum sp. (BC)

Crepidostomum farionis (AB, $\mathrm{BC})$

Crepidostomum metoecus (BC)

* Diplostomum sp. (BC)

${ }^{*}$ Neascus sp. (BC)

Cestoidea:

*Diphyllobothrium dendriticum (AB)

*Diphyllobothrium sp. (AB, BC)

Eubothrium salvelini (BC)

Proteocephalus longicollis (BC)

Nematoda:

*Contracaecum sp. (BC)

*Eustrongylides sp. (BC)

**Philonema oncorhynchi (BC, NS, ON)

**Philonema sp. (BC)

Pseudocapillaria (Pseudocapillaria) tomentosa (BC)

Rhabdochona cascarilla (BC)

Rhabdochona milleri ( $\mathrm{AB}, \mathrm{BC})$

Rhabdochona sp. (AB)

Salvelinema walkeri (BC)

Sterliadochona ephemeridarum (BC)

Truttaedacnitis truttae (BC)

Acanthocephala:

Neoechinorhynchus rutili (BC)

Pomphorhynchus bulbocolli (BC)

Monogenea:

Copepoda: 
Salmincola californiensis (BC)

Salmincola edwardsi (BC)

Oncorhynchus kisutch (Walbaum)

coho salmon

Monogenea:

Discocotyle sagittata (AB)

Gyrodactyloidea gen. sp. (BC)

Gyrodactylus salmonis (BC)

Monogenea gen. sp. (BC)

Tetraonchus alaskensis (BC)

Digenea:

Crepidostomum farionis (AB, BC)

Crepidostomum metoecus (BC)

*Diplostomum spathaceum (ON)

*Diplostomum sp. (BC)

*Ichthyocotylurus platycephalus (BC)

${ }^{*}$ Neascus sp. (BC)

*Strigeidae gen. sp. (BC)

Cestoidea:

Cyathocephalus truncatus (AB)

*Diphyllobothrium sp. (AB, BC)

Eubothrium salvelini $(\mathrm{AB}, \mathrm{BC})$

Eubothrium sp. (AB)

*Phyllobothrium caudatum (BC)

*Phyllobothrium sp. (BC)

Proteocephalus longicollis (BC)

**Proteocephalus sp. (AB, BC)

*Schistocephalus solidus (AB)

*Triaenophorus nodulosus (AB)

Nematoda:

Camallanus oxycephalus (ON)

Cystidicola farionis (AB, ON)
*Eustrongylides sp. (BC)

**Nematoda gen. sp. (BC)

Philonema agubernaculum (AB, $\mathrm{BC})$

**Philonema oncorhynchi (BC)

Pseudocapillaria (Ichthyocapillaria) salvelini $(\mathrm{BC}, \mathrm{ON})$

Pseudocapillaria (Pseudocapillaria) tomentosa (BC)

Rhabdochona kisutchi (BC)

Rhabdochona sp. (BC)

Salvelinema walkeri (BC)

Sterliadochona ephemeridarum (BC, ON)

Acanthocephala:

Acanthocephalus dirus (ON)

Echinorhynchus lateralis (ON)

Echinorhynchus salmonis $(\mathrm{AB}, \mathrm{ON})$

Neoechinorhynchus rutili (BC)

Neoechinorhynchus saginatus (BC)

Neoechinorhynchus salmonis (BC)

Neoechinorhynchus strigosus (AB)

Pomphorhynchus bulbocolli (AB, BC)

Rhadinorhynchus trachuri (BC)

Annelida:

Piscicola snlmositica

Copepoda:

Ergasilus auritus $(\mathrm{AB})$

Ergasilus nerkae (AB, $\mathrm{BC})$

Salmincola californiensis (BC)

Oncorhynchus mykiss (Richardson) rainbow trout

Monogenea: 
Discocotyle sagittata (ON)

Gyrodactyloidea gen. sp. (BC)

Gyrodactylus avalonia (NS)

Gyrodactylus colemanensis (BC, EXP,

$\mathrm{NF}, \mathrm{NS}, \mathrm{ON}$ )

Gyradactylus salmonis (BC, EXP, NS)

Gyrodactylus sp. (AB, BC, NS)

Monogenea gen. sp. (AB)

Tetraonchus variabilis (BC)

Digenea:

Allocreadium lobatum (BC)

Bunodera luciopercae ( $\mathrm{AB}, \mathrm{BC})$

*Clinostomum complanatum (BC, $\mathrm{MB}$ )

*Clinostomum sp. (BC, MB)

Crepidostomum farionis $(\mathrm{AB}, \mathrm{BC}$, $\mathrm{MB}, \mathrm{NF}, \mathrm{NT}, \mathrm{QC})$

Crepidostomum sp. (AB)

Digenea gen. sp. (AB)

*Diplostomum baeri bucculentum (BC)

*Diplostomum spathaceum (BC)

*Diplostomum sp. (BC, MB, ON)

*Ichthyocotylurus erraticus (ON)

*Neascus sp. (BC)

Phyllodistomum umblae (BC, ON)

Phyllodistomum sp. (BC)

* Tetracotyle sp. (BC, NF, ON)

*Tylodelphys scheuringi (BC, MB)

\section{Cestoidea:}

Cestoidea gen. sp. (BC)

Cyathocephalus truncatus (BC)

* Diphyllobothrium dendriticum (BC, QC)

*Diphyllobothrium ditremum (BC, QC)

**Diphyllobothrium latum (AB)

*Diphyllobothrium sp. (AB, BC, NF,
ON)

Eubothrium crassum (ON)

Eubothrium salvelini (AB, BC, NF)

Eubothrium sp. (AB)

*Paradilepis simoni (BC)

Proteocephalus longicollis (BC)

Proteocephalus sp. (BC, ON, SK)

*Schistocephalus sp. (BC)

*Triaenophorus crassus (EXP, MB)

Nematoda:

Capillariidae gen. sp. (BC)

Capillospirura pseudoargumentosus (EXP, ON)

* Contracaecum sp. (ON)

*Cosmocephalus obvelatus (EXP, ON)

Cystidicola farionis (AB, BC, EXP, NT, ON, QC, YT)

Cystidicola stigmatura $(\mathrm{AB}, \mathrm{BC})$

Cystidicola sp. (BC, ON)

*Eustrongylides sp. (BC)

Haplonema hamulatum (BC)

**Hysterothylacium sp. (BC)

**Nematoda gen. sp. $(A B, B C)$

Philonema agubernaculum (AB, BC, NF, NT)

*Philonema oncorhynchi (BC)

Philonema oncorhynchi (BC, EXP)

**Philonema sp. (BC)

Pseudocapillaria (Ichthyocapillaria) salvelini (CN)

Pseudocapillaria (Pseudocapillaria) tomentosa (BC)

Raphidascaris acus (EXP, ON, QC)

Rhabdochona canadensis (AB)

Rhabdochona cascadilla (BC)

Rhabdochona kisutchi (BC)

Rhabdochona milleri ( $\mathrm{AB}, \mathrm{BC})$

Rhabdochona sp. (BC)

Spiruridae gen. sp. (BC)

Sterliadochona ephemeridarum (BC, 
ON)

Truttaedacnitis truttae (AB, $\mathrm{BC})$

Acanthocephala:

Acanthocephala gen. sp. (NF)

Acanthocephalus dirus (ON)

Echinorhynchus lateralis (NF, ON)

Echinorhynchus leidyi (EXP, ON)

Echinorhynchus salmonis (ON)

Neoechinorhynchus rutili (BC, MB, ON)

Neoechinorhynchus saginatus (BC)

Neoechinorhynchus salmonis (BC)

Neoechinorhynchus sp. (BC)

Pomphorhynchus bulbocolli (AB, $\mathrm{BC}, \mathrm{MB}, \mathrm{ON}$ )

Annelida:

Desserobdella phalera (EXP, ON)

Illinobdella sp. (BC)

Myzobdella sp. (BC)

Piscicola milneri ( $\mathrm{AB})$

Mollusca:

*Unionidae gen. sp. (BC)

Copepoda:

Copepoda gen. sp. (NF)

Ergasilus nerkae ( $\mathrm{AB}, \mathrm{BC}, \mathrm{NF})$

Salmincola californensis (BC)

Salmincola edwardsii (BC, NF)

Arachnoidea:

*Unionicola sp. (BC)

Prosopium coulteri (Eigenmann and
Eigenmann) pygmy whitefish

Monogenea:

Tetraonchus variabilis (BC)

Nematoda:

Cystidicola sp. (BC)

Rhabdochona milleri (BC)

Acanthocephala:

Neoechinorhynchus rutili (BC)

Prosopium cylindraceum (Pallas)

round whitefish

Monogenea:

Discocotyle sagittata (LB, ON, YT)

Gyrodactyloidea gen. sp. (BC)

Tetraonchus loftusi (ON)

Tetraonchus variabilis (LB, ON, YT)

Digenea:

*Apophallus brevis (ON)

Bunodera luciopercae (LB)

Crepidostomum cooperi (ON)

Crepidostnmum farionis (BC, NT, ON, $\mathrm{QC}, \mathrm{YT}$ )

*Diplostomum spathaceum (LB, ON, YT)

*Diplostomum sp. (BC, ON, QC)

Ichthyocotylurus erraticus (ON)

Phyllodistomum coregoni (LB)

Phyllodistomum sp. (ON)

*Tetracotyle sp. (ON, YT)

Cestoidea: 
Cyathocephalus truncatus (ON, YT)

*Diphyllobothrium sp. (NS, YT)

Eubothrium salvelini (LB, NF, ON, YT)

Eubothrium sp. (LB)

Proteocephalus longicollis $(\mathrm{CA}, \mathrm{ON}$, YT)

Proteocephalus sp. (AB, BC, LB)

*Triaenophorus crassus $(\mathrm{AB}, \mathrm{MB}$, NT, ON, QC, SK, YT)

*Triaenophorus nodulosus (ON)

Nematoda:

Cystidicola farionis (AB, BC, NT, ON, QC, YT)

Cystidicola stigmatura (BC, ON, SK)

*Eustrongylides sp. (BC)

**Nematoda gen. sp. (LB)

**Philometra sp. (ON)

Philonema agubernaculum (LB)

**Philonema sp. (BC)

Pseudocapillaria (Ichthyocapillaria) salvelini (LB, ON, YT)

Spinitectus gracilis (ON)

Sterliadochona ephemeridarum (BC)

Truttaedacnitis truttae (BC)

Acanthocephala:

Acanthocephalus dirus (ON)

Echinorhynchus lateralis (LB, ON)

Echinorhynchus salmonis (ON)

Neoechinorhynchus tumidus (ON, YT)

Pomphorhynchus bulbocolli (ON)

Annelida:

Piscicola milneri (ON, YT)

Mollusca:
*Unionidae gen. sp. (ON)

Copepoda:

Ergasilus caeruleus $(\mathrm{ON})$

Ergasilus luciopercarum (LB)

Ergasilus sp. (ON)

Salmincola corpulentus (ON)

Salmincola extensus (ON)

Salmincola thymalli (LB, ON)

Salmincola sp. (ON)

Prosopium williamsoni (Girard) mountain whitefish

Monogenea:

Discocotyle sagittata (BC)

Gyrodactyloidea gen. sp. (BC)

Tetraonchus variabilis (BC)

Digenea:

Allocreadium lobatum (BC)

Crepidostomum farionis $(\mathrm{AB}, \mathrm{BC}$, MB)

*Diplostomum baeri bucculentum (BC)

*Diplostomum spathaceum (BC)

*Diplostomum sp. (BC)

*Ichthyocotylurus platycephalus (BC)

*Strigeidae gen. sp. (BC, NB)

*Tetracotyle sp. (BC, $\mathrm{ON})$

*Tylodelphys scheuringi (BC)

Cestoidea:

*Diphyllobothrium dendriticum (BC)

*Diphyllobothrium sp. (AB, BC)

Eubothrium salvelini (AB, BC, NT)

Eubothrium sp. (BC) 
${ }^{*}$ Ligula intestinalis $(\mathrm{BC})$

*Paradilepis simoni (BC)

Proteocephalus longicollis $(\mathrm{BC})$

Proteocephalus sp. (AB, BC, ON)

${ }^{*}$ Schistocephalus sp. (BC)

*Triaenophorus crassus (AB, NT)

Nematoda:

*Anisakidae gen. sp. (BC)

Cystidicola farionis (BC, $\mathrm{ON}$ )

Cystidicola stigmatura (AB, BC, $\mathrm{ON}$ )

Cystidicola sp. (AB)

*Eustrongylides sp. (BC)

Haplonema hamulatum (BC)

**Hysterothylacium sp. (BC)

**Nematoda gen. sp. (AB, BC)

Philonema agubernaculum (AB, $\mathrm{BC}$,

NT)

**Philonema sp. (BC)

Pseudocapillaria (Pseudocapillaria) tomentosa (BC)

Rhabdochona cascadilla (BC)

Rhabdochona kisutchi (BC)

Rhabdochona milleri (BC)

Rhabdochona sp. (BC)

Sterliadochona ephemeridarum (BC)

Truttaedacnitis truttae $(\mathrm{AB}, \mathrm{BC})$

Acanthocephala:

Neoechinorhynchus rutili (BC)

Neoechinorhynchus saginatus (BC)

Neoechinorhynchus salmonis (BC)

Neoechinorhynchus tumidus (YT)

Neoechinorhynchus sp. (BC)

Pomphorhynchus bulbocolli (BC)

Annelida:

Hirudinea gen. sp. (BC)

Piscicola sp. (BC)
Rhynchobdellidae gen. sp. (BC)

Copepoda:

Achtheres sp. (AB)

Coregonicola sp. (AB)

Ergasilus nerkae (BC)

Salmincola californiensis (BC)

Salmincola edwardsii (BC)

Salmincola extensus (BC)

Salmincola thymalli (BC)

Salmincola sp. (BC)

Arachnoidea:

*Unionicola sp. (BC)

Prosopium sp.

Cestoidea:

*Triaenophorus crassus (AB)

Copepoda:

Salmincola thymalli (NT)

Salmon trutta Linnaeus brown trout

Monogenea:

Discocotyle sagittata (NF, ON)

Gyrodactylus colemanensis (ON)

Digenea:

*Apophallus brevis (NF, QC)

Crepidostomum farionis (NF)

Cestoidea: 
Bothrimonas sturionis (LB, NB)

*Diphyllobothrium sp. (AB, NF)

Eubothrium salvelini (NF)

Proteocephalus parallacticus (ON)

Nematoda:

Cystidicola farionis (ON)

Sterliadochona ephemeridarum (NF,

ON)

Acanthocephala:

Acanthocephalus dirus (ON)

Echinorhynchus lateralis (NF)

Echinorhynchus salmonis (ON)

Annelida:

Johanssonia arctica (EXP)

Copepoda:

Ergasilus sp. (NF)

Salvelinus confluentus (Suckley)

bull charr

Cestoidea:

Cyathocephalus truncatus (AB)

Eubothrium salvelini (AB)

Glaridacris terebrans ( $\mathrm{AB})$

Cestoidea gen. sp. (AB)

Salvelinus fontinalis (Mitchill)

brook charr

Monogenea:
Discocotyle sagittata (LB, NF, NS, ON)

Discocotyle sp. (QC)

Gyrodactylus avalonia (NF)

Gyrodactylus calliaratus (NB)

Gyrodactylus colemanensis (EXP, NB, NS, ON)

Gyrodactylus perlucides (NB)

Gyrodactylus salmonis (EXP, BC, NB, NS)

Gyrodactylus sp. (LB, NS)

Tetraonchus variabilis (LB)

Digenea:

Allocreadiidae gen. sp. (LB, NF)

*Apophallus brevis (NB, NF, ON, QC)

*Apophallus imperator (NB, NF, NS, ON, QC)

*Apophallus sp. (ON, QC)

Azygia longa (NB, NF)

Bunodera luciopercae (AB, BC, LB, NF, ON)

*Clinostomum complanatum (ON, QC)

Crepidostomum cooperi (NF, QC)

Crepidostomum cornutum (NS, QC)

Crepidostomum farionis (AB, $\mathrm{BC}$, LB, NB, NF, NS, ON, QC)

Crepidostomum sp. (QC)

*Cryptocotyle lingua (NB)

*Digenea gen. sp. (AB, ON, QC)

Digenea gen. sp. (ON, QC)

*Diplostomum spathaceum (CA, EXP, LB, NS, ON, QC)

*Diplostomum sp. (BC, NB, NF, NS, ON, QC)

Gorgoderidae gen. sp. (NF)

${ }^{*}$ Heterophyidae gen. sp. (NF)

*Ichthyocotylurus erraticus (ON)

*Metorchis conjunctus (QC)

*Neascus sp. (BC, ON) 
Phyllodistomum superbum (QC)

Phyllodistomum umblae (LB, NF, ON, QC)

Pleurogenes sp. (QC)

Posthodiplostomum minimum (ON)

Ptychogonimus fontanus (QC)

*Strigeidae gen. sp. (ON)

*Tetracotyle sp. (BC, NF, ON, QC)

Cestoidea:

Bothrimonus sturionis (LB, MB, NT)

Cestoidea gen. sp. (NS, ON, QC)

Cyathocephalus truncatus (CA)

*Diphyllobothriidae gen. sp. (QC)

*Diphyllobothrium dendriticum (BC, QC)

*Diphyllobothrium ditremum (BC, CA, QC)

*Diphyllobothrium sp. (AB, BC, LB, $\mathrm{MB}, \mathrm{NB}, \mathrm{NF}, \mathrm{NS}, \mathrm{NT}, \mathrm{ON}, \mathrm{QC})$

Eubothrium crassum (NB)

Eubothrium salvelini $(\mathrm{AB}, \mathrm{BC}, \mathrm{HBD}$,

LB, MB, NF, ON, QC)

Eubothrium sp. (LB, NF, QC)

${ }^{*}$ Ligula intestinalis $(\mathrm{BC}, \mathrm{QC})$

*Ligula sp. (ON, QC)

Proteocephalidae gen. sp. (QC)

Proteocephalus ambloplitis (ON)

Proteocephalus longicollis (LB, QC)

Proteocephalus parallacticus (AB, ON, QC)

Proteocephalus sp. (AB, LB, NB, ON, QC, SK)

*Schistocephalus solidus (NF, QC)

Tetraphyllidea gen. sp. (NB)

Nematoda:

Contracaecum sp. (LB, QC)

Cystidicola farionis (ON, QC)

Cystidicola stigmatura (MB, $\mathrm{ON}$ )
*Eustrongylides sp. (NB)

**Hysterothylacium brachyurum (ON)

**Hysterothylacium sp. (QC)

**Nematoda gen. sp. (LB, NS, ON, QC)

**Philometra cylindracea (QC)

**Philometra sp. (QC)

Philonema agubernaculum (LB, NF, NT, QC)

**Philonema oncorhynchi (BC, NS, ON

**Philonema sp. (LB, QC)

Pseudocapillaria (Ichthyocapillaria) salvelini (LB, NB, NF, QC)

Pseudocapillaria (Pseudocapillaria) tomentosa (BC, ON)

Raphidascaris acus (EXP, ON, QC)

**Raphidascaris sp. (LB, NB)

Rhabdochona cascadilla (AB)

Rhabdochona milleri ( $\mathrm{AB}, \mathrm{BC})$

Rhabdochona ovifilementa (QC)

Rhabdochona sp. (AB, BC)

*Seuratinae gen. sp. (QC)

Sterliadochona ephemeridarum (BC, $\mathrm{LB}, \mathrm{NB}, \mathrm{NF}, \mathrm{NS}, \mathrm{ON}, \mathrm{QC})$

Truttaedacnitis truttae (BC, NB)

Truttaedacnitis sp. (BC)

Acanthocephala:

Acanthocephala gen. sp. (NF, ON, QC) Acanthocephalus dirus (ON)

Echinorhynchus gadi (QC)

Echinorhynchus lateralis (LB, NB, NS, ON, QC)

Echinorhynchus leidyi (QC)

Echinorhynchus salmonis (CA, ON)

Echinorhynchus sp. (LB, QC)

Leptorhynchoides thecatus (ON)

Neoechinorhynchus cylindratus (QC)

Neoechinorhynchus rutili (BC, $\mathrm{ON})$ 
Neoechinorhynchus sp. (NB)

Pomphorhynchus bulbocolli (QC)

Annelida:

Hirudinea gen. sp. (NF)

Piscicola milneri (AB, ON)

Piscicola punctata (AB, NB, ON, QC)

Mollusca:

*Anodonta cataracta (NF)

*Unionidae gen. sp. (NT, ON, QC)

Branchiura:

Argulus stizostethi (NF, NS)

Copepoda:

Ergasilus auritus (QC)

Ergasilus labracis (NB)

Ergasilus luciopercarum (NF)

Ergasilus sp. (NF)

Salmincola carpionis (NB, QC)

Salmincola edwardsii (BC, EXP, LB,

$\mathrm{MB}, \mathrm{NB}, \mathrm{NF}, \mathrm{NS}, \mathrm{ON}, \mathrm{PE}, \mathrm{QC})$

Salmincola sp. (MB, NF, QC)

Salvelimus fontinalis (Mitchill) X Salvelinus namaycush (Walbaum) splake

Monogenea:

Discocotyle sagittata $(\mathrm{ON})$

Gyrodactylus colemanensis (NF, NS, $\mathrm{ON}$ )

Digenea:

*Diplostomum spathaceum (ON)
*Diplostomum spathaceum indistinctum (ON)

*Ichthyocotylurus erraticus (MB, ON)

*Tetracotyle sp. (ON)

Cestoidea:

Cyathocephalus truncatus (ON)

*Diphyllobothrium sp. (AB, BC, ON)

*Eubothrium salvelini (ON)

Proteocephalus parallacticus (ON)

Proteocephalus sp. (ON, QC)

*Triacnophorus nodulosus (ON)

Nematoda:

Cystidicola farionis (ON)

Cystidicola stigmatura (ON)

**Hysterothylacium brachyurum (ON)

Pseudocapillaria (Ichthyocapillaria) salvelini (ON)

Rhabdochona sp. (ON)

Spinitectus gracilis $(\mathrm{ON})$

Acanthocephala:

Acanthocephalus dirus (ON)

Echinorhynchus salmonis (ON)

Neoechinorhynchus tumidus (ON)

Pomphorhynchus bulbocolli (ON)

Mollusca:

*Unionidae gen. sp. (ON)

Copepoda:

Ergasilus caeruleus $(\mathrm{ON})$

Ergasilus siscowet (ON) 
Monogenea:

Discocotyle sagittata (BC)

Gyrodactyloidea gen. sp. (BC)

Tetraonchus alaskensis (BC)

Tetraonchus variabilis (BC)

Digenea:

Bunodera luciopercae (BC)

Crepidostomum farionis (BC)

*Diplostomum baeri bucculentum (BC)

*Diplostomum spathaceum (BC)

*Iiplostomum sp. (BC)

*Neascus sp. (BC)

Prosorhynchoides ozakii (BC)

*Tetracotyle sp. (BC)

Cestoidea:

Bothriocephalus cuspidatus (AB, MB)

Cestoidea gen. sp. (AB, BC)

Cyathocephalus truncatus $(\mathrm{AB}, \mathrm{BC})$

*Diphyllobothrium dendriticum (BC, QC)

*Diphyllobothrium ditremum (BC, QC)

*Diphyllobothrium latum (AB, BC)

*Diphyllobothrium sp. (BC)

Eubothrium salvelini ( $\mathrm{AB}, \mathrm{BC})$

Glaridacris terebrans (AB)

*Phyllobothrium sp. (BC)

Proteocephalus longicollis (BC)

Proteocephalus sp. (AB, BC, ON)

Nematoda:

*Contracaecum sp. (BC)

Cystidicola farionis (BC, NT, ON, $\mathrm{QC}, \mathrm{YT}$ )
Cystidicola stigmatura (BC)

Cystidicola sp. (BC, ON)

*Eustrongylides sp. (BC)

Haplonema hamulatum (BC)

Hysterothylacium brachyurum (BC)

*Hysterothylacium sp. (BC)

Nematoda gen. sp. (AB, BC)

Philonema agubernaculum (BC, NT)

**Philonema oncorhynchi (BC)

**Philonema sp. (BC)

Pseudocapillaria (Ichthyocapillaria) salvelini (BC)

Pseudocapillaria (Pseudocapillaria) tomentosa (BC)

Rhabdochona canadensis (BC)

Rhabdochona kisutchi (BC)

Rhabdochona milleri (AB, $\mathrm{BC})$

Rhabdochona sp. (BC)

Salvelinema walkeri (BC)

Sterliadochona ephemeridarum (BC)

Truttaedacnitis truttae (BC)

Acanthocephala:

Echinorhynchus leidyi (NT, ON)

Echinorhynchus salmonis (NT)

Neoechinorhynchus rutili (BC)

Neoechinorhynchus salmonis (BC, NT)

\section{Copepoda:}

Salmincola californensis (BC, NT)

Salmincola carpionis (NT)

Salmincola edwardsii (BC, NF, ON)

Salmincola siscowet (NT)

Salvelimus namaycush (Walbaum)

lake char

Monogenea: 
Discocotyle sagittata (AB, LB, ON, YT)

Gyrodactylus colemanensis (BC, NF, $\mathrm{NS}, \mathrm{ON}$ )

Tetraonchus monenteron (LB)

Digenea:

*Apophallus sp. (ON)

Azygia angusticauda (ON)

Azygia longa (ON)

Bunodera luciopercae (BC, LB)

Crepidostomum cooperi (LB)

Crepidostomum farionis (AB, BC,

LB, NT, ON, YT)

Crepidostomum metoecus (YT)

*Diplostomum baeri bucculentum (BC)

*Diplostomum spathaceum (AB, LB, ON, YT)

*Diplostomum sp. (BC, ON, QC)

Phyllodistomum umblae (LB)

Phyllodistomum sp. (BC)

*Tetracotyle sp. (BC, YT)

Cestoidea:

Bothrimonus sturionis (NB, NT)

Bothriocephalus cuspidatus (AB, MB)

Bothriocephalus sp. (AB, MB)

*Cestoidea gen. sp. (AB)

Cestoidea gen. sp. (AB, NS, NT, ON)

Cyathocephalus truncatus $(\mathrm{AB}, \mathrm{NT}$, YT)

*Diphyllobothrium dendriticum (BC, $\mathrm{MB}, \mathrm{NT}, \mathrm{ON}, \mathrm{QC})$

*Diphyllobothrium ditremum (BC, CA, NT, QC)

*Diphyllobothrium latum (MB)

*Diphyllobothrium sp. (AB, BC, LB, MB, NT, ON, YT)
Eubothrium crassum (LB, NT, ON)

Eubothrium salvelini (AB, BC, LB, MB, NB, NF, NT, ON, QC, SK, YT) Eubothrium sp. (LB, MB, QC)

Glaridacris catostomi (SK)

Proteocephalidae gen. sp. (QC)

Proteocephalus ambloplitis (ON)

Proteocephalus coregoni (MB)

Proteocephalus longicollis (LB, NT, ON, YT)

Proteocephalus parallacticus (AB, ON)

Proteocephalus sp. (AB, BC, LB, NT, ON, QC, YT)

*Schistocephialus solidus (NT, SK)

*Triaenophorus crassus (AB, LB, MB, NT, ON, SK, YT)

*Triaenophorus nodulosus (AB, MB, NT)

*Triaenophorus sp. (AB, MB, NT)

Nematoda:

Cystidicola farionis (AB, BC, MB, NT, ON, QC, SK, YT)

Cystidicola stigmatura (AB, BC, EXP, $\mathrm{MB}, \mathrm{NT}, \mathrm{ON}, \mathrm{QC}, \mathrm{SK}$ )

Cystidicola sp. (AB, BC, MB, ON)

*Eustrongylides sp. (BC)

Hysterothylacium brachyurum (AB)

*Nematoda gen. sp. (AB, LB, MB, NT, ON, SK)

Philonema agubernaculum (AB, BC, LB, NT)

* *Philonema oncorhynchi (BC, NS, ON)

*Philonema sp. (AB, BC, NT, ON)

Pseudocapillaria (Ichthyocapillaria) salvelini (BC, LB, NB, NF, ON, YT)

*Raphidascaris acus (AB)

* Raphidascaris sp. (AB)

Raphidascaris sp. (LB) 
Sterliadochona ephemeridarum (LB, ON)

Truttaedacnitis truttae $(\mathrm{AB}, \mathrm{BC})$

Acanthocephala:

Acanthocephala gen. sp. (AB, NT)

Acanthocephalus dirus (ON)

Echinorhynchus gadi (NT)

Echinorhynchus lateralis (LB, ON)

Echinorhynchus leidyi (AB, MB, NT, ON, QC, SK)

Echinor hynchus salmonis (AB, MB, NT, ON)

Echinorhynchus sp. (NT)

Leptorhynchoides thecatus (ON)

Neoechinorhynchus rutili (BC, YT)

Neoechinorhynchus salmonis (BC)

Neoechinorhynchus sp. (BC)

Pomphorhynchus bulbocolli (AB)

Annelida:

Piscicola geometra (QC, SK)

Piscicola milneri (AB, NT, ON, QC, YT)

Copepoda:

Ergasilus auritus $(\mathrm{AB})$

Ergasilus elongatus $(\mathrm{ON})$

Ergasilus nerkae (AB)

Salmincola californiensis (BC)

Salmincola corpulentus (NT)

Salmincola edwardsii (BC, NB, NT,

ON, YT)

Salmincola siscowet (BC, LB, NT, ON, QC, SK)

Salmincola sp. (BC, MB, NT, SK)

Thymallus arcticus (Pallas) Arctic grayling
Monogenea:

Discocotyle sagittata (YT)

Gyrodactyloidea gen. sp. (BC)

Tetraonchus borealis (BC, SK, YT)

Digenea:

Allocreadium isoporum (YT)

Allocreadium lobatum (YT)

Crepidostomum farionis (BC, NT, YT)

Crepidostomum metoecus (YT)

Crepidostomum sp. (BC)

*Diplostomum spathaceum (YT)

*Diplostomum sp. (BC)

*Tetracotyle sp. (BC, YT)

Cestoidea:

Cyathocephalus sp. (YT)

*Diphyllobothrium sp. (AB, BC, YT)

Eubothrium salvelini (NT, YT)

Proteocephalus longicollis (YT)

*Triaenophorus crassus (AB, MB, NT, SK, YT)

*Triaenophorus nodulosus (NT)

Nematoda:

Cystidicola farionis (YT)

Metabronema sp. (BC)

Nematoda gen. sp. (NT)

Philonema sp. (BC)

Pseudocapillaria (Ichthyocapillaria) salvelini (YT)

*Raphidascaris acus (YT)

Rhabdochona cascadilla (BC)

Sterliadochona ephemeridarum (BC)

Acanthocephala:

Echinorhynchus salmonis (NT) 
Echinorhynchus sp. (YT)

Neoechinorhynchus rutili (BC, NT)

Neoechinorhynchus tumidus (YT)

Copepoda:

Ergasilus nerkae (BC)

Salmincola thymalli (BC, NT, YT)

Salmonidae gen. sp.

Monogenea:

Discocotyle sagittata (AB)

Tetraonchus variabilis (ON)

Digenea:

Crepidostomum farionis (AB)

*Diplostomum sp. (BC)

Cestoidea:

Cyathocephalus truncatus (AB)

*Diphyllobothrium sp. (AB)

Eubothrium salvelini ( $\mathrm{AB}, \mathrm{ON})$

Proteocephalus sp. (AB)

*Triaenophorus nodulosus (AB)

Nematoda:

Cystidicola farionis (AB, ON)

Philonema agubernaculum (AB)

Acanthocephala:

Echinorhynchus salmonis (AB)

Pomphorhynchus bulbocolli (AB)

Copepoda:
Ergasilus auritus $(\mathrm{AB})$

Ergasilus nerkae (AB)

ORDER PERCOPSIFORMES

FAMILY PERCOPSIDAE - trout perches

Percopsis omiscomaycus (Walbaum) trout-perch

Monogenea:

Cleidodiscus sp. (ON)

Gyrodactyoidea gen. sp. (ON)

Gyrodactylus sp. (ON)

Urocleidus adspectus (MB)

Urocleidus baldwini (ON)

Digenea:

Bucephalus sp. (ON)

*Centrovarium lobotes (MB, ON)

Centrovarium lobotes (ON)

*Clinostomum complanatum (ON)

Crepidostomum cooperi (MB)

Crepidostomum isostomum (ON)

Crepidostomum percopsisi (MB)

Digenea gen. sp. (MB)

*Diplostomum spathaceum (ON)

*Diplostomum spathaceum indistinctum (ON)

*Diplostomum sp. (MB, ON, SK)

*Ichthyocotylurus erraticus (ON)

*Ichthyocotylurus pileatus (ON)

*Ichthyocotylurus platycephalus (ON)

*Neascus sp. (MB, ON)

*Posthodiplostomum minimum (ON)

*Tetracotyle sp. (MB, ON)

*Tylodelphys scheuringi (ON)

Cestoidea: 
Bothriocephalus claviceps (ON) Bothriocephalus cuspidatus (ON) Bothriocephalus formosus (ON)

Proteocephalus pearsei (ON)

*Triaenophorus nodulosus (ON)

*Triaenophorus stizostedionis (AB, $\mathrm{MB}, \mathrm{ON}$ )

*Triaenophorus sp. (AB, ON)

Nematoda:

Camallamus oxycephalus (ON) Hysterothylacium brachyurum (ON) **Nematoda gen. sp. (ON)

**Raphidascaris acus (MB) Rhabdochona canadensis (MB) Rhabdochona cascadilla (ON) Rhabdochona sp. (ON) Spinitectus gracilis (MB, ON)

Acanthocephala:

Acanthocephalus dirus (ON) Echinorhynchus salmonis (ON) Leptorhynchoides thecatus (ON) Neoechinorhynchus sp. (ON) Pomphorhynchus bulbocolli (ON)

Annelida:

Illinobdella sp. (ON) Myzobdella lugubris (MB) Myzobdella sp. (ON) Piscicola punctata (ON)

Mollusca:

*Unionidae gen. sp. (ON)

Branchiura:

Argulus appendiculosus (MB)
Argulus versicolor (ON)

Argulus sp. (ON)

Copepoda:

Ergasilus caeruleus (ON)

Ergasilus lucioperculum (MB)

ORDER GADIFORMES

FAMILY GADIDAE - cods

Lota lota (Linneaus) burbot

Monogenea:

Gyrodactylus lotae (ON)

Gyrodactylus sp. (ON)

Digenea:

Azygia angusticauda (ON, QC) Azygia longa (ON, QC) Bunodera luciopercae (ON) Bunodera sacculata (ON) Crepidostomum cooperi (LB)

Crepidostomum farionis $(\mathrm{AB}, \mathrm{BC}$, NT)

Crepidostomum metoecus (YT)

Crepidostomum sp. (BC, ON)

*Digenea gen. sp. (MB)

Digenea gen. sp. (AB, MB)

* Diplostomum baeri bucculentum (BC, MB)

*Diplostomum spathaceum (BC, ON, YT)

*Diplostomum spathaceum indistinctum $(\mathrm{AB})$

*Diplstomum sp. (BC, ON, QC) 
Microphallidae gen. sp. (ON)

*Neascus sp. (MB, ON)

*Posthodiplostomum minimum (BC, ON)

*Tetracotyle sp. (ON, YT)

*Tylodelphys scheuringi (AB, BC)

Cestoidea:

Bothriocephalus sp. (BC, ON)

Cyathocephalus truncatus (AB)

*Diphyllobothriidae gen. sp. (MB)

*Diphyllobothrium dendriticum (BC, ON, QC)

*Diphyllobothrium latum (MB, QC)

*Diphyllobothrium sp. (BC, MB, NT, ON, QC, YT)

Eubothrium crassum (ON)

Eubothrium rugosum (AB, BC, MB, NT, ON)

Eubothrium sp. (MB)

*Ligula sp. (AB)

Proteocephalidae gen. sp. (AB)

Proteocephalus pearsei (ON)

Proteocephalus sp. (AB, BC, LB, NB, ON, QC)

Triaenophorus crassus (ON)

*Triaenophorus nodulosus (AB, MB, $\mathrm{ON}$ )

Triaenophorus nodulosus (YT)

Nematoda:

Camallanus oxycephalus (ON)

Cucullanellus cotylophora (ON)

*Eustrongylides sp. (BC)

Haplonema hamulatum $(\mathrm{AB}, \mathrm{BC}$, ON, YT)

Haplonema sp. (AB, LB)

*Hysterothylacium brachyurum (ON)

**Nematoda gen. sp. (AB, MB, NT)

Pseudocapillaria (Pseudocapillaria) tomentosa (ON)

*Raphidascaris acus (ON)

Raphidascaris acus (ON, QC)

Rhabdochona cascadilla (ON)

Spinitectus gracilis (ON)

Acanthocephala:

Acanthocephala gen. sp. (MB)

Acanthocephalus dirus (ON)

Echinorhynchus leidyi (MB, NT, ON)

Echinorhynchus salmonis (AB, BC, $\mathrm{MB}, \mathrm{NT}, \mathrm{ON}$ )

Echinorhynchus sp. (ON)

Leptorhynchoides thecatus (ON)

Neoechinorhynchus cylindratus (ON)

Neoechinorhynchus rutili (BC, ON, YT)

Neoechinorhynchus saginatus $(\mathrm{BC})$

Neoechinorhynchus salmonis (BC)

Pomphorhynchus bulbocolli (AB, $\mathrm{BC}, \mathrm{ON}$ )

Annelida:

Cystobranchus mammilatus (NT)

Cystobranchus verrilli $(\mathrm{AB}, \mathrm{ON})$

Myzobdella lugubris (ON)

Piscicola milneri (YT)

Piscicola punctata (ON)

Mollusca:

*Unionidae gen. sp. (ON)

Copepoda:

Ergasilus caeruleus $(\mathrm{BC}, \mathrm{ON})$

Ergasilus celestis $(\mathrm{ON})$

Ergasilus nerkae (BC)

Salmincola lotae (NT) 
ORDER GASTEROSTEIFORMES

FAMILY GASTEROSTEIDAE sticklebacks

Culaea inconstans (Kirtland)

brook stickleback

Monogenea:

Dactvlogyrus eucalius (ON)

Dactylogyrus sp. (ON)

Gyrodactyloidea gen. sp. (BC, ON)

Gyrodactylus eucaliae (ON)

Gyrodactylus sp. (ON)

Octomacrum lanceatum (ON)

Digenea:

*Apatemon gracilis (BC, QC)

Bunodera eucaliae (BC, ON, QC)

*Clinostomum complanatum (ON)

Culaeatrema inconstans (ON)

Digenea gen. sp. (ON)

*Diplostomum scudderi (ON)

*Diplostomum spathaceum (ON)

* Diplostomum sp. (ON)

*Neascus sp. (ON)

*Posthodiplostomum minimum (BC, ON)

*Tetracotyle sp. (BC, ON)

Cestoidea:

*Diphyllobothrium sp. (ON)

${ }^{*}$ Ligula intestinalis (ON)

${ }^{*}$ Ligula sp. (AB, MB)

*Proteocephalus ambloplitis (ON)

Proteocephalus pugetensis (ON)

Proteocephalus stizostethi (ON)
Proteocephalus sp. (ON)

*Schistocephalus solidus (AB, MB, ON)

*Schistocephalus sp. (AB, BC)

Nematoda:

Camallamus oxycephalus (ON)

*Contracaecum sp. (MB)

*Eustrongylides tubifex (ON)

* Eustrongylides sp. (ON)

*Hysterothylacium brachyurum (ON)

**Hysterothylacium sp. (ON)

**Nematoda gen. sp. (ON)

* Paracuaria adunca (ON)

Rhabdochona cascadilla (ON)

*Spiroxys sp. (ON)

Acanthocephala:

Acanthocephalus dirus (ON)

Leptorhynchoides thecatus (ON)

Neoechinorhynchus pungitius (ON)

Neoechinorhynchus rutili (ON)

Neoechinorhynchus sp. (ON)

Mollusca:

* Anodontoides ferrussacinea (ON)

* Lasmigona compressa (ON)

Branchiura:

Argulus catostomi (ON)

Copepoda:

Ergasilus versicolor (ON)

Ergasilus sp. (ON)

Lernaea cyprinacea (ON) 
Gasterosteus aculeatus Linnaeus

threespine stickleback

Monogenea:

Dactylogyrus sp. (BC)

Gyrodactyloidea gen. sp. (BC)

Gyrodactylus alexanderi (BC, NS)

Gyrodactylus avalonia (LB, NB, NF,

NT, QC)

Gyrodactylus canadensis (LB, NB,

NF, NS, NT, QC)

Gyrodactylus sp. (ON)

\section{Digenea:}

*Apatemon gracilis (BC)

* Apophallus brevis (QC)

Bunodera eucaliae (BC, ON)

Bunodera luciopercae (LB, NF)

Bunodera mediovitellata (BC)

Crepidostomum cooperi (NF)

Crepidostomum farionis (NF)

*Digenea gen. sp. (BC)

*Diplostomum scudderi (BC)

*Diplostomum spathaceum (BC, LB, NF, ON)

*Diplostomum sp. (BC, ON, QC)

*Posthodiplostomum minimum (BC)

*Tetracotyle sp. (BC, NF)

Cestoidea:

Bothriocephalidae gen. sp. (QC)

Bothriocephalus sp. (AB, NF)

Cestoidea gen. sp. (NF)

Cyathocephalus truncatus (BC)

*Diphyllobothrium dendriticum (NS)

*Diphyllobothrium ditremum (BC, CA, NS, QC)

*Diphyllobothrium sp. (BC, NT)

${ }^{*}$ Ligula intestinalis (NB, ON)
Proteocephalus filicollis (NF)

Proteocephalus pugetensis (BC, $\mathrm{ON})$

Proteocephalus sp. (AB, BC, LB, NF, NT)

*Schistocephalus solidus (AB, BC, LB, NB, NF, NT, ON, QC)

*Schistocephalus sp. (BC, QC)

Nematoda:

*Contracaecum sp. (LB, NF, NS)

*Cosmocephalus obvelatus (NS, ON)

*Eustrongylides sp. (BC, QC)

**Hysterothylacium sp. (ON)

* Paracuaria adunca (NB, NS, ON)

Philonema agubernaculum (QC)

Acanthocephala:

Echinorhynchus lateralis (LB, NF)

Echinorhynchus salmonis (CA, NF, ON)

Neoechinorhynchus rutili (BC, LB, NS, ON)

Neoechinorhynchus sp. (NT)

Annelida:

Dessorobdella phalera (EXP, ON)

Mollusca:

* Anodonta cataracta (NF)

* Anodonta cataracta cataracta (NS)

* Anodonta cataracta fragilis (NS)

* Anodonta implicata (NS)

*Anodonta sp. (NS)

* Elliptio complanata (NS)

*Lampsilis radiata radiata (NS)

*Unionidae gen. sp. (BC, ON)

Branchiura: 
Argulus stizostethi (EXP, NF, QC)

Copepoda:

Copepoda gen. sp. (BC)

Ergasilus auritus (BC, LB, NF)

Ergasilus nerkae (BC)

Ergasilus turgidus $(\mathrm{BC})$

Ergasilus sp. (ON)

Salmincola sp. (BC)

Therestina gasterostei (BC, LB, NF,

QC)

Pungitius pungitius (Linnaeus) ninespine stickleback

Monogenea:

Gyrodactylus avalonia (LB, NB, NF, NS, ON, QC)

Gyrodactylus canadensis (LB, NB,

NF, NS, NT, QC)

Gyrodactylus eucaliae (ON)

Gyrodactylus stephanus (NF)

Gyrodactylus sp. (AB, NT, ON)

Digenea:

*Apatemon gracilis (AB)

Bunodera eucaliae (ON)

Bunodera luciopercae (AB, NF)

*Diplostomum spathaceum (AB, NF, NT, ON)

*Ichthyocotylurus erraticus (AB)

*Tetracotyle sp. (ON)

Cestoidea:

Bothriocephalidae gen. sp. (QC)

*Diphyllobothrium dendriticum (NS, NT, QC)
*Diphyllobothrium ditremum (NS, NT)

*Diphyllobothrium sp. (LB, NF)

Eubothrium sp. (AB)

Proteocephalus filicollis (AB)

Proteocephalus sp. (LB, NF, NT, ON)

*Schistocephalus pungitii (NS)

*Schistocephalus solidus (AB, NS, NT, ON, QC)

*Schistocephalus sp. (MB, NB, NT)

*Triaenophorus nodulosus (AB)

Nematoda:

*Contracaecum sp. (MB, NS)

*Cosmocephalus obvelatus (NS)

* Paracuaria adunca (NS)

${ }^{*}$ Raphidascaris acus (AB)

Acanthocephala:

Acanthocephalus dirus (ON)

Echinorhynchus lateralis (NF)

Echinorhynchus salmonis (AB)

Leptorhynchoides thecatus (ON)

Neoechinorhynchus pungitius (ON)

Neoechinorhynchus rutili (BC, MB,

$\mathrm{NF}, \mathrm{NT}, \mathrm{ON}$ )

Neoechinorhynchus venustus (LB, QC)

Pomphorhynchus bulbocolli (AB)

Mollusca:

*Anodonta sp. (NS)

*Unionidae gen. sp. (ON)

Copepoda:

Ergasilus auritus $(\mathrm{AB})$

Ergasilus sp. (MB, ON)

Therestina gasterostei (LB, NF, NS,

QC) 


\section{ORDER SCORPAENIFORMES}

FAMILY COTTIDAE - sculpins

Cottus cognatus Richardson slimy sculpin

Monogenea:

Dactylogyrus buddi (ON, YT)

Gyrodactyloidea gen. sp. (BC)

Gyrodactylus bairdi (ON, YT)

Digenea:

Crepidostomum cooperi (LB)

Crepidostomum farionis (BC)

Crepidostomum metoecus (YT)

Crepidostomum sp. (ON)

Digenea gen. sp. (BC, MB)

*Diplostomum spathaceum (YT)

*Diplostomum sp. (BC)

*Heterophyidae gen. sp. (YT)

*Neascus sp. (YT)

*Tetracotyle sp. (BC, ON)

Cestoidea:

*Ligula intestinalis (BC)

Proteoc sphalus longicollis (YT)

*Schistocephalus solidus (MB, ON, YT)

*Triaenophorus nodulosus (MB, YT)

*Triaenophorus stizostedionis (MB)

Nematoda:

*Eustrongylides sp. (BC)

Haplonema sp. (BC)

*Nematoda gen. sp. (AB)
* Raphidascaris acus (YT)

Rhabdochona cotti (BC, ON)

Rhabdochona sp. (ON)

Spinitectus gracilis (ON)

Acanthocephala:

Acanthocephala dirus (ON)

Neoechinorhynchus rutili (YT)

Neoechinorhynchus tumidus (YT)

Cottus ricei (Nelson) spoonhead sculpin

Monogenea:

Dactylogyrus buddi (ON)

Digenea:

*Diplostomum spathaceum (ON)

*Diplostomum sp. (ON)

*Tetracotyle sp. (ON)

Cestoidea:

Cyathocephalus truncatus (ON)

Proteocephalus pearsei (ON)

Acanthocephala:

Acanthocephalus dirus (ON)

Neoechinorhynchus rutili (ON)

Mollusca:

*Unionidae gen. sp. (ON)

Copepoda:

Ergasilus sp. (ON) 
Cottus sp.

Cestoidea:

*Schistocephalus solidus (BC, QC)

Nematoda:

*Cosmocephalus obvelatus (ON)

* Paracuaria adunca (ON)

Rhabdochona canadensis (AB)

Myoxocephalus thompsoni (Girard) deepwater sculpin

Digenea:

*Diplostomum sp. (ON)

Cestoidea:

Bothriocephalus sp. (ON)

Cyathocephalus truncatus $(\mathrm{ON})$

Nematoda:

Cystidicola stigmatura (ON)

*Spirurinae gen. sp. (ON)

Acanthocephala:

Echinorhynchus salmonis (ON)

ORDER PERCIFORMES

FAMILY PERCIDAE - perches

Etheostoma exile (Girard) Iowa darter
Monogenea:

Aethycteron sp. (ON)

Dactylogyrus sp. (ON)

Gyrodactylus etheostomae (ON)

Gyrodactylus sp. (ON)

Urocleidus adspectus (ON)

Urocleidus sp. (ON)

Digenea:

Allocreadium sp. (ON)

Alloglossidium geminum (ON)

*Apophallus brevis (ON)

Azygia angusticauda (ON)

*Clinostomum complanatum (ON)

Crepidostomum cooperi (ON)

Crepidostomum isostomum (ON)

*Diplostomum spathaceum (ON)

*Diplostomum sp. (ON)

${ }^{*}$ Neascus sp. (ON)

Plagioporus sp. (ON)

*Posthodiplostomum minimum (ON)

*Strigeidae gen. sp. (ON)

*Tetracotyle sp. (ON)

*Uvulifer ambloplitis (ON)

Cestoidea:

Bothriocephalus cuspidatus (ON)

Bothriocephalus formosus (ON)

Corallobothrium sp. (ON)

*Hymenolepis sp. (ON)

Nematoda:

Camallanus oxycephalus (ON)

*Contracaecum sp. (ON)

Hysterothylacium brachyurum (ON)

**Nematoda gen. sp. (ON)

* Raphidascaris acus (ON)

Rhabdochona cascadilla (ON) 
Rhabdochona sp. (ON)

Acanthocephala:

Echinorhynchus sp. (ON)

Leptorhynchoides thecatus (ON)

Annelida:

Illinobdella sp.

Myzobdella sp. (ON)

Mollusca:

*Anodontoides ferrussacinea (ON)

Branchiura:

Argulus catostomi (ON)

Perca flavescens (Mitchill) yellow perch

Monogenea:

Cleidodiscus sp. (ON)

Gyrodactylidae gen. sp. (ON)

Gyrodactyloidea gen. sp. (ON)

Gyrodactylus eucaliae (ON)

Gyrodactylus freemani (ON)

Gyrodactylus sp. (ON)

Monoycnea gen. sp. (AB)

Onchocleidus chautauquensis (ON)

Tetraonchus monenteron (LB)

Urocleidus aculeatus (MB, ON)

Urocleidus adspectus (AB, EXP, MB, $\mathrm{NB}, \mathrm{ON}$ )

Digenea:

Alloglossidium geminum (ON)

*Apatemon gracilis (AB)
*Apophallus brevis (EXP, MB, NB, $\mathrm{NF}, \mathrm{ON}, \mathrm{QC}, \mathrm{SK})$

*Apophallus verustus $(\mathrm{ON}, \mathrm{QC})$

*Apophallus sp. (ON)

Azygia angusticauda (ON, QC)

Azygia longa (MB, ON)

Bucephalus elegans (ON)

Bunodera eucaliae (ON)

Bunodera luciopercae (AB, ON, QC)

Bunodera sacculata (MB, ON, QC)

Bunodera sp. (QC)

*Caecincola sp. (MB)

* *Centrovarium lobotes (ON)

*Clinostomum complanatum (MB, ON, QC)

*Clinostomum sp. (MB, NB)

*Crassiphialia bulboglossa (ON, QC)

Crepidostomum cooperi (MB, ON, QC)

Crepidostomum cornutum (ON)

Crepidostomum farionis (MB, ON)

Crepidostomum isostomum ( $\mathrm{AB}, \mathrm{QC})$

Crepidostomum sp. (ON)

Crepidostomum funduli (MB)

Cryptogonimus chili (ON)

**Digenea gen. sp. (MB, ON)

*Diplostomum adamsi (AB, ON)

*Diplostomum scudderi (ON)

*Diplostomum spathaceum (ON)

*Diplostomum spathaceum huronense (ON)

*Diplostomum spathaceum indistinctum $(\mathrm{ON})$

*Diplostomum sp. (AB, MB, ON)

*Ichthyocotylurus erraticus (ON)

*Ichthyocotylurus pileatus (AB, MB, ON)

Leuceruthrus micropteri (ON)

Lissorchis kritskyi (MB)

${ }^{*}$ Metorchis conjunctus (MB)

Microphallidae gen. sp. (ON)

Microphallus opacus (ON, QC) 
*Neascus pyriformis $(\mathrm{ON})$

*Neascus sp. (AB, BC, MB, ON)

*Ornithodiplostomum ptychocheilus (MB)

Phyllodistomum superbum (ON, QC)

Phyllodistomum sp. (ON)

*Posthodiplostomum minimum (ON)

*Posthodiplostomum minimum centrarchi (ON)

Prosorhynchoides pusilla (MB)

Ptychogonimus fontanus (QC)

*Rhipidocotyle papillosa (ON)

Rhipidocotyle papillosa (MB)

Sanguinicola occidentalis (ON)

*Strigeidae gen. sp. (MB, NB, ON)

*Tetracotyle sp. (AB, MB, ON)

*Tylodelphys scheuringi (AB, ON)

*Uvulifer ambloplitis (ON)

Cestoidea:

Bothriocephalus cuspidatus (AB, $\mathrm{HBD}, \mathrm{MB}, \mathrm{ON}$ )

*Bothriocephalus sp. (AB, MB, ON)

*Cestoidea gen. sp. (MB, QC)

Corallobothrium sp. (ON)

Cyathocephalus truncatus (NT, ON, QC)

*Diphyllobothrium latum (AB, MB, QC)

*Diphyllobothrium sp. (AB, QC)

Eubothrium sp. (AB)

${ }^{*}$ Ligula intestinalis $(\mathrm{AB}, \mathrm{BC}, \mathrm{MB}$, $\mathrm{NB}, \mathrm{ON}, \mathrm{QC}, \mathrm{SK}$ )

${ }^{*}$ Ligula sp. (AB)

**Proteocephalus ambloplitis (ON)

Proteocephalus pearsei (MB, ON)

Proteocephalus pinguis (ON, QC)

*Proteocephalus stizostethi (ON)

*Proteocephalus sp. (AB)

Proteocephalus sp. (AB, BC, LB, MB, NT, ON, QC)
- *Schistocephalus solidus (MB, ON)

*Schistocephalus sp. (AB)

*Triaenophorus crassus (HBD)

*Triaenophorus nodulosus (AB, CA, $\mathrm{MB}, \mathrm{ON}$ )

*Triaenophorus sp. (HBD, MB, ON)

Nematoda:

Camallamus oxycephalus (ON)

Camallanus sp. (ON)

*Contracaecum spiculigerum (AB, BC, ON)

Contracaecum sp. (MB, ON, QC)

Cucullanellus cotylophora (ON, QC)

Cucullamus sp. (ON)

*Eustrongylides tubifex (ON)

*Eustrongylides sp. (ON)

**Hysterothylacium brachyurum (ON)

**Nematoda gen. sp. (AB, MB, ON)

**Philometra cylindracea $(\mathrm{ON})$

${ }^{*}$ Raphidascaris acus (AB, BC, EXP, $\mathrm{MB}, \mathrm{ON}$ )

Raphidascaris acus (AB, ON, QC)

**Raphidascaris sp. (MB, ON, QC)

Rhabdochona cascadilla (ON)

Rhabdochona ovifilamenta (ON)

Rhabdochona sp. (ON)

Spinitectus carolini (ON)

Spinitectus gracilis (MB, ON)

Spinitectus sp. (MB, ON)

*Spiroxys contortus (ON)

*Spiroxys sp. (ON)

Acanthocephala:

Acanthocephala gen. sp. (ON)

Acanthocephala dirus (ON)

Echinorhynchus lateralis (QC)

Echinorhynchus salmonis (ON)

Leptorhynchoides thecatus ( $\mathrm{AB}, \mathrm{ON})$ 
Neoechinorhynchus crassus (MB)

Neoechinorhynchus cylindratus (MB, ON)

Neoechinorhynchus pungitius (ON)

Neoechinorhynchus rutili (MB, ON)

Neoechinorhynchus tenellus (ON)

Neoechinorhynchus venustus (ON)

Neoechinorhynchus sp. (ON)

Pomphorhynchus bulbocolli (AB, $\mathrm{MB}, \mathrm{ON}$ )

Annelida:

Actinobdella sp. (QC)

Desserobdella phalera (EXP, ON)

Illinobdella sp. (ON, QC)

Myzobdella lugubris (MB, ON, SK)

Myzobdella sp. (ON, QC)

Piscicola geometra (QC, SK)

Piscicola punctata (ON)

Piscicola sp. (ON)

Placobdella montifera (MB)

Placobdella parasitica (ON)

Mollusca:

*Anodonta grandis simpsonia (AB)

*Anodonta sp. (ON)

*Elliptio complanata (ON)

*Lampsilis radiata $(\mathrm{ON})$

*Lampsilis sp. (ON)

*Unionidae gen. sp. (AB, MB, ON)

Brachiura:

Argulus stizostethi (NS, ON)

Copepoda:

Achtheres pimelodi (ON)

Ergasilus caeruleus (AB, $\mathrm{ON})$

Ergasilus centrarchidarum $(\mathrm{ON})$
Ergasilus luciopercarum (MB, ON)

Ergasilus sp. (MB)

Arachnoidea:

*Hydrachna sp. (ON)

Percina caprodes (Rafinesque) logperch

Monogenea:

Aethycteron malleus (ON)

Gyrodactyloidea gen. sp. (ON)

Gyrodactylus sp. (ON)

Digenea:

Allopodocotyle boleosomi (ON)

Centrovarium lobotes (MB)

*Clinostomum complanatum (ON)

Crepidostomum isostomum (ON)

*Diplostomum sp. (MB, ON, SK)

Leuceruthrus micropteri (ON)

*Neascus sp. (ON)

*Posthodiplostomum cuticola (ON)

*Posthodiplostomum minimum (ON)

*Strigeidae gen. sp. (ON)

*Tetracotyle sp. (ON)

*Tylodelphys scheuringi (ON)

Cestoidea:

Bothriocephalus cuspidatus (ON)

Bothriocephalus formosus (ON)

Proteocephalus pearsei (ON)

**Proteocephalus stizostethi $(\mathrm{ON})$

Nematoda:

Camallamus oxycephalus (ON) 
*Contracaecum sp. (ON)

**Nematoda gen. sp. (ON)

*Raphidascaris sp. (MB)

Spinitectus gracilis (MB, ON)

Acanthocephala:

Echinorhynchus sp. (ON)

Leptorhynchoides thecatus (ON)

Pomphorhynchus bulbocolli (MB, ON)

Annelida:

Myzobdella lugubris (ON)

Piscicola punctata (ON)

Piscicolaria reducta (ON)

Copepoda:

Ergasilus caeruleus (ON)

Stizostedion canadense (Smith) sauger

Monogenea:

Urocleidus aculeatus (ON)

Digenea:

Centrovarium lobotes (ON)

*Clinostomum complanatum (ON)

Crepidostomum cooperi (MB)

Creptotrema funduli (MB)

*Diplostomum sp. (ON)

*Ichthyocotylurus platycephalus (ON)

${ }^{*}$ Neascus sp. (ON)

Phyllodistomum superbum (ON)

Prosorhynchoides pusilla (ON)

*Tetracotyle sp. (ON)
Cestoidea:

Bothriocephalus claviceps (ON)

Bothriocephalus cuspidatus (HBD, $\mathrm{MB}, \mathrm{ON}$ )

Cestoidea gen. sp. (MB)

*Diphyllobothrium latum (MB, QC)

*Proteocephalus ambloplitis (ON)

Proteocephalus luciopercae (MB, SK)

Proteocephalus stizostethi (ON)

*Triaenophorus crassus (AB)

Triaenophorus crassus (MB)

*Triaenophorus nodulosus (ON)

**Triaenophorus sp. (ON)

Nematoda:

Camallanus oxycephalus (ON)

*Eustrongylides sp. (ON)

**Hysterothylacium brachyurum (ON)

*Raphidascaris sp. (MB)

Acanthocephala:

Acanthocephala gen. sp. (ON)

Echinorhynchus salmonis (ON)

Neoechinorhynchus cylindratus (ON)

Neoechinorhynchus tenellus (ON)

Neoechinorhynchus sp. (ON)

Annelida:

Myzobdella lugubris (ON)

Piscicola punctata (ON)

Moilusca:

*Unionidae gen. sp. (ON)

Branchiure: 
Argulus appendiculosus (ON)

Copepoda:

Ergasilus caeruleus (ON)

Ergasilus centrarchidarum (ON)

Ergasilus luciopercarum (ON)

Stizostedion vitreum (Mitchill) walleye

Monogenea:

Cleidodiscus sp. (ON)

Gyrodactyloidea gen. sp. (ON)

Tetraonchus sp. (AB)

Urocleidus aculeatus (MB, ON)

Urocleidus adspectus (MB)

Digenea:

*Apophallus venustus (QC)

Azygia angusticauda (ON, QC)

Azygia longa (ON)

Azygia sp. (ON)

Bucephalus sp. (ON)

Bunodera sacculata (MB, ON)

*Centrovarium lobotes (MB, ON)

Centrovarium lobotes (MB, ON, QC)

*Clinostomum complanatum (MB, ON)

*Clinostomum sp. (MB)

* Crassiphialia bulboglossa (ON)

Crepidostomum cooperi (MB, QC)

Crepidostomum sp. (ON)

Creptotrema funduli (MB)

*Digenea gen. sp. (QC)

Digenea gen. sp. (ON)

*Diplostomum spathaceum (ON)

*Diplostomum sp. (MB, ON)

*Ichthyocotylurus pileatus (ON)

*Ichthyocotylurus platycephalus (ON)
*Neascus sp. (MB, ON)

*Ornithodiplostomum ptychocheilus

(MB)

Phyllodistomum superbum (ON)

*Posthodiplostomum minimum (ON)

Prosorhynchoides pusilla (MB, ON, QC)

Rhipidocotyle papillosa (MB)

Sanguinicola occidentalis (ON)

*Tetracotyle sp. (AB, ON)

*Tylodelphys scheuringi (ON)

*Uvulifer ambloplitis (ON)

Cestoidea:

Bothriocephalus cuspidatus (AB, HBD, MB, NT, ON, QC, SK)

Bothriocephalus sp. (AB, MB)

Cestoidea gen. sp. (AB, MB, QC)

Cyathocephalus sp. (AB)

*Diphyllobothriidae gen. sp. (MB)

*Diphyllobothrium latum (AB, MB, NT, ON, QC, SK)

*Diphyllobothrium sp. (AB, MB, NT)

Eubothrium sp. (MB)

*Proteocephalus ambloplitis (ON)

Proteocephalus luciopercae (MB, ON, SK)

Proteocephalus pearsei (ON)

Proteocephalus pinguis ( $\mathrm{AB}, \mathrm{ON})$

Proteocephalus stizostethi (ON, QC)

Proteocephalus sp. (AB, BC, LB, MB, NT, ON, QC, SK)

**Triaenophorus crassus (MB, ON)

*Triaenophorus nodulosus (MB, ON)

Triaenophorus nodulosus (AB, MB, ON)

Triaenophorus stizostedionis (AB, $\mathrm{MB}, \mathrm{NT}, \mathrm{ON}, \mathrm{SK}$ )

*Triaenophorus sp. (MB, ON)

Triaenophorus sp. (AB, MB, NT, $\mathrm{ON}$ ) 
Nematoda:

Camallanus oxycephalus (ON)

*Contracaecum sp. (QC)

Cucullamus cotylophora (ON)

*Eustrongylides tubifex (ON)

*Eustrongylides sp. (ON)

**Hysterothylacium brachyurum (ON)

*Nematoda gen. sp. (AB, MB, ON)

Philonema cylindracea (ON)

*Raphidascaris acus (MB)

Raphidascaris acus (MB, ON, QC)

*Raphidascaris sp. (MB, ON)

Rhabdochona canadensis (AB)

Spinitectus gracilis (ON)

Spinitectus sp. (MB, ON)

Acanthocephala:

Acanthocephala gen. sp. (ON)

Echinorhynchus salmonis (AB, ON)

Echinorhynchus sp. (ON)

Leptorhynchoides thecatus (ON)

Neoechinorhynchus crassus (MB)

Neoechinorhynchus cylindratus $(\mathrm{MB}$, ON)

Neoechinorhynchus rutili (ON)

Neoechinorhynchus strigosus (MB)

Neoechinorhynchus tenellus (MB, ON)

Neoechinorhynchus sp. (MB)

Pomphorhynchus sp. (MB)

Annelida:

Desserobdella phalera (EXP, ON)

Macrobdella decora (SK)

Myzobdella lugubris (AB, MB, ON)

Percymoorensis marmorata (SK)

Piscicola geometra (SK)

Piscicola punctata (ON, SK)

Piscicola sp. (ON)

Placobdella montifera (MB)
Mollusca:

*Unionidae gen. sp. (MB, ON)

Branchiura:

Argulus appendiculosus (MB, ON)

Argulus stizostethi (MB, ON, SK)

Argulus versicolor (ON)

Copepoda:

Copepoda gen. sp. (ON)

Ergasilus caeruleus (ON)

Ergasilus centrarchidarum (ON)

Ergasilus luciopercarum (MB, ON, QC)

Ergasilus sp. (MB) 
Acanthocephala

**Acanthocephala gen. sp.

Acanthocephala dirus

Acanthocephala jacksoni (see:

Acanthocephalus dirus)

Acanthocephalus lateralis (see:

Echinorhynchus lateralis)

Acanthocephalis sp.

Acarina

Acetodextra amiuri

Achtheres ambloplitis (see: Achtheres

pimelodi)

Achtheres coregoni (see: Salmincola extensus)

Achtheres corpulentus (see: Salmincola corpulentus)

Achtheres micropteri (see: Achtheres

corpulentus)

Achtheres pimelodi

Achtheres sp.

Acolpenteron catostomi

Actinobdella inequianmulata

Actinobdella sp.

Actinobdella triannulata (see: Actinobdella inequiannulata)

Acuariidae

Acuariodea

Adenophorea

Aethycteron malleus

Aethycteron sp.

Allobunodera mediovitellata (see: Bunodera mediovitellata)

Allobunodera sacculata (see: Bunodera sacculata)

Allocreadiata

Allocreadiidae

Allocreadiidae gen. sp.

Allocreadioidea

Allocreadium boleosomi (see:

Allopodocotyle boleosomi)

Allocreadium isoporum (see: Allocreadium lobatum) 
Allocreadium lobatum

Alloglossidium corti

Alloglossidium geminum

Allopodocotyle boleosomi

Amphicotylidae

Amphicotyloidea

Amphimerus elongatus

*Amphimerus pseudofelineus

Anallocreadium armatum (see:

Homalometron armatum)

Anallocreadium pearsei (see:

Homalometron armatum)

Ancyracanthus serratus (see: Cystidicola serrata)

Ancyrocepha.inae

Animalia

Anisakidae

*Anisakidae gen. sp.

Annelida

* Anodonta cataracta

Anodonta cataracta cataracta (see:

Anodonta cataracta)

Anodonta cataracta fragilis (see: Anodonta cataracta)

*Anodonta grandis simpsonia

*Anodonta implicata

*Anodonta sp.

*Anodontoides ferrussacinea

Anonchohaptor anomalum

Anonchohaptor muelleri

Anonchohaptor sp.

*Apatemon gracilis

Apatemon gracilis pellucidus (see:

Apatemon gracilis)

*Apophallus brevis

*Apophallus imperator

Apophallus itascensis (see: Apophallus brevis)

*Apophallus venustus

*Apophallus sp.

Arachnoidea

Argulidae
Arguloidea

Argulus appendiculosus

*Argulus biramosus

Argulus canadensis (see: Argulus stizostethi)

Argulus catostomi

Argulus sp.

Argulus stizostethi

Argulus versicolor

Arthropoda

Ascaridida

Ascaridoidea

Azygia acuminata (see: Azygia longa)

Azygia angusticauda

Azygia longa

Azygia lucii (see: Azygia longa)

Azygia sp.

Azygiidae

Biacetabulum biloculoides

Biacetabulum infrequens

Biacetabulum sp.

Bivalvia

Bothrimonas intermedius (see: Bothrimonas siurionis)

Bothrimonas sturionis

Bothrimonidae

Bothriocephalata

Bothriocephalidae

Bothriocephalidae gen. sp.

Bothriocephalus acheilognathi

Bothriocephalus claviceps

Bothriocephalus cuspidatus

Bothriocephalus cuspidatus cuspidatus (see: Bothriocephalus cuspidatus)

Bothriocephalus cuspidatus hiodontos (see:

Bothriocephalus cuspidatus)

Bothriocephalus cuspidatus luciopercae

(see: Bothriocephalus cuspidatus)

Bothriocephalus fluviatilis (see:

Bothriocephalus acheilognathi)

Bothriocephalus formosus

Bothriocephalus gowkongensis (see: 
Bothriocephalus acheilognathi)

Bothriocephalus opsariichthydis (see:

Bothriocephalus acheilognathi)

Bothriocephalus sp.

Brachylaemoidea

Branchiura

Bucephalidae

Bucephalopsis ozakii (see:

Prosorhynchoides ozakii)

Bucephalis elegans

Bucephalis pusilla (see Prosorhynchoides pusilla)

Bucephalopsis pusilla (see:

Prosorhynchoides pusilla)

Bucephalus sp.

Bulbodacnitis alpinus (see: Truttaedacnitis truttae)

Bulbodacnitis truttae (see: Truttaedacnitis truttae)

Bunodera eucaliae

Bunodera inconstans (see: Culaeatrema inconstans)

Bunodera luciopercae

Bunodera mediovitellata

Bunodera sacculata

Bunodera sp.

Bunoderina eucaliae (see: Bunodera eucaliae)

Bunoderina sacculata (see: Bunodera sacculata)

*Caecincola sp.

Camallanidae

Camallanina

Camallanoidea

Camallanus ancylodirus

* *amallanus oxycephalus

Camallamus sp.

Capillaria (Capillaroides) catenata

Capillaria catostomi (see Pseudocapillaria

(Pseudocapillaria) tomentosa

Capillaria salvelini (see Pseudocapillaria
(Ichthyocapillaria) salvelini)

Capillariidae

Capillariidae gen. sp.

Capingentidae

Caryophyllaeidae

Caryophyllidea

Caryophyllidea gen. sp.

*Centrovarium lobotes

Centrovarium lobotes

Cestoda

Cestoidea

*Cestoidea gen. sp.

Cestoidea gen. sp.

Cleidodiscus aculeatus (see Urocleidus aculeatus)

Cleidodiscus adspectus (see Urocleidus adspectus)

Cleidodiscus baldwini (see Urocleidus baldwini)

Cleidodiscus brachus

Cleidodiscus malleus (see: Ligictalurides pricei)

Cleidodiscus sp.

Clepsine phalera (see: Desserobdella phalera)

Clinosomoidea

*Clinostomum complanatum

Clinostomum marginatum (see:

Clinostomum complanatum)

*Clinostomum sp.

Cnidaria

Contracaecum aduncum (see:

Hysterothylacium aduncum)

Contracaecum brachyurum (see:

Hysterothylacium brachyurum)

Contracaeum clavatum (see:

Hysterothylacium aduncum)

Contracaecum gadi (see: Hysterothylacium aduncum)

* Contracaecum spiculigerum

* Contracaecum sp.

Copepoda 
Copepoda gen. sp.

Corallobothrium fimbriatum

Corallobothrium sp.

Coregonicola orientalis

Coregonicola sp.

*Cosmocephalus obvelatus

Cottocomephoronema hamulatum (see: Haplonema hamulatum)

Cottocomephoronema problematicum (see: Haplonema hamulatum)

Cotylurus communis (see: Ichthyocotylurus platycephalus)

Cotylurus erraticus (see: Ichthyocotylurus erraticus)

*Crassiphialia bulboglossa

Crepidostomum ambloplitis (see:

Crepidostomum cooperi)

Crepidostomum auriculatum

Crepidostomum cooperi

Crepidostomum cornutum

Crepidostomum farionis

Crepidostomum fausti (see: Crepidostomum cooperi)

Crepidostomum hiodontis (see:

Crepidostomum illinoiense)

Crepidostomum illinoiense)

Crepidostomum isostomum

Crepidostomum lintoni (see:

Crepidostomum auriculatum)

Crepidostomum meteocus

Crepidostomum percopsisi

Crepidostomum sp.

Creptotrema funduli

Crustacea

Cryptogonimidae

*Cryptogonimus chili

Cryptogonimus chili

Cucullamus cotylophora

Cucullanidae

Cucullanus clitellarius (see: Truttaedacnitis clitellarius)

Cucullanus sp.
Culaeatrema inconstans

Cuninidae

Cyathocephalidae

Cyathocephalus sp.

Cyathocephalus truncatus

Cyclophyllidea

Cyclopoidea

Cystidicola canadensis (see: Cystidicola farionis)

Cystidicola cristivomeri (see: Cystidicola stigmatura)

Cystidicola farionis

Cystidicola serratus

Cystidicola sp.

Cystidicola stigmatura

Cystidicola walkeri (see: Salvelinema walkeri)

Cystidicolidae

Cystidicoloides ephemeridarum (see:

Sterliadochona ephemeridarum)

Cystidicoloides harwoodi (see:

Sterliadochona ephemeridarum)

Cystidicoloides tenuissima (see:

Sterliadochona ephemeridarum)

Cystobranchus mammilatus

Cystidicola meyeri

Cystobranchus verrilli

Dacnitoides cotylophora (see: Cucullanellus cotylophora)

Dactylogyridae

Dactylogyridae gen. sp.

Dactylogyridea

Dactylogyrinae

Dactylogyrinea

Dactylogyrus albertensis

Dactylogyrus banghami

Dactylogyrus bifurcatus

Dactylogyrus buddi

Dactylogyrus bychowskyi

Dactylogyrus chrosomi

Dactylogyrus columbiensis 
Dactylogyrus duquesni

Dactylogyrus eos

Dactylogyrus eucalius

Dactylogyrus hankinsoni

Dactylagyrus mollis

Dactylogyrus mylocheilus

Dacytlogyrus ptychocheilus

Dactylogyrus richardsoni

Dactylogyrus sp.

Dactylogyrus tridactylus

Dactylogyrus urus

Dactylogyrus vancleavei

Deropristidae

Deropristis hispida

Desserobde!la phalera

Dibothriocephalus latus (see:

Diphyllobothrium latum)

Dichelyne cotylophora (see: Cucullanellus cotylophora)

Diclybothriidae

Diclybothriidea

Diclybothrium armatum

Diclybothrium hammulatum

Digenea

*Digenea gen. sp.

Digenea gen. sp.

Dilepididae

Dioctophymatidae

Dioctophymatoidea

Diphyllobothriata

Diphyllobothriidae

*Diphyllobothriidae gen. sp.

Diphyllobothrium cordiceps (see:

Diphyllobothrium dendriticum)

Diphyllobothrium dendriticum

*Diphyllobothrium ditremum

*Diphyllobothrium laruei

*Diphyllobothrium latum

Diphyllobothrium osmeri (see:

Diphyllobothrium ditremum)

*Diphyllobothrium sp.

Diplobothrium hammulatum (see:
Diclybothrium hammulatum)

Diplocotyle olrikii (see: Bothrimonas sturionis)

Diplostomidae

Diplostominae

Diplostomum flexicaudum (see:

Diplostomum spathaceum indistictum)

Diplostomum huronense (see: Diplostomum spathaceum huronense)

Diplostomulum spathaceum (see:

Diplostomum spathaceum)

Diplostomulum sp. (see: Diplostomum sp.)

* Diplostomum adamsi

*Diplostomum baeri bucculatum

Diplostomum baeri eucaliae (see:

Diplostomum scudderi)

Diplostomum flexicaudum (see:

Diplostomum spathaceum indistinctum)

Diplostomum huronense (see: Diplostomum spathaceum huronense)

Diplostomum parvula (see: Tetrocotyle sp.)

Diplostomum scheuringi (see: Tylodelphys scheuringi)

*Diplostomum scudderi

*Diplostomum spathaceum

* Diplostomum spathaceum huronense

* Diplostomum spathaceum indistinctum

*Diplostomum sp.

Discocotyle sagittata

Discocotyle salmonis (see: Discocotyle sagittata)

Discocotyle sp.

Discocotylidae

Discocotylinea

Distomum folium (see: Phyllodistomum folium)

Distomum isostomum (see: Allocreadium lobatum)

Distomum isoporum var. armatum (see: Homalometron armatum)

Distomum lobotes (see: Centrovarium lobotes) 
Distomum nodulosum (see: Bunodera luciopercae)

Distomum opacus (see: Microphallus opacus)

Dracunculoidea

Echinorhynchidae

Echinorhynchoidea

Echinorhynchus coregonus (see: Echinorhynchus salmonis)

Echinorhynchus gadi

Echinorhynchus lateralis

Echinorhynchus leidyi

Echinorhynchus salmonis

Echinorhynchus salvelini (see:

Echinorhynchus leidyi)

Echinorhynchus sp.

Echinorhynchus thecatus (see:

Leptorhynchoides thecatus)

Echinorhynchus vancleavi (see:

Echinorhynchus gadi)

*Elliptio complanata

Enoplida

Entomostraca

Eoacanthocephala

Eorhynchus cylindratus (see:

Neoechinorhynchus cylindratus)

Ergasilidae

Ergasilus auritus

Ergasilus caeruleus

Ergasilus celestis

Ergasilus centrarchidarum

Ergasilus confusus (see: Ergasilus luciopercarum)

Ergasilus cyprinacea

Ergasilus elegans (see: Ergasilus versicolor)

Ergasilus elongatus

Ergasilus funduli (see: Ergasilus labracis)

Ergasilus labracis

Ergasilus lizae

Ergasilus luciopercarum

Ergasilus nerkae
Ergasilus osborni (see: Ergasilus celestis)

Ergasilus sp.

Ergasilus turgidus

Ergasilus versicolor

Eubothrium crassum

Eubothrium rugosum

Eubothrium salvelini

Eubothrium sp.

Eubothrium tulipai

Eucestoda

Eulamellibranchia

Eumetazoa

*Eustrongylides sp.

*Eustrongylides tubifex

Gasterostomum pusillum (see:

Prosorhychoides pusilla)

Glaridacris catostomi

Glaridacris confusus

Glaridacris intermedius (see: Glaridacris laruei)

Glaridacris laruei

Glaridacris sp.

Glaridacris terebrans

Glaridacris vogei

Glossidium geminum (see: Alloglossidium geminum)

Glossiphonidae

Glossiphonidae gen. sp.

Gnathobdellida

Gnathostomatidae

Gnathostomatoidea

Gorgoderidae

Gorgoderidae gen. sp.

Gorgoderoidea

Gyrodactylidae

Gyrodactylidae gen. sp.

Gyrodactyloidea gen. sp.

Gyrodactylus aldrichi

Gyrodactylus alexanderi

Gyrodactylus avalonia

Gyrodactylus aquilinus 
Gyrodactylus atratuli

Gyrodactylus bairdi

Gyrodactylus calliaratus

Gyrodactylus canadensis

Gyrodactylus cheloideus

Gyrodactylus colemanensis

Gyrodactylus commersoni

Gyrodactylus couesius

Gyrodactylus dechtiari

Gyrodactylus etheosomae

Gyrodactylus eucaliae

Gyrodactylus freemani

Gyrodactylus hoffmani

Gyrodactylus labradorius (see:

Gyrodactylus bairdi)

Gyrodactylus lacustris

Gyrodactylus lairdi (see: Gyrodactylus avalonia)

Gyrodactylus limi

Gyrodactylus lotae

Gyrodactylus lucii

Gyrodactylus margaritae

Gyrodactylus memorialis (see: Gyrodactylus avalonia)

Gyrodactylus perlucidus

Gyrodactylus plumbae

Gyrodactylus salmonis

Gyrodactylus spathulatus

Gyrodactylus sp.

Gyrodactylus stephanus

Gyrodactylus stunkardi

Gyrodactylus terranovae (see: Gyrodactylus avalonia)

Habronematoidea

\section{Haplonema hamulatum}

Haplonema sp.

Hemiuriformes

Hemiuroidea

Hapaticola bakeri (see: Pseudocapillaria

- (Pseudocapillaria) tomentosa)

Heterophyes sp.
Heterophyidae

${ }^{*}$ Heterophyidae gen. sp.

Hirudinea

Hirudinea gen. sp.

Hirudinidae gen. sp.

Hirudinidae

Homalometridae

Homalometron armatum

Hunterella nodulosa

*Hydrachna sp.

Hydrachnae

Hydrachnidae

Hydrozoa

Hymenolepididae

*Hymenolepis sp.

Hypocaryophallaeus paratarius

*Hysterothylacium aduncum

*Hysterothylacium brachyurum

Hysterothylacium brachyurum

${ }^{* *}$ Hysterothylacium sp.

Icelanonchohaptor fyviei

Icelanonchohaptor microcotyle

Ichthyobronema gnedini (see: Haplonema hamulatum)

*Ichthyocotylurus erraticus

*Ichthyocotylurus pileatus

*Ichthyocotylurus platycephalus

Ic'?thyotaenia laruei (see: Protocephalus laruei)

Illinobdella alba (see: Myzobdella lugubris)

Isoglaridacris bulbocirrus

Isoglaridacris calentinei

Khawia iowensis

*Lampsilis radiata radiata

Lampsilis radiata siliquoides (see: Lampsilis radiata radiata)

*Lampsilis sp.

* Lasmigonia compressa

Lebouria cooperi (see: Plagioporus cooperi) 
Lecithodendriidae

Lepocreadiiformes

Lepocreadioidea

**Leptorhynchoides thecatus

Leptorhynchoididae

Lernaea catostomi

Lernaea cyprinacea

Lernaeidae

Lernaeopoda arcturi (see: Salmincola edwardsii)

Lernaeopoda edwardsii (see: Salmincola edwardsii)

Lernaeopodidae

Leuceruthrinae

*Leuceruthrus sp.

Leuceruthrus sp.

Ligictaluridus posthon

Ligictaluridus pricei

${ }^{*}$ Ligula intestinalis

*Ligula sp.

Ligulidae

Lissorchiidae

Lissorchis attenuatus

Lissorchis crassicrurum

Lissorchis gullaris

Lissorchis kritskyi

Lissorchis simeri

Lissorchis sp.

Lissorchis transluscens

Lytocestidae

Macrobdella decora

Macroderoididae

Markewitschia aurita (see: Ergasilus auritus)

Mazocraeida

Mazocraeidae

Mazocraeoidea

Mazocraeoides sp.

Megastomum longa (see: Azygia longa)

Megalonia ictaluri

Metabronema sp.
Metabronema canadense (see:

Sterliadochona ephemeridarum)

Metabronema salvelini (see: Sterliadochona ephemeridarum)

Metechinorhynchus lateralis (see:

Echinorhynchus lateralis)

Metechinorhynchus leidyi (see:

Echinorhynchus leidyi)

Metechinorhynchus salmonis (see:

Echinorhynchus salmonis)

*Metorchis conjunctus

Microphallidae

*Microphallidae gen. sp.

Microphalloidea

Microphallus cpacus

Mimodistomum angusticaudum (see: Azygia angusticauda)

Mollibdella grandis

Mollusca

Monobothrium hunteri

Monobothrium sp.

Monodistomum amiuri (see: Acetodextra amiuri)

Monogenea

Monogenea gen. sp.

Myzobdella alba (see: Myzodella lugubris)

Myzodella lugubris

Myzobdella moorei (see: Myzobdella lugubris)

Myzobdella sp.

Narcomedusae

*Neascus pyriformis

*Neascus rhinichthysi

*Neascus sp.

Nemathelminthes

Nematoda

**Nematoda gen. sp.

Neodiscocotyle carpioditis

Neoechinorhynchidae

Neoechinorhynchus carpiodi

Neoechinorhynchus crassus 
Neoechinorhynchus cristatus (see:

Neoechinorhynchus venustus)

Neoechinorhynchus cylindratus

Neoechinorhynchus dictractus

Neoechinorhynchus notemigoni

Neoechinorhynchus pungitius

Neoechinorhynchus rutili

Neoechinorhynchus saginatus

Neoechinorhynchus salmonis

Neoechinorhynchus sp.

Neoechinorhynchus strigosus

Neoechinorhynchus tenellus

Neoechinorhynchus tumidus

Neoechinorhynchus venustus

Neorhynchus cylindratus (see:

Neoechinorhynchus cylindratus)

Neorhynchus tenellus (see:

Neoechinorhynchus tenellus)

Nipporhynchus trachuri (see:

Rhadinorhynchus trachuri)

Octomacridae

Octomacrum lanceatum

Octomacrum microconfibulum

Octomacrum semotili

Octomacrum sp.

Octospinifer macilentus

Onchocleidus chautauquensis

Opecoelidae

Opecoeloidea

Opisthorchiidae

Opisthorchiirormes

Opisthorchis pseudofelineus (see:

Amphimerus psudofelineus)

Opisthorchis sp.

Opisthorchoidea

*Ornithodiplostomum ptychocheilus

Paleacanthocephala

* Paracuaria adunca

Paradiclybothrium sp.

* Paradilepis simoni
*Paradilepis sp.

* Parametorchis complexus

Parametorchis manitobensis (see:

Parametorchis complexus)

Parametorchis novaeboracensis (see:

Parametorchis complexus)

Paurorhynchus hiodontos

Pellucidhaptor angularis

Pellucidhaptor catostomi

Pellucidhaptor eremitis

Pellucidhaptor eriensis

Pellucidhaptor micracanthus

Pellucidhaptor moxostomi

Pellucidhaptor nasalis

Pellucidhaptor pellucidhaptor

Pellucidhaptor sp.

Pellucidhaptor thelostea

Percymoorensis marmorata

Philometra kobuleji

Philometra nodulosa (see: Philometroides nodulosa)

**Philometra sp.

Philometridae

Philometroides huronensis

Philometroides nodulosa

*Philometroides sp.

Philonema agubernaculum

**Philonema cylindracea

**Philonema oncorhynchi

* Philonema sp.

Phyllobothriidae

Phyllobothrium caudatum

Phyllobothrium ketae (see: Phyllobothrium caudatum)

Phyllobothrium salmonis (see:

Phyllobothrium caudatum)

Phyllobothrium sp.

Phyllodistomum coregoni

Phyllodistomum folium

Phyllodistomum lachancei (see:

Phyllodistomum umblae)

Phyllodistomum limnosa (see: 
Phyllodistomum umblae)

Phyllodistomum lysteri

Phyllodistomum sp.

Phyllodistomum superbum

Phyllodistomum umblae

Pionae

Piscicola geometra

Piscicola milneri

Piscicola punctata

Piscicola salmositica

Piscicola sp.

Piscicolaria reducta

Piscicolidae

Placobdella montifera

Placobdella parasitica

Placobdella sp.

Plagiocirrus sp.

Plagioporus cooperi

Plagioporus serratus

Plagioporus shawi

Plagioporus sinitsini

Plagioporus sp.

Plagiorchiata

Plagiorchiiformes

Plagiorchis geminus (see: Alloglossidium geminum)

Plagiorchoidea

Platyhelminthes

Pleurogenes sp.

Pliovitellaria wisconsinensis

Podocotyle boleosomi (see: Allopodocotyle boleosomi)

Poecilostomatoidea

Polyonchoina

Polypodium hydriforme

Polypodium sp.

Pomphorhynchidae

**Pomphorhynchus bulbocolli

Pomphorhynchus sp.

*Posthodiplostomum cuticola

*Posthodiplostomum minimum

*Posthodiplostomum minimum centrarchi
*Posthodiplostomum minimum minimum

Prosorhynchoides ozakii

Prosorhynchoides pusilla

Proteocephalida

Proteocephalidae

Prosteocephalidae gen. sp.

Proteocephalus ambloplitis

Proteocephalus arcticus (see:

Proteocephalus longicollis)

Proteocephalus coregoni

Proteocephalus exiguus (see:

Proteocephalus longicollis)

Proteocephalus filicollis

Proteocephalus fluviatilis

Proteocephalus laruei (see: Proteocephalus

longicollis)

Proteocephalus longicollis

Proteocephalus luciopercae

Proteocephalus micropteri (see:

Proteocephalus ambloplitis)

Proteocephalus neglectus (see:

Proteocephalus longicollis)

Proteocephalus parallacticus

Protocephalus pearsei

Proteocephalus pinguis

Proteocephalus pugetensis

Proteocephalus pusillus (see:

Proteocephalus longicollis)

Proteocephalus salmonidicola (see:

Proteocephalus longicollis)

Proteocephalus singularis

**Proteocepher'is sp.

*Proteocephalus stizostethi

Proteocephalus torulosus

Proteocephalus wickliffi (see:

Proteocephalis longicollis)

Pseudocapillaria (Ichthyocapillaria)

salvelini

Pseudocapillaria (Pseudocapillaria)

tomentosa

Pseudomurraytrema alabarrum

Pseudomurraytrema copulatum 
Pseudomurraytrema muelleri

Pseudomurraytrema sp.

Pseudomurraytrematinae

Pseudophyllidea

Ptychogonimus fontanus (see: Azygia angusticauda)

Quimperidae

*Raphidascaris acus

Raphidascaris acus

Raphidascaris alius (see: Raphidascaris acus)

Raphidascaris canadensis (see:

Raphidascaris acus)

Raphidascaris laurentiamus (see:

Raphidascaris acus)

** Raphidascaris sp.

Rhabdochona canadensis

Rhabdochona cascadilla

Rhabdochona catostomi

Rhabdochona corti

Rhabdochona decaturensis

Rhabdochona fortunowi (see: Rhabdochona ovifilamenta)

Rhabdochona kisutchi

Rhabdochona laurentiana (see:

Rhabdochona ovifilamenta)

Rhabdochona milleri

Rhabdochona ovifilamenta

Rhabdochona rotundicaudatum

Rhabdochona serrata (see: Cystidicola serrata)

Rhabdochona sp.

Rhabdochona zacconis

Rhabdochonidae

*Rhabdochonidae gen. sp.

Rhadinorhynchus trachuri

*Rhipidocotyle papillosa

Rhipidocotyle papillosa

*Rhipidocotyle sp.

Rhynchobdellida
Rowardleus pennensis

Salmincola bicauliculata (see: Salmincola californiensis)

Salmincola californiensis

Salmincola carpionis

Salmincola corpulentus

Salmincola edwardsii

Salmincola extensus

Salmincola exsanguinata (see: Salmincola edwardsii)

Salmincola extumescens (see: Salmincola corpulentus)

Salmincola giber (see: Salmincola carpionis)

Salmincola inermis (see: Salmincola corpulentus)

Salmincola lotae

Salmincola siscowet

Salmincola sp.

Salmincola thymalli

Salmincola wisconsinensis (see: Salmincola extensus)

Salvelinema salmonicola

Salvelinema walkeri

Sanguinicola occidentalis

Sanguinicola sp.

Sanguinicolidae

Sanguinicolidae gen. sp.

Schistocephalidae

*Schistocephalus pungitii

* Schistocephalus solidus

*Schistocephalus sp.

Schistosomatoidea

Secernentea

*Seuratinae gen. sp.

Seuratoidea

Siphonostomatoidea

Skrjabinocapillaria bakeri (see:

Pseudocapillaria (Pseudocapillari) tomentosa)

Skrjabinopsolus manteri 
Spartoides wardi

Spathebothriidea

Spinitectus acipenseri

Spinitectus carolini

**Spinitectus gracilis

Spinitectus sp.

*Spiroxys contortus

*Spiroxys sp.

Spirurida

*Spiruridae gen. sp.

Spirurina

*Spirurinae gen. sp.

Sterliadochona ephemeridarum

Sterliadochona sp.

Sterlidochona temuissima (see:

Sterliadochona ephemeridarum)

Strigeidae

*Strigeidae gen. sp.

Strigeiformes

Strigeoidea

Tanaorhamphus sp.

Tanaorhynchus sp. (see: Tanaorhamphus sp.)

Tenuisentidae

Tetracotyle diminuta (see: Ichthyocotylurus pileatus)

Tetracotyle intermedia (see:

Ichthyocotylurus erraticus)

Tetracotyle parvula (see: Tetracotyle sp.)

* Tetracotyle sp.

*Tetrameres sp.

Tetrameridae

Tetraonchidae

Tetraonchinea

Tetraonchus alaskensis

Tetraonchus borealis

Tetraonchus loftusi

Tetraonchus monenteron

Tetraonchus sp.

Tetraonchus variabilis

Tetraphyllidea
Tetraphyllidea gen. sp.

Thelazioidea

Therestina gasterostei

Thominx catenata (see: Capillaria (Capillaroidea) catenata)

Thynnascaris adunca (see: Hysterothylacium aduncum)

Thynnascaris brachyurum (see: Hysterothylacium brachyurum)

Trachylina

Trematoda

Trematoda gen. sp. (see: Digenea gen. sp.)

Triaenophoridae

*Triaenophorus crassus

Triaenophorus crassus

*Triaenophorus nodulosus

Triaenophorus nodulosus

Triaenophorus robustus (see: Triaenophorus crassus)

*Triaenophorus sp.

Triaenophorus sp.

*Triaenophorus stizostedionis

Triaenophorus stizostedionis

Triaenophorus tricuspidatus morpha megadontus (see: Triaenophorus crassus)

Triaenophorus tricuspidatus morpha microdentatus (see: Triaenophorus nodulosus)

Trichuroidea

Triganodistomum attenuatus (see:

Lissorchis attenuatus)

Trombidiformes

Truttaedacnitis clitellarius

Truttaedacnitis sp.

Truttaedacnitis truttae

*Tylodelphys scheuringi

Unionacea

*Unionicola sp.

Unionicolidae

Unionidae

*Unionidae gen. sp. 
Urocleidus aculeatus

\section{INDEX TO HOSTS}

Urocleidus adspectus

Urocleidus baldwini

Urocleidus brachus (see: Cleidodiscus

brachus)

Urocleidus malleus (see: Aethycteron malleus)

Urocleidus chautauquensis (see:

Onchocleidus chautauquensis)

Urocleidus sp.

*Uvulifer ambloplitis 
Acipenser fulvescens

Acipenseridae

Arctic lamprey

Arctic grayling

Brassy minnow

Brook charr

Brook stickleback

Brown trout

Bull charr

Bullhead catfishes

Burbot

Carpiodes cyprinus

Catostomidae

Catostomus catostomus

Catostomus commersoni

Catostomus macrocheilus

Catostomus platyrhynchus

Catostomus sp.

Cisco

Cods

Coho salmon

Coregonus artedii

Coregonus clupeaformis

Coregonus sp.

Coregonus zenithicus

Cottidae

Cottus cognatus

Cottus ricei

Cottus sp.

Couesius plumbeus

Culaea inconstans

Cuttroat trout

Cyprinidae

Deepwater sculpin

Dolly Varden

Emerald shiner

Esocidae

Esox lucius
Etheostoma exile

Fathead minnow

Finescale dace

Flathead chub

Gadidae

Gasterosteidae

Gasterosteus aculeatus

Goldeye

Hiodon alosoides

Hiodon tergisus

Hiodontidae

Hybognathus hankinsoni

Ictaluridae

Iowa darter

Lake charr

Lake chub

Lake herring

Lake sturgeon

Lake whitefish

Lampetra japonica

Lampreys

Largescale sucker

Logperch

Longnose dace

Longnose sucker

Lota lota

Margariscus margarita

Minnows

Mooneye

Mooneyes

Mountain sucker

Mountain whitefish

Moxostoma anisurum

Moxostoma macrolepidotum

Myoxocephalus thompsoni

Ninespine stickleback 
Northern redbelly dace

Northern pike

Northern squawfish

Notropis atherinoides

Notropis hudsonius

Noturus flavus

Oncorhynchus clarki

Oncorhynchus kisutch

Oncorhynchus mykiss

Pearl dace

Perca flavescens

Perches

Percidae

Percina caprodes

Percopsidae

Percopsis omiscomaycus

Petromyzontidae

Phoxinus eos

Phoxinus neogaeus

Pikes

Pimephales promelas

Platygobio gracilis

Prosopium coulteri

Prosopium cylindraceum

Prosopium williamsoni

Prosopium sp.

Ptychocheilus oregonensis

Pungitius pungitius

Pygmy whitefish

Quillback

Rainbow trout

Redside shine

Rhinichthys cataractae

Richardsonius balteatus

Round whitefish

Salmo trutta

Salmonidae
Salmonidae gen. sp.

Salvelinus confluentus

Salvelinus fontinalis

Salvelinus fontinalis X Salvelinus namaycush

Salvelinus malma

Salvelinus namaycush

Sculpins

Sauger

Shorthead redhorse

Shortjaw cisco

Silver redhorse

Slimy sculpin

Splake

Spoonhead sculpin

Spottail shiner

Sticklebacks

Stizostedion canadense

Stizostedion vitreum

Stonecats

Suckers

Threespine stickleback

Thymallus arcticus

Trout-perch

Trout-perches

Trouts

White sucker

Walleye

Yellow perch 
Adamson, M. L., Clease, D. F. and Margolis, L. 1992. Differentiation of Philonema spp. (Nematoda: Philometridae) in British Columbia salmonid fishes using DNA restriction fragment length differences. Canadian Journal of Fisheries \& Aquatic Sciences. 49: 1650-1656.

Albert, E. and Curtis, M.. 1988. Experimental depletion of a brook trout population to improve yield and decrease parasite abundance. Bulletin of the Aquaculture Association of Canada. 88-4: 22-24.

Albert, E. and Curtis, M.. 1991. Prevalence and abundance of helminth parasites in an intensely fished population of brook trout (Salvelinus fontinalis) at a small subarctic lake. Canauiian Journal of Zoology. 69: 691697.

Allen, J. A. and Wardle, R. A. 1934. Fluke disease in northern Manitoba sledge dogs. Canadian Journal of Research, Section D. 10: 404-408.

Amedjo, S. D. and Holmes, J. C. 1989. Color, size, and maturation of Pomphorhynchus bulbocolli (Acanthocephala) in white suckers, Catostomus commersoni. Journal of Parasitology. 75: 798-800.

Anderson, K., Ching, H. L. and Vik, R. 1987. A review of freshwater species of Diphyllobothrium with descriptions and distribution of $D$. dendriticum (Nitzsch, 1824) and $D$. ditremum (Creplin, 1825) from North America. Canadian Journal of Zoology. 65: 2216-2228.

Anderson, K. I. and Kennedy, C. R. 1983. Systematics of the genus Eubothrium Nybelin 
(Cestoda: Pseudophyllidea), with partial redescription of the species. Zoologica Scripta. 12: 95-105.

Anderson, D. M. 1993. Occurrence of Salmincola siscowet (Copepoda: Lernaeopodidae) on lake trout (Salvelinus namaycush) and lake trout backcross ( $S$. namaycush $\mathrm{x} S$. fontinalis) in Lake Huron. Canadian Journal of Zoology. 71: 2330-2333.

Anderson, R. C. and Wong, P. L. 1982. The transmission and development of Paracuaria adunca (Creplin, 1846) (Nematoda: Acuarioidea) of gulls. Canadian Journal of Zoology. 60: 3092-3104.

Anonymous. 1976. Commercial fisheries catch statistics for Alberta, 1942-1975. Alberta Department of Recreation, Parks \& Wildlife, Fish \& Wildlife Division. Fisheries Management Report. No. 22: 211 p.

Anonymous, 1978. Report on environmental studies for the McGregor diversion project. Volume 4 - Faunal transfer. Prepared for British Columbia Hydro and Power Authority, Vancouver, British Columbia by Reid, Crowther \& Partners, Ltd. vi + 143 p.

Anonymous. 1981. Kemano Completion Hydroelectric Development Baseline Studies. Volume 7. Fish diseases and parasites. Prepared for Aluminum Company of Canada, Vancouver, British Columbia by Environcon Ltd., Vancouver, British Columbia. 179 p.

Anonymous. 1984. Environmental studies associated with the proposed Kemano Completion Hydroelectric Development. Volume 7. Fish diseases and parasites, 1979 initial baseline environmental studies. Section
A, B and C. Prepared for Aluminum Company of Canada Ltd., Vancouver, British Columbia by Environcon Ltd., Vancouver, British Columbia. $179 \mathrm{p}$.

Anthony, D. D. 1967. Taxonomy and ecology of Diphyllobothrium in Alberta and British Columbia. Ph. D. Dissertation. Department of Zoology, University of Alberta, Edmonton, Alberta.

Anthony, D. D. 1974. Helminth parasites of sturgeon (Acipenser fulvescens) from Lake Nipissing, Ontario, Canada. Proceedings of the $3^{\text {rd }}$ International Congress of Parasitology. 3: 1642-1643.

Anthony, D. D. 1976. The trematode and cestode parasites of walleye (Stizostedion vitreum) from Lake Nipissing, Ontario. Program \& Abstracts, 51 $1^{\text {st }}$ Annual Meeting, American Society of Parasitologists. p. 35.

Anthony, D. D. 1977. A new species of Spinitectus (Nematoda: Rhabdochonidae) from Lake Nipissing, Ontario. Program \& Abstracts, $52^{\text {nd }}$ Annual Meeting, American Society of Parasitologists. p. 70-71.

Anthony, D. D. 1978a. Helminth parasites of lake herring (Coregonus artedii) from Lake Nipissing, Ontario. Program \& Abstracts, $53^{\text {rd }}$ Annual Meeting, American Society of Parasitologists. p. 80-81.

Anthony, D. D. 1978b. Metazoan parasites of percids from Lake Nipissing, Ontario, Canada. Proceedings of the $4^{\text {th }}$ International Congress of Parasitology. Short Communications, Section H. p. 39-40.

Anthony, D. D. 1983. Metazoan parasites of 
esocids from Lake Nipissing, Ontario. Program \& Abstracts, 58 ${ }^{\text {th }}$ Annual Meeting, American Society of Parasitologists. p. 41.

Anthony, D. D. 1987. Helminth parasites of burbot (Lota lota) from Lake Nipissing, Ontario. Program \& Abstracts, $62^{\text {nd }}$ Annual Meeting, American Society of Parasitologists. p. 42-43.

Appy, R. G. \& Cone, D. K. 1982. Attachment of Myzobdella lugubris (Hirudinea: Piscicolidae) to logperch, Percina caprodes and brown bullhead, Ictalurus nebulosus. Transactions of the American Microscopical Society. 101: 135-141.

Arai, H. P. \& Chien, S.-H. 1973. A note on Monogenea (Trematoda) from Albertan fishes. Canadian Journal of Zoology. 51: 1318.

Arai, H. P. and Kussat, R. H. 1967. Observations on the distribution of parasites of certain catostomid fishes of the Bow River, Alberta. Canadian Journal of Zoology. 45: 1287-1290.

Arai, H. P. and Mudry, D. R. 1983. Protozoan and metazoan parasites of fishes from the headwaters of the Parsnip and McGregor rivers, British Columbia: a study of possible parasite transfaunation. Canadian Journal of Fisheries and Aquatic Sciences. 40: 16761684.

Arnason, I. G. 1950. Appendix 18. Feeding habits of some Lake Winnipeg ciscoes, p. 48. In: K. H. Doan. Fisheries Research Board of Canada. Annual Report of the Central Fisheries Research Station for 1950, with Investigators Summaries as Appendices.
Arthur, J. R. and Margolis, L. 1975. Revision of the genus Haplonema Ward and Magath, 1917 (Nematoda: Seuratoidea). Canadian Jounal of Zoology. 53: 736-747.

Arthur, J. R., Margolis, L. and Arai, H. P. 1976. Parasites of fishes of Aishihik and Stevens lakes, Yukon Territory, and potential consequences of their interlake transfer through a proposed water diversion for hydroelectrical purposes. Journal of the Fisheries Research Board of Canada. 33: 2489-2499.

Baggs, E. M. and McT. Cowan, G. I. 1989. The onset of parasitism of young-of-the-year brook char, Salvelinus fontinalis (Mitchill), in Big Northern Pond, Avalon Peninsula, Newfoundland, Canada. Journal of Fish Biology. 35: 317-319.

Bajkov, A. 1930. A study of the whitefish (Coregonus clupeaformis) in Manitoban lakes. Contributions to Canadian Biology and Fisheries, New Series. 5: 441-455.

Bajkov, A. 1930. The genus Leucichthys (ciscoes or tullibees) in Manitoban waters. Contributions to Canadian Biology and Fisheries, New Series. 7: 325-333.

Bajkov, A. D 1933. Report of Fisheries Investigations. Investigations in connection with the broad tapeworm (Diphyllobothrium latum) in Manitoba waters. Manitoba Department of Mines and Natural Resources. Fisheries Branch Report. 19 p.

Baker, M. R. 1984a. Redescription of Dichelyne (Cucullanellus) cotylophora (Ward and Magath, 1917) (Nematoda: Cucullanidae) parasitic in freshwater fishes of eastern North 
America. Canadian Journal of Zoology. 62: 2053-2061.

Baker, M. R. 1984b. On the biology of Dichelyne (Cucullanellus) cotylophora (Ward and Magath, 1917) (Nematoda: Cucullanidae) in perch (Perca flavescens) from Lake Erie, Ontario. Canadian Journal of Zoology. 62: 2062-2073.

Bakke, T. A. and Bailey, R. E. 1987. Phyllodistomum umblae (Fabricius) (Digenea: Gorgoderidae) from British Columbia salmonids: a description based on light and electrom microscopy. Canadian Journal of Zoology. 65: 1703-1712.

Bangham, R. V. 1941. Parasites of fishes of Algonquin Park lakes. Transactions of the American Fisheries Society. 70: 161-171.

Bangham, R. V. 1951. Parasite records by location in 1951: Manitoulin Island and surrounding waters. Unpublished Report. Department of Zoology, University of Toronto, Toronto, Ontario. 27 p.

Bangham, R. V. 1955. Studies on the fish parasites of Lake Huron and Manitoulin Island. American Midland Naturalist. 53: 184194.

Bangham, R. V. and Adams, J. R. 1954. A survey of freshwater fishes from the mainland of British Columbia. Journal of the Fisheries Research Board of Canada. 11: 673-708.

Bangham, R. V. and Hunter, G. W. III. 1939. Studies on fish parasites of Lake Erie. Distribution studies. Zoologica (New York). 24: 385-448.
Bangham, R. V. and Venard, C. E. 1946. Parasites of Algonquin Park Lakes. II. Distribution studies. University of Toronto, Studies in Biology. (Publication of the Ontario Fisheries Research Laboratory.) $15 \mathrm{p}$.

Bashirullah, A. K. M. 1983. Survival and development of Philonema oncorhynchi (Nematoda: Philometridae) in different recipient fish hosts. Rivista di Parassitologia. 44: 489-496.

Becker, C. D. and Katz, M. 1965. Distribution, ecology, and biology of the salmonid leech Piscicola salmositica (Rhynchobdellae: Piscicolidae). Journal of the Fisheries Research Board of Canada. 22: 1175-1195.

Beerman, I. A. N. 1972. The morphology of Octospinifer macilentus (Van Cleave, 1919). M. Sc. Thesis. Department of Biology, University of Calgary, Calgary, Alberta. 196 p.

Beerman, I., Arai, H. P. and Costerton, J. W. 1974. The ultrastructure of the lemnisci and body wall of Octospinifer macilentus (Acanthocephala). Canadian Journal of Zoology. 52: 553-555.

Bell, D. A. and Beverley-Burton, M. 1980. Prevalence and intensity of Capillaria catostomi (Nematoda: Trichuroidea) in white sucker (Catostomus commersoni) on southern Lake Huron, Canada. Environmental Biology of Fishes. 5: 267-271.

Bell, D. A. and Beverley-Burton, M. 1981. The taxonomy of Capillaria spp. (Nematoda: Trichuroidea) in North American freshwater fishes. Systematic Parasitology. 2: 157-169. 
Bell, G. R. and Hoskins, G. E. 1971. Investigations of wild fish mortalities in British Columbia, 1969-1970. Fisheries Research Board of Canada, Technical Report. 245: 19 p.

Bell, G. R. and Margolis, L. 1976. The fish health program and the occurrence of fish diseases in the Pacific region of Canada. Fish Pathology. 10: 115-122.

Bere, R. 1930a. The parasitic copepods of the fish of the Passamoquoddy region. Contributions to Canadian Biology and Fisheries, New Series. 5: 423-430.

Bere, R. 1930b. Parasitic copepods of the Vancouver Island region. Fisheries Research Board of Canada, Manuscript Report (Biology). 259: 3p.

Bérubé, A. and Curtis, M. 1986. Transmission of Diphyllobothrium ditremum to Arctic char (Salvelinus alpinus) in two subarctic Quebec lakes. Canadian Journal of Fisheries and Aquatic Sciences. 43: 1626-1634.

Beverley-Burton, M. 1984. Monogenea and Turbellaria, p. 5-209. In: L. Margolis and Z. Kabata (ed.). Guide to the Parasites of Fishes of Canada. Part 1. Canadian Special Publications in Fisheries and Aquatic Sciences. No. 74.

Beverley-Burton, M., Chisolm, L. A. and Dechtiar, A. O. 1992. Pellucidhaptor eriensis n. sp. and Pellucidhaptor thelostea n. sp. (Monogenea: Dactylogyridae) from catostomids (Cyprinidae) found in Lake Erie, Ontario, Canada. Canadian Journal of Zoology. 69: 2860-2866.

Black, G. A. 1981a. Metazoan parasites as indicators of movements of anadromous brook char (Salvelinus fontinalis) to sea. Canadian Journal of Zoology. 59: 1892-1896.

Black, G. A. 1981b. Population biology of the swim-bladder nematode Cystidicola cristivomeri, in charr, Salvelinus spp. Program \& Abstracts. 20 th Annual Meeting, Canadian Society of Zoologists. Bulletin of the Canadian Society of Zoologists. 12: 33.

Black, G. A. 1982a. Salmincola edwardsii (Copepoda) on brook trout, Salvelinus fontinalis. Program \& Abstracts, $21^{\text {st }}$ Annual Meeting, Canadian Society of Zoologists Bulletin of the Canadian Society of Zoologists. 13(2): 28

Black, G. A. 1982b. Gills as an attachment site for Salmincola edwardsii (Copepoda: Lernaeopodidae). Journal of Parasitology. 68: 1172-1173.

Black, G. A. 1983a. Cystidicola farionis (Nematoda) as an indicator of lake trout (Salvelinus namaycush) of Bering ancestry. Canadian Journal of Fisheries and Aquatic Sciences. 40: 2034-2040.

Black, G. A. 1983b. Origin, distribution, and postglacial dispersal of a swimbladder nematode, Cystidicola stigmatura. Canadian Journal of Fisheries and Aquatic Sciences. 40: 1244-1253.

Black, G. A. 1983c. Taxonomy of a swimbladder nematode, Cystidicola stigmatura (Leidy), and evidence of its decline in the Great Lakes. Canadian Journal of Fisheries and Aquatic Sciences. 40: 643-647.

Black, G. A. 1984a. Swim bladder lesions in 
lake trout (Salvelinus namaycush) associated with mature Cystidicola stigmatura (Nematoda). Journal of Parasitology. 70: 441443.

Black, G. A. 1984b. Morphometrics of Cystidicola stigmatura (Nematoda) in relation to its glacial history. Journal of Parasitology. 70: 967-974.

Black, G. A. and Anderson, R. C. 1982. Zoogeography of Cystidicola cristivomeri in char, Salvelinus spp. Proceedings of the $5^{\text {th }}$ International Congress of Parasitology. Molecular and Biochemical Parasitology. Supplement: 351-352.

Black, G. A. and Fraser, J. M. 1984. Dynamics of prevalence of Ligula intestinalis (L.) In Catostomus commersoni (Lacepede). Jounal of Fish Biology. 25: 139-146.

Black, G. A. and Lankester, M. W. 1980. Migration and development of swim-bladder nematodes, Cystidicola spp. (Habronematoidea), in their definitive hosts. Canadian Journal of Zoology. 58: 1997-2005.

Black, G. A. and Lankester, M. W. 1981a. The biology and parasites of the deepwater sculpin, Myoxocephalus quadricornis thompsoni (Girard), in Burchell Lake, Ontario. Canadian Journal of Zoology. 59: 1454-1457.

Black, G. A. and Lankester, M. W. 1981a. The transmission, life span, and population biology of Cystidicola cristivomeri White, 1941 (Nematoda: Habronematoidea) in char, Salvelinus spp. Canadian Journal of Zoology. 59: 498-509.

Black, G. A. , Montgomery, W. L. and
Whoriskey, F. G. 1983. Abundance and distribution of Salmincola edwardsii (Copepoda) on anadromous brook trout, Salvelinus fontinalis (Mitchill), in the Moisie River system, Quebec. Journal of Fish Biology. 22: 567-575.

Bodaly, R. A., Hrenchuk, C. J. and Fudge, R. J. P. 1983. Post-impoundment experimental fishing, Southern Indian Lake, Manitoba: catches (1982) and Triaenophorous crassus cyst counts in lake whitefish $(1978,1979$, 1982). Canadian Data Report of Fisheries and Aquatic Sciences. No. 401: 75. P.

Boily, F. and Curtis, M. A. 1992. Pattern of Triaenophorus crassus occurrence in the lake whitefish (Coregonus clupeaformis) and cisco (C. artedii) in lakes of the James Bay region, Quebec. Polskie Archiwum Hydrobiologii. 39: 659-665.

Bower, S. M. and Woo, P. T. K. 1977. Morphology and host specificity of Cryptobia catostomi n. sp. (Protozoa: Kinetoplastida) from white sucker (Catostomus commersoni) in southern Ontario. Canadian Journal of Zoology. 55: 1082-1092.

Boyes, J. W. and Anderson, R. C. 1961. Meiotic chromosomes of Cystidicola stigmatura and C. cristivomeri (Nematoda: Spiruroidea). Canadian Journal of Genetics and Cytology. 3: 231-236.

Brassard, P., Curtis, M. A. and Rau, M. E. 1982. Seasonality of Diplostomum spathaceum (Trematoda: Strigeidae) transmission to brook trout (Salvelinus fontinalis) in northern Quebec, Canada. Canadian Journal of Zoology. 60: 2258-2263. 
Brooks, D. R. 1992. New distribution records for Bunodera eucaliae and Bunodera inconstans n. comb. (Digenea: Allocreadiidae: Bunoderinae) with discussion of their phylogenetic relationships. Journal of Parasitology. 78: 779-783.

Buchwald, D, G, and Nursall, J.R. 1969. Triaenophorus crassus in Arctic lampreys of the Northwest Territories, Canada. Journal of the Fisheries Research Board of Canada. 26: 2260-2261.

Budde, W. 1965. Candling for the detection of Triaenophorus crassus cysts in whitefish. Journal of the Fisheries Research Board of Canada. 22: 865-867.

Byrne, P. J. 1992. On the biology of Rhabdochona rotundicaudatum and $R$. cascadilla (Nematoda: Thelaziodidea) in stream fishes from southern Ontario, Canada. Canadian Journal of Zoology. 70: 485-493.

Caira, J. 1989. A revision of the North American papillose Allocreadiidae (Digenea) with independent cladistic analyses of larval and adult forms. Bulletin of the University of Nebraska State Museum. 11: 1-58.

Cameron, T. W. M. 1937. Studies on the heterophyid trematode Apophallus venustus (Ransom, 1920) in Canada. Part II. Life history and bionomics. Canadian Journal of Research, Section D. 15: 38 -51.

Cameron, T. W. M. 1940. Metorchis conjunctus (Cobbold, 1859) Looss, 1899. Report and Proceedings. $3^{\text {rd }}$ International Congress of Microbiology. p. 437-438.

Cameron, T. W. M. 1944. The morphology, taxonomy and life history of Metorchis conjunctus (Cobbold, 1860). Canadian Journal of Research, Section D. 22: 6-16.

Cameron, T. W. M. 1945. Fish-carried parasites in Canada. Canadian Journal of Comparative Medicine. 9:245-254; 283-286; 302-311.

Cameron, T. W. M. 1946. Blackspot and yellow grub in fish. Rod and Gun (Canada). 48(4): 14-15; 48(5): 12-13, 44.

Cameron, T. W. M. and Choquette, L. P. E. 1963. Parasitological problems in northern latitudes, with particular reference to Canada. Polar Record. 11: 567-577.

Cannon, L. G. R. 1970. Studies on the life cycle and ecology of Bunodera sacculata and B. luciopercae (Trematoda: Allocreadiidae) from Algonquin Park. Ph. D. Dissertation. Department of Zoology, University of Toronto, Toronto, Ontario.

Cannon, L. R. G. 1971. The life cycles of Bunodera sacculata and Bunodera luciopercae (Trematoda: Allocreadiidae) in Algonquin Park, Ontario. Canadian Journal of Zoology. 49: 1417-1429.

Cannon, L. R. G. 1972. Studies on the ecology of the papillose allocreadid trematodes of the yellow perch in Algonquin Park, Ontario. Canadian Journal of Zoology. 50: 1231-1239.

Cannon, L. R. G. 1973. Diet and intestinal helminths in a population of perch, Perca flavescens. Journal of Fish Biology. 5: 447457.

Carl, G. C. 1937. Flora and fauna of brackish 
water. Ecology. 18: 446-453.

Chan, G. L. 1980. Study of some parasites of white sucker, Catostomus commersoni (Lacepede), and the fathead minnow, Pimephales promelas Rafinesque, in a reservoir and its contiguous streams. Ph. D. Dissertation. Department of Biology, University of Waterloo, Waterloo, Ontario. 298 p.

Ching, H. L. 1982. Report of the metacestode of Paradilepis simoni Rausch, 1949 (Cyclophyllidea: Dilepididae) from fish in Britisin Columbia. Canadian Journal of Zoology. 60: 184-186.

Ching, H. L. 1984a. Occurrence of the eyefluke, Diplostomum (Diplostomum) baeri bucculentum Dubois et Rausch, 1948 in salmonid fishes of northern British Columbia. Program \& Abstracts. 59 $9^{\text {th }}$ Annual Meeting, American Society of Parasitologists. p. 27.

Ching, H. L. 1984b. Description of Neoechinorhynchus salmonis $\mathrm{sp}$. $\mathrm{n}$. (Acanthocephala: Neoechinorhynchidae) from freshwater fishes of British Columbia. Journal of Parasitology. 70: 286-291.

Ching, H. L. 1985. Occurrence of the eyefluke, Diplostomum (Diplostomum) baeri bucculentum Dubois et Rausch, 1948, in salmonid fishes of northern British Columbia. Canadian Journal of Zoology. 63: 396-399.

Ching, H. L. and Anderson, K. 1983. Description of Eubothrium tulipai (Pseudophyllidea: Amphicotylidae) from northern squawfish in British Columbia. Canadian Journal of Zoology. 61: 981-986.
Ching, H. L. and Parker, L. 1983. Report of water mite larvae in the esophagus and stomach walls of mountain whitefish in British Columbia. Proceedings of the Helminthological Society of Washington. 50: 325-329.

Chinniah, V. C. and Threlfall, W. 1978. Metazoan parasites of fish from the Smallwood Reservoir, Labrador, Canada. Journal of Fish Biology. 13: 203-213.

Choquette, L. P. E. 1947. Phyllodistomum lachancei sp. nov., a trematode from the ureters of Salvelinus fontinalis (Mitchill), with a note on its pathogenicity. Canadian Journal of Research, Section D. 25: 131-134.

Choquette, L. P. E. 1948a. Parasites of freshwater fish. IV. Internal helminths parasitic in speckled trount (Salvelinus fontinalis (Mitchill)) in rivers and lakes in the Laurentide Park, Quebec, Canada. Canadian Journal of Research, Section D. 26: 204-211.

Choquette, L. P. E. 1948b. On the species of the genus Metabronema Yorke and Maplestone, 1926, parasitic in trout and char. Canadian Journal of Research, Section D. 26: 329-333.

Choquette, L. P. E. 1954. A note on the intermediate hosts of the trematode, Crepidostomum cooperi Hopkins, 1931, parasitic in speckled trout (Salvelimus fontinalis (Mitchill)) in some lakes and rivers of the Quebec Laurentide Park. Canadian Journal of Zoology. 32: 374-377.

Choquette, L. P. E. 1955. The life history of the nematode Metabronema salvelini (Fujita, 1920) parasitic in the speckled trout, 
Salvelinus fontinalis (Mitchill), in Quebec. Canadian Journal of Zoology. 33: 1-4.

Choudhury, A. and Dick, T. A. 1991. Parasites of the lake sturgeon, Acipenser fulvescens (Chondrostei: Acipenseridae) from the prairie region of Canada. Program \& Abstracts. $30^{\text {th }}$ Annual Meeting, Canadian Society of Zoologists. Bulletin of the Canadian Society of Zoologists. 22: 30 .

Choudhury, A. and Dick, T. A. 1992. Spinitectus acipenseri $\mathrm{n}$. sp. (Nematoda: Cystidicolidae) from the lake sturgeon Acipenser fulvescens (Rafinesque) in Canada. Systematic Parasitology. 22: 131-140.

Choudhury, A. and Dick, T. A. 1993. Parasites of lake sturgeon, Acipenser fulvescens (Chondrostei: Acipenseridae), from central Canada. Journal of Fish Biology. 42: 571-584.

Choudhury, A. and Dick, T. A. 1994. Natural anti-phosphorycholine (PC) antibodies in lake sturgeon, Acipenser fulvescens Rafinesque, 1817 (Chondrostei: Acipenseridae). Fish and Shellfish Immunology. 4: 399-401.

Choudhury, A. and Dick, T. A. 1996a. Observations on the morphology of Truttaedacnitis clitellarius and T. lebedevi (Nematoda: Cucullanidae) from acipenserids and synonymy of the two species. Systematic Parasitology. 33: 89-99.

Choudhury, A. and Dick, T. A. $1996 \mathrm{~b}$. Diclybothrium atriatum n. sp. (Monogenea: Diclybothriidae) from North American acipenserid fishes with observations on Diclybothrium armatum and Diclybothrium hamulatum. Journal of Parasitology. 82: 965976.
Choudhury, A. and Dick, T. A. 1996c. Observations on the morphology, systematics, and biogeography of the genus Truttaedacnitis (Nematoda: Cucullanidae). Journal of Parasitology. 82: 977-987.

Choudhury, A., Dick, T. A., Holloway, H. and Ottinger, C. 1990. The lake sturgeon Acipenser fulvescens (Chondrostei, Acipenseridae) in Canada: preliminary studies on parasitofauna and immunological parameters. p. 310-321. In: Interbasin Biota Transfer, North Dakota Water Quality Symposium, North Dakota State University, Fargo, North Dakota.

Clemens, W. A., Boughton, R. V. and Rattenbury, J. A. 1945. A preliminary report on a fishery survey of Teslin Lake, British Columbia. British Columbia Department of Fisheries. Report for 1944. p. 70-75.

Clemens, W. A., Rawson, D. S. and McHugh, J. L. 1939. A biological survey of Okanogan Lake, British Columbia. Fisheries Research Board of Canada. Bulletin 56: $70 \mathrm{p}$.

Clifford, H. F. 1991. 26. Branchiura, p. 167. In: Aquatic Invertebrates of Alberta. University of Alberta Press, Edmonton, Alberta.

Collins, J. J. and Dechtiar, A. O. 1974. Parasite fauna of kokanee salmon (Oncorhynchus nerka) introduced into Lake Huron. Journal of the Fisheries Research Board of Canada. 31: 1818-1821.

Cone, D. K. 1978. Systematic position of Urocleidus adspectus Mueller, 1936 (Monogenea: Ancyrocephalinae) of Perca flavescens. Canadian Journal of Zoology. 56: 
608-612.

Cone, D. K. 1979a. Hatching of Urocleidus adspectus Mueller, 1936 (Monogenea: cephalinae). Canadian Journal of Zoology. 57 : 833-837.

Cone, D. K. 1979b. The oncomiracidium of Urocleidus adspectus Mueller, 1936 (Monogenea: Ancryocephalinae). Canadian Journal of Zoology. 57: 1098-1103.

Cone, D. K. 1979c. Development of the haptor of Urocleidus adspectus Mueller, 1936 (Monogenea: Ancyrocephalinae). Canadian Journal of Zoology. 57: 1896-1904.

Cone, D. K. 1980. The Monogenea of some freshwater fishes of New Brunswick, Canada. Journal of Parasitology. 66: 178-180.

Cone, D. K., Beverley-Burton, M., Wiles, M. and McDonald, T. E. 1983. The taxonomy of Gyrodactylus (Monogenea) parasitizing certain salmonid fishes of North America, with a description of Gyrodactylus nerkae n. sp. Canadian Journal of Zoology. 61: 2587-2597.

Cone, D. K. and Burt, M. D. B. 1981. The invasion route of the gill parasite Urocleidus adspectus Mueller, 1936 (Monogenea: Ancyrocephalinae). Canadian Journal of Zoology. 59: 2166-2171.

Cone, D. K. and Burt, M. D. B. 1982a. The host specificity of Urocleidus adspectus Mueller, 1936 (Monogenea: Ancyrocephalinae). Journal of Parasitology. 68: 1168-1170.

Cone, D. K. and Burt, M. D. B. 1982b. The behaviour of Urocleidus adspectus Mueller,
1936 (Monogenea) on the gills of Perca flavescens. Canadian Journal of Zoology. 60: 3237-3240.

Cone, D. K. and Burt, M. D. B. 1985. Population biology of Urocleidus adspectus Mueller, 1936 (Monogenea) on Perca flavescens in New Brunswick. Canadian Journal of Zoology. 63: 272-277.

Cone, D. K. and Cussack, R. 1988. A study of Gyrodactylus colemanensis Mizelle and Kritsky, 1967 and Gyrodactylus salmonis (Yin and Sproston, 1948)(Monogenea) parasitizing captive salmonids in Nova Scotia. Canadian Journal of Zoology. 66: 409-415.

Cone, D. K. and Cussack, R. 1989. Infrapopulation dispersal of Gyrodactylus colemanensis Mizelle and Kritsky, 1967 (Monogenea) on fry of Salmo gairdneri. Journal of Parasitology. 75: 702-706.

Cone, D. K. and Dechtiar, A. O. 1984. Gyrodactylus fryi $\mathrm{n}$. sp. (Monogenea) from Esox masquinongy Mitchill in Ontario. Canadian Journal of Zoology. 62: 1089-1090.

Cone, D. K. and Dechtiar, A. O. 1986. On Gyrodactylus katherineri Malmberg, 1964, G. lotae Gussev, 1953 and G. lucii Kulakovskaya, 1952 from host fishes in North America. Canadian Journal of Zoology. 64: 637-639.

Cone, D. K. and Odense, P. H. 1984. Pathology of five species of Gyrodactylus Nordmann, 1832 (Monogenea). Canadian Journal of Zoology. 62: 1084-1088.

Cone, D. K. and Ryan, P. M. 1984. Population sizes of metazoan parasites of brook trout 
(Salvelinus fontinalis) and Atlantic salmon (Salmo salar) in a small Newfoundland lake. Canadian Journal of Zoology. 62: 130-133.

Cone, D. K. and Wiles, M.. 1985. The systematics and zoogeography of Gyrodactylus species (Monogenea) parasitizing gasterosteid fishes in North America. Canadian Journal of Zoology. 63: 956-960.

Cone, D. K. and Wiles, M. 1989. Ultrastructural study of attachment of Gyrodactylus colemanensis (Monogenea) to fins of fry of Salmo gairdneri. Proceedings of the Helminthological Society of Washington. 56: 29-32.

Conley, D. C. and Curtis, M. A. 1988. Salmincola edwardsii (Crustacea: Copepoda): a parasitic pathogen of pond-cultured brook trout in Quebec. Bulletin of the Aquaculture Association of Canada. 88-4. 107-109.

Conley, D. C. and Curtis, M. A. 1992. A temperature and photoperiod experiment on Salmincola edwardsii (Crustacea: Copepoda): a possible model for sea lice research. Bulletin of the Aquaculture Association of Canada. 923: 25-27.

Conley, D. C. and Curtis, M. A. 1993. Effects of temperature and photoperiod on the duration of hatching, swimming and copepodid survival of the parasitic copepod Salmincola edwardsii. Canadian Journal of Zoology. 71: 922-926.

Conley, D. C. and Curtis, M. A. 1994. Larval development of the parasitic copepod Salmincola edwardsii on brook trout (Salvelinus fontinalis). Canadian Journal of
Zoology. 72: 154-159.

Cooper, A. R. 1915. Trematodes from marine and freshwater fishes, including one species of ectoparasitic turbellarian. Transactions of the Royal Society of Canada, Series 3. 9(4): 181205.

Cooper, A. R. 1919. North American pseudophyllidean cestodes from fishes. Illinois Biological Monographs. 4: 289-541.

Cooper, A. R. 1921. Trematodes and cestodes of the Canadian ArcticExpedition, 1913-1918. Report of the Canadian Arctic Expedition, 1913-1918. 9(G-H); 1-27.

Couillard, C. M., Hodson, P. V., Gagnon, P. and Dodson, J. J. 1995. Lesions and parasites in white suckers, Catostomus commersoni, in bleached-kraft pulp mill- contaminated and reference rivers. Environmental Toxicology and Chemistry. 14: 1051-1060.

Courtney, C. C. 1987. Ecology of helminths of catostomids from western Lake Superior. Program \& Abstracts. $62^{\text {nd }}$ Annual Meeting, American Soceity of Parasitologists. p. 29.

Crampton, E. W. 1960. Effect of ingestion of Triaenophorus pleurocercoids on the nutritional ch:-acteristics of whitefish fillets in the rations of puppies. Journal of the Fisheries Research Board of Canada. 17: 81-90.

Crossman, E. J. 1962. Black spot parasite in fishes. Information Leaflet. Department of Ichthyology and Herpetology, Royal Ontarion Museum. April 1962: 4 p.

Cuerrier, J. -P. And Schultz, F. H. 1957. Studies on flake trout and common whitefish 
in Waterton Lakes, Waterton Lakes National Park, Alberta. Canadian Wildlife Service, Wildlife Management Bulletin, Series 3. No. 5: $41 \mathrm{p}$.

Curtis, M. A. 1981. Observations on the occurrence of Diplostomum spathaceum and Schistocephalus sp. in ninespine sticklebacks (Pungitius pungitius) from the Belcher Islands, Northwest Territories, Canada. Journal of Wildlife Diseases. 17: 241-246.

Curtis, M. A. 1982. Host-parasite interactions in Arctic and sub-Arctic lakes, p. 41-57. In: E. Meerovitch (ed.). Aspects of Parasitology - a Festschrift dedicated to the $50^{\text {th }}$ Anniversary of the Institute of Parasitology of McGill University, 1932-1982. McGill University, Montreal, Canada. 485 p.

Curtis, M. A. 1984. Diphyllobothrium spp. and the Arctic charr: parasite acquisition and its effects on a lake-resident population, p. 395-411. In: L. Johnson \& B. L. Burns (ed.). Biology of the Arctic Charr: Proceedings of the International Symposium on Arctic Charr. Winnipeg, Manitoba. University of Manitoba Press, Winnipeg.

Curtis, M. A. 1988. Experimental depletion of a brook trout population to improve yield and decrease parasite abundance. Bulletin of the Aquaculture Association of Canada. 88-4: 22 24.

Curtis, M. A. and Rau, M. E. 1980. The geographical distribution of diplostomiasis (Trematoda: Strigeidae) in fishes from northern Quebec, Canada, in relation to the calcium ion concentration of lakes. Canadian Journal of Zoology. 58: 1390-1394.
Cusack, R. 1986. Development of Gyrodactylus colemanensis Mizelle and Kritsky, 1967 (Monogenea) and effect on fry of Salmo gairdneri Richardson. Journal of Parasitology. 72: 663-668.

Cusack, R. and Cone, D. K. 1986. Gyrodactylus salmonis (Yin and Sproston, 1948) parasitizing fry of Salvelinus fontinalis (Mitchill). Journal of Wildlife Diseases. 22: 209-213.

Cusack, R., Rand, T. and Cone, D. K. 1988. Studies on the bacterial microcolonies on the body surface of Gyrodactylus colemanensis parasitizing Salmo gairdneri. Journal of Fish Diseases. 11: 271-274.

Cushing, H. B. and Bacal, N. L. 1932. Diphyllobothrium latum (fish tapeworm) infestation in eastern Canada. Canadian Medical Journal. 30: 377-384.

Dais, J. M. 1990. Biochemical and ultrastructural characterization of Diphyllobothrium spp. (Cestoidea). Ph. D. Dissertation, Department of Biological Sciences, University of Calgary, Calgary, Alberta. 149 p.

Daniels, B. and Freeman, R. S. 1976. A review of the genus Actinobdella Moore, 1901 (Annelida, Hirudinea). Canadian Journal of Zoology. 54: 2112-2117.

Dechtiar, A. O. 1966. A new species of Phyllodistomum (Trematoda: Gorgoderidae) from Coregonus clupeaformis (Mitchill) from Lake of the Woods (Ontario). Canadian Journal of Zoology. 44: 135-140.

Dechtiar, A. O. 1967. Neodiscocotyle 
carpioditis n. gen., n. sp., monogenetic trematode(Discocotylidae: Neodiscocotylinae subfam. n.) from the gills of the quillback, Carpiodes cyprinus (Le Sueur) of Lake Erie. Canadian Journal of Zoology. 45: 473-478.

Dechtiar, A. O. 1968. Neoechinorhynchus carpiodi n. sp. (Acanthocephala: Neoechinorhynchidae) from quillback of Lake Erie. Canadian Journal of Zoology. 46: 201204.

Dechtiar, A. O. 1969. Two new species of monogenetic trematodes (Trematoda: Monogenea) from nasal cavities of catostomid fishes. Journal of the Fisheries Research Board of Canada. 26: 865-869.

Dechtiar, A. O. 1971. Neoechinorhynchus pungitius n. sp. (Acanthocephala: Neoechinorhynchidae) from ninespine stickleback of Lake Huron. Canadian Journal of Zoology. 49: 483-486.

Dechtiar, A. O. 1972a. Parasites of fish from Lake of the Woods, Ontario. Journal of the Fisheries Research Board of Canada. 29: 275 283.

Dechtiar, A. O. 1972b. New parasite records for Lake Erie fish. Great Lakes Fisheries Commission, Technical Report. 17: 20 p.

Dechtiar, A. O. 1972c. Systematic status of Tetraonchus loftusi n. sp. (Monogenoidea: Tetraonchidae) and comparative studies of $T$. monenteron (Wagner, 1857) Diesing, 1858, and T. variabilis Mizelle and Webb, 1953. Canadian Journal of Zoology. 50: 1489-1495.

Dechtiar, A. O. 1974a. Cleidodiscus baldwini n. sp. (Monogenoidea: Ancyrocephalinae) from the gills of the trout-perch (Percopsis omiscomaycus) of Lake Huron. Journal of the Fisheries Research Board of Canada. 31: 163165.

Dechtiar, A. O. 1974b. Dactylogyrus buddi n. sp. (Monogenoidea: Dactylogyridae) from the gills of the sculpins of Lake Huron. Canadian Journal of Zoology. 52: 861-863.

Dechtiar, A. O. and Berst, A. H. 1978. Parasites of splake (Salvelinus fontinalis X S. namaycush). Proceedings of the Helminthological Society of Washington. 45: 249-254.

Dechtiar, A. O. and Christie, W. J. 1988. Survey of the parasite fauna of Lake Ontario fishes, 1961 to 1971 , p. 66-95. In: S. J. Nepszy (ed.). Parasites of Fishes of the Canadian Waters of the Great Lakes. Great Lakes Fishery Commission, Technical Report. No. 51.

Dechtiar, A. O., Collins, J. J. and Reckahn, J. A. 1988. Survey of the parasite fauna of Lake Huron fishes, 1961 to 1971 , p. 19-48. In: S. J. Nepszy (ed.). Parasites of Fishes in the Canadian Waters of the Great Lakes. Great Lakes Fishery Commission, Technical Report. No. 51.

Dechtiar, A. O. and Dillon, W. A. 1974. Redescription of Anonchohaptor anomalum Mueller, 1938 and a description of Icelanonchohaptor fyviei n. sp. (Monogenoidea: Dactylogyridae). Journal of the Fisheries Research Board of Canada. 31: 1863-1866.

Dechtiar, A. O. and Lawrie, A. H. 1938. Survey of the parasite fauna of Lake Superior 
fishes, 1961 to 1971 , p. 1-18. In: S. J. Nepszy (ed.). Parasites of Fishes in the Canadian Waters of the Great Lakes. Great Lakes. Great Lakes Fishery Commission, Technical Report. No. 51.

Dechtiar, A. O. and Loftus, K. H. 1965. Two new hosts for Cyathocephalus truncatus (Pallas, 1781) (Cestoda: Cyathocephalidae) in Lake Huron. Canadian Journal of Zoology. 43 : 407-408.

Dechtiar, A. O. , MacLean, J. A. and Nepszy, S. J. 1989. Parasites of fishes from Algonquin Park lakes. Ontario Fisheries Technical Report Series. No. 29: 19 p.

Dechtiar, A. O. and Nepszy, S. J. 1988. Survey of the parasite fauna of selected fish species from Lake Erie, p. 49 -65. In: S. J. Nepszy (ed.). Parasites of Fishes in the Canadian Waters of the Great Lakes. Great Lakes Fishery Commission, Technical Report. No. 51.

Després;, L. Adamson, M. L. and McDonald, T. E. 1995. Development of a diagnostic molecular marker for Philonema spp. (Nematoda: Dracunculoidea) infecting salmonids in British Columbia. Canadian Journal of Fisheries and Aquatic Sciences. 52(Supplement): 129-133.

Detwiler, J. D. 1941. Parasitic infestations of fish. Canadian Public Health Journal. 32: 293-300.

De Vos, T. and Dick, T. A. 1989. Differentiation between Diphyllobothrium dendriticum and D. latum using isozymes, restriction profiles and ribosomal gene probes. Systematic Parasitology. 13: 161-166.
De Vos, T., Szalai, A. J. and Dick, T. A. 1990. Genetic and morphological variability in a population of Diphyllobothrium dendriticum (Nitzsch, 1824). Systematic Parasitology. 16: 99-105.

Dextrase, A. J. and Lankester, M. W. 1987. The biology of Cystidicola farionis (Habronematoidea) in salmonid fishes. Program \& Abstracts. 26 ${ }^{\text {th }}$ Annual Meeting, Canadian Society of Zoologists. Bulletin of the Canadian Society of Zoologists. 18(2): 22.

Dick, T. A. 1987. The atrium of the fish heart as a site for Contracaecum spp. larvae. Journal of Wildlife Diseases. 23: 328-330.

Dick, T. A. and Choudhury, A. 1995. Cestoidea (Phylum Platyhelminthes), p. 391414. In: P. T. K. Woo (ed.). Fish Diseases and Disorders. CAB International, Wallingford, UK.

Dick, T. A., Holloway, H. L. and Choudhury, A. 1991. Polypodium sp. (Coelenterata) from the lake sturgeon (Acipenser fulvescens Rafinesque) in the prairie region of Canada. Journal of Parasitology. 77: 483-484.

Dick, T. A., Papst, M. H. and Paul, H. C. 1987. Rainbow trout (Salmo gairdneri) stocking and Contracaecum spp. Journal of Wildlife Diseases. 23: 242-247.

Dick, T. A. and Poole, B. C. 1985. Identification of Diphyllobothrium dendriticum and Diphyllobothrium latum from some freshwater fishes of central Canada. Canadian Journal of Zoology. 63: 196-201.

Dick, T. A. and Rosen, R. 1981. Identification of Diplostomum spp. from the eyes of lake 
whitefish, Coregonus clupeaformis (Mitchill), based on experimental infection of herring gull chicks, Larus argentatus Pontoppidan. Canadian Journal of Zoology. 59: 1176-1179.

Dick, T. A. and Rosen, R. 1982. Experimental infections of whitefish, Coregonus clupeaformis (Mitchill), with Triaenophorus crassus Forel. Journal of Fish Diseases. 5: 8386.

Dick, T. A. and Watson, R. 1977. The whitefish-pike parasite Triaenophorus crassus. Manitoba Nature. Winter 1977: 26-31.

Dickinson, A. B. and Threlfall, W. 1977. Some parasites of Fundulus diaphanus and Pungitius pungitius from insular Newfoundland. Proceedings of the Helminthological Society of Washingtom. 43: 86-87.

Dickson, T. W. Parasites of some fishes of Manitoba. M. Sc. Thesis. Department of Zoology, University of Manitoba, Winnipeg, Manitoba.

Dies, K. 1990. Leech infestation in trout. Canadian Veterinary Journal. 31: 119.

Doan, K. H. 1944. Appendix No. 2. Whitefish infestation in Prairie provinces, p. 9-10. In: K. H. Doan. Fisheries Research Board of Canada. Annual Report of the Central Fisheries Research Station for 1944, with Investigators' Summaries as Appendices.

Doan, K. H. 1945a. Appendix No. 1. Experimental control of jackfish, p. 6-8. In: K.H. Doan. Fisheries Research Board of Canada. Annual Report of the Central Fisheries Research Station for 1944, with
Investigators Summaries as Appendices.

Doan, K. H. 1945b. The control of jackfish, Exox lucius, in Heming Lake, Manitoba, in relation to the parasitization of whitefish by the tapeworm Triaenophorus crassus. Fisheries Research Board of Canada. Manuscript Report (Biology) No. 462: 35 p.

Doan, K. H. 1945c. Appendix No. 2. Whitefish infestation survey, p. 8-10. In: K. H. Doan. Fisheries Research Board of Canada. Annual Report of the Central Fisheries Research Station for 1944, with Investigators' Summaries as Appendices.

Doan, K. H. 1945d. Appendix No. 4. Parasites in Lake Winnipegosis tullibee, p. 11-12. In: K. H. Doan. Fisheries Research Board of Canada. Annual Report of the Central Fisheries Research Station for 1944, with Investigators' Summaries as Appendices.

Doan, K. H. 1945e. Appendix V. Report for 1944 of the Central Fisheries Research Station, Winnepeg, Man., p. 37-38. In: A. T. Cameron. Annual Report of the Fisheries Research Board of Canada for 1944.

Doan, K. H. 1946a. Appendix No. 1 Jackfish control work and whitefish infestation, p. 9. In: K. H. Doan. Fisheries Research Board of Canada. Annual Report of the Central Fisheries Research Station for 1946, with Investigators' Summaries as Appendices.

Doan, K. H. 1946b. Appendix V. Report for 1945 of the Central Fisheries Research Station, Winnipeg, Man., p. 41. In: A. T. Cameron. Annual Report of the Fisheries Research Board of Canada for the year 1945. 
Doan, K. H. 1947a. Appendix No. 1. Jackfish control work and whitefish infestation, p. 910. In: K. H. Doan. Fisheries Research Board of Canada. Annual Report of the Central Fisheries Research Station for 1947, with Investigators' Summaries as Appendices.

Doan, K. H. 1947b. Appendix V. Report for the Central Fisheries Station, Winnepeg, Man., p. 40-41. In: J. R. Dymond. Annual Report of the Fisheries Research Board of Canada for the year 1946.

Doan, K. H. 1948a. Appendix No. 1. Jackfish control and whitefish quality, p. 11-12. In: K. H. Doan. Fisheries Research Board of Canada. Annual Report of the Central Fisheries Research Station for 1948, with Investigators' Summaries as Appendices.

Doan, K. H. 1948b. Appendix VI. Report for 1947 of the Central Fisheries Research Station, Winnepeg, Man., p. 50-51. In: G. B. Reed. Annual report of the Fisheries Research Board of Canada for the year 1947.

Doan, K. H. 1949a. Appendix No. 6. The killing of pike to reduce whitefish infestation in Heming Lake, Manitoba, p. 26-28. In: K. H. Doan. Fisheries Research Board of Canada. Annual Report of the Central Fisheries Research Station fo 1949, with Investigators' Summaries as Appendices.

Doan, K. H. 1949b. Control of the pikewhitefish tapeworm in central Canada (Abstract). In: W. A. Clemens. Fisheries Research Board of Canada. Report of Delegates and Report of the Fisheries of New Zealand, $7^{\text {th }}$ Pacific Science Congress.

Doan, K. H. 1950. Appendix VIII. Report for the Central Fisheries Research Station, Winnepeg, Man., p. 66-69. In: G. B. Reed and J. R. Dymond. Annual Report of the Fisheries Research Board of Canada for the year 1949.

Doan, K. H. 1951. Appendix No. 1. Whitefish quality, p. 1-3. In: K. H. Doan. Fisheries Research Board of Canada. Annual Report of the Central Fisheries Research Station for 1951, with Investigators' Summaries as Appendices.

Doan, K. H. 1953. Control of the pikewhitefish tapeworm in central Canada. Proceedings of the $7^{\text {th }}$ Pacific Science Congress. 4(Zoology): 539-547.

Donald, D. B. and Kooyman, A. H. 1974 Status of the goldeye (Hiodon alosoides) populations in the Peace-Athabasca delta of Wood Buffalo National Park (1971-1973). Canadian Wildlife Service, Manuscript Report. $63 \mathrm{p}$.

Dowsett, J. A. and Lubinsky, G. A. 1966. Maturation of Clinostomum marginatum (Trematoda) in laboratory mice. Canadian Journal of Zoology. 44: 496.

Drouin, T.E. 1982. Parasites and parasitism of the whitefish of Lake McGregor and Travers Reservoir, Alberta. Ph. D. Dissertation. Department of Biology, University of Calgary, Calgary, Alberta. 280 p.

Dubois, N., Marcogliese, D. J. and Magnan, P. 1996. Effects of the introduction of white sucker, Catostomum commersoni, on the parasite fauna of brook trout, Salvelinus fontinalis. Canadian Journal of Zoology. 74: 1304-1312. 
Dunbar, M. J. and Hildebrand, H. H. 1952. Contribution to the study of the fishes of Ungava Bay. Journal of the Fisheries Research Board of Canada. 9: 83-128.

Dunn, I. J., Russell, L. R. and Adams, J. R. 1983. Cecal histopathology caused by Truttaedacnitis truttae (Nematoda: Cucullanidae) in rainbow trout, Salmo gairdneri. International Journal for Parasitology. 13: 441-445.

Eaton, R. D. P. 1975. Metorchiasis - a Canadian zoonosis. Epidemiological Bulletin. 19: 62-68.

Ekbaum, E. 1935. Notes on the species of Triaenophorus in Canada. Journal of Parasitology. 21: 260-263.

Ekbaum, E. 1936. Notes on the genus Cystidicola in Canadian fishes. Canadian FieldNaturalist. 50: 8-11.

Ekbaum, E. 1937. On the maturation and the hatching of the eggs of the cestode Triaenophorus crassus Forel from Canadian fish. Journal of Parasitology. 23: 293-295.

Evans, W. S. 1963a. Amphimerus pseudofelineus (Ward, 1901) (Digenea: Opisthorchidae) and its second intermediate host in Manitoba. Canadian Journal of Zoology. 41: 649-651.

Evans, W. S. 1963b. Fish-carried liver parasites of Manitoba mammals. M. Sc. Thesis. Department of Zoology. University of Manitoba, Winnipeg, Manitoba. 104 p.

Fallis, A. M. 1934. Preliminary report on the parasites collected at the Ontario Fisheries
Research Laboratory. Ontario Fisheries Research Laboratory Report. 3 p.

Fantham, H. B. and Porter, A. 1948. The parasitic fauna of vertebrates in certain Canadian fresh waters, with some remarks on their ecology, structure and importance. Proceedings of the Zoological Soceity of London, Series B. 117: 609-649.

Fillion, D. B. 1961. Parasites of the burbot (Lota lota) in Alberta. Unpublished Report. Department of Zoology, University of Alberta, Edmonton, Alberta. 12 p.

Fischer, H. 1972. Studies on the life cycles and ecology of Proteocephalus ambloplitis, $P$. fluviatilis and $P$. pearsei (Cestoda) from Lake Opeongo, Algonquin Park. Ph. D. Dissertation. Department of Zoology, University of Toronto, Toronto, Ontario. 380 p.

Fischer, H. 1974. Studies on the life cycles and ecology of Proteocephalus ambloplitis, $P$. fluviatilis and $P$. pearsei (Cestoda) from Lake Opeongo, Algonquin Park. Dissertation Abstracts International. 34B: 3644.

Fischer, H. and Freeman, R. S. 1973. The role of plerocercoids in the biology of Proteocephalus ambloplitis (Cestoda) maturing in smallmouth bass. Canadian Journal of Zoology. 51: 133-141.

Frantsi, C., Flewelling, T. C. and Tidswell, K. G. 1975. Investigations on corynebacterial kidney disease and Diplostomulum sp. (eyefluke) at Margaree Hatchery, 1972-1973. Canada Department of Fisheries, Fisheries and Marine Service, Research Development Technical Report. MAR/T-75-9: 30 p. 
Freeman, R. S. 1961. The life history of Proteocephalus parallacticus MacLulich, 1843, from lake trout. Journal of Parasitology. 47(4, Sect. 2): 57.

Freeman, R. S. 1964a. On the biology of Proteocephalus parallacticus MacLulich (Cestoda) in Algonquin Park, Canada. Canadian Journal of Zoology. 42: 387-408.

Freeman, R. S. 1964b. Flatworm problems in fish. Canadian Fish Culturist. No. 32: 11-18.

Freeman, R. S. and Thompson, B. H. 1969. Observations on the transmission of Diphyllobothrium sp. (Cestode) to lake trout in Algonquin Park, Canada. Journal of the Fisheries Research Board of Canada. 26: 871878.

Freese, M. 1969. Ultrasonic inspection of parasitized whole fish. In: R. Kreuzer (ed.). Fish Inspection and Quality Control. Paper FTC/69/0/12. FAO Technical Conference, Halifax, Nova Scotia. 13 p.

Freese, M. 1970. Distribution of Triaenophorus crassus parasites in whitefish flesh and its significance to automatic detection of the parasites by ultrasound. Journal of the Fisheries Research Board of Canada. 27: 271-279.

Freese, M. 1971. Ultrasonic inspection of parasitized whole fish, p. 256-262. In: R. Kreuzer (ed.). Fish Inspection and Quality Control. Fishing News (Books) Ltd., London, UK.

Freese, M. 1973. The automatic detection of parasites by the ultrasonic echo method. Fisheries Research Board of Canada.
Technical Report. No. 346: 81 p.

Frimeth, J. P. 1987a. A survey of the parasites of nonanadromous and anadromous brook charr (Salvelinus fontinalis) in the Tabusintac River, New Brunswick, Canada. Canadian Journal of Zoology. 65: 1354-1362.

Frimeth, J. P. 1987b. Potential use of certain parasites of brook charr (Salvelinus fontinalis) as biological indicators in the Tabusintac River, New Brunswick, Canada. Canadian Journal of Zoology. 65: 989-995.

Frost, N. 1940. A preliminary study of Newfoundland trout. Newfoundland Department of Natural Resources. Research Bulletin. 9(Fisheries): $30 \mathrm{p}$.

Fuller, W. A. 1955. The inconnu (Stenodus leucichthys mackenziei) in Great Slave Lake and adjoining waters. Journal of the Fisheries Research Board of Canada. 12: 768-780.

Gaboury, M. N. 1978. Biological investigations on brook trout (Salvelinus fontinalis) populations in the Long SpruceLimestone area from 1975 to 1977 and implications of hydroelectric development of the lower Nelson River. Manitoba Department of Renewable Resources \& Transportation Services, Fisheries Research Section, Research Manuscript Report. No. 78-49: 146 p.

Glenn, C. L. 1980. Seasonal parasitic infections in mooneye, Hiodon tergisus (LeSueur), from the Assiniboine River. Canadian Journal of Zoology. 58: 252-257.

Godfrey, H. 1968. Some observations on juvenile chum, chinook, and coho salmon taken in the waters adjacent to Georgia Strait 
in 1965. Fisheries Research Board of Canada. Manuscript Report. No. 955: 19p.

Godin, J. -G. and Sproul, C. D. 1988. Risk taking in parasitized sticklebacks under threat of predation: effects of energetic need and food availability. Canadian Journal of Zoology. 66: 2360-2367.

Gordon, D., Croll, N. A. and Rau, M. E. 1978. Les parasites des animaux sauvages du Québec. I. Les parasites des poissons et des mamifêres de la region de Schefferville. Naturaliste Canadienne (Québec). 105: 55.

Gordon, D. M. and Rau, M. E. 1982a. Temporal changes in the frequency distribution of Apatemon gracilis in brook sticklebacks (Culaea inconstans). Parasitology. 84(1): iii.

Gordon, D. M. and Rau, M. E. 1982 b. Possible evidence for mortality induced by the parasite Apatemon gracilis in a population of brook sticklebacks (Culaea inconstans). Parasitology. 84: 41-47.

Greenwood, S. J. and Baker, M. R. 1987. Cystidicoloides ephemeridarum (Linstow, 1872) (Nematoda) in speckled trout, Salvelinus fontinalis, from southern Ontario. Canadian Journal of Zoology. 65: 2589-2593.

Grey, A. J. and Mackiewicz, J. S. 1980. Chromosomes of caryophyllidean cestodes: diploidy, triploidy, and parthenogenesis in Glaridacris catostomi. International Journal for Parasitology. 10: 397-407.

Hanek, G. 1971. Study on monogenetic trematodes of the fishes from the Bay of Quinte area, Ontario. M. Sc. Thesis. Department of Biology, University of
Waterloo, Waterloo, Ontario. 86 p.

Hanek, G. and Fernando, C. H. 1971. Monogenetic trematodes from the Bay of Quinte area, Ontario. II. Genus Gyrodactylus Nordmann, 1832. Canadian Journal of Zoology. 49: 1331-1341.

Hanek, G. and Fernando, C. H. 1972a. Monogenetic trematodes from the Bay of Quite area, Ontario. III. Genera Actinocleidus, Cleidodiscus, Urocleidus, and Tetraonchus. Canadian Journal of Zoology. 50: 1303-1312.

Hanek, G. and Fernando, C. H. 1972 b. Monogenetic trematodes from the Bay of Quinte area, Ontario. IV. Genus Dactylogyrus Diesing, 1850, with provisional host-parasite and parasite-host lists. Canadian Journal of Zoology. 50: 1313-1317.

Hanek, G. and Molnar, K. 1974. Parasites of freshwater and anadromous fishes from Matamek River system, Quebec. Journal of the Fisheries Research Board of Canada. 31: 1135-1139.

Hanek, G., Molnar, K. and Fernando, C. H. 1975. New and previously known Dactylogyrus spp. from southern Ontario. Journal of Parasitology. 61: 421-426.

Hanek, G. and Threlfall, W. 1969a. Therestina gasterostei (Pagenstecher, 1861) (Copepoda: Ergasilidae) from Gasterosteus wheatlandi Putnan, 1867. Canadian Journal of Zoology. 47: 627-629.

Hanek, G. and Threlfall, W. $1969 \mathrm{~b}$. Monogenetic trematodes from Newfoundland, Canada. I. New species of the genus Gyrodactylus Nordmann, 1832. Canadian 
Journal of Zoology. 47: 951-955.

Hanek, G. and Threlfall, W. 1969c. Digenetic trematodes from Newfoundland, Canada. 2. Two new species from Gasterosteus aculeatus Linnaeus, 1758. Canadian Journal of Zoology. 47: 1086-1087.

Hanek, G. and Threlfall, W. 1970a. Parasites of the threespine stickleback (Gasterosteus aculeatus) in Newfoundland and Labrador. Journal of the Fisheries Research Board of Canada. 27: 901-907.

Hanek, G. and Threlfall. W. 1970b. Parasites of the ninespine stickleback Pungitius pungitius (L.) In Newfoundland and Labrador. Canadian Journal of Zoology. 48: 600-602.

Hanek, G. and Threlfall, W. 1970c. Ergasilus auritus Markewitsch, 1949 (Copepoda: Ergasilidae) from Gasterosteus aculeatus Linnaeus, 1758 in Newfoundland. Canadian Journal of Zoology. 48: 185-187.

Hare, G. M. and Frantsi, C. 1974. Abundance and potential pathology of parasites infecting salmonids in Canadian Maritime hatcheries. Journal of the Fisheries Research Board of Canada. 31: 1031-1036.

Harkness. W. J. K. and Dymond, J. R. 1961. The lake sturgeon. The history of its fishery and problems of conservation. Ontario Department of Lands \& Forests. Fish and Wildlife Branch Report. 121 p.

Hart, J. L. 1930. The spawning and early life history of the whitefish, Coregonus clupeaformis (Mitchill), in Bay of Quinte, Ontario. Contributions to Canadian Biology and Fisheries, New Series. 6: 165-214.
Hart, J. L. 1931. The food of the whitefish Coregonus clupeaformis (Mitchill) in Ontario waters, with a note on the parasites. Contributions to Canadian Biology and Fisheries, New Series. 6: 445-454.

Hartman, G. F. 1957. Report on Iosegun Lake. Unpublished Report. Alberta Department of Lands and Forests. $7 \mathrm{p}$.

Harvey, H. H. 1955. An investigation into the parasites of the fishes of Lake Wellman, Duck Mountains. Manitoba Department of Mines \& Natural Resources, Games \& Fisheries Branch Report. 30 p.

Henderson, J. T. 1926. Description of a copepod gill-parasite of pike-perches in lakes of Northern Quebec, including an account of the free-swimming male and some developmental stages. Contributions to Canadian Biology and Fisheries, New Series. 3: 235-245.

Hicks, F. J. and Threlfall, W. 1973. Metazoan parasites of salmonids and coregonids from coastal Labrador. Journal of Fish Biology. 5: 399-415.

Hobbs, R. (undated). Helminths of whitefish (Coregonus clupeaformis) of Lesser Slave Lake with particular reference to Cotylurus erraticus metacercariae. Unpublished Report. Department of Zoology, University of Alberta, Edmonton, Alberta.

Hoffman, G. L. and Dunbar, C. E. 1961. Mortality of eastern brook trout caused by plerocercoids (Cestoda: Pseudophyllidea: Diphyllobothridae) in the heart and viscera. Journal of Parasitology. 47: 399-400. 
Holloway, J. A. and Curtis, M. A. 1982. A model relating to plerocercoid growth to temperature for Schistocephalus solidus in the three-spine stickleback, Gasterosteus aculeatus. Abstracts, $5^{\text {th }}$ International Congress of Parasitology. Molecular and Biochemical Parasitology, Supplement. p. 471.

Holmes, J. C. , Hobbs, R. P. and Leong, T. S. 1977. Populations in perspective: community organization and regulation of parasite populations, p. 209-245. In: G. W. Esch (ed.). Regulation of Parasite Populations. Academic Press, New York.

Hopkins, S. H. 1931. Studies on Crepidostomum. II. The "Crepidostomum laureatum" of A. R. Cooper. Journal of Parasitology. 18: 79-91.

Hopkins, S. H. 1934. The papillose Allocreadiidae. Illinois Biological Monographs. 13: 80p.

Hoskins, G. E., Bell, G. R. and Evelyn, T. P. T. 1976. The occurrence, distribution and significance of infectious diseases and neoplasms observed in fish in the Pacific Region up to the end of 1974. Canada Department of the Environment, Fisheries \& Marine Service, Research Development Technical Report. 609: 35 p.

Hoskins, G. E. and Hulstein, L. P. 1977. Annual Report of the Diagnostic Service of the Fisheries and Marine Service, Pacific Region for 1975. Canada Department of the Environment, Fisheries \& Marine Service. Research Development Technical Report. 707: $35 \mathrm{p}$.

Hunter, G. W. III and Bangham, R. V. 1933.
Studies on the fish parasites of Lake Erie. II. New Cestoda and Nematoda. Journal of Parasitology. 19: 304-311.

Jackin, P. M. 1947. Appendix No. 18. Experimental rearing of the tapeworm Diphyllobothrium latum, p. 27. In: K. H. Doan. Fisheries Research Board of Canada. Annual Report of the Central Fisheries Research Station for 1947, with Investigators' Summaries as Appendices.

Jansen, W. A. 1991. Seasonal prevalence, intensity of infestation, and distribution of glochidia of Anodonta grandis simpsoniana Lea on yellow perch Perca flavescens. Canadian Journal of Zoology. 69: 964-972.

Jansen, W. A. and Hanson, J. M. 1991. Estimates of the number of glochidia produced by clams (Anodonta grandis simpsonia Lea), attaching to yellow perch (Perca flavescens), and surviving to various ages in Narrow Lake, Alberta. Canadian Journal of Zoology. 69: 973-977.

Johnson, R. P. 1971. Limnology and fishery biology of Black Lake, northern Saskatchewan. Saskatchewan Department of Natural Resources, Fisheries \& Wildlife Branch, Fisheries Report. No. 9: 46 p.

Jones, S. R. M. and Woo, P. T. K. 1990. Redescription of the leech Desserobdella phalera (Graf, 1899) n. comb. (Rhynchobdella: Glossiphoniidae), with notes on its biology and occurrence on fishes. Canadian Journal of Zoology. 68: 1951-1955.

Jones, S. R. M. and Woo, P. T. K. 1992a. Vector specificity of Trypanosoma catostumi and its infectivity to freshwater fishes. Journal 
of Parasitology. 78: 87-92.

Jones, S. R. M. and Woo, P. T. K. 1992 b. Antigenic characterisation of cultured trypanosomes isolated from three species of fishes. Systematic Parasitology. 23: 43-50.

Kabata, Z. 1969. Revision of the genus Salmincola Wilson, 1915 (Copepoda: Lernaeopodidae). Journal of the Fisheries Research Board of Canada. 26: 2987-3041.

Kakonge, S. A. K. 1972. The ecology of some metazoan parasites of, and their effects on, small stream fishes and fry. $\mathrm{Ph}$. D. Dissertation. Department of Biology, University of Waterloo, Waterloo, Ontario. $161 \mathrm{p}$.

Kask, J. L. 1954. Central Fisheries Research Station, Winnepeg, Man., p. 65-73. In: J. L. Kask. Annual Report of the Fisheries Research Board of Canada for 1953.

Kask, J. L. 1955. Central Fisheries Research Station, Winnipeg, Man., p. 69-74. In: J. L. Kask. Annual Report of the Fisheries Research Board of Canada for 1954.

Kask, J. L. 1956. Biological Station, Winnipeg, Man., p. 65-71. In: J. L. Kask. Annual Report of the Fisheries Research Board of Canada for the year 1955.

Kask, J. L. 1957. Biological Station, Winnipeg, Man., p. 69-74. In: J. L. Kask. Annual Report of the Fisheries Research Board of Canada - 1956-1957 for the fiscal year ended March 31, 1957.

Kask, J. L. 1958. Biological Station, Winnipeg, Man., p. 77-86. In: J. L. Kask.
Annual Report of the Fisheries Research Board of Canada - 1957-1958 for the fiscal year ended March 31, 1958.

Kask, J. L. 1959. Biological Station, London, Ont., p. 77-84. In: J. L. Kask. Annual Report of the Fisheries Research Board of Canada 1958-1959 for the fiscal year ended March 31 , 1959.

Kask, J. L. 1960. Biological Station, London, Ont., p. 85-92. In: J. L. Kask. Annual Report of the Fisheries Research Board of Canada for the fiscal year ended March 31, 1960.

Kask, J. L. 1961. Biological Station, London, Ont., p. 71-82. In: J. L. Kask. Annual Report of the Fisheries Research Board of Canada for the fiscal year ended March 31, 1961.

Kask, J. L. 1962. Biological Station, London, Ont., p. 83-93. In: J. L. Kask. Annual Report of the Fisheries Research Board of Canada for the fiscal year ended March 31, 1962.

Kask, J. L. 1963. Biological Station, London, Ont., p. 65-73. In: J. L. Kask. Annual Report of the Fisheries Research Board of Canada for the fiscal year ended March 31, 1963.

Kayton, R. J., Kritsky, D. C. and Tobias, R. C. 1979. Rhabdochona catostomi sp. $\mathrm{n}$. (Nematoda: Rhabdochonidae) from the intestine of Castostomus spp. (Caatostomidae). Proceedings of the Helminthological Society of Washington. 46: 224-227.

Keleher, J. J. 1946. Appendix No. 12. Tullibee of Great Slave Lake, p. 24-25. In: K. H. Doan. Fisheries Research Board of Canada. Annual Report of the Central Fisheries Research 
Station for 1946, with Investigators' Summaries as Appendices.

Keleher, J. J. 1947. Appendix No. 15. Lake Winnipeg tullibee, p. 23-27. In: K. H. Doan. Fisheries Research Board of Canada. Annual Report of the Central Fisheries Station for 1947, with Investigators' Summaries as Appendices.

Keleher, J. J. 1949. Appendix No. 22. Lake Winnipeg cisco investigations, 1947-1949, p. 49-51. In: K. H. Doan. Fisheries Research Board of Canada. Annual Report of the Central Fisheries Research Station for 1949, with Investigators' Summaries as Appendices.

Keleher, J. J. 1950a. Growth, maturity and Triaenophorus parasitism in relation to taxonomy of Lake Winnipeg ciscoes (Leucichthys). M. A. Thesis. Department of Zoology, University of Toronto, Toronto, Ontario.

Keleher, J. J. 1950b. Growth, maturity and Triaenophorus parasitism in relation to taxonomy of Lake Winnipeg ciscos (Leucichthys). Fisheries Research Board of Canada, Manuscript Report (Biology). 509: 74 p.

Keleher, J. J. 1950c. Appendix No. 14. Growth, maturity and Triaenophorus parasitism in relation to taxonomy of Lake Winnipeg ciscoes, p. 40-47. In: K. H. Doan. Fisheries Research Board of Canada. Annual Report of the Central Fisheries Research Station for 1950, with Investigators' Summaries as Appendices.

Keleher, J. J. 1952a. Growth and Triaenophorus parasitism in relation to taxonomy of Lake Winnipeg ciscoes (Leucichthys). Journal of the Fisheries Research Board of Canada. 8: 469-478.

Keleher, J. J. 1952b. Appendix No. 19. Ciscoes collected from lakes Manitoba, Dauphin and Winnipegosis, p. 57-59. In: W. A. Kennedy. Fisheries Research Board of Canada. Annual Report of the Central Fisheries Research Station for 1952, with Investigators' Summaries as Appendices.

Keleher, J. J. 1953. Appendix No. 18. Summary of field work at Churchill, Manitoba and growth of the Churchill River cisco, Leucichthys sp., p. 48-49. In: W. A. Kennedy. Fisheries Research Board of Canada. Annual Report of the Central Fisheries Research Station for 1953, with Investigators' Summaries as Appendices.

Keleher, J. J. and Matheson, J. E. 1951. Appendix No. 15. Cisco research during summer of 1951, p. 35. In: K. H. Doan. Fisheries Research Board of Canada. Annual Report of the Central Fisheries Research Station for 1951, with Investigators' Summaries as Appendices.

Kennedy, M. J. 1979. The responses of miracidia and cerariae of Bunodera mediovitellata (Trematoda: Allocreadiidae) to light and to gravity. Canadian Journal of Zoology. 57: 603-609.

Kennedy, W. A. 1946. Appendix No. 6. Surveys of Tathlina and Kakisa lakes, N. W. T., p. 14. In: K. H. Doan. Fisheries Research Board of Canada. Annual Report of the Central Fisheries Research Station for 1946, with Investigators' Summaries as Appendices. 
Kennedy, W. A. 1948a. Appendix No. 6. Yellowknife whitefish, p. 16. In: K. H. Doan. Fisheries Research Board of Canada. Annual Report of the Central Fisheries Research Station for 1948, with Investigators' Summaries as Appendices.

Kennedy, W. A. 1948b. Appendix No. 7. Lake Winnipeg studies, p. 16-17. In: K. H. Doan. Fisheries Research Board of Canada. Annual Report of the Central Fisheries Research Station for 1948, with Investigators' Summaries as Appendices.

Kenuedy, W. A. 1949. Appendix No. 18. Nueltin Lake, p. 44-45. In: K. H. Doan. Fisheries Research Board of Canada. Annual Report of the Central Fisheries Station for 1949, with Investigators' Summaries as Appendices.

Kennedy, W. A. 1951. The bearing of the Great Slave Lake and Lake Winnipeg studies on the Triaenophorus problem. Fisheries Research Board of Canada, Manuscript Report (Biology). 516: 5 p.

Kennedy, W. A. 1955. Report for 1955 of the Central Fisheries Research Station, p. 1-8. In: W. A. Kennedy. Fisheries Research Board of Canada. Annual Report of the Central Fisheries Research Station for 1955.

Kennedy, W. A. 1958. Year End Summary Report 1958. Fisheries Research Board of Canada. Annual Report of the Biological Station and Technical Unit, London, Ontario. $3 \mathrm{p}$.

Kentiedy, W. A. and Sprules, W. M. 1967. Goldeye in Canada. Fisheries Research Board of Canada, Bulletin. 161: 45 p.
Kinnis, P. and Curtis, M. A. 1981. The occurrence of fish parasites in LG-2, a large, newly inundated reservoir in northern Quebec. Program \& Abstracts. 20 th Annual Meeting, Canadian Society of Zoologists. Bulletin of the Canadian Society of Zoologists. 12(2): 63.

Klasson, G. J., Beverley-Burton, M. and Dechtiar, A. O. 1985. Ligictaluridus posthon n. sp. (Monogenea: Ancyrocephalidae) from Noturus flavus Rafinesque (Siluriformes: Ictaluridae) in Ontario, Canada. Canadian Journal of Zoology. 63: 2071-2073.

Klemm, D. J. 1982. Leeches (Hirudinea) of North America. United States Environmental Protection Agency. Office of Research Development. EPA-600/3-82-025: 177 p.

Ko, R. C. and Anderson, R. C. 1969. A revision of the genus Cystidicola Fischer, 1798 (Nematoda: Spiruroidea) of the swim bladder of fishes. Journal of the Fisheries Research Board of Canada. 26: 849-864.

Kooyman, A. H. 1951. Speckled trout investigations, northern Manitoba, Nelson River area. Manitoba Department of Mines \& Natural Resources, Fisheries Branch Report. 4 p.

Koshinsky, G. D. 1965. Limnology and fisheries of five Precambrian headwater lakes near Lac la Ronge, Saskatchewan. Saskatchewan Department of Natural Resources, Fisheries Branch, Fisheries Report. No. 7: $51 \mathrm{p}$.

Kuitunen-Ekbaum, E. 1933. A study of the cestode genus Eubothrium of Nybelin in Canadian fishes. Contributions to Canadian Biology \& Fisheries, New Series. 8: 89-98. 
Kuitunen-Ekbaum, E. 1936. On the hatching of the eggs of the cestode Triaenophorus crassus. Fisheries Research Board of Canada, Manuscript Report (Biology). No. 302: 5 p.

Kuitunen-Ekbaum, E. 1937 a. Diphyllobothrium sp. plerocercoid in the lake trout from Algonquin Park, Ontario. Fisheries Research Board of Canada, Manuscript Report (Biology). No. 301: 3 p.

Kuitunen-Ekbaum, E. 1937b. Notes on the biology and partial life history of the dracunculid, Philonema oncorhynchi Kuitunen-Ekbaum. Fisheries Research Board of Canada, Manuscript Report (Biology). No. 304: 8 p.

Kussat, R. H. 1966. Bottom fauna studies in relation to the biology of certain fishes of the Bow River. M. Sc. Thesis. Department of Biology, University of Calgary, Calgary, Alberta. 76 p.

Kussat, R. H. A comparison of aquatic communities in the Bow River above and below sources of domestic and industrial wastes from the city of Calgary. Canadian Fish Culturist. 40: 3-31.

Lageux, R. 1966. Présence de larves plerocercoides de Diphyllobothrium (Cestode) sur la truite du Québec. Naturaliste Canadien. 93: $440-441$

Lankester, M. W. and Smith, J. D. 1980. Host specificity and distribution of the swimbladder nematodes, Cystidicola farionis Fischer, 1798 and C. cristivomeri White, 1941 (Habronematoidea), in salmonid fishes of Ontario. Canadian Journal of Zoology. 58: 1298-1305.
Lawler, G. H. 1950a. Appendix No. 7. Triaenophorus studies at Heming Lake, 1950, p. 24-28. In: K. H. Doan. Fisheries Research Board of Canada. Annual Report of the Central Fisheries Research Station for 1950, with Investigators' Summaries as Appendices.

Lawler, G. H. 1950b. Appendix No. 8. Notes on Triaenophorus nodulosus in Heming Lake, p. 28-30. In: K. H. Doan. Fisheries Research Board of Canada. Annual Report of the Central Fisheries Station for 1950, with Investigators' Summaries as Appendices.

Lawler, G. H. 1950c. Appendix No. 9. Troutperch as the second intermediate host of Triaenophorus stizostedionis in Heming Lake, p. 30-31. In: K. H. Doan. Fisheries Research Board of Canada. Annual Report of the Central Fisheries Research Station for 1950, with Investigators' Summaries as Appendices.

Lawler, G. H. 1951a. Appendix No. 1. Observations of Triaenophorus at Heming Lake and vicinity, 1951, p. 14-15. In: K. H. Doan. Fisheries Research Board of Canada. Annual Report of the Central Fisheries Research Station for 1951, with Investigators' Summaries as Appendices.

Lawler, G. H. 1951b. Appendix No. 5. The identification of Heming Lake ciscoes, and parasitism with Triaenophorus crassus, p. 2021. In: K. H. Doan. Fisheries Research Board of Canada. Annual Report of the Central Fisheries Research Station for 1951, with Investigators' Summaries as Appendices.

Lawler, G. H. 1951c. Appendix No. 3. Pike control and whitefish infestation, Heming Lake, p. 16-19. In: K. H. Doan. Fisheries Research Board of Canada. Annual Report of 
the Central Research Station for 1951, with Investigators' Summaries as Appendices.

Lawler, G. H. 1952a. A new North American host for the fish parasite Triaenophorus nodulosus (Pallas). Canadian Field-Naturalist. 66: 111.

Lawler, G. H. 1952b. Appendix No. 1. Host studies on Triaenophorus and the effect of Triaenophorus on growth, p. 23-25. In: W. A. Kennedy. Fisheries Research Board of Canada. Annual Report of the Central Fisheries Research Station for 1952, with Investigators' Summaries as Appendices.

Lawler, G. H. 1952c. Appendix No. 2. Pike exploitation and whitefish infection with Triaenophorus crassus at Heming Lake, p. 2526. In: W. A. Kennedy. Fisheries Research Board of Canada. Annual Report of the Central Fisheries Research Station for 1952, with Investigators' Summaries as Appendices.

Lawler, G. H. 1952d. Appendix No. 5. Cisco taxonomy and parasitism with Triaenophorus crassus, p. 31-32. In: W. A. Kennedy. Fisheries Research Board of Canada. Annual Report of the Central Fisheries Research Station in 1952, with Investigators' Summaries as Appendices

Lawler, G. H. 1953a. Age, growth, production and infection with Triaenophorus nodulosus of the yellow perch, Perca flavescens (Mitchill), of Manitoba. Fisheries Research Board of Canada. Manuscript Report (Biology). No. 521: 19 p.

Lawler, G. H. 1953b. Appendix No. 1. Observations on Triaenophorus parasites at Heming Lake in 1953, p. 22-23. In: W. A.
Kennedy. Fisheries Research Board of Canada. Annual Report of the Central Fisheries Research Station for 1953, with Investigators' Summaries as Appendices.

Lawler, G. H. 1953c. Appendix No. 3. Pike control and whitefish infection at Heming Lake, p. 27-29. In: W. A. Kennedy. Fisheries Research Board of Canada. Annual Report of the Central Fisheries Research Station for 1953, with Investigators' Summaries as Appendices.

Lawler, G. H. 1953d. Appendix No. 8. Studies on the age and fecundity of Heming Lake whitefish during the period 1945 to 1952 . In: W. A. Kennedy. Fisheries Research Board of Canada. Annual Report of the Central Fisheries Research Station for 1953, with Investigators' Summaries as Appendices.

Lawler, G. H. 1954a. Preliminary check-list of the fish parasites of Heming Lake, Manitoba. Fisheries Research Board of Canada. Manuscript Report (Biology). No. 576: 6 p.

Lawler, G. H. 1954b. Observations on the trout-perch Percopsis omiscomaycus (Walbaum), at Heming Lake, Manitoba. Journal of the Fisheries Research Board of Canada. 11: 1-4.

Lawler, G. H. 1954c. Appendix No. 1. Pike exploitation and its relation to whitefish infection with Triaenophorus crassus, p. 1921. In: W. A. Kennedy. Fisheries Research Board of Canada. Annual Report of the Central Fisheries Research Board for 1954, with Investigators' Summaries as Appendices.

Lawler, G. H. 1954d. Appendix No. 6. Some miscellaneous studies at Heming Lake, p. 26- 
28. In: W. A. Kennedy. Fisheries Research Board of Canada. Annual Report of the Central Fisheries Research Station for 1954, with Investigators' Summaries as Appendices.

Lawler, G. H. 1954e. Appendix No. 4. Some cestodes of Heming Lake fish, p. 24-25. In: W.

A. Kennedy. Fisheries Research Board of Canada. Annual Report of the Central Fisheries Research Station for 1954, with Investigators' Summaries as Appendices.

Lawler, G. H. 1955. Life history studies of Triaenophorus at Heming Lake, Manitoba. Part I. The life of $T$. crassus and T. nodulosus in the final host, Esox lucius, with special reference to changes in infection rate from year to year. Fisheries Research Board of Canada. Manuscript Report (Biology). No. 604: $17 \mathrm{p}$.

Lawler, G. H. 1956a. Life history of Triaenophorus at Heming Lake, Manitoba. Part III. Studies on eggs, coracidia and procercoids of Triaenophorus at Heming Lake. Fisheries Research Board of Canada. Manuscript Report (Biology). No. 623: 16 p.

Lawler, G. H. 1956b. Appendix No. 1. Relationship of pike exploitation to rate of Triaenophorus infection in whitefish in Heming Lake, p. 4-6. In: W. A. Kennedy. Fisheries Research Board of Canada. Report summarizing Investigations of the Biological Station, Winnipeg to March 31, 1956.

Lawler, G. H. 1956c. Appendix No. 3. Incidence of Triaenophorus in the northern pike, p. 8-10. In: W. A. Kennedy. Fisheries Research Board of Canada. Report summarizing Investigations of the Biological Station, Winnipeg to March 31, 1956.
Lawler, G. H. 1957a. Appendix No. 1. Pike control and the incidence of Triaenophorus crassus in whitefish, p. 22-25. In: G. H. Lawler. Fisheries Research Board of Canada. Biological Station, Winnipeg, Man. Annual Report and Investigators' Summaries for 1956 (covering the period December 31, 1955 to March 31, 1957).

Lawler, G. H. 1957b. Appendix No. 2. Incidence of Triaenophorus in the northern pike, p. 26-28. In: G. H. Lawler. Fisheries Research Board of Canada. Biological Station, Winnipeg, Man. Annual Report and Investigators' Summaries for 1956 (covering the period December 31, 1955 to March 31, 1957).

Lawler, G. H. 1958a. Appendix No. 2. Incidence of Triaenophorus in the northern pike in Heming Lake, p. 25-27. In: W. A. Kennedy. Fisheries Research Board of Canada. Annual Report of the Biological Station, London, Ontario for the period April 1, 1957 to March 31, 1958 with Investigators' Summaries as Appendices.

Lawler, G. H. 1958b. Appendix No. 3. Incidence of Triaenophorus crassus in whitefish in Heming Lake, p. 28-29. In: W. A. Kennedy. Fisheries Research Board of Canada. Annual Report of the Biological Station, London, Ontario for the period April 1, 1957 to March 31, 1958 with Investigators' Summaries as Appendices.

Lawler, G. H. 1959a. Appendix No. 2. Incidence of Triaenophorus in northern pike in Heming Lake, p. 25-26. In: W. A. Kennedy. Fisheries Research Board of Canada. Annual Report of the Biological Station, London, Ontario for the period April 1, 1958 to March 
31, 1959, with Investigators' Summaries as Appendices.

Lawler, G. H. 1959b. Appendix No. 3. Incidence of Triaenophorus crassus in whitefish, Heming Lake, p. 27-28. In: W. A. Kennedy. Fisheries Research Board of Canada. Annual Report of the Biological Station, London, Ontario for the period April 1, 1958 to March 31, 1959, with Investigators' Summaries as Appendices.

Lawler, G. H. 1959c. Appendix No. 11. The occurrence of "black-spots" on Heming Lake fish, p. 35. In: W. A. Kennedy. Fisheries Research Board of Canada. Annual Report of the Biological Station, London, Ontario for the period April 1, 1958 to March 31, 1959, with Investigators' Summaries as Appendices.

Lawler, G. H. 1959d. Biology and control of the pike-whitefish parasitic worm, Triaenophorus crassus, in Canada. Fisheries Research Board of Canada. Progress Reports, London Biological Station \& Technical Unit. Progress Report. 1: 31-37.

Lawler, G. H. 1960a. Appendix No. 2. Incidence of Triaenophorus in the northern pike in Heming Lake, p. 22-24. In: W. A. Kennedy. Fisheries Research Board of Canada. Annual Report of the Biological Station, London, Ontario for the period April 1, 1959 to March 31, 1960, with Investigators' Summaries as Appendices.

Lawler, G. H. 1960b. Appendix No. 3. Incidence of Triaenophorus crassus in Heming Lake whitefish, p. 24-26. In: W. A. Kennedy. Fisheries Research Board of Canada. Annual Report of the Biological Station, London, Ontario for the period April 1, 1959 to March
31,1960 , with Investigators' Summaries as Appendices.

Lawler, G. H. 1961a. Heming Lake experiment. Fisheries Research Board of Canada, London Biological Station \& Technical Unit. Progress Report. 2: 48-50.

Lawler, G. H. 1961b. Appendix No. 2. Incidence of Triaenophorus in northern pike in Heming Lake, p. 19-20. In: W. A. Kennedy. Fisheries Research Board of Canada. Annual Report of the Biological Station, London, Ontario for the period April 1, 1960 to March 31,1961 , with Investigators' Summaries as Appendices.

Lawler, G. H. 1961c. Appendix No. 3. Incidence of Triaenophorus crassus in Heming Lake whitefish, p. 21-22. In: W. A. Kennedy. Fisheries Research Board of Canada. Annual Report of the Biological Station, London, Ontario for the period April 1, 1960 to March 31, 1961, with Investigators' Summaries as Appendices.

Lawler, G. H. 1961c. Appendix No. 5. Ectoand endoparasitic observations on Heming Lake, p. 23. In: W. A. Kennedy. Fisheries Research Board of Canada. Annual Report of the Biological Station, London, Ontario for the period April 1, 1960 to March 31, 1961, with Investigators' Summaries as Appendices.

Lawler, G. H. 1962. Appendix No. 8. "Blackspot" disease on Heming Lake fish, p. 28-29. In: W. A. Kennedy. Fisheries Research Board of Canada. Annual Report of the Biological Station, London, Ontario for the period April 1, 1961 to March 31, 1962, with Investigators' Summaries as Appendices. 
Lawler, G. H. 1963a. Appendix No. 5. Triaenophorus parasites in pike and coregonines, p. 24-25. In: W. A. Kennedy. Fisheries Research Board of Canada. Annual Report of the Biological Station, London, Ontario for the period April 1, 1962 to March 31,1963 , with Investigators' Summaries as Appendices.

Lawler, G. H. 1963b. Appendix No. 6. Occurrence of "black-spot" disease on Heming Lake fish, p. 26. In: W. A. Kennedy. Fisheries Research Board of Canada. Annual Report of the Biological Station, London, Ontario for the period April 1, 1962 to March 31, 1963, with Investigators' Summaries as Appendices.

Lawler, G. H. 1964a. Appendix No. 2. Triaenophorus parasites in pike and coregonines, p. 21-22. In: W. A. Kennedy. Fisheries Research Board of Canada. Annual Report of the Biological Station, London, Ontario for the period April 1, 1963 to March 31, 1964, with Investigators' Summaries as Appendices.

Lawler, G. H. 1964b. Appendix No. 4. "Blackspot" disease on Heming Lake fish, p. 23. In: W. A. Kennedy. Fisheries Research Board of Canada. Annual Report of the Biological Station, London, Ontario for the period April 1, 1963 to March 31, 1964, with Investigators' Summaries as Appendices.

Lawler, G. H. 1964c. Incidence of Ligula intestinalis in Heming Lake fish. Journal of the Fisheries Research Board of Canada. 21: 549554

Lawler, G. H. 1965a. Whitefish improvement pike control. Fisheries Research Board of Canada. London Biological Station \& Techical
Unit. Circular No. 7: 9-15.

Lawler, G. H. 1965b. Appendix No. 15. Whitefish improvement experiment - pike control phase, p. 38. In: W. A. Kennedy. Fisheries Research Board of Canada. Annual Report of the Biological Station, London, Ontario for the period April 1, 1964 to March 31,1965 , with Investigators' Summaries as Appendices.

Lawler, G. H. 1965c. Appendix No. 14. Triaenophorus parasites in pike and coregonines, p. 34-35. In: W. A. Kennedy. Fisheries Research Board of Canada. Annual Report of the Biological Station, London, Ontario for the period April 1, 1964 to March 31,1965 , with Investigators' Summaries as Appendices.

Lawler, G. H. 1965d. The food of pike, Esox lucius, in Heming Lake, Manitoba. Journal of the Fisheries Research Board of Canada. 22: 1357-1377.

Lawler, G. H. 1965e. Appendix No. 12. Incidence of "black-spot" disease in Heming Lake fish, p. 32-33. In: W. A. Kennedy. Fisheries Research Board of Canada. Annual Report of the Biological Station, London, Ontario for the period April 1, 1964 to March 31,1965 , with Investigators' Summaries as Appendices.

Lawler, G. H. 1965f. Appendix No. 13. Incidence of Ligula intestinalis, p. 33. In: W. A. Kennedy. Fisheries Research Board of Canada. Annual Report of the Biological Station, London, Ontario for the period April 1, 1964 to March 31, 1965, with Investigators' Summaries as Appendices. 
Lawler, G. H. 1966a. Appendix No. 8. Triaenophorus infection in pike and coregonines, p. 22-24. In: W. A. Kennedy. Fisheries Research Board of Canada. Annual Report of the Biological Station, London, Ontario for the period April 1, 1965 to March 31, 1966 with Investigators' Summaries as Appendices.

Lawler, G. H. 1966b. Appendix No. 6. "Blackspot" in Heming Lake fish, p. 21. In: W. A. Kennedy. Fisheries Research Board of Canada. Annual Report of the Biological Station, London, Ontario for the period April 1, 1965 to March 31, 1966, with Investigators' Summaries as Appendices.

Lawler, G. H. 1966c. Appendix No. 8. Ligula intestinalis in white sucker and spottail shiner, p. 24. In: W. A. Kennedy. Fisheries Research Board of Canada. Annual Report of the Biological Station, London, Ontario for the period April 1, 1965 to March 31, 1966, with Investigators' Summaries as Appendices.

Lawler, G. H. 1968. Triaenophorus nodulosus in burbot, Lota lota, from Heming Lake, Manitoba. Journal of the Fisheries Research Board of Canada. 25: 2523-2524.

Lawler, G. H. 1969. Aspects of the biology of Triaenophorus nodulosus in yellow perch, Perca flavescens in Heming Lake, Manitoba. Journal of the Fisheries Research Board of Canada. 26: 821-831.

Lawler, G. H. 1970. Parasites of coregonid fishes, p. 279-309. In: C. C. Lindsay and C. S. Woods (ed.). Biology of Coregonid Fishes. University of Manitoba Press, Winnipeg, Manitoba.
Lawler, G. H. and McBurney, A. B. 1953. Appendix No. 2. Laboratory and field studies of eggs, coracidia and procercoids of Triaenophorus at Heming Lake, Manitoba, 1953. In: W. A. Kennedy. Fisheries Research Board of Canada. Annual Report of the Central Fisheries Research Station for 1953.

Lawler, G. H. and Scott, W. B. 1954. Notes on the geographical distribution and hosts of the cestode genus Triaenophorus in North America. Journal of the Fisheries Research Board of Canada. 11: 884-893.

Lawler, G. H. and Watson, N. H. F. 1958. Limnological studies of Heming Lake, Manitoba and two adjacent lakes. Journal of the Fisheries Research Board of Canada. 15: 203-218.

Leong, R. T. S. 1975. Metazoan parasites of fishes of Cold Lake, Alberta: a community analysis. $\mathrm{Ph}$. D. Dissertation. Department of Zoology, University of Alberta, Edmonton, Alberta. 170 p.

Leong, T. S. and Holmes, J. C. 1974a Acquisition of helminths by coho salmon, Oncorhynchus kisutch, introduced into Cold Lake, Alberta: a comparison with helminths of native salmonid fish. Proceedings of the $3^{\text {rd }}$ International Congress of Parasitology. 3: 1639.

Leong, T. S. and Holmes, J. C. $1974 \mathrm{~b}$. Analysis of the circulation of Metechinorhynchus salmonis in Cold Lake, Alberta. Program \& Abstracts. $49^{\text {th }}$ Annual Meeting, American Society of Parasitologists. p. 44-45.

Leong, T. S. and Holmes, J. C. 1981. 
Communities of metazoan parasites in open water fishes of Cold Lake, Alberta. Journal of Fish Biology. 18: 693-713.

Lester, R. J. G. 1971a. Method of attachment of Gyrodactylus to Gasterosteus and its rejection by the sloughing of mucus. Program \& Abstracts. 46 ${ }^{\text {th }}$ Annual Meeting, American Society of Parasitologists. p. 24.

Lester, R. J. G. 1971b. The influence of Schistocephalus plerocercoids on the respiration of Gasterosteus and a possible resulting effect on the behaviour of the fish. Canadian Journal of Zoology. 49: 361-366.

Lester, R. J. G. 1972. Attachment of Gyrodactylus to Gasterosteus and host response. Journal of Parasitology. 58: 717722.

Lester, R. J. G. 1975. Parasites of Gasterosteus aculaetus near Vancouver, British Columbia. Syesis. 7: 195-200.

Lester, R. J. G. 1977. An estimate of the mortality in a population of Perca flavescens owing to the trematode Diplostomum adamsi. Canadian Journal of Zoology. 55: 288-292.

Lester, R. J. G. and Adams, J. R. 1974a. Gyrodactylus alexanderi: reproduction, mortality, and effect on its host Gasterosteus aculeatus. Canadian Journal of Zoology. 52: 827-833.

Lester, R. J. G. and Adams, J. R. 1974b. A simple model of a Gyrodactylus population. International Journal for Parasitology. 4: 497506.

Lester, R. J. G. and Daniels, B. A. 1976. The eosinophilic cell of the white sucker, Catostomus commersoni. Journal of the Fisheries Research Board of Canada. 33: 139144.

Lester, R. J. G. and Huizinga, H. W. 1977. Diplostomum adamsi sp. n.: description, life cycle, and pathogenesis in the retina of Perca flavescens. Canadian Journal of Zoology. 55: 64-73.

Lester, R. J. G. and Lee, T. D. G. 1976. Infectivity of the progenetic metacercariae of Diplostomum spathaceum. Journal of Parasitology. 62: 832-833.

Li, M.-M. and Arai, H. P. 1988. Electron microscopical observations on the effects of 30 sera from two species of Catostomus on the tegument of Hunterella nodulosa (Cestoidea: Caryophyllidae). Canadian Journal of Zoology. 66: 1191-1196.

Li, M.-M. and Arai, H. P. 1991. On the ultrastructure of the scolex tegument, organelles and sensory receptors of Glaridacris catostomi Cooper (Cestoda: Caryophyllidae). (Ch, en). Acta Zoologica Sinica. 37: 113-122.

Libin, M. L. 1951a. Appendix No. 12. Observations on the effect of pituitary suspensions and extracts on $T$. crassus and its host, E. lucius, p. 31. In: K. H. Doan. Fisheries Research Board of Canada. Annual Report of the Central Fisheries Research Station for 1951, with Investigators' Summaries as Appendices.

Libin, M. L. 1951b. Laboratory experiments on the control of the tapeworm Triaenophorus crassus. Fisheries Research Board of Canada. 
Manuscript Report (Biology). No. 493: 82 p.

Libin, M. L. 1951c. Laboratory experiments on the control of the tapeworm, Triaenophorus crassus. M. Sc. Thesis. Department of Zoology, University of Alberta, Edmonton, Alberta.

Libin, M. L. 1952. Appendix No. 17. Studies on Triaenophorus crassus Forel at South Bay, Ontario, p. 50-55. In: W. A. Kennedy. Fisheries Research Board of Canada. Annual Report of the Central Fisheries Research Station for 1952, with Investigators' Summaries as Appendices.

Libin, M. L. 1953a. Appendix No. 16. Laboratory infection of Esox masquinongy with Triaenophorus crassus, p. 47. In: W. A. Kennedy. Fisheries Research Board of Canada. Annual Report of the Central Fisheries Research Station for 1953, with Investigators' Summaries as Appendices.

Libin, M. L. 1953b. Appendix No. 17. Laboratory infection of trout with Triaenophorus crassus, p. 48. In: W. A. Kennedy. Fisheries Research Board of Canada. Annual Report of the Central Fisheries Research Station for 1953, with Investigators' Summaries as Appendices.

Little, S. W. 1954. A survey of the cestode genera Proteocephalus and Bothriocephalus from freshwater fish of western Canada, including a re-description of Proteocephalus luciopercae (Wardle) and Proteocephalus stizostethi (Hunter and Bangham). M. Sc. Thesis. Department of Zoology, University of Manitoba, Winnipeg, Manitoba. 56 p.

Lubinsky, G. A. and Loch, J. S. 1979.
Ichthyoparasites of Manitoba: literature review and bibliography. Canada Department of Fisheries \& Oceans, Fisheries Service. Manuscript Report. No. 113: 29 p.

Lyster, L. L. 1939. Parasites of freshwater fish. I. Internal trematodes of commercial fish in the central St. Lawrence watershed. Canadian Journal of Research, Section D. 17: 254-268.

Lyster, L. L. 1940a. Parasites of freshwater fish. II. Parasitism of speckled and lake trout and fish found associated with them in Lake Commandant, Que. Canadian Journal of Research, Section D. 18: 66-78.

Lyster, L. L. 1940b. Apophallus imperator sp. nov., a heterophyid encysted in trout, with a contribution to its life history. Canadian Journal of Research, Section D. 18: 106-121.

MacCallum, W. G. 1895. On the anatomy of two distome parasites of freshwater fish. Veterinary Magazine. 2: 401-412.

Mackie, G. L., Morton, W. B. and Ferguson, M. S. 1983. Fish parasitism in a new impoundment and differences upstream and downstream. Hydobiologica. 99: 197-206.

Mackiewicz, J. S. 1963. Monobothrium hunteri sp. n. (Cestoidea: Caryophyllidea) from Catostomus commersoni (Lacépède) (Pisces: Catostomidae) in North America. Journal of Parasitology. 49: 723-730.

Mackiewicz, J. S. 1974. The genus Caryophyllaeus Gmelin (Cestoidea: Caryophyllidae) in the Nearctic. Proceedings of the Helminthological Society of Washington. 41: 184-191. 
Mackiewicz, J. S. 1976. Glaridacris vogei n. sp. (Cestoidea: Caryophyllidea) from catostomid fishes in western North America. Transactions of the American Microscopical Society. 95: 92-97.

Mackiewicz, J. S. and McCrae, R. C. 1962. Hunterella nodulosa gen. n., sp. n. (Cestoidea: Caryophyllaeidae) from Catostomus commersoni (Lacépède) ( $\mathrm{P}$ isces: Catostomidae). Journal of Parasitology. 48: 798-806.

MacKinnon, B. M. and Burt, M. D. B. 1982. The comparative ultrastructure of sperm from Bothrimonas sturionis (Pseudophyllidae), Pseudanthobothrium sp. (Tetraphyllidea) and Monoecocestus americamus(Cyclophyllidea). Parasitology 85(2): xxxi.

MacLulich, D. A. 1943a. Proteocephalus parallacticus, a new species of tapeworm from lake trout, Cristivomer namaycush. Canadian Journal of Research, Section D. 21: 145-149.

MacLulich, D. A. 1943b. Parasites of trout in Algonquin Park, Ontario. Canadian Journal of Research, Section D. 21: 405-412.

Madill, J. 1988. New Canadian records of leeches (Annelida: Hirudinea) parasitic on fish. Canadian Field-Naturalist. 102: 658-688.

Magath, T. B. 1928. Distribution of the broad tapeworm. Journal of the American Medical Association. 90: 1650.

Magath, T. B. and Essex, H. E. 1931. Concerning the distribution of Diphyllobothrium latum in North America. Journal of Preventative Medicine. 5: 227-243.
Mahon, R. 1976. Effects of the cestode Ligula intestinalis on spottail shiners, Notropis hudsonius. Canadian Journal of Zoology. 54: 2227-2229.

Manzer, J. I. 1976. Distribution, food, and feeding of the threespine stickleback, Gasterosteus aculeatus, in Great Central Lake, Vancouver Island, with comments on competition for food with juvenile sockeye salmon, Oncorhynchus nerka. Fishery Bulletin. 75: 647-668.

Marcogliese, D. J. 1992. Metazoan parasites of sticklebacks on Sable Island, northwest Atlantic Ocean: biogeographic considerations. Journal of Fish Biology. 41: 399-407.

Marcogliese, D. J. 1995. Comparison of parasites of mummichogs and sticklebacks from brackish and freshwater ponds on Sable Island, Nova Scotia. American Midland Naturalist. 133: 333-343.

Marcogliese, D. J. and Cone, D. K. 1991a. Do brook trout (Salvelinus fontinalis) from insular Newfoundland have different parasites than their mainland counterparts? Canadian Journal of Zoology. 69: 809-811.

Marcogliese, D. J. and Cone, D. K. 1991 b. Importance of lake characteristics in structuring parasite communities of salmonids from insular Newfoundland. Canadian Journal of Zoology. 69: 2962-2967.

Margolis, L. 1964. Paurorhynchus hiodontis Dickerman, 1954 (Trematoda: Bucephalidae): a second record involving a new host and locality in Canada. Canadian Journal of Zoology. 42: 716. 
Margolis, L. 1966. The swim bladder nematodes of Pacific salmon (genus Oncorhynchus). Proceedings of the $1^{\text {st }}$ International Congress of Parasitology. p. 559560 .

Margolis, L. 1967a. The swim bladder nematodes (Cystidicolinae) of Pacific salmon (genus Oncorhynchus). Canadian Journal of Zoology. 45: 1183-1199.

Margolis, L. 1967b. Blood feeding in Salvelinema walkeri (Nematoda: Cystidicolinae), a parasite of coho salmon (Oncorhynchus kisutch). Canadian Journal of Zoology. 45: 1295-1296.

Margolis, L. \& Kabata, Z. 1967. The structure of the buccal region of Salvelinema Trofimenko, 1962 (Nematoda: Cystidicolinae). Canadian Journal of Zoology. 45: 1067-1072.

Margolis, L. and Moravec, F. 1982. Ramellogammarus vancouverensis Bousfield (Amphipoda) as an intermediate host for salmonid parasites in British Columbia. Canadian Journal of Zoology. 60: 1100-1104.

Margolis, L., Moravec, F. and McDonald, T. E. 1975. Rhabdochona kisutchi sp. nov. (Nematoda: Spiruroidea) from coho salmon, Oncorhynchus kisutch (Walbaum) of western Canada. Canadian Journal of Zoology. 53: 960-966.

Markevich, A. P. 1956. New representatives of Copepoda Parastica of the family Ergasilidae. (Uk, ru, en). Pratsi NaukovaDoslidnogo Institutu Biologii, Kiivskii Derzhavnii Universitet. 4: 107-123.

Markevich, A. P. 1956. Parasitic copepods of fishes of the USSR. (Ru). Izdatel'stvo Akademii Nauk Ukrainskoi SSR, Kiev. 259 p.

McAllister, D. J. and Mudry, D. R. 1983. Diseases and parasites of Alberta fishes and potential consequences of their interbasin transfer. Prepared for Alberta Environmental Trust by Techman Inc., Calgary, Alberta. 80 p.

McBurney, A. B. and Lawler, G. H. 1953. Plankton studies in relation to Triaenophorus in Heming Lake, Manitoba. Fisheries Research Board of Canada. Manuscript Report (Biology). No. 546: 15 p.

McCart, P., Tripp, D. and Withler, R. 1982. Spawning and distribution of lake whitefish (Coregonus clupeaformis) in Athabasca River and Lake Athabasca. Prepared for Alberta Environment by Aquatic Environments Ltd. AEL 4015: $38 \mathrm{p}$.

McLennan, D. A. and Shires, V. L. 1995. Correlation between the level of infection with Bunodera inconstans and Neoechinrhynchus rutili and behavioral intensity in female brook sticklebacks. Journal of Parasitology. 81: 675682.

McLeod, J. A. 1943. Preliminary investigations of eight lakes in the Whiteshell Forest Reserve, Manitoba. Manitoba Department of Mines \& Natural Resources. Game \& Fisheries Branch Report, Sections AH: $107 \mathrm{p}$.

McLeod, J. A. 1944. Biological Investigation of Lake Wellman. Manitoba Department of Mines \& Natural Resources. Game \& Fisheries Branch Report.

McLeod, J.A. and Moir, D. R. 1944. An 
investigation of certain waters in the Duck Mountains: investigations of Lake Wellman. Section "A" - General preamble; Section "B" Wellman Lake. Summers 1942 \& 1944. Manitoba Department of Mines \& Natural Resources, Game \& Fisheries Branch Report, $15 \mathrm{p}$.

McMurtie, G. E. and Carter, N. M. 1948. The inspection of whitefish flesh for parasitic worms. Fisheries Research Board of Canada. Progress Report of the Pacific Coast Stations. No. 77: 95-97.

McPhail, J. D. and Peacock, S. D. 1983. Some aspects of the cestode (Schistocephalus solidus) on reproduction in the threespine stickleback (Gasterosteus aculeatus): evolutionary aspects of the host-parasite interactions. Canadian Journal of Zoology. 61: 901-908.

McTavish, W. B. 1952. Biological investigations of Southern Indian Lake Summer 1952. Manitoba Department of Mines \& Natural Resources, Fisheries Branch Report. 97 p.

McTavish, W. B. 1953. A biological investigation of Groschak Lake, Manitoba June 1953. Manitoba Department of Mines \& Natural Resources, Fisheries Branch Report. $11 \mathrm{p}$.

Measures, L. N. 1987. Intermediate hosts and epizootiology of Eustrongylides tubifex (Nematoda: Dioctophymatoidea) in an eutrophic ecosystem. Program \& Abstracts. $62^{\text {nd }}$ Annual Meeting, American Society of Parasitologists. p. 21.

Measures, L. N. 1988a. Revision of the genus
Eustrongylides Jagerskiöld, 1909 (Nematoda: Dioctyophymoidea) of piscivorous birds. Canadian Journal of Zoology. 66: 885-895.

Measures, L. N. 1988b. Epizootiology, pathology, and description of Eustrongylides tubifex (Nematoda: Dioctophymoidea) in fish. Canadian Journal of Zoology. 66: 2212-2222.

Meyer, M. C. 1946. Further notes on the leeches (Piscicolidae) living on freshwater fishes of North America. Transactions of the American Microscopical Society. 65: 237-249.

Meyer, M. C. and Moore, J. P. 1954. Notes on Canadian leeches (Hirudinea), with description of a new species. Wasmann Journal of Biology. 12: 63-96.

Meyer, M. C. and Roberts, L. S. 1977. Cystobranchus mammilatus (Malm), a piscicolid leech new to North America. In: Excerta Parasitologica en Memoria del Doctor Eduardo Caballero y Cahallero. Universidad Nacional Autonoma de Mexico. Instituto de Biologia, Publicaciones Especiales. 4: 513517.

Miller, M. J. 1936. Bunodera eucaliae gen. et sp. nov., a new papillose Allocreadiidae from the stickleback. Canadian Journal of Research, Section D. 14: 11-14.

Miller, M. J. 1940a. Black spot in fishes. Canadian Journal of Comparative Medicine. 4: 303-305.

Miller, M. J. 1940b. Parasites of freshwater fish. III. Further studies on the internal trematodes of fish in the central St. Lawrence watershed. Canadian Journal of Research, Section D. 18: 423-434. 
Miller, M. J. 1941. The life history of Apophallus brevis Ransom, 1920. Journal of Parasitology. 27(Suppl.): 12.

Miller, M. J. 1942. Black spot disease of speckled trout. Revue Canadienne de Biologie.

1: 464-471.

Miller, R. B. 1943. Studies on the cestodes of the genus Triaenophorus from fish of Lesser Slave Lake, Alberta. I. Introduction and life of Triaenophorus crassus Forel and T. nodulosus (Pallas) in the definitive host, Esox lucius. Canadian Journal of Research, Section D. 21: 160-170.

Miller, R. B. 1944a. Suggestions for experiments in the control of the pikewhitefish tapeworm, Triaenophorus crassus. Alberta Department of Lands \& Mines, Fisheries Branch Report. 15 p.

Miller, R. B. 1944b. Fish parasites collected at Great Slave Lake in 1954. Manuscript Report. Department of Zoology, University of Alberta, Edmonton, Alberta. 4 p.

Miller, R. B. 1944c. Appendix No. 4. The candling of fillets, p. 12-13. In: K. H. Doan. Fisheries Research Board of Canada. Annual Report of the Central Fisheries Research Station for 1944, with Investigators' Summaries as Appendices.

Miller, R. B. 1944d. Appendix No. 5. Life cycle of Triaenophorus nodulosus and Triaenophorus crassus, p. 13-14. In: K. H. Doan. Fisheries Research Board of Canada. Annual Report of the Central Fisheries Research Station for 1944, with Investigators' Summaries as Appendices.
Miller, R. B. 1945a. Effect of Triaenophorus on growth of two fishes. Journal of the Fisheries Research Board of Canada. 6: 334337.

Miller, R. B. 1945b. Studies on the cestodes of the genus Triaenophorus from fish of Lesser Slave Lake, Alberta. V. Description and life history of Triaenophorus stizostedionis $\mathrm{n}$. $\mathrm{sp}$. Canadian Journal of Research, Section D. 23: 117-127.

Miller, R. B. 1945c. Studies on the cestodes of the genus Triaenophorus from fish of Lesser Slave Lake, Alberta. IV. The life of Triaenophorus crassus Forel in the second intermediate host. Canadian Journal of Research, Section D. 23: 105-115.

Miller, R. B. 1945d. Parasites collected from fishes of Lake Athabasca and Great Slave Lake. Unpublished report. Department of Zoology, University of Alberta, Edmonton, Alberta. 9 p.

Miller, R. B. 1945e. Studies on the cestodes of the genus Triaenophorus from fish of Lesser Slave Lake, Alberta. III. Notes on Triaenophorus nodulosus (Pallas) in the second intermediate host. Canadian Journal of Research, Section D. 23: 1-5.

Miller, R. B. 1946a. Notes on the Arctic grayling, Thymallus signifer Richardson, from Great Bear Lake. Copeia. 4: 227-236.

Miller, R. B. 1946b. Cestode "parasitized" by acanthocephalan. Science. 103: 762.

Miller, R. B. 1946c. The life history of the pike-whitefish tapeworm, Triaenophorus crassus. Alberta Department of Lands \& 
Mines, Fisheries Branch Report. $15 \mathrm{p}$.

Miller, R. B. 1946d. Appendix VI. Reports for 1945 on the Northwestern Fisheries Investigations. C. Great Bear Lake Investigations, p. 44. In: A. T. Cameron. Annual Report of the Fisheries Research Board of Canada for the year 1945.

Miller, R. B. 1947. Chapter IV. Great Bear Lake, p. 31-44. In: Northwest Canadian Fisheries Surveys in 1944-1945. Fisheries Research Board of Canada. Bulletin No. 72.

Miller, R. B. 1948. Reduction of Triaenophorus infestation in whitefish by depletion of the cisco population. Canadian Journal of Research, Section D. 26: 67-72.

Miller, R. B. 1950. The Square Lake experiment: an attempt to control Triaenophorus crassus by poisoning pike. Canadian Fish Culturist. 6: 3-18.

Miller, R. B. 1952a. A review of the Triaenophorus problem in Canadian lakes. Fisheries Research Board of Canada. Bulletin No. 85: 45 p.

Miller, R. B. 1952b. Appendix No. 16. The Square Lake poisoning experiment, p. 49-50. In: W. A. Kennedy. Fisheries Research Board of Canada. Annual Report of the Central Fisheries Research Station for 1952, with Investigators' Summaries as Appendices.

Miller, R. B. 1953. Appendix No. 14 Triaenophorus research in Alberta, 1953, p. 41-46. In: W. A. Kennedy. Fisheries Research Board of Canada. Annual Report of the Central Fisheries Research Station for 1953, with Investigators' Summaries as Appendices.
Miller, R. B. 1954. Tapeworm infection in Lesser Slave Lake. Progressive Fish Culturist. 16: 184.

Miller, R. B. and Huston, M. J. 1952. Effect of certain chemicals on the eggs and coracidia of Triaenophorus crassus. Unpublished Address. Science Association, University of Alberta, Edmonton, Alberta.

Miller, R. B. and Johnson, R. P. 1952. Appendix No. 15. Pike, cisco, Cyclops interrelationships in Square Lake, p. 44-49. In: W. A. Kennedy. Fisheries Research Board of Canada. Annual Report of the Central Fisheries Research Station for 1952, with Investigators' Summaries as Appendices.

Miller, R. B. and Kennedy, W. A. 1948. Observations of the lake trout of Great Bear Lake. Journal of the Fisheries Research Board of Canada. 7: 176-189.

Miller, R. B. and Macdonald, W. H. 1950. Preliminary biological surveys of Alberta watersheds, 1947-1949. Alberta Department of Lands \& Forests. Fish and Game Branch Report. 139 p.

Miller, R. B. and Watkins, H. B. 1946. An experiment on the control of the cestode, Triaenophorus crassus Forel. Canadian Journal of Research, Section D. 24: 175-179.

Mizelle, J. D. and Donahue, M. A. 1944. Studies on monogenetic trematodes. XI. Dactylogyridae from Algonquin Park fishes. American Midland Naturalist. 31: 600-624.

Molnar, K., Chan, G.-L. and Fernando, C. H. 1982. Some remarks on the occurrence and development of philometrid nematodes 
infecting the white sucker, Catostomus commersoni Lacépède(Pisces: Catostomidae), in Ontario. Canadian Journal of Zoology. 60: 443-451.

Molnar, K. and Fernando, C. H. 1975a. Morphology and development of Philometra cylindracea (Ward and Magath, 1916) (Nematoda: Philometridae). Journal of Helminthology. 49: 19-24.

Molnar, K. and Fernando, C. H. 1975 b. Philometra kobuleji sp. n. (Nematoda: Philometridae). Journal of Helminthology. 49: 101-105.

Molnar, K., Hanek, G. and Fernando, C. H. 1974. Parasites of fishes of Laurel Creek, Ontario. Journal of Fish Biology. 6: 717-728.

Monaco, L. and Mizelle, J. D. 1955. Studies on monogenetic trematodes. XVII. The genus Dactylogyrus. American Midland Naturalist. 53: 455-477.

Moore, J. E. 1964. Notes on leeches (Hirudinea) of Alberta. National Museums of Canada. Natural History Papers. 27: 1-15.

Moore, J. P. 1924. The leeches (Hirudinea) of Lake Nipigon. Ontario Fisheries Research Laboratory, Publication 23: 14 p. (University of Toronto, Studies in Biology. 25: 17-31).

Moore, J. P. 1936. The leeches of Lake Nipissing. Canadian Field-Naturalist. 50:112114.

Moore, J. P. and Meyer, M. C. 1951. Leeches (Hirudinea) from Alaska and adjacent waters. Wasmann Journal of Biology. 9: 11-?7.
Moravec, F. 1980. Notes on the morphology of Capillaria catostomi Pearse, 1924. Folia Parasitologia (Praha). 27: 381-382.

Moravec, F and Arai, H. P. 1971. The North and Central American species of Rhabdochona Railliet, 1916 (Nematoda: Rhabdochonidae) of fishes, including Rhabdochona canadensis sp. nov. Journal of the Fisheries Research Board of Canada. 28: 1645-1662.

Morris, G. P. and Finnegan, C. V. 1968. Studies on the differentiating plerocercoid cuticle of Schistocephalus solidus. I. The histochemical analysis of cuticle development. Canadian Journal of Zoology. 46: 115-121.

Morris, G. P. and Finnegan, C. V. 1969. Studies cn the differentiating plerocercoid cuticle of Schistocephalus solidus. II. The ultrastructural examination of cuticle development. Canadian Journal of Zoology. 47: 957-964.

Moulton, J. M. 1931. A new species of Haplonema Ward et Magath, 1916 from the stomach of Lota maculosa. Journal of Parasitology. 18: 105-107.

Mudry, D. R. 1972. Aspects of the biology of Hunterella nodulosa Mackiewicz and McCrae, 1962 (Cestoidea: Caryophyllidae). Ph. D. Dissertation. Department of Biology, University of Calgary, Calgary, Alberta. 138 p.

Mudry, D. R. and Anderson, R. S. 1977. Helminth and arthropod parasites of fishes in the mountain national parks of Canada. Journal of Fish Biology. 11: 21-33.

Mudry, D. R. and Arai, H. P. 1973a. Population dynamics of Hunterella nodulosa 
(Cestoidea: Caryophyllidae) in Alberta. Canadian Journal of Zoology. 51: 787-792.

Mudry, D. R. and Arai, H. P. 1973b. The life cycle of Hunterella nodulosa Mackiewicz and McCrae, 1962 (Cestoidea: Caryophyllidae) in Alberta. Canadian Journal of Zoology. 51: 781-786.

Mudry, D. R. and McCart, P. 1974. Bulbodacnitis alpinus sp. nov. (Nematoda: Cucullanidae) from Arctic char, Salvelinus alpinus L., with notes on other species of Bulbodacnitis. Canadian Journal of Zoology. 52: 411-446.

Munroe, E. G. 1949. Notes on the fish of the interior of the Labrador peninsula. Arctic. 2: 105-173.

Neave, F. and Bajkov, A. 1929. Reports of the Jasper Park lakes investigations, 1925-1926. V. Food and growth of Jasper Park fishes. Contributions to Canadian Biology \& Fisheries, New Series. 4: 197-219.

Nelson, J. S. 1965. Effects of fish introductions and hydroelectric development on fishes in the Kananaskis River system, Alberta. Journal of the Fisheries Research Board of Canada. 22: 721-753.

Nelson, J. S. and Paetz, M. J. 1992. The Fishes of Alberta. University of Alberta \& University of Calgary Press, Edmonton \& Calgary, Alberta. 437 p.

Nelson, P., Choudhury, A. and Dick, T. A. 1997. Crepidostomum percopsi n. sp. (Digenea: Allocreadiidae) from the trout perch (Percopsis omiscomaycus) of Dauphin Lake, Canada. Journal of Parasitology. 83: 1157-
1160.

Nener, J., Kieser, D., Thompson, J. A. , Lockhart, W. L., Metner, D. A. and Roome, R. 1995. Monitoring of mountain whitefish Prosopium williamsoni, from the Columbia River system near Castlegar, British Columbia: health parameters and contaminants in 1992. Canadian Technical Report of Fisheries \& Aquatic Sciences. No. 2036: xi +89 p.

Newton, M. V. B. 1932. The biology of Triaenophorus tricuspidatus (Bloch, 1779) in western Canada. Contributions to Canadian Biology \& Fisheries, New Series. 7: 341-360.

Nicholson, D. 1928. Fish tapeworm. Intestinal infection in man: the infestation of fish in Manitoba lakes. Canadian Medical Association Journal. 19: 25-33.

Nicholson, D. 1929. Variation in the fish larvae of Diphyllobothrium latum. Canadian Journal of Public Health. 20: 193-195.

Nicholson, D. 1932a. The Triaenophorus parasite in the flesh of the tullibee (Leucichthys). Canadian Journal of Research, Section D. 6: 162-165.

Nicholson, D. 1932b. Diphyllobothrium infection in Esox lucius. Canadian Journal of Research, Section D. 6: 166-170.

Oakland, G. B. 1948. Appendix No. 14. Statistical studies of whitefish infestation, $p$. 23-24. In: K. H. Doan. Fisheries Research Board of Canada. Annual Report of the Central Fisheries Research Station for 1948, with Investigators' Summaries as Appendices.

Oakland, G. B. 1949. Appendix No. 9. 
Statistical studies of whitefish infestation, $p$. 30-31. In: K. H. Doan. Fisheries Research Board of Canada. Annual Report of the Central Fisheries Research Station for 1949, with Investigators' Summaries as Appendices.

Oakland, G. B. 1950. Infestation rate studies Triaenophorus crassus. Fisheries Research Board of Canada. Manuscript Report (Biology). No. 486: 30 p.

Oliver, D. R. 1960. The macroscopic bottom fauna of Lac la Ronge, Saskatchewan. Journal of the Fisheries Research Board of Canada. 17: 607-624.

Paetz, M. J. and Nelson, J. S. 1970. The Fishes of Alberta. Government of Alberta, Queen's Printer, Edmonton, Alberta. 281 p.

Parnell, I. W. 1934. Fish parasites and their importance. Transactions of the American Fisheries Society. 64: 390-400.

Petrochenko, V. I. 1956. Acanthocephala of Domestic and Wild Animals. Volume I. Izdatel'stvo Akademiya Nauk SSSR. Moscow. 465 p. [Translation: Israel Program for Scientific Translations. Jerusalem, No. 5901. 1971].

Pike, A. W. and Burt, M. D. B. 1981. Host tissue response of the yellow perch (Perca flavescens) to three species of encysted metacercariae (Platyhelminthes: Digenea). Program \& Abstracts. 20 $0^{\text {th }}$ Annual Meeting, Canadian Society of Zoologists. Bulletin of the Canadian Society of Zoologists. 12(2): 76.

Pike, A. W. and Burt, M. D. B. 1982. Tissue response of Percaflavescens (yellow perch) to infection with Apophallus brevis
(Heterophyidae) (the sandgrain worm). Parasitology. 84(1): xlviii.

Pike, A. W. , Thompson, A. B. and Burt, M. D. B. 1983. The distribution of Apophallus brevis metacercariae in the yellow perch Perca flavescens. Parasitology. 87(2): xxix.

Pippy, J. H. C. and Sandeman, I. M. 1967. A kill of brook trout (Salvelinus fontinalis) involving the acanthocephalan Echinorhynchus lateralis. Journal of the Fisheries Research Board of Canada. 24: 1627-1628.

Poole, B. C. 1983. Fish-parasite population dynamics in small boreal lakes of central Canada. M. Sc. Thesis. Department of Zoology, University of Manitoba, Winnipeg, Manitoba.

Poole, B. C. and Dick, T. A. 1983. Metacercarial distribution of Apophallus brevis (Heterophyidae) in yellow perch (Perca flavescens) from Heming Lake study area. Canadian Journal of Zoology. 61: 2104-2109.

Poole, B. C. and Dick, T. A. 1984. Liver pathology of yellow perch, Perca flavescens (Mitchill), infected with larvae of the nematode Raphidascaris acus (Bloch, 1779). Journal of Wildlife Diseases. 20: 303-307.

Poole, B. C. and Dick, T. A. 1985. Parasite recruitment by stocked walleye, Stizostedion vitreum vitreum (Mitchill), fry in a small boreal lake in central Canada. Journal of Wildlife Diseases. 21: 371-376.

Poole, B. C. and Dick, T. A. 1986. Raphidascaris acus (Bloch, 1779) in northern pike, Esox lucius L., walleye, Stizostedion vitreum vitreum (Mitchill), and yellow perch, 
Perca flavescens (Mitchill), from central Canada. Journal of Wildlife Diseases. 22: 435436.

Poulin, R., Conley, D. C. and Curtis, M. A 1990. Effects of temperature fluctuations and photoperiod on hatching in the parasitic copepod Salmincola edwardsii. Canadian Journal of Zoology. 68: 1330-1332.

Poulin, R., Curtis, M. A. and Rau, M. E. 1989. Responses of the parasitic copepod Salmincola edwardsii to visual and mechanical stimuli and their role in host location. Program \& Abstracts. $28^{\text {th }}$ Annual Meeting, Canadian Society of Zoologists. Bulletin of the Canadian Society of Zoologists. 20(2): 53.

Poulin, R., Curtis, M. A. and Rau, M. E 1990. Responses of the fish ectoparasite Salmincola edwardsii (Copepoda) to stimulation, and their implication for host finding. Parasitology. 100: 417-421.

Poulin, R., Curtis, M. A. and Rau, M. E. 1991. Size, behaviour, and acquisition of ectoparasitic copepods by brook trout, Salvelinus fontinalis. Oikos. 61: 169-174.

Poulin, R., Curtis, M. A. and Rau, M. E. 1992. Effects of Eubothrium salvelini (Cestoda) on the behaviour of Cyclops vernalis (Copepoda) and its susceptibility to fish predators. Parasitology. 105: 265-271.

Poulin, R. and Fitzgerald, G. J. 1987. The potential of parasitism in the structuring of a salt marsh stickleback community. Canadian Journal of Zoology. 65: 2793-2798.

Poulin, R. and Fitzgerald, G. J. 1988. Water temperature, vertical distribution, and risk of ectoparasitism in juvenile sticklebacks. Canadian Journal of Zoology. 66: 2002-2005.

Poulin, R. and Fitzgerald, G. J. 1989a. A possible explanation for the aggregated distribution of Argulus canadensis Wilson, 1916 (Crustacea: Branchiura) on juvenile sticklebacks (Gasterosteidae). Journal of Parasitology. 75: 58-60.

Poulin, R. and Fitzgerald, G. J. 1989b. Risk of parasitism and microhabitat selection in juvenile sticklebacks. Canadian Journal of Zoology. 67: 14-18.

Poulin, R. and Fitzgerald, G. J. 1989c. Malebiased sex ratio in Argulus canadensis Wilson, 1916 (Crustacea: Branchiura) ectoparasitic on sticklebacks. Canadian Journal of Zoology. 67 : 2078-2080.

Poulin, R. and Fitzgerald, G. J. 1989d. Shoaling as an anti-parasitic mechanism in juvenile sticklebacks (Gasterosteus spp.). Behavioural Ecology and Sociobiology. 24: 251-255.

Price, C. E. and Arai, H. P. 1967. The monogenean parasites of Canadian freshwater fishes. Canadian Journal of Zoology. 45: 12351245.

Price, E. W. 1942. North American monogenetic trematodes. V. The family Hexabothriidae, n. n. (Polystomatoidea). Proceedings of the Helminthological Society of Washington. 9: 39-56.

Pritchard, A. L. 1931. Taxonomic and life history studies of the ciscoes of Lake Ontario. Ontario Fisheries Research Laboratory Publication. No. 41: 78 p. (University of 
Toronto Studies in Biology. 35: 1-78).

Prychitko, S. B. and Nero, R. W. 1983. Occurrence of the acanthocephalan Echinorhynchus leidyi (Van Cleave, 1924) in Mysis relicta. Canadian Journal of Zoology. 61: 460-462.

Pybus, M. J. and Samuel, W. M. 1978. Summary of field trips - Wildlife Parasitology Class. Unpublished Report. Department of Zoology, University of Alberta, Edmonton, Alberta. $14 \mathrm{p}$.

Raikova, W. V. 1994. Life cycle, cytology, and morphology of Polypodium hydriforme, a coelenterate parasite of the eggs of acipenseriform fishes. Journal of Parasitology. 80: $1-12$.

Rau, M. E. and Gordon, D. M. 1977. A technique for the demonstration of the metacercariae of Apatemon gracilis pullucidus (Yamaguti, 1933) in the deep tissues of the brook stickleback (Culaea inconstans). Canadian Journal of Zoology. 55: 1200-1201.

Rau, M. E. and Gordon, D. M. 1978. The frequency distribution of tetracotyles of Apatemon gracilis pellucidus (Yamaguti, 1933) in stickleback Culaea inconstans (Kirtland) populations of homogeneous age and size. Journal of Fish Diseases. 1: 259-263.

Rau, M. E., Gordon, D. M. and Curtis, M. A. 1979. Bilateral symmetry of Diplostomum infections in the eyes of lake whitefish Coregonus clupeaformis (Mitchill) and a computer simulation of the observed metacercarial distribution. Journal of Fish Diseases. 2: 291-297.
Rawson, D. S. 1930. The bottom fauna of Lake Simcoe and its role in the ecology of the lake. University of Toronto Studies. Publications of the Ontario Fisheries Research Laboratory, No. 40: 183 p.

Rawson, D. S. A biological survey and recommendations for fisheries management in waters of Banff National Park. Canada Department of Mines \& Resources. National Parks Bureau, National \& Historic Parks Branch. 177 p.

Rawson, D. S. 1945. The failure of rainbow trout and initial success with the introduction of lake trout in Clear Lake, Riding Mountain Park, Manitoba. Transactions of the American Fisheries Society. 75: 323-335.

Rawson, D. S. 1946a. Appendix VI. Reports for 1945 on the Northwestern Fisheries Investigations. B. Great Slave Lake Investigation, p. 43. In: A. T. Cameron. Annual Report of the Fisheries Research Board of Canada for the year 1945.

Rawson, D. S. 1946b. Appendix VI. Reports for 1945 on the Northwestern Fisheries Investigations. A. Lake Athabasca Investigation, p. 42. In: A. T. Cameron. Annual Report of the Fisheries Research Board of Canada for the year 1945.

Rawson, D. S. 1947a. Chapter V. Great Slave Lake, p. 45-68. In: Northwest Canadian Fisheries Surveys in 1944-1945. Fisheries Research Board of Canada. Bulletin No. 72.

Rawson, D. S. 1947b. Chapter VI. Lake Athabasca, p. 69-85. In: Northwest Canadian Fisheries Surveys in 1944-1945. Fisheries Research Board of Canada. Bulletin No. 72. 
Rawson, D. S. 1947c. Deterioration of recently established trout populations in lakes of the Canadian Rockies. Canadian Fish Culturist. 3: 14-21.

Rawson, D. S. 1951. Studies of the fish of Great Slave Lake. Journal of the Fisheries Research Board of Canada. 8: 207-240.

Rawson, D. S. 1953. The limnology of Amethyst Lake, a high alpine type near Jasper, Alberta. Canadian Journal of Zoology. 31: 193-210.

Rawsor, D. S. 1957a. The life history and ecology of the yellow walleye, Stizostedion vitreum in Lac la Ronge, Saskatchewan. Transactions of the American Fisheries Society. $86:$ 15-37.

Rawson, D. S. 1957b. Limnology and fisheries of five lakes in the Upper Churchill Drainage, Saskatchewan. Saskatchewan Department of Natural Resources. Fisheries Report No. 3. 61 p.

Rawson, D. S. 1959. Limnology and fisheries of Cree and Wollaston lakes in northern Saskatchewan. Saskatchewan Department of Natural Resources. Fisheries Branch Report. No. 4: 73 p.

Rawson, D. S. 1960. Limnology and fisheries of five lakes on the Churchill River near Stanley, Saskatchewan. Saskatchewan Department of Natural Resources. Fisheries Branch, Fisheries Report. 5: 38 p.

Rawson, D. S. 1961. The lake trout of Lac la Ronge, Saskatchewan. Journal of the Fisheries Research Board of Canada. 18: 423-462.
Rawson, D. S. and Elsey, C. A. 1950. The reduction of the longnose sucker population of Pyramid Lake, Alberta, in an attempt to improve angling. Transactions of the American Fisheries Society. 78: 13-31.

Rawson, D. S. and Wheaton, R. R. 1950. Appendix No. 4. Studies of Triaenophorus crassus in Nesslin Lake, Saskatchewan, p. 1820. In: K. H. Doan. Fisheries Research Board of Canada. Annual Report of the Central Fisheries Research Station for 1950, with Investigators' Summaries as Appendices.

Reed, E. B. 1962. Limnology and fisheries of the Saskatchewan River in Saskatchewan. Saskatchewan Department of Natural Resources. Fisheries Branch, Fisheries Report. No. 6: $48 \mathrm{p}$.

Reed, G. B. and Dymond, J. R. 1949. Appendix VII. Report for 1948 of the Central Fisheries Research Station, Winnipeg, Man., 64-66. In: G. B. Reed and J. R. Dymond. Annual Report of the Fisheries Research Board of Canada for the year 1948.

Reed, G. B. and Dymond, J. R. 1951. Central Fisheries Research Station, Winnipeg, Man., p. 52-61. In: G. B. Reed and J. R. Dymond. Annual Report of the Fisheries Research Board of Canada for the year 1950.

Reed, G. B. and Dymond, J. R. 1952. Central Fisheries Research Station, Winnipeg, Manitoba, p. 37-42. In: G. B. Reed and J. R. Dymond. Annual Report of the Fisheries Research Board of Canada for the year 1951.

Reed, G. B. and Dymond, J. R. 1953. Central Fisheries Research Station, Winnipeg, Man. P. 71-81. In: G. B. Reed and J. R. Dymond. 
Annual Report of the Fisheries Research Board of Canada for the year 1952.

Reimchen, T. E. 1982. Incidence and intensity of Cyathocephalus truncatus and Schistocephalus solidus infections in Gasterosteus aculeatus. Canadian Journal of Zoology. 60: 1091-1095.

Reist, J. D., Bodaly, R. A., Fudge, R. J. P., Cash, K. J. and Stevens, T. V. 1987. External scarring of whitefish, Coregonus nasus and Coregonus clupeaformis complex, from the western Northwest Territories, Canada. Canadian Journal of Zoology. 65: 1230-1239.

Richardson, L. R. 1935. A record of Octomitus salmonis Moore from Quebec. Transactions of the American Fisheries Society. 65: 290-292.

Richardson, L. R. 1936. Observations on the parasites of the speckled trout in Lake Edward, Quebec. Transactions of the American Fisheries Society. 66: 343-356.

Richardson, L. R. 1937. Raphidascaris laurentianus sp. n. (Ascaroidea) from Salvelinus fontinalis (Mitchill) in Quebec. Canadian Journal of Research, Section D. 15: 112-115.

Richardson, L. R. 1938. An account of a parasitic copepod, Salmincola salvelini sp. nov., infecting the speckled trout. Canadian Journal of Research, Section D. 16: 225-229.

Richardson, L. R. 1942. The parasites of fishes of Lake Wakonichi, central northern Quebec. Transactions of the American Fisheries Society. 71: 286-289.
Ricker, W. E. 1932. Studies of speckled trout (Salvelinus fontinalis) in Ontario. University of Toronto Studies. Publications of the Ontario Fisheries Research Laboratory. No. 44: $41 \mathrm{p}$.

Roberts, L. S. 1963. Ergasilus nerkae n. sp. (Copepoda: Cyclopoida) from British Columbia with discussion of the copepods of the $E$. caeruleus group. Canadian Journal of Zoology. 41: 115-124.

Roberts, L. S. 1970. Ergasilus (Copepoda: Cyclopoida): revision and key to the species in North America. Transactions of the American Microscopical Society. 89: 134-161.

Rosen, R. and Dick, T. A. 1982. The biology of the plerocercoid of Triaenophorus crassus Forel in experimentally infected whitefish Coregonus clupeaformis (Mitchill). Abstracts. $5^{\text {th }}$ International Congress of Parasitology. Molecular and Biochemical Parasitology, Supplement. p. 384.

Rosen, R. and Dick, T. A. 1983. Development and infectivity of the procercoid of Triaenophorus crassus Forel and mortality of the first intermediate host. Canadian Journal of Zoology. 61: 2120-2128.

Rosen, R. and Dick, T. A. 1984a. Growth and migration of plerocercoids of Triaenophorus crassus Forel and pathology in experimentally infected whitefish, Coregonus clupeaformis (Mitchill). Canadian Journal of Zoology. 62: 203-211.

Rosen, R. and Dick, T. A. $1984 \mathrm{~b}$. Experimental infections of rainbow trout, Salmo gairdneri Richardson, with plerocercoids of Triaenophorus crassus Forel. 
Journal of Wildlife Diseases. 20: 34-38.

Rosen, R. and Dick, T. A. 1984c. Experimental infections of rainbow trout, Salmo gairdneri Richardson, with plerocercoids of Triaenophorus crassus Forel. Program \& Abstracts. 59 $9^{\text {th }}$ Annual Meeting, American Society of Parasitologists. p. 46.

Roussow, G. 1954. La ligulose des catostomes du Lac Lauzon. Annals de l'Association Canadienne-Française pour l'Avancement des Sciences. 20: 85-90.

Roussow, G. 1960. Quelques parasites de poisson qui peut etre dangereux pour l'homme. Ministère de la Chasse et Pécheries, Québec, Office de Biologie. Journal de Bord. 3: 398-401.

Russell, L. R. 1980. Effects of Truttaedacnitis truttae (Nematoda: Cucullanidae) on the growth and swimming of rainbow trout, Salmo gairdneri. Canadian Journal of Zoology. 58: 1220-1226.

Ryder, J. P. 1963. Parasites of walleyes from two central Alberta lakes. Unpublished Report. Department of Zoology, University of Alberta, Edmonton, Alberta. 16 p.

Ryerson, C. G. S. 1915. Notes on the Hirudinea of Georgian Bay. Contributions to Canadian Biology. 1911-1914: 165-175.

Samuel, W. M. 1981. Parasites of fishes of William A. Switzer Provincial Park, 19751981. Manuscript Report. Department of Zoology, University of Alberta, Edmonton, Alberta. 9 p.

Samuel, W. M. 1985. Parasites of fish from
William A. Switzer Provincial Park - 19751985 with specific emphasis on results of the February 1985 collection. Unpublished Report. Department of Zoology, University of Alberta, Edmonton, Alberta. 12 p.

Sandeman, I. M. and Pippy, J. H. C. 1967. Parasites of freshwater fishes (Salmonidae and Coregonidae) of insular Newfoundland. Journal of the Fisheries Research Board of Canada. 24: 1911-1943.

Savage, J. 1935. Copepod infections of speckled trout. Transactions of the American Fisheries Society. 65: 334-339.

Scott, W. B. and Crossman, E. J. 1973. Freshwater Fishes of Canada. Bulletin of the Fisheries Research Board of Canada. 184: 966 p.

Shepherd, C. D. and Mace, T. F. 1980. Octomacrum microconfibula (Monogenea: Polyopisthocotylea) from a British Columbia lake. Journal of Parasitology. 66: 913

Shostak, A. W. and Dick, T. A. 1982. Appearance of a new morph of Echinorhynchus Müeller 1776 following watershed diversion. Abstracts. $5^{\text {th }}$ International Congress of Parasitology. Molecular and Biochemical Parasitclogy. Supplement. p. 609-610.

Shostak, A. W. and Dick, T. A. 1986. Intestinal pathology in northern pike, Esox lucius $\mathrm{L}$., infected with Triaenophorus crassus Forel, 1868 (Cestoda: Pseudophyllidea) Journal of Fish Diseases. 9: 35-45.

Shostak, A. W. and Dick, T. A. 1987a. Seasonal changes in site occupation by 
intestinal helminths of northern pike provides evidence for space-mediated inter-specific interaction. Program \& Abstracts. $62^{\text {nd }}$ Annual Meeting, American Society of Parasitologists. p. 28.

Shostak, A. W. and Dick, T. A. 19876. Individual variability in reproductive success of the cestode Triaenophorus crassus Forel. Program \& Abstracts. 26 $6^{\text {th }}$ Annual Meeting, Canadian Society of Zoologists. Bulletin of the Canadian Society of Zoologists. 18(2): 36.

Shostak, A. W. and Dick, T. A. 1987c. Individual variability in reproductive success of the cestode Triaenophorus crassus Forel (Cestoda: Pseudophyllidea), with comments one the use of the Lorenz curve and Gini coefficient. Canadian Journal of Zoology. 65: 2878-2885.

Shostak, A. W. and Dick, T. A. 1989a. Variability in timing of egg hatch of Triaenophorus crassus Forel (Cestoda: Pseudophyllidea) as a mechanism increasing temporal dispersion of coracidia. Canadian Journal of Zoology. 67: 1462-1470.

Shostak, A. W. and Dick, T. A. $1989 \mathrm{~b}$. Helminth position within the intestine of naturally infected pike (Esox lucius) relative to host stomach contents. Journal of Parasitology. 75: 905-910.

Shostak, A. W. , Dick, T. A., Szalai, A. J. and Bernier, L. M. J. 1986. Morphological variability in Echinorhynchus gadi, E. leidyi, and E. salmonis (Acanthocephala: Echinorhynchidae) from fishes in northern Canadian waters. Canadian Journal of Zoology. 64: 985-995.
Shostak, A. W. , Rosen, R. B. and Dick, T. A. 1984. Orientation of procercoids of Triaenophorus crassus Forel in Cyclops bicuspidatus thomasi Forbes: effects on growth and development. Canadian Journal of Zoology. 62: 1373-1377.

Shostak, A. W., Rosen, R. B. and Dick, T. A. 1985. The use of growth curves to assess the crowding effect on procercoids of the tapeworm Triaenophorus crassus in the copepod host Cyclops bicuspidatus thomasi. Canadian Journal of Zoology. 63: 2343-2351.

Shostak, A. W., Thompkins, J. C. and Dick, T. A. 1987. The identification and morphological variation of Diplostomum baeri bucculentum from two gull species, using metacercarial infections from the least cisco from the Northwest Territories. Canadian Journal of Zoology. 65: 2287-2291.

Sinclair, N. R. 1972a. Studies on the heterophyid trematode Apophallus brevis, the "sandgrain grub" of yellow perch (Perca flavescens). I. Redescription and resolution of synonymic conflict with Apophallus imperator Lyster, 1940 and other designations. Canadian Journal of Zoology. 50: 357-364.

Sinclair, N. R. 1972b. Studies on the heterophyid trematode, Apophallus brevis, the "sand-grain grub" of yellow perch (Perca flavescens). II. The metacercaria: position, structure, and composition of the cyst; hosts, geographical distribution and variation. Canadian Journal of Zoology. 50: 577-584.

Skinker, M. S. 1930. ["Note on a new species of nematode of the genus Cystidicola from fishes.]. Proceedings of the Helminthological Society of Washington, $121^{\text {st }}$ Meeting. Journal 
of Parasitology. 16: 167. *(a title was not cited for this note)

Skinker, M. S. 1931. Three new parasitic worms. Proceedings of the United States National Museum (2890). 79(Article 24): 1-9.

Smedley, E. M. 1933. Nematode parasites from Canadian marine and freshwater fishes. Contributions to Canadian Biology and Fisheries, New Series. 8: 169-179.

Smedley, E. M. 1934. Some parasitic nematodes from Canadian fishes. Journal of Helminthology. 12: 205-220.

Smith, J. D. 1984a. Taxonomy of Raphidascaris spp. (Nematoda, Anisakidae) of fishes, with a redescription of $R$. acus (Bloch, 1772). Canadian Journal of Zoology. 62: 685694.

Smith, J. D. 1984b. Development of Raphidascaris acus (Nematoda: Anisakidae) in paratenic, intermediate and definitive hosts. Canadian Journal of Zoology. 62: 1378-1386.

Smith, J. D. Seasonal transmission of Raphidascaris acus (Nematoda: Anisakidae), a parasite of freshwater fishes, in definitive and intermediate hosts. Environmental Biology of Fishes. 16: 295-308.

Smith, J. D. and Anderson, R. C. 1981. Seasonality of Raphidascaris canadensis (Nematoda: Anisakidae) in the intermediate host, yellow perch (Perca flavescens). Program \& Abstracts. $20^{\text {th }}$ Annual Meeting, Canadian Society of Zoologists. Bulletin of the Canadian Society of Zoologists. 12(2): 86.

Smith, J. D. and Anderson, R. C. 1982.
Seasonality of Raphidascaris acus (Nematoda) in intermediate and definitive hosts. Abstracts. $5^{\text {th }}$ International Congress of Parasitology. Molecular and Biochemical Parasitology. Supplement. p. 352-353.

Smith, J. D. and Lankester, M. W. 1979. Development of the swim bladder nematodes (Cystidicola spp.) in their intermediate hosts. Canadian Journal of Zoology. 57: 1736-1744.

Smith, J. D. and Kramer, D. L. 1987. Effects of the cestode (Schistocephalus sp.) on the response of ninespine sticklebacks (Pungitius pungitius) to aquatic hypoxia. Canadian Journal of Zoology. 65: 1862-1865.

Stafford, J. 1902. Notes on worms. Zoologischer Anzeiger. 25: 481-483.

Stafford, J. 1904. Trematodes from Canadian fishes. Zoologischer Anzeiger. 27: 481-495.

Steedman, R. J. 1991. Occurrence and environmental correlates of black spot disease in stream fishes near Toronta, Ontario. Transactions of the American Fisheries Society. 120: 494-499.

Stewart, D. B. and Bernier, L. M. J. 1982. An aquatic survey of islands bordering Viscourt Melville Sound, District of Franklin, Northwest Territories. Environment Canada \& Department of Indian \& Northern Affairs, Northern Environmental Protection Branch, Lands Directorate. Northern Land Use Series, Background Report. No. 2: 110 p.

Stewart, D. B. and Bernier, L. M .J. 1983. An aquatic resource survey of King William and Victoria islands, and the northeastern District of Keewatin, Northwest Territories. 
Environment Canada \& Department of Indian and Northern Affairs, Northern Environmental Protection Branch, Lands Directorate, Northern Land Use Series, Background Report. No. 3: 127 p.

Stewart, D. B. and Bernier, L. M. J. 1984. An aquatic resource survey of Melville Peninsula, Southhampton Island, and the northeastern District of Keewatin, Northwest Territories. Environment Canada \& Department of Indian \& Northern Affairs, Northern Environment Directorate. Background Report. No. 4: 144 p.

Stewart-Hay, R. K. 1951a. A report on the fishes of Childs Lake - Summer 1951. Manitoba Department of Mines \& Natural Resources. Game \& Fisheries Branch Report. $4 \mathrm{p}$.

Stewart-Hay, R. K. 1951b. A biological survey of Lake Daupin - Summer 1951. Manitoba Department of Mines \& Natural Resources. Game \& Fisheries Branch Report, $44 \mathrm{p}$.

Stewart-Hay, R. K. 1951c. A report on the fishes of Glad Lake - Summer 1951. Manitoba Department of Mines \& Natural Resources. Game \& Fisheries Branch Report. 4 p.

Stewart-Hay, R. K. 1951d. A report on the fishes of Laurie Lake - Summer 1951. Manitoba Department of Mines \& Natural Resources. Game \& Fisheries Branch Report. $2 \mathrm{p}$.

Stewart-Hay, R. K. 1951e. A report on the fishes of Singoosh Lake - July 1951. Manitoba Department of Mines \& Natural Resources. Game \& Fisheries Branch Report. 5 p.
Stewart-Hay, R. K. 1951f. A biological survey of Snow Lake, Manitoba. Manitoba Department of Mines \& Natural Resources. Game \& Fisheries Branch Report. 20 p.

Stewart-Hay, R. K. 1951g. A biological survey of Wellman Lake - Summer 1951. Manitoba Department of Mines \& Natural Resources. Game \& Fisheries Branch Report. 20 p.

Stewart-Hay, R. K. 1951h. A report on the fishes of West Blue Lake - Summer 1951. Manitoba Department of Mines \& Natural Resources. Game \& Fisheries Branch Report. $4 \mathrm{p}$.

Stewart-Hay, R. K. 1952a. A limited biological survey of Burton Lake - August 1952. Manitoba Department of Mines \& Natural Resources. Game \& Fisheries Branch Report. 8 p.

Stewart-Hay, R. K. 1952b. An investigation of certain waters in the Porcupine Mountains Summer 1952. Manitoba Department of Mines \& Natural Resources. Game \& Fisheries Branch Report. 114 p.

Stewart-Hay, R. K. 1953a. A biological survey of Lake Athapapaskow - July 1953. Manitoba Department of Mines \& Natural Resources. Game \& Fisheries Branch Report. 79 p.

Stewart-Hay, R. K. 1953b. A brief biological survey of Second Cranberry Lake - August 1953. Manitoba Department of Mines \& Natural Resources. Game Fisheries Branch Report. 19 p.

Stewart-Hay, R. K. 1953c. A biological survey of three unnamed lakes in the Whiteshell Forest Reserve - August 1953. Manitoba 
Department of Mines \& Natural Resources. Game \& Fisheries Branch Report. 17 p.

Stock, T. M. 1988. Helminth parasites of lake whitefish, Coregonus clupeaformis, and northern pike, Esox lucius, from Karvis Lake, Alberta. Unpublished Report. Department of Zoology, Unversity of Alberta, Edmonton, Alberta. 12 p.

Sunde, L. A. 1963a. Southern Indian Lake whitefish infestation test - March $5^{\text {th }}$ to $7^{\text {th }}$, 1963. Manitoba Department of Mines \& Natural Resources. Fisheries Branch Report. 3 p.

Sunde, L. A. 1963b. Summary of whitefish infestation tests conducted in northern Manitoba in 1963. Manitoba Department of Mines \& Natural Resources. Fisheries Branch Report. 2 p.

Sunde, L. A. 1964. Summary of whitefish infestation tests conducted in northern Manitoba in 1964. Manitoba Department of Mines \& Natural Resources. Fisheries Branch Report. 3 p.

Sunde, L. A. 1965. Summary of Department of Fisheries whitefish infestation tests for northern Manitoba (August 1962 to February 1965). Manitoba Department of Mines \& Natural Resources. Fisheries Branch Report. $4 \mathrm{p}$.

Swanson, G. M., Kansas, K. R., Matkowski, S. M. \& Graveline, P. 1991. A report on the fisheries resources of the lower Nelson River and the impact of hydroelectric development, 1989 data. Manitoba Department of Natural Resources. Fisheries Branch, Manuscript Report. 91-03: 248 p.
Szalai, A. J. 1989. Factors affecting community structure, transmission and regulation of fish-parasites in Dauphin Lake, Manitoba. Ph. D. Dissertation. Department of Zoology, University of Manitoba, Winnipeg, Manitoba. 309 p.

Szalai, A. J, Craig, J. F. and Dick, T. A. 1992. Parasites of fishes from Dauphin Lake, Manitoba, 1985-1987. Canadian Technical Report of Fisheries \& Aquatic Sciences. No. 1735: iv + $36 \mathrm{p}$.

Szalai, A. J. and Dick, T. A. 1987a. Parasites of quillback, Carpiodes cyprinus (Lesueur) from Dauphin Lake, Manitoba, Canada. Journal of Parasitology. 73: 446-447.

Szalai, A. J. and Dick, T. A. 1987b. Intestinal pathology and site specificity of the acanthocephalan Neoechinorhynchus carpiodi Dechtiar, 1968, in quillback, Carpiodes cyprinus (Lesueur). Journal of Parasitology. 73: 467-475.

Szalai, A. J. and Dick, T. A. 1988. Helminths of stocked rainbow trout (Salmo gairdneri) with special reference to Clinostomum complanatum. Journal of Wildlife Diseases. 24: $456-460$.

Szalai, A. J. and Dick, T. A. 1989. Differences in numbers and inequalities in mass and fecundity during the egg-producing period for Raphidascaris acus (Nematoda: Anisakidae). Parasitology. 98: 489-495.

Szalai, A. J. and Dick, T. A. 1991a. Role of predation and parasitism in growth and mortality of yellow perch in Dauphin Lake, Manitoba. Transactions of the American Fisheries Society. 120: 739-751. 
Szalai, A. J. and Dick, T. A. $1991 \mathrm{~b}$. Evaluation of gill nets, fyke nets, and markrecapture methods to estimate the numbers of Hirudinea and Crustacea on fish. Journal of Parasitology. 77: 914-922.

Szalai, A. J., Lysack, W. and Dick, T. A. 1992. Use of confidence ellipses to detect effects of parasites on the growth of yellow perch, Perca flavescens. Journal of Parasitology. 78: 64-69.

Szalai, A. J., Yang, X. and Dick, T. A. 1989. Changes in numbers and growth of Ligula intestinalis in the spottail shiner (Notropis hudsonius), and their roles in transmission. Journal of Parasitology. 75: 571-576.

Taylor, L. H., Hall, B. K. and Cone, D. K. 1993. Experimental infection of yellow perch (Perca flavescens) with Apophallus brevis (Digenea: Heterophyidae): parasite invasion, encystment and bony ossicle development. Canadian Journal of Zoology. 71: 1886-1894.

Tedla, S. 1969. The ecology of some metazoan parasites of the yellow perch, Perca fluviatilis L. Ph. D. Dissertation. Department of Biology, University of Waterloo, Waterloo, Ontario. 208 p.

Tedla, S. and Fernando, C. H. 1969a. Observations on the seasonal changes of the parasite fauna of yellow perch (Perca flavescens) from the Bay of Quinte, Lake Ontario. Journal of the Fisheries Research Board of Canada. 26: 883-843.

Tedla, S. and Fernando, C. H. $1969 \mathrm{~b}$. Observations on the biology of Ergasilus spp. (Cyclopoidea: Copepoda) infesting North American freshwater fishes. Canadian Journal of Zoology. 47: 405-408.

Tedla, S. and Fernando, C. H. 1969c. Occurrence of plerocercoids of Triaenophorus nodulosus (Pallas, 1781) in the white perch Roccus americanus (Gmelin). Journal of Parasitology. 55: 334.

Tedla, S. and Fernando, C. H. 1969d. Observations of the glochidia of Lampsilis radiata (Gmelin) infesting yellow perch, Perca flavescens (Mitchill) in the Bay of Quinte, Lake Ontario. Canadian Journal of Zoology. 47: 705-712.

Tedla, , S. and Fernando, C. H. 1970a. Some aspects of the ecology of the parasite fauna of the gills of yellow perch, Perca flavescens. Journal of the Fisheries Research Board of Canada. 27: 1045-1050.

Tedla, S. and Fernando, C. H. 1970b. Some remarks on the ecology of Echinorhynchus salmonis Müller, 1784. Canadian Journal of Zoology. 48: 317-321.

Tedla, S. and Fernando, C. H. 1970c. On the biology of Ergasilus confusus Bere, 1931 (Copepoda), infesting yellow perch, Perca fluviatilis L., in the Bay of Quinte, Lake Ontario, Canada. Crustaceana. 19: 1-14.

Tedla, S. and Fernando, C. H. 1972. On the characterization of the parasite fauna of yellow perch (Perca fluviatilis) in five lakes in southern Ontario. Helminthologia (Bratislavia). 11: 23-33.

Thomas, L. J. 1946. New pseudophyllidean cestodes from the Great Lakes region. I. Diphyllobothrium oblongatum n. sp. from gulls. Journal of Parasitology. 32: 1-6. 
Thompson, G. E. 1974. The ecology and life history of the mountain whitefish (Prosopium williamsoni Girard) in the Sheep River, Alberta. M. Sc. Thesis. Department of Biology, University of Calgary, Calgary, Alberta.

Thompson, P. A. and Threlfall, W. 1978. The metazoan parasites of two species of fish from the Port-Cartier-Sept-Iles Park, Quebec. Le Naturaliste Canadien. 205: 429-431.

Threlfall, W. 1968. A mass die-off of the three-spined stickleback (Gasterosteus aculeatus L.) caused by parasites. Canadian Journal of Zoology. 46: 105-106.

Threlfall, W. 1974. New and previously described species of monogenetic trematodes from Labrador catostomids and cyprinids. Folia Parasitologia (Praha). 21: 205-214.

Threlfall, W. 1986. Seasonal occurrence of Anodonta cataracta glochidia on three-spined sticklebacks, Gasterosteus aculeatus. Veliger. 29: 231-234.

Threlfall, W. and Hanek, G. 1969. Capillaria salvelini Polyanski, 1952 from Salvelinus fontinalis (Mitchill). Canadian Journal of Zoology. 47: 1088-1090.

Threlfall, W. and Hanek, G. 1970a. Metazoan parasites, excluding Monogenea, from longnose and white suckers. Journal of the Fisheries Research Board of Canada. 27: 1317-1319.

Threlfall, W. and Hanek, G. 1970b. Helminths from northern pike (Esox lucius L.) in Labrador. Journal of Parasitology. 56: 662.
Threlfall, W. and Hanek, G. 1970c. Metazoan parasites of salmonids and coregonids from the Avalon Peninsula, Newfoundland. Journal of the Fisheries Research Board of Canada. 27: 1894-1897.

Threlfall, W. and Hanek, G. 1971. Helminth parasites, excluding Monogenea, from some Labrador fishes. Journal of Parasitology. 57: 684-685.

Tidd, W. M. and Bangham, R. V. 1945. A new species of parasitic copepod, Ergasilus osburni, from the burbot. Transactions of the American Microscopical Society. 64: 225-227.

Uhazy, L. S. 1976. Philometroides huronensis n. sp. (Nematoda: Dracunculoidea) of the common white sucker (Catostomus commersoni) from Lake Huron. Canadian Journal of Zoology. 54: 369-376.

Uhazy, L. S. 1977a. Development of Philometroides huronensis (Nematoda: Dracunculoidea) in the intermediate and definitive hosts. Canadian Journal of Zoology. 55: 265-273.

Uhazy, L. S. 1977b. Biology of Philometroides huronensis (Nematoda: Dracunculoidea) in the white sucker (Catostomus commersoni). Canadian Journal of Zoology. 55: 1430-1441.

Uhazy, L. S. 1978. Lesions associated with Philometroides huronensis (Nematoda: Philometridae) in the white sucker (Catostomus commersoni). Journal of Wildlife Diseases. 14: 401-408.

Uhazy, L. S. and Anderson, R. C. 1976. Biology of Philometroides huronensis 
(Nematoda: Dracunculoidea) in the white sucker (Catostomus commersoni). Program \& Abstracts. $51^{\text {st }}$ Annual Meeting, American Society of Parasitologists. p.47.

Van Cleave, H. J. 1949. The acanthocephalan genus Neoechinorhynchus in the catostomid fishes in North America, with descriptions of two new species. Journal of Parasitology. 35 : 500-512.

Van Cleave, H. J. and Bangham, R. V. 1949. Four new species of the acanthocephalan family Neoechinorhynchidae from freshwater fishes of North America, one representing a new genus. Journal of the Washington Academy of Science. 39: 398-409.

Van Cleave, H. J. and Lynch, J. E. 1950. The circumpolar distribution of Neoechinorhynchusrutili, an acanthocephalan parasite of fishes. Transactions of the American Microscopical Society. 69: 156-171.

Vergeer, T. 1928a. Canadian fish, a source of the broad tapeworm of man in the United States. Journal to the American Medical Association. 90: 1687-1688.

Vergeer, T. 1928b. New sources of the broad tapeworm infestation: report of fourteenth native case. Journal ot the American Medical Association. 91: 396-397.

Vergeer, T. 1928c. An important source of broad tapeworm in America. Science, New Series. 68: 14-15.

Vergeer, T. 1928d. The dissemination of the broad tapeworm by wild Carnivora. Canadian Medical Association Journal. 19: 692-694.
Vergeer, T. 1929a. The dog, a reservoir of the broad tapeworm. Journal of the American Medical Association. 92: 607-608.

Vergeer, T. 1929b. The broad tapeworm in America, with suggestions for its control. Journal of Infectious Diseases. 44: 1-11.

Vergeer, T. 1942. Two new pseudophyllidean tapeworms of general distribution in the Great Lakes region. Transactions of the American Microscopical Society. 61: 373-382.

Wardle, R. A. 1932. The Cestoda of Canadian Fishes. II. The Hudson Bay drainage system. Contributions to Canadian Biology \& Fisheries, New Series. 7: 377-403.

Wardle, R. A. 1933a. The Cestoda of Canadian fishes. III. Additions to the Pacific coast fauna. Contributions to Canadian Biology \& Fisheries, New Series. 8: 77-78.

Wardle, R. A. 1933b. Significant factors in the plerocercoid environment of Diphyllobothrium latum (Linn.). Journal of Helminthology. 11: 25-44.

Wardle, R. A. 1933c. The parasitic helminths of Canadian animals. I. The Cestodaria and Cestoda. Canadian Journal of Research, Section D. 8: 317-333.

Wardle, R. A. 1935. Fish tape-worm. Biological Board of Canada. Bulletin No. 45: $25 \mathrm{p}$.

Watson, N. F. H. 1963a. A note on the upper lethal temperature of eggs of two species of Triaenophorus. Journal of the Fisheries Research Board of Canada. 20: 841-844. 
Watson, N. F. H. 1963b. Summer food of the lake whitefish, Coregonus clupeaformis Mitchill, from Heming Lake, Manitoba. Journal of the Fisheries Research Board of Canada. 20: 279-286.

Watson, N. F. H. and Lawler, G. H. 1956. Life history studies of Triaenophorus at Heming Lake, Manitoba. Part II. Incidence of Triaenophorus in the first intermediate host, Cyclops bicuspidatus. Fisheries Research Board of Canada. Manuscript Report (Biology). No. 622: 20 p.

Watson, N. F. H. and Lawler, G. H. 1961. Studies on the eggs and first intermediate stages of Triaenophorus at Heming Lake, Manitoba. Fisheries Research Board of Canada. Progress Report of the Biological Station and Technical Unit, London. No. 2: $51-52$.

Watson, N. F. H. and Lawler, G. H. 1963. Temperature and rate of hatching of Triaenophorus eggs. Journal of the Fisheries Research Board of Canada. 20: 249-251.

Watson, N. F. H. and Lawler, G. H. 1965. Natural infections of cyclopoid copepods with procercoids of Triaenophorus spp. at Heming Lake, Manitoba. Fisheries Research Board of Canada. Manuscript Report (Biology). No. 804: $35 \mathrm{p}$.

Watson, N. F. H. and Price, J. L. 1960. Experimental infections of cyclopoid copepods with Triaenophorus crassus Forel and $T$. nodulosus (Pallas). Canadian Journal of Zoology. 38: 345-356.

Watson, R. A. Metazoan parasites from whitefish, cisco, and pike from Southern
Indian Lake, Manitoba. A pre-impoundment and diversion analysis. M. Sc. Thesis. Department of Zoology, University of Manitoba, Winnipeg, Manitoba. 197 p.

Watson, R. A. and Dick, T. A. 1979. Metazoan parasites of whitefish Coregonus clupeaformis (Mitchill) and C. artedii Lesueur from Southern Indian Lake, Manitoba. Journal of Fish Biology. 15: 579-587.

Watson, R. A. and Dick, T. A. 1980. Metazoan parasites of pike, Esox lucius Linnaeus, from Southern Indian Lake, Manitoba. Journal of Fish Biology. 17: 255261.

Watson, R. G. 1981. Metorchis conjunctus (Cobbold, 1860) Looss, 1899 (Trematoda: Opisthorchiidae): isolation of metacercariae from fish hosts. Canadian Journal of Zoology. 59: 2010-2013.

Welch, H. E. 1950a. Appendix No. 6. Triaenophorus investigations in the Thunder Bay district, Ontario, p. 22-23. In: K H. Doan. Fisheries Research Board of Canada. Annual Report of the Central Fisheries Research Station for 1950, with Investigators' Summaries as Appendices.

Welch, H. E. 1950b. Triaenophorus investigations in the Thunder Bay district. Fisheries Research Board of Canada. Manuscript Report (Biology). No. 522: 13 p.

Welch, H. E. 1951. Appendix No. 8. Triaenophorus investigations in the Thunder Bay district, Ontario, in 1951, p. 23-26. In: K. H. Doan. Fisheries Research Board of Canada. Annual Report of the Central Fisheries Research Station for 1951, with Investigators' 
Summaries as Appendices.

Welch, H. E. 1952a. Factors affecting the infection of whitefish, Coregonus clupeaformis (Mitchill), by the tapeworm, Triaenophorus crassus Forel, in the Thunder Bay district of Ontario. M. A. Thesis. Department of Zoology, University of Toronto, Toronto, Ontario.

Welch, H. E. 1952b. Factors affecting the infection of whitefish, Coregonus clupeaformis (Mitchill), by the tapeworm, Triaenophorus crassus Forel, in the Thunder Bay district of Ontario. Fisheries Research Board of Canada. Manuscript Report (Biology). No. 523: 87 p.

Wells, P. R. and Cone, D.K. 1990. Experimental studies on the effect of Gyrodactylus colemanensis and G. salmonis (Monogenea) on density of mucous cells in the epidermis of fry of Oncorhynchus mykiss. Journal of Fish Biology. 37: 599-603.

Wheaton, R. R. 1952. Appendix No. 30. An ecological and taxonomic study of the "blackwhitefish" in Great Slave Lake, p. 69-70. In: W. A. Kennedy. Fisheries Research Board of Canada. Annual Report of the Central Fisheries Research Station for 1952, with Investigators' Summaries as Appendices.

Wheaton, R. R. and Hazen, G. E. M. 1951. Whitefish-Triaenophorus crassus investigations on Nesslin Lake, Saskatchewan, 1950. Fisheries Research Board of Canada. Manuscript Report (Biology). No. 494: 15 p.

Wheaton, R. R. and Rawson, D. S. 1949a. Appendix No. 2. Triaenophorus investigations in Nesslin Lake, Saskatchewan, p. 16-18. In:
K. H. Doan. Fisheries Research Board of Canada. Annual Report of the Central Fisheries Research Station for 1949, with Investigators' Summaries as Appendices.

Wheaton, R. R. \& Rawson, D. S. 1949b. Appendix No. 4. Triaenophorus investigations on Mosher Lake, Saskatchewan, p. 20-26. In: K. H. Doan. Fisheries Research Board of Canada. Annual Report of the Central Fisheries Research Station for 1949, with Investigators' Summaries as Appendices. White, F. M. 1940. Studies on the morphology of a new species of Cystidicola (Nematoda: Thelaziidae) from the swim bladder of the lake trout. Journal of Parasitology. 26(Supplement): 39-40.

White, F. M. 1940. Studies on the morphology of Cystidicola cristivomeri sp. nov. (Nematoda: Thelaziidae) from the swim bladder of the lake trout. Proceedings of the Indiana Academy of Sciences. 50: 211.

White, F. M. and Cable, R. M. 1942. Studies on the morphology of Cystidicola cristivomeri sp. nov. (Nematoda: Thelaziidae) from the swim bladder of the lake trout, Cristivomer namaycush (Walbaum). American Midland Naturalist. 28: 416-423.

Wiles, M. 1975. The glochidia of certain Unionidae (Mollusca) in Nova Scotia and their fish hosts. Canadian Journal of Zoology. 53: 33-41.

Wilson, C. B. 1916. Copepod parasites of freshwater fishes and their economic relations to mussel glochidia. Bulletin of the United States Bureau of Fisheries. 34: 331-374.

Wilson, C. B. 1920. Report on the parasitic 
Copepoda collected during the Canadian Arctic Expedition, 1913-1918. Report of the Canadian Arctic Expedition, 1913-1918. 7(Part1): 1-16.

Wilson, C. B. 1936. Argulus canadensis from Cape Breton Island. Journal of the Biological Board of Canada. 45: 1083-1092.

Wilson, K. A. and Ronald, K. 1967. Parasite fauna of the sea lamprey (Petromyzon marinus von Linné) in the Great Lakes region. Canadian Journal of Zoology. 45: 1083-1092.

Wobeser, G., Kratt, L. F., Smith, R. J., and Acompandao, G. 1976. Proliferative branchitis due to Tetraonchus rauschi (Trematoda: Monogenea) in captive Arctic grayling (Thymallus arcticus). Journal of the Fisheries Research Board of Canada. 33: 1817-1821.

Wong, P. L. and Anderson, R. C. 1982. The transmission and development of Cosmocephalus obvelatus (Nematoda: Acuarioidea) of gulls (Laridae). Canadian Journal of Zoology. 60: 1426-1440.

Wood, R. A. and Mizelle, J. D. 1957. Studies on monogenetic trematodes. XXI. North American Gyrodactylidae, Dactylogyrinae and a new host record for Urocleidus dispar (Mueller, 1936). American Midland Naturalist. 57: 183-202.

Woodhead, A. E. 1930. Life history studies on the trematode family Bucephalidae, No. II. Transactions of the American Microscopical Society. 49: 1-17.

Worley, D. E. and Bangham, R. V. 1952. Some parasites of fishes of the upper Gatineau River valley. Ohio Journal of Science. 52: 210-
212.

Wright, B. J., Albert, E. and Curtis, M. A. 1989. The effect of population depletion on the growth, feeding behaviour and parasite community of brook trout (Salvelinus fontinalis) in a subarctic lake. Program \& Abstracts. $28^{\text {th }}$ Annual Meeting, Canadian Society of Zoologists. Bulletin of the Canadian Society of Zoologists. 20(2): 63.

Wright, K. A. and Dechtiar, A. O. 1974. Light and scanning electron microscopy of attachment organs of three monogeneans (Monugenea: Polyopisthocotylea). Canadian Journal of Zoology. 52: 183-187.

Wright, R. R. Contributions to American helminthology. Proceedings of the Canadian Institute, New Series. 1: 54-75.

Wright, R. R. 1882. Notes on American parasitic Copepoda. No. 1. Proceedings of the Canadian Institute, New Series. 1: 243-254.

Zelmer, D. A. 1994. Parasites of yellow perch (Perca flavescens) in Alberta. M. Sc. Thesis. Department of Biological Sciences, University of Calgary, Calgary, Alberta. 103 p.

Zelmer, D. A. and Arai, H. P. 1998. The contributions of host age and size to the aggregated distribution of parasites in yellow perch, Perca flavescens, from Garner Lake, Alberta. Journal of Parasitology. 84: 24-28. 

National Library of Canada

Bibliotheque nationale du Canada

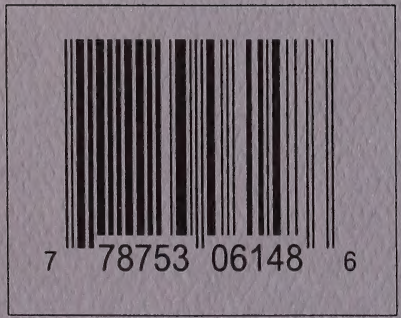

AGRICULTURE, FOOD AND
RURAL DEVELOPMENT

Printed in Canada 